\title{
Study of the thermal diffusion behavior of simple binary mixtures
}

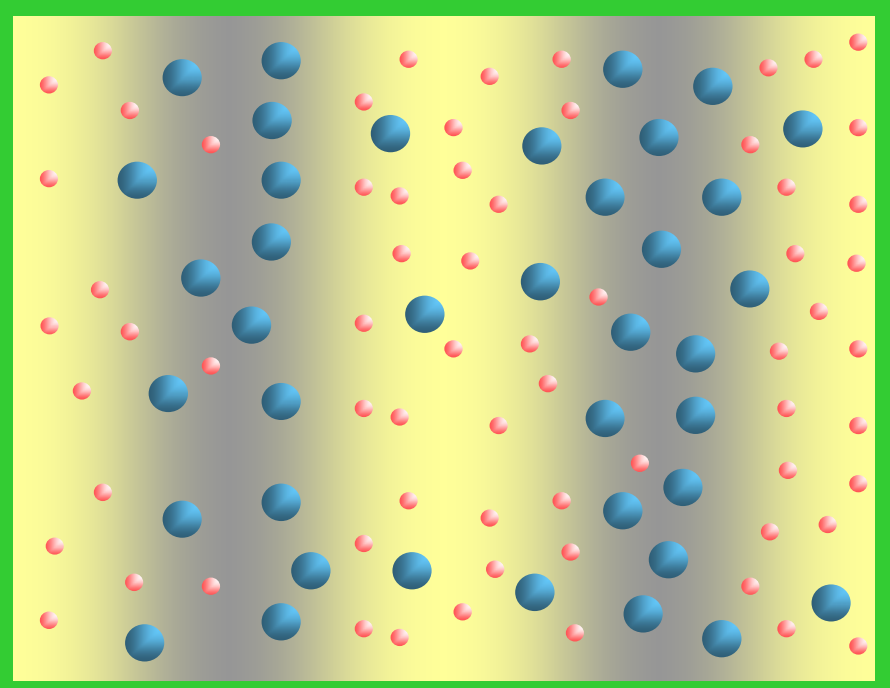

Pavel Polyakov 
STUDY OF THE THERMAL DIFFUSION BEHAVIOR OF SIMPLE BINARY MIXTURES

Pavel Polyakov 
Polyakov, Pavel

Study of the thermal diffusion behavior of simple binary mixtures

Thesis, University of Twente, Enschede, the Netherlands

ISBN: 978-90-365-2754-5

Printed by Grafische Medien, Forschungszentrum Jülich GmbH, Germany

Copyright(C)2008 by P. Polyakov, Weiche Materie, Institut für Festkörperforschung,

Forschungszentrum Jülich GmbH, Germany

Front cover: Sketch of the thermophoretic motion of a binary mixture in a temperature grating

Typeset in $\mathrm{LT}_{\mathrm{E}} \mathrm{X}$, by the author 
STUDY OF THE THERMAL DIFFUSION BEHAVIOR OF SIMPLE BINARY MIXTURES

\author{
DISSERTATION
}

to obtain

the doctor's degree at the University of Twente,

on the authority of the rector magnificus,

prof. dr. W.H.M. Zijm,

on account of the decision of the graduation committee,

to be publicly defended

on Thursday, 20 November 2008 at 13:15

by

\title{
Pavel Polyakov
}

born on 13 May 1982

in Novosibirsk, USSR 
This dissertation has been approved by:

the promoters

Prof. dr. W. J. Briels

Prof. dr. J. K. G. Dhont

and the assistant-promoter

Dr. S. Wiegand 


\section{Contents}

1 Introduction $\quad 1$

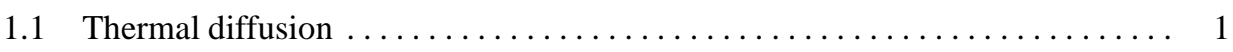

1.2 Thermodynamic of irreversible processes $\ldots \ldots \ldots \ldots \ldots \ldots \ldots \ldots \ldots$

1.2.1 Entropy production due to the heat transport $\ldots \ldots \ldots \ldots \ldots \ldots$

1.2.2 Entropy production due to the mass transport $\ldots \ldots \ldots \ldots \ldots \ldots \ldots$

1.2.3 The coupling between mass and heat transport $\ldots \ldots \ldots \ldots \ldots \ldots$

1.3 Theoretical description of the Soret effect $\ldots \ldots \ldots \ldots \ldots \ldots \ldots \ldots \ldots$

1.3.1 Theoretical approaches $\ldots \ldots \ldots \ldots \ldots \ldots \ldots \ldots \ldots \ldots \ldots \ldots \ldots \ldots \ldots \ldots \ldots$

1.3.2 Comparison with experiment $\ldots \ldots \ldots \ldots \ldots \ldots \ldots \ldots \ldots \ldots \ldots \ldots \ldots \ldots \ldots$

1.4 Calculation of the Soret coefficient $\ldots \ldots \ldots \ldots \ldots \ldots \ldots \ldots \ldots \ldots \ldots$

1.4.1 Two-chamber lattice model $\ldots \ldots \ldots \ldots \ldots \ldots \ldots \ldots \ldots \ldots \ldots$

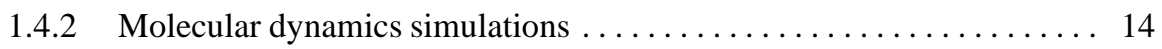

1.5 Experimental methods $\ldots \ldots \ldots \ldots \ldots \ldots \ldots \ldots \ldots \ldots \ldots \ldots \ldots \ldots \ldots \ldots \ldots$

1.5.1 Thermal Diffusion Forced Rayleigh Scattering ............. 18

1.5.2 Thermal lens method .......................... 23

1.5.3 Thermogravitational column $\ldots \ldots \ldots \ldots \ldots \ldots \ldots \ldots \ldots \ldots \ldots \ldots \ldots \ldots \ldots$

1.5.4 Thermal diffusion cells ....................... 28

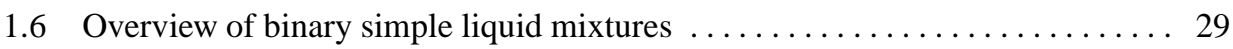

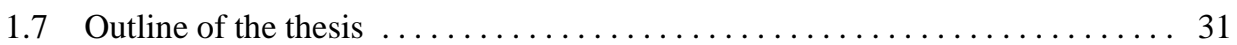

2 Mixtures of spherical molecules $\quad 35$

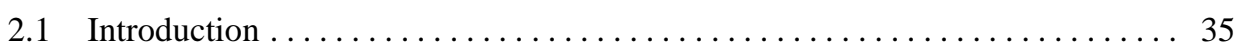

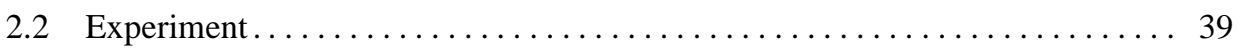

2.2.1 Sample Preparation........................... 39

2.2.2 Refractive index increment measurements............... 40

2.2.3 TDFRS experiment and data analysis $\ldots \ldots \ldots \ldots \ldots \ldots \ldots \ldots \ldots$

2.3 Results $\ldots \ldots \ldots \ldots \ldots \ldots \ldots \ldots \ldots \ldots \ldots \ldots \ldots \ldots \ldots \ldots \ldots \ldots \ldots \ldots$ 


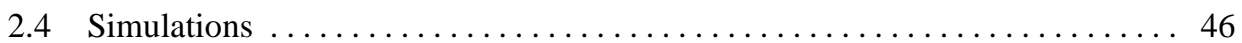

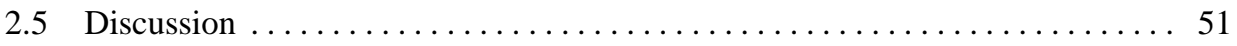

2.5.1 Comparison of the experimental and simulation results $\ldots \ldots \ldots \ldots 51$

2.5.2 Contributions to the Soret coefficient. ............... 53

2.5.3 Discussion of the effect of the moment of inertia. ........... 54

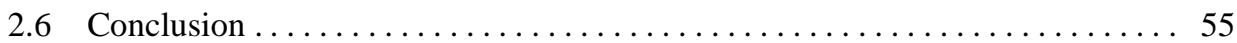

3 Mixtures of linear alkanes $\quad 57$

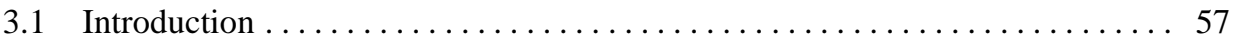

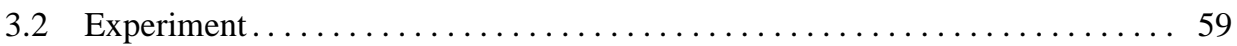

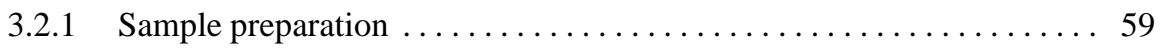

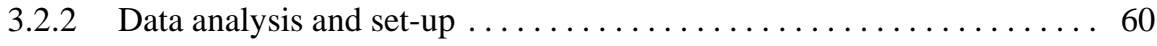

3.2.3 Density measurements ......................... 64

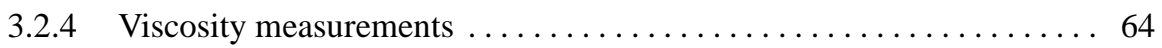

3.2.5 Refractive index increments ...................... 64

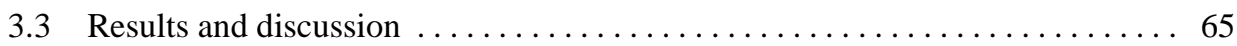

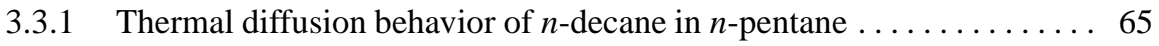

3.3.2 Thermal diffusion behavior of $n$-decane in various alkane at equal

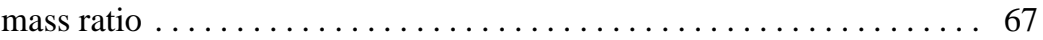

3.4 Conclusion $\ldots \ldots \ldots \ldots \ldots \ldots \ldots \ldots \ldots \ldots \ldots \ldots \ldots \ldots \ldots \ldots \ldots \ldots \ldots$

4 Mixtures of alakane in benzene: experiment and lattice calculations 73

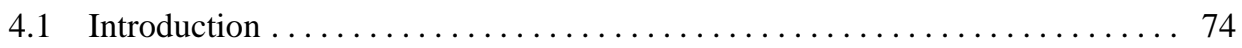

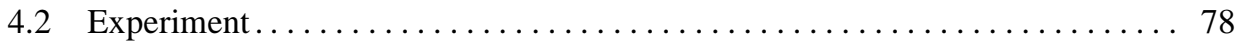

4.2.1 Sample preparation $\ldots \ldots \ldots \ldots \ldots \ldots \ldots \ldots \ldots \ldots \ldots \ldots \ldots$

4.2.2 Refractive index increment measurements .............. 79

4.2.3 TDFRS experiment and data analysis ................. 79

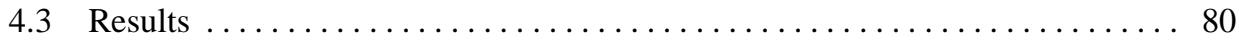

4.4 Lattice model for the Soret effect in alkane/ benzene mixtures . . . . . . . . . 85

4.4.1 Calculation of Soret coefficients $\ldots \ldots \ldots \ldots \ldots \ldots \ldots \ldots$

4.4.2 Comparison with experimental data $\ldots \ldots \ldots \ldots \ldots \ldots \ldots$

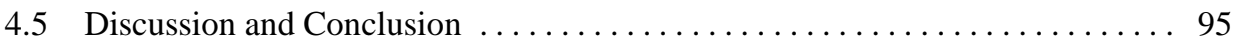


4.6 Appendix: Determination of system-dependent parameters for alkane/benzene

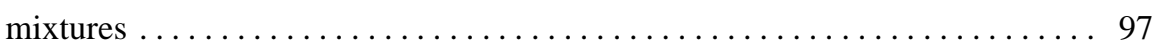

5 Mixtures of branched heptane in benzene: RNEMD simulations 101

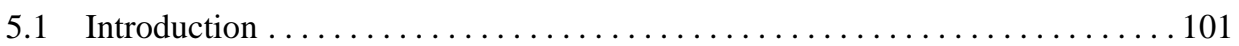

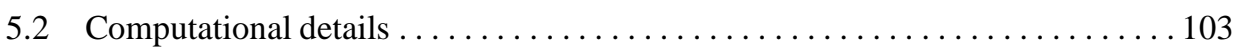

5.3 Equilibrium molecular dynamics simulations $\ldots \ldots \ldots \ldots \ldots \ldots \ldots \ldots \ldots$

5.4 Non equilibrium molecular dynamics simulations $\ldots \ldots \ldots \ldots \ldots \ldots \ldots \ldots$

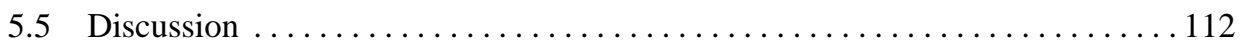

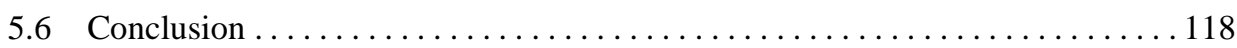

6 Mixtures of associated molecules $\quad 119$

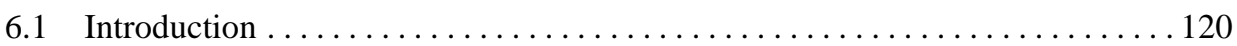

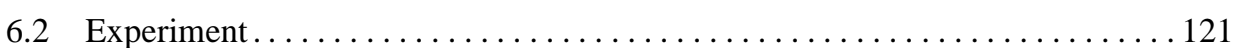

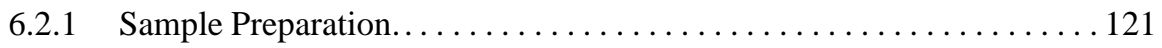

6.2.2 Refractive index increment measurements................ 122

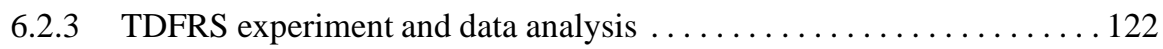

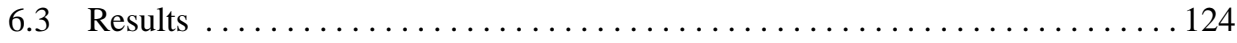

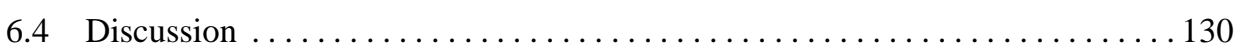

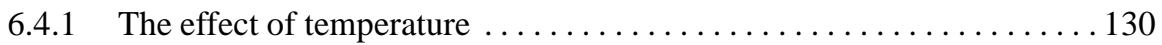

6.4.2 Relation between the thermal diffusion motion and a structural change

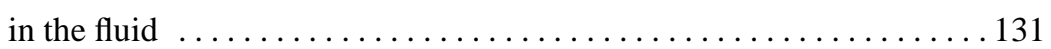

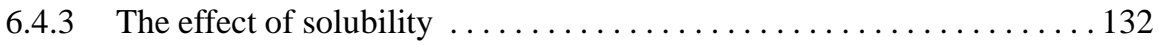

6.4.4 The effect of hydrophilic interactions $\ldots \ldots \ldots \ldots \ldots \ldots \ldots \ldots \ldots \ldots \ldots$

6.4.5 Effects in alcoholic mixtures with DMSO ............... 133

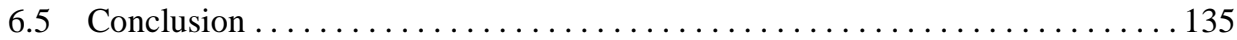

7 Investigation of the Soret effect by the thermal lens technique 137

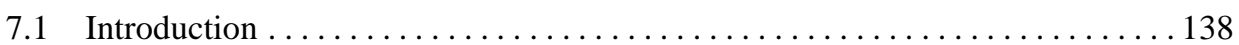

7.2 Experiment and working equations $\ldots \ldots \ldots \ldots \ldots \ldots \ldots \ldots \ldots \ldots \ldots \ldots \ldots \ldots \ldots \ldots \ldots$

7.2.1 Sample Preparation............................ 141

7.2.2 Refractive index increment measurements................ 142 
7.2.3 TDFRS experiment and data analysis $\ldots \ldots \ldots \ldots \ldots \ldots \ldots \ldots . \ldots \ldots 2$

7.2.4 Thermal lens experiment and data analysis ............... 144

7.3 Results and Discussion . . . . . . . . . . . . . . . . . . 150

7.3.1 Nonionic surfactant $\mathrm{C}_{12} \mathrm{E}_{6}$ in water $\ldots \ldots \ldots \ldots \ldots \ldots \ldots \ldots \ldots \ldots \ldots \ldots \ldots \ldots \ldots$

7.3.2 DMSO in water ............................... 153

7.3.3 Ionic liquid EMIES in butanol . . . . . . . . . . . . . . . 154

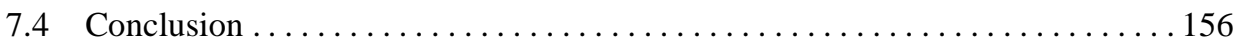

$\begin{array}{lr}\text { Summary } & 157\end{array}$

$\begin{array}{ll}\text { Acknowledgment } & 167\end{array}$

$\begin{array}{lr}\text { Curriculum Vitae } & 169\end{array}$

$\begin{array}{ll}\text { Publications } & 171\end{array}$

$\begin{array}{ll}\text { Bibliography } & 173\end{array}$ 


\section{Introduction}

\subsection{Thermal diffusion}

The mass transport of chemical species in response to a temperature gradient, referred to the Soret effect or thermal diffusion, leads under certain conditions to a separation of the chemical constituents.

According to the phenomenological equations of irreversible thermodynamics, thermal diffusion in a binary fluid mixture is described by the flux $J$ of one of the components in response to a temperature $\nabla T$ and concentration gradient $\nabla c$ [35].

$$
\mathbf{J}=-\rho D \nabla c-\rho D_{T} c(1-c) \nabla T
$$

where $c$ is the mass fraction of the first component, $\rho$ is the density of the mixture, $D$ is the mutual diffusion coefficient, and $D_{\mathrm{T}}$ is the thermal diffusion coefficient. In the steady state $(J=0)$ the concentration gradient is characterized by the Soret coefficient $S_{\mathrm{T}}=D_{\mathrm{T}} / D$. The positive Soret coefficient of the component with the weight fraction $c$ implies that this component moves to the cold region.

This effect was first observed by Ludwig more than 150 years ago [89]. Soret performed the first systematic investigations [157]. In his experiments, a tube with a length of $30 \mathrm{~cm}$ with aqueous $\mathrm{NaCl}$ or $\mathrm{KNO}_{3}$ solution was heated from the one end $\left(78^{\circ} \mathrm{C}\right)$ and cooled from the other one $\left(\sim 18{ }^{\circ} \mathrm{C}\right)$. After approximately 17 days Soret found that the salt concentrated in the cold region. In his second paper [158] he noted the importance of the waiting time. For other two $\mathrm{KCl}$ and $\mathrm{LiCl}$ aqueous solutions he waited already for 50 days in order to reach equilibrium state. Another important finding is that for various aqueous salt solutions with the same initial composition the difference in concentration between the cold and the warm ends 
of the tube was an increasing function of the molecular weight. This result is in agreement with the "rule of thumb" that the heavier component often moves to the cold side.

The Soret effect plays a crucial role in many naturally occurring processes such as thermohaline convection in oceans [24], component segregation in solidifying metallic alloys [192] or volcanic lava $[31,175]$. The technical applications are isotope separation of liquids and gaseous mixtures [122], thermal field flow fractionation of polymers and colloids [145, 29], identification and separation of crude oil components [30], coating of metallic items, etc. This phenomenon is supposed to play an important role in crystal growth [136] and combustion [128]. Precise values of the Soret coefficient are needed in the analysis of the phenomena of hydrodynamic instability [169], testing the theory of non-equilibrium fluctuations in liquid mixtures [149], and for the design of a separation setup [87].

In spite of discovering this effect more than 150 ago and its wide technical applications there is so far only a limited microscopic understanding of the thermal diffusion processes in liquids. The precise prediction of $S_{\mathrm{T}}$ from theory and simulations and even the experimental determination for more complex systems is often a challenge.

Many experimental techniques have been developed for investigation of the Soret effect: thermogravitational columns [20], thermal lens [115], diffusion cells [188, 121], thermal diffusion forced Rayleigh scattering technique [76, 181], thermal field flow fractination [145] and microfluidic fluorescence [43]. However, only in the last decade it became possible to get reproducible values of $S_{\mathrm{T}}$ with different methods for toluene/hexane mixture [188, 75] and for binary mixtures of 1,2,3,4-tetrahydronaphthalene, $n$-dodecane and isobutylbenzene [116]. The reasons for the previous deviations are manifold. On one hand technical imperfectnesses are responsible and on the other hand the experiments are sometimes disturbed by convection effects. Experimental measurements of $S_{\mathrm{T}}$ in polymer solutions [146], micellar solutions [109, 114], colloidal dispersions [114, 106], magnetic fluids [100] and bio-macromolecules [44] are even more complicated due to the longer measurement time, incomplete mixing and polydispersity. Typically, the Soret coefficient $S_{\mathrm{T}}$ in simple liquid mixtures lies in the range $10^{-5}<S_{\mathrm{T}}<10^{-3} \mathrm{~K}^{-1}[179,111]$, while for slower diffusion mixtures such as polymer solutions, micellar solutions and colloidal dispersions $S_{\mathrm{T}}$ is in range $10^{-2}<S_{\mathrm{T}}<10^{2} \mathrm{~K}^{-1}$ $[74,72,109,67,44,106]$.

With the development of new simulation techniques reasonable predictions of Soret co- 
efficients for low molecular weight mixtures became possible [64, 104, 113, 190]. The problems with theoretical description of these systems are caused by the high sensitivity of the thermal diffusion factors to the values of the partial molar properties [11]. The present equations of state require modification for the precise calculation of the Soret coefficient. At the same time, the theoretical concepts developed for colloidal dispersions [171, 115, 106, 150, 50] show a better agreement with experiments. A detailed overview can be found in $\mathrm{PhD}$ thesis by Ning [105].

In this thesis we focus on the investigation of thermal diffusion behavior in simple liquid mixtures experimentally and by simulations. Experiments were performed with the thermal diffusion forced Rayleigh scattering setup and the thermal lens method. Simulation were performed with the so-called YASP (Yet Another Simulation Package) work package, developed by Müller-Plathe.

\subsection{Thermodynamic of irreversible processes}

In this section we present the description of the Soret effect in terms of general fluxes and forces. First, we derive the equation for entropy production due to the heat and mass transports. Secondly, we describe the coupling between these two processes - so called Soret effect.

\subsubsection{Entropy production due to the heat transport}

The second principle of thermodynamics postulates the existence of a function of state, called entropy. Now we consider a system consisting of two subsystems, maintained at the temperatures of $T_{1}$ and $T_{2}$, respectively (c.f. Fig. 1.1A). The entropy of the system is an extensive property, therefore:

$$
d S=d S_{1}+d S_{2}
$$

Considering the classic definition of entropy we end up with

$$
d S=\frac{d_{e}^{1} Q}{T_{1}}+\frac{d_{e}^{2} Q}{T_{2}}+d_{i} Q\left(\frac{1}{T_{1}}-\frac{1}{T_{2}}\right),
$$




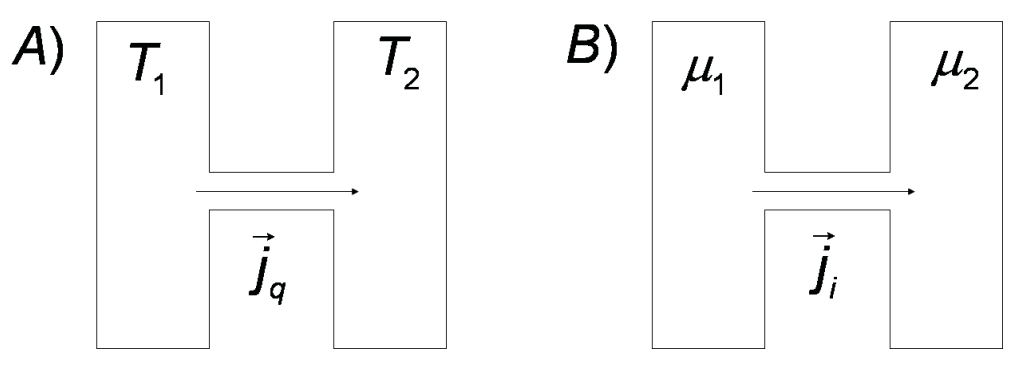

Figure 1.1: The heat (A) and mass (B) transport in liquid.

where $d_{i} Q$ is the heat received by subsystem one from subsystem two and $d_{e}^{1} Q$ (or $d_{e}^{2} Q$ ) is the heat supplied to subsystem one (or two) from the outside. The first part of the entropy change:

$$
d_{e} S=\frac{d_{e}^{1} Q}{T_{1}}+\frac{d_{e}^{2} Q}{T_{2}}
$$

is due to the exchange of heat with exterior, while the second part

$$
d_{i} S=d_{i} Q\left(\frac{1}{T_{1}}-\frac{1}{T_{2}}\right)
$$

results from the irreversible heat flow inside the system. Here, we can postulate, that the entropy increase $d_{i} S$, due to the change inside the system, is never negative. In our case it means that positive heat can not be transferred from the colder subsystem to the warmer one

$$
d_{i} Q>0 \text { when } \frac{1}{T_{1}}-\frac{1}{T_{2}}>0, \quad d_{i} Q<0 \text { when } \frac{1}{T_{1}}-\frac{1}{T_{2}}<0 .
$$

Furthermore, we shall make use of the entropy production per unit time

$$
\frac{d_{i} S}{d t}=\frac{d_{i} Q}{d t}\left(\frac{1}{T_{1}}-\frac{1}{T_{2}}\right)>0
$$

The sources of the entropy grow are on the border between subsystems, where the temperature jump happens (c.f. Fig. 1.1A). In general case the entropy production is proportional to $\nabla \frac{1}{T}$ and equal to zero when thermal equilibrium is established $\left(T_{1}=T_{2}\right)$. The derivative $d_{i} Q / d t$ can be associated with the heat flux $\vec{j}_{q}$, caused by the difference in temperature. 


\subsubsection{Entropy production due to the mass transport}

Mass transport or diffusion can also lead to an increase of the entropy. In order to derive similar expression for the enthalpy production in the presence of diffusion we will start with the Gibbs equation

$$
T \Delta s_{V}=\Delta u_{V}-\sum \mu_{i} \Delta \theta_{i}
$$

where $s_{V}$ and $u_{V}$ are entropy and internal energy normalized to the volume of the system, $\theta_{i}$ is the volume fraction and $\mu_{i}$ is the chemical potential of the $i$ component. In order to make the calculations simpler we assume a constant temperature and pressure in system. Under these conditions

$$
\frac{\partial u_{V}}{\partial T}=0
$$

and velocity vector $(\vec{v})$ is equal to zero in the equation for dynamic derivative

$$
\frac{d}{d T}=\frac{\partial}{\partial T}+\vec{v} \nabla
$$

The concentration $\theta_{i}$ inside the volume element can only change because of the flow $\overrightarrow{j_{i}}$ of the fluid across its boundary

$$
\frac{d \theta_{i}}{d t}=-\nabla \cdot \vec{j}_{i}
$$

Combining Eq. 1.8, 1.9 and 1.11 is recovered in the following form

$$
\frac{d s_{V}}{d t}=\nabla \cdot\left(\sum \frac{\mu_{i} \vec{j}_{i}}{T}\right)-\sum \overrightarrow{j_{i}} \cdot \frac{\nabla \mu_{i}}{T} .
$$

The first term in Eq. 1.12 is the divergence of the flux, while the second one is the entropy source. The diffusion flux is caused then by the difference in the chemical potentials (c.f. Fig. 1.1B).

\subsubsection{The coupling between mass and heat transport}

The general expression for entropy production due to the heat and mass transport can be written as a sum of the product of generalized forces $\vec{F}_{k}$ and the corresponding flows $\vec{j}_{k}$ (c.f. Sec. 1.2.1 and 1.2.2)

$$
\frac{d s_{V}}{d t}=\vec{j}_{q} \cdot \nabla\left(\frac{1}{T}\right)-\sum \vec{j}_{i} \cdot \frac{\nabla \mu_{i}}{T}=\sum \vec{j}_{k} \cdot \vec{F}_{k} .
$$


For a small deviation in the forces from their equilibrium value of zero, the flows can be expected to be linear functions of the forces. Accordingly, the following relation between the flows and the forces is assumed

$$
\vec{j}_{k}=\sum L_{k i} \vec{F}_{i}
$$

Here it is important to note, that a force such as $F_{i}$ causes not only the flow of the component $i$ but also the flow of component $k$ (so called cross effect). The coupling between heat and mass flow produces, basically, two effects the Soret and the Dufor effects. In the Soret effect the mass flow is driven by the heat flow. In the Dufor effect, the heat flow is driven by concentration gradient. Using Gibbs-Duhem relation and assuming zero flow

$$
n_{1} \nabla \mu_{1}+n_{2} \nabla \mu_{2}=0, \quad \vec{j}_{1} v_{1}+\vec{j}_{2} v_{2}=0
$$

where $v_{k}$ is partition molar volume and $n_{k}$ is number density (particles per cubic centimeter), Eq. 1.13 can be rewritten in a simpler form [79]

$$
\sigma=\vec{j}_{q} \cdot \nabla\left(\frac{1}{T}\right)-\frac{1}{T}\left(1+\frac{v_{1} n_{1}}{v_{2} n_{2}}\right)\left(\nabla \mu_{i}\right)_{p, T} \cdot \vec{j}_{1} .
$$

The equation for mass flux in the form of Fourier law can then be obtained by considering $\nabla \mu_{1}=\left(\partial \mu_{1} / \partial n_{1}\right) \nabla n_{1}$ and $\nabla(1 / T)=-\left(1 / T^{2}\right) \nabla T$

$$
\vec{j}_{1}=L_{1 q} \nabla\left(\frac{1}{T}\right) \nabla T-L_{q 1} \frac{1}{T}\left(1+\frac{v_{1} n_{1}}{v_{2} n_{2}}\right)\left(\frac{\partial \mu_{i}}{\partial n_{1}}\right) \nabla n_{1} .
$$

This equation is usually written in another form

$$
\vec{j}_{1}=\rho D \nabla c-\rho c(1-c) D_{T} \nabla T
$$

where $\rho$ is the density of the mixture, $c$ is the mass fraction of the component $1, D$ and $D_{\mathrm{T}}$ are the mutual diffusion and thermal diffusion coefficients, respectively. In the case of the steady state $j_{1}=0$ and the Soret coefficient is given by

$$
S_{\mathrm{T}}=\frac{D_{T}}{D}=\frac{1}{c(1-c)} \frac{\nabla c}{\nabla T},
$$

The sign of the Soret coefficient determines the direction of thermal diffusive motion (a positive $S_{\mathrm{T}}$ of the component 1 corresponds to the component 1 moving to the colder regions of the fluid $[52,181])$. Other two combinations of the mutual and thermal diffusion coefficients can be also found in literature: the thermal diffusion factor $\alpha_{\mathrm{T}}=S_{\mathrm{T}} T$ and the thermal diffusion ratio $k_{\mathrm{T}}=S_{\mathrm{T}} T c_{1}\left(1-c_{1}\right)$. 


\subsection{Theoretical description of the Soret effect}

\subsubsection{Theoretical approaches}

Denbigh et al. [39] had extended the general thermodynamic theory of the Soret effect (c.f. sec.1.2). He considered the system consisting of two subsystem with slightly different temperatures (c.f. Fig. 1.1A). The numbers of particles $N_{i}$ of substance $i$ moving from on subsystem to another due to the Soret effect can be calculated [153]

$$
N_{i}^{I \leftrightarrow I I}=N_{i}^{0} \exp \left(-\frac{Q_{i}^{\star}}{R(T \pm 0.5 \triangle T)}\right),
$$

where $N_{i}^{0}$ is the number of particles of the component $i$ at $t=0, Q_{i}^{\star}$ is the heat of transport (the heat energy required to activate the diffusion process, or the net heat energy which must be absorbed at the lattice site from which a particle moves out and has been replaced by some incoming particles in order to maintain a constant local temperature). From Eq. 1.20

$$
\left(\frac{1}{N_{i}}\right) \frac{\partial N_{i}}{\partial T}=-\frac{Q_{i}^{\star}}{R T^{2}}
$$

In general case (non ideal solutions) the left side of Eq. 1.21 needs to be rewritten in terms of the activity coefficients $a_{i}$

$$
\left(\frac{1}{a_{i}}\right) \frac{\partial a_{i}}{\partial T}=\left(\frac{1}{x_{i}}\right) \frac{\partial \ln a_{i}}{\partial \ln x_{i}} \frac{\partial x_{i}}{\partial T}=\frac{1}{R T} \frac{\partial \mu_{i}}{\partial x_{i}} \frac{\partial x_{i}}{\partial T},
$$

where $x_{i}$ and $\mu_{i}$ are concentration and chemical potential of the component $i$. Thus, the connection between concentration and temperature gradients can be written as

$$
\left(\frac{\partial \mu_{i}}{\partial x_{i}}\right)_{p T} \frac{\partial x_{i}}{\partial T}=-\frac{Q_{i}^{\star}}{T}
$$

For an ideal mixture of two component $\left(\mu_{i}=\mu_{i}^{\star}+R T \ln x_{i}\right)$ the thermal diffusion ratio can be calculated as

$$
\alpha_{T}=-\frac{\partial \ln \left(x_{1} / x_{2}\right)}{\partial T}=\frac{Q_{2}^{\star}-Q_{1}^{\star}}{R T},
$$

After the work of Denbigh, several approaches have been developed to describe the heats of transport.

Rutherford and Drickamer model The mixture was considered to be a random distribution of molecules of approximately the same size and shape [141]. Transport was represented 
as a process of filling and emptying the "holes" between the molecules. The relative probability of the hole left being filled by a molecule of type one or two was related to the ratio of molar fraction of components $\left(x_{1} / x_{2}\right)$. The heats of filling and leaving a hole were expressed in terms of the partial molar enthalpies. The thermal diffusion factor was found to be

$$
\alpha_{T}=-\frac{\left(x_{1} h_{1}^{0.5}+x_{2} h_{2}^{0.5}\right)\left(h_{2}^{0.5}-h_{1}^{0.5}\right)}{2\left(R T-x_{1} x_{2}\left(h_{2}^{0.5}-h_{1}^{0.5}\right)^{2}\right)}
$$

where $h_{i}$ is partial molar enthalpy of the component $i$. This theory was also extended to be applied to binary mixtures composed of molecules with different sizes and shapes. On the basis of this work Dougherty and Drickamer [41] model was developed latter.

Shieh model In 1969 Shieh [153] proposed a new approach based on the BearmanKirkwood-Fixman theory [13]. The thermal diffusion factor was represented as a function of the partial molar heats of vaporization $E_{1}^{v a p}$, partition molar volumes $v_{i}$ and the derivatives of the chemical potential

$$
\alpha_{T}=-\frac{\left(v_{1} E_{2}^{v a p}-v_{2} E_{1}^{v a p}\right)}{2 V\left(\frac{\partial \mu_{1}}{\partial x_{1}}\right)_{T p}},
$$

This method shows satisfactory agreement with experiment for solutions of $n$-heptane and $n$-hexadecane [153].

Kempers model Kempers model [71] is based on the assumption that the steady state has a maximum number of possible microstates. The partition function of the total system $\mathrm{Z}$ was calculated from the partition functions of the subsystems I and II

$$
Z=z^{I} z^{I I}
$$

The partition functions $z^{I}$ and $z^{I I}$ were calculated from the Helmholtz free energy of the corresponding subsystem

$$
z=\exp \left(-\frac{F}{k T}\right)
$$

The change in concentration due to the Soret effect is calculated from the maximization of the partition function. The result for the thermal diffusion ratio is

$$
\alpha_{T}=\frac{v_{1} h_{2}-v_{2} h_{1}}{\left(v_{1} x_{1}+v_{2} x_{2}\right) x_{1}\left(\frac{\partial \mu_{1}}{\partial x_{1}}\right)_{p T}}
$$


This model was tested for 18 different liquid and gaseous mixtures. The calculated Soret coefficient shows agreement within a factor of 2 . It was also noted in this work, that the closer agreement is not expected due to the high sensitivity of the model to the input parameters from the equation of state.

Shukla and Firoozabadi model The approach of Shukla and Firoozabadi [155] is based on the works of Drickamer [141, 42]. Their modified expression incorporates more accurately the thermodynamic properties of a mixture expressed by means of the Peng-Robinson equation of state. The non-equilibrium part in the model is accounted by incorporating the energy of viscous flow. The expression obtained for the thermal diffusion factor in a binary mixture has the form of:

$$
\alpha_{T}=\frac{U_{1} / \tau_{1}-U_{2} / \tau_{2}}{x_{1}\left(\frac{\partial \mu_{1}}{\partial x_{1}}\right)_{T p}}+\frac{\left(V_{2}-V_{1}\right)\left(x_{1} U_{1} / \tau_{1}+x_{2} U_{2} / \tau_{2}\right)}{\left(x_{1} V_{1}+x_{2} V_{2}\right) x_{1}\left(\frac{\partial \mu_{1}}{\partial x_{1}}\right)_{T p}},
$$

where $U_{i}$ and $V_{i}$ are partial molar volume and partial molar internal energy, $\tau_{i}$ is the ratio of the energy of vaporization and the energy of viscous flow for component $i$. This model together with two previously developed models of Kempers [71] and Rutherford [138] were tested for three groups of mixtures: hydrocarbon systems $\mathrm{CH}_{4} / \mathrm{C}_{3} \mathrm{H}_{8}, \mathrm{CH}_{4} / \mathrm{C}_{4} \mathrm{H}_{10}, \mathrm{C}_{7} \mathrm{H}_{16} / \mathrm{C}_{12} \mathrm{H}_{26}$ and $\mathrm{C}_{7} \mathrm{H}_{16} / \mathrm{C}_{16} \mathrm{H}_{34}$; nonpolar nonhydrocarbon systems $\mathrm{Ar} / \mathrm{CO}_{2}, \mathrm{~N}_{2} / \mathrm{CO}_{2}, \mathrm{H}_{2} / \mathrm{N}_{2}$ and $\mathrm{H}_{2} / \mathrm{CO}_{2}$ and hydrocarbon/nonhydrocarbon systems $\mathrm{CH}_{4} / \mathrm{N}_{2}, \mathrm{CH}_{4} / \mathrm{CO}_{2}$. The comparison of theoretical results with experimental data show the better performance of Shukla and Firoozabadi model in comparison with two other models. Kempers model becomes more reliable in nonhydrocarbon mixtures. In contrast the Rutherford model was found to be more reliable than Kempers model in hydrocarbon mixtures but it fails in nonhydrocarbon mixtures.

Semenov model Semenov and Schimpf [151], derived expressions for the thermal diffusion coefficient for a liquid mixture by hydrodynamic approach. They supposed that the local pressure distribution in a stationary state is not uniform due to asymmetry in the distribution of molecules around the particle. The same asymmetry produces also a local volume force on the particle. According to the Navier-Stockes equation

$$
\eta \triangle u=-\nabla \Pi_{l o c}+f_{l o c}
$$

where $\eta$ is the dynamic viscosity of the liquid, $u$ is the velocity of the liquid, $\Pi_{l o c}$ is the local pressure distribution around the particle, and $f_{l o c}$ is a local volume force in the liquid around 
the particle. It was suggested, that the validity of this approach does not depend on the size of the particle if the size is comparable or larger than one of the solvent molecules.

The Soret was derived as a function of specific molar volumes $v_{i}$, Hamaker constants $A_{i}$, thermal expansion coefficient $\alpha_{T i}$, volume fraction $\phi$

$$
S_{\mathrm{T}}=\frac{\frac{4}{3} \frac{A_{2}}{k T}\left(\sqrt{\frac{A_{1}}{A_{2}}}-1\right)\left[\alpha_{T 1}(1-\phi) \frac{v_{2}}{v_{1}} \sqrt{\frac{A_{1}}{A_{2}}}+\alpha_{T 2} \phi\right]}{\phi+\frac{v_{1}}{v_{2}}(1-\phi)+\frac{4}{3} \frac{A_{2}}{k T}\left(\frac{v_{2}}{v_{1}} \sqrt{\frac{A_{1}}{A_{2}}}-1\right)\left(1-\sqrt{\frac{A_{1}}{A_{2}}}\right)(1-\phi) \phi} .
$$

The model shows satisfactory agreement with experiment for toluene/ $n$-hexane mixture [151]. The application of this model to other mixtures is rather difficult due to the unknown Hamaker constants for most of the common solvents.

\subsubsection{Comparison with experiment}

In Sec. 1.3.1 we have presented several theoretical models for the description of the Soret effect. In some cases theoretical results have been compared with the experimental results. Nevertheless, a more detailed comparison between experimental results and the different models is required in order to get a better feeling for the strength and the weakness of the various models.

Bagnoli [11] have compared the values of the Soret coefficient calculated from different thermodynamic models but not for the hydrodynamic model by Semenov and Schimpf with different sets of experimental data (71 binary mixtures). Several equations of state were used to determine the thermodynamic properties needed for the calculations. It was found, that none of the models describes correctly the thermal diffusion factor for all the mixtures. The Haase model was found to be the best for the systems of $n$-Pentane $+n$-Decane and Methane $+n$-Butane. The mixtures of Benzene + Cyclohexane and Methane $+n$-Propane are well described by Shukla and Firoozabadi model as well as by the Haase model. At the same time the Haase model can not be treated as universal because it was only able to predict the correct sign for 50 mixtures out 71 mixtures. The Kempers approach together with Shukla and Firoozabadi model, estimated the correct sign for 36 mixtures. The Kempers model often overestimates the Soret coefficient with respect to the experimental data. Both models of Dougherty and Drickamer do not provide reasonable description for the investigated mixtures. For most of the mixtures, these models together with Shieh model give the opposite 
sign of the Soret coefficient with respect to Kempers and Shukla and Firoozabadi approaches.

Recently, Jiang et al. [68] compared experimental results for ethanol/water mixtures with predictions of existing theoretical approaches (Haase, Kempers, Dougherty and Drickamer, Shukla and Firoozabadi models). The thermodynamic properties of water/ethanol mixtures were calculated using the Cubic Plus Association equation of state. Dougherty and Drickamers model as well as Kempers and Haase model predict 30 times higher $S_{\mathrm{T}}$ than in the experiment observed. The Soret coefficient predicted by the Firoozabadi model is about 7 times larger than experimental value. At the same time, none of these thermodynamic models can predict the sign change of $S_{\mathrm{T}}$ in the water rich region.

In conclusion none of the thermodynamic models describe the thermal diffusion behavior of all systems well. For particular systems the experimental values agree with the model while for others the predictions fails by an order of magnitude. Additionally, the calculated thermal diffusion factors are very sensitive to the values of the partition molar properties calculated from chosen equations of state. This requires the improvement of the equations of state.

\subsection{Calculation of the Soret coefficient}

In this section we present two simulation approaches for the calculation of the Soret coefficient. The first one, is a simple lattice model, based on statistical mechanics, while the second one, so called heat exchange algorithm, is based on the integration of the Newtonian's equations of motion.

\subsubsection{Two-chamber lattice model}

In the canonical description, the system is described by its microscopic states, which we denote by index $i$. Each state $i$ has an energy $E_{i}$. The canonical partition function is given by

$$
Z=\sum e^{-\beta E_{i}}
$$

where the "inverse temperature" $\beta$ is defined as $\left(k_{B} T\right)^{-1}$. The probability that the system occupies a microstate $i$ is given by

$$
P_{i}=\frac{1}{Z} \sum e^{-\beta E_{i}} .
$$




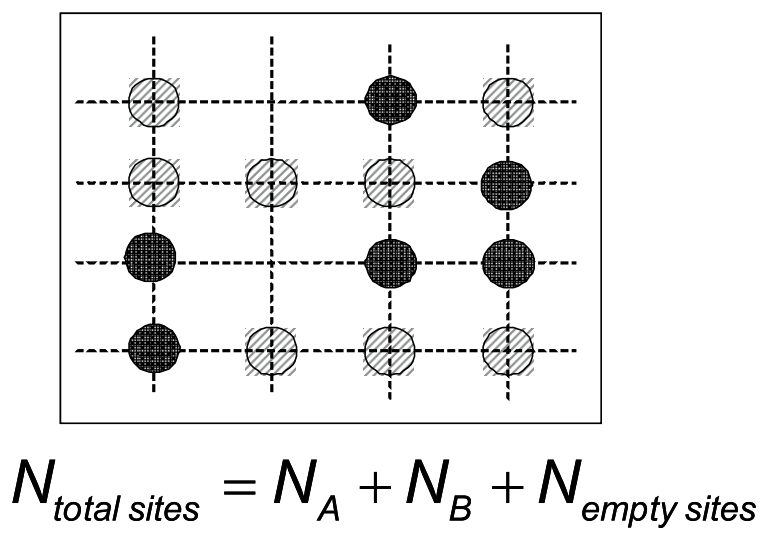

Figure 1.2: Illustration of the lattice model for simulating a binary mixture in equilibrium.

The partition function can be related to thermodynamic properties. For example the ensemble average for the energy $E$ is equal to the sum of the microstate energies $E_{i}$ weighted by their probabilities

$$
E=\sum E_{i} P_{i}=\frac{1}{Z} \sum E_{i} e^{-\beta E_{i}}=-\frac{1}{Z} \frac{\partial}{\partial \beta} Z=-\frac{\partial \ln Z}{\partial \beta} .
$$

In the same way the heat capacity and the pressure can be calculated

$$
C_{v}=\frac{1}{k_{B} T^{2}} \frac{\partial^{2} \ln Z}{\partial \beta^{2}} \quad P=k_{B} T \frac{\partial \ln Z}{\partial V} .
$$

Thus, if we know the partition function we can calculate any thermodynamic property for our system.

This approach can be applied to describe a liquid mixture. In order to calculate the partition function we need to define the microstate, calculate the number of possible microstates and calculate the energy $\left(E_{i}\right)$ of each microstate. The microstate in this case is associated with the given distribution of molecules in the simulation box (c.f. Fig. 1.2). The simulation box is treated as a fixed number of lattice sites ( $\left.N_{\text {totalsites }}\right)$. One given molecule (type A or B) occupies one site (in general case the size of the molecules can also be taken into account). The number of possible microstates $g\left(N_{\text {totalsites }}, N_{A}, N_{B}\right)$ can be determined from combinatoric rules. In the simplest case the energy of different microstates for a given concentration can be assumed to be equal. The partition function of the system can then be written in a 


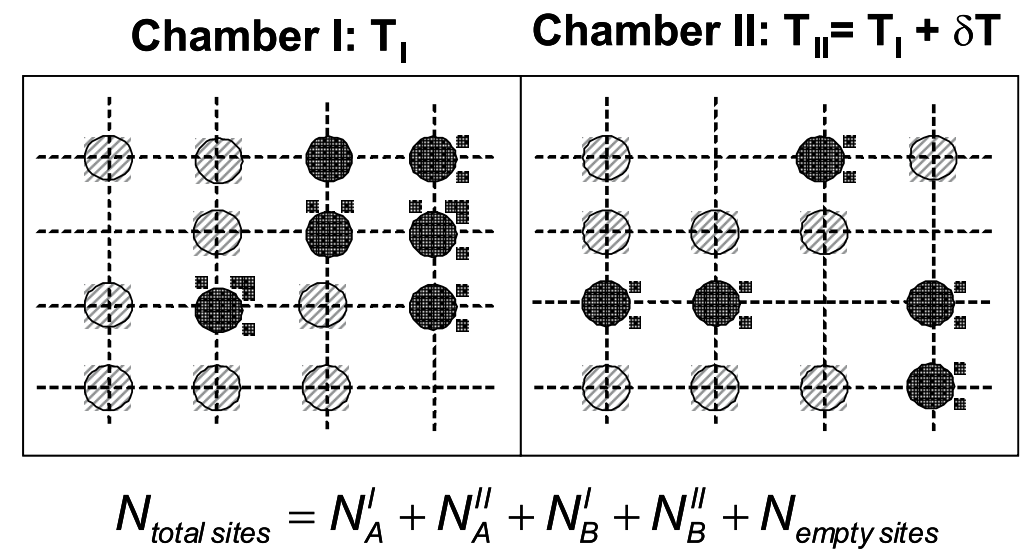

Figure 1.3: Illustration of the lattice model for a simulation of a binary mixture in the stationary state.

simple form

$$
Z\left(N_{\text {total sites }}, N_{A}, N_{B}\right)=g\left(N_{\text {total sites }}, N_{A}, N_{B}\right) e^{-\beta E\left(N_{\text {total sites }}, N_{A}, N_{B}\right)} .
$$

In the simplest case the energy of the given microstate can be written in terms of intermolecular interaction energies $\varepsilon_{i j}$

$$
E=\left(\frac{N_{A}}{N_{A}+N_{B}}\right)^{2} \varepsilon_{A A}+\left(\frac{N_{B}}{N_{A}+N_{B}}\right)^{2} \varepsilon_{B B}+\frac{N_{A} N_{B}}{\left(N_{A}+N_{B}\right)^{2}} \varepsilon_{A B}
$$

The model fitting parameters $\varepsilon_{A A}, \varepsilon_{B B}, \varepsilon_{A B}$ and the volume per lattice site can be found by representing physical properties, such as heat capacity and density of the pure components and of the mixture.

Luettmer-Strathmann has extended this approach and applied it to mixtures in the non equilibrium state [90]. Our simulation box consists in this case of two equal chambers with slightly different temperatures (c.f. Fig. 1.3). The molecules of each type can be found then either in chamber I $\left(N_{A}^{I}, N_{B}^{I}\right)$ or in chamber $2\left(N_{A}^{I I}, N_{B}^{I I}\right)$. The partition function of the whole system is the product of the partition functions of the chambers $Z_{I} Z_{I I}$, summing over all possible configurations

$$
Q=\sum Z^{I}\left(\frac{N_{\text {total sites }}}{2}, T, N_{A}^{I}, N_{B}^{I}\right) Z^{I I}\left(\frac{N_{\text {total sites }}}{2}, T, N_{A}^{I I}, N_{B}^{I I}\right) .
$$


The average concentration $x_{A}^{I, I I}$ in the first (or second) chamber is given by

$$
x_{A}^{I, I I}=\frac{1}{Q} \sum \frac{N_{A}^{I, I I}}{N_{A}^{I, I I}+N_{B}^{I, I I}} Z^{I}\left(\frac{N_{\text {total sites }}}{2}, T, N_{A}^{I}, N_{B}^{I}\right) Z^{I I}\left(\frac{N_{\text {total sites }}}{2}, T, N_{A}^{I I}, N_{B}^{I I}\right) .
$$

Finally, the Soret coefficient of component A is calculated

$$
S_{\mathrm{T}}=-\frac{1}{x_{A}\left(1-x_{A}\right)} \frac{x_{A}^{I}-x_{A}^{I I}}{T^{I}-T^{I I}}
$$

where $x_{A}=N_{A}\left(N_{A}+N_{B}\right)$ is related to the whole system.

This model has been successfully applied to determine the Soret coefficient of ethanol in water [91] and PEO in water/ethanol mixtures [74].

\subsubsection{Molecular dynamics simulations}

Equilibrium molecular dynamic In the previous section we have described the two-chamber lattice model, which is based on general thermodynamical rules. The molecular dynamic method describes the behavior of a system on the molecular level. In this method Newton's equations of motion are solved. First we prepare a sample: we select a model system consisting of $N$ particles. We should assign initial positions and velocities to all particles in the system. Then we can calculate the force acting on a given particle

$$
\vec{f}=-\nabla U
$$

The potential functions representing the non-bonded energy are formulated as a sum over interactions between the particles of the system. The simplest choice, employed in many popular force fields, is the "pair potential", in which the total potential energy can be calculated from the sum of energy contributions between pairs of atoms. An example of such a pair potential is the non-bonded Lennard-Jones potential and Coulomb's law [54]

$$
U=4 \varepsilon_{i j}\left[\left(\frac{\sigma_{i j}}{r_{i j}}\right)^{12}+\left(\frac{\sigma_{i j}}{r_{i j}}\right)^{6}\right]+\frac{q_{i} q_{j}}{4 \pi r_{i j} \varepsilon \varepsilon_{0}},
$$

where $r_{i j}$ is the distance between two particles, $q_{i}$ and $\sigma_{i}$ - their charges and diameters, $\varepsilon_{0}$ and $\varepsilon$ are the vacuum permittivity and the effective dielectric constant. Lennard-Jones parameters for mixed interactions can be obtained from the Lorentz-Berthelot mixing rules [54]

$$
\varepsilon_{i j}=\sqrt{\varepsilon_{i i} \varepsilon_{j j}} \quad \sigma_{i j}=\frac{\sigma_{j j}+\sigma_{i i}}{2} .
$$


For describing the motion of the atoms belonged to the same molecule, one needs to consider additional contributions to the interaction potential.

$$
\begin{aligned}
& U_{\text {bond }}=\sum_{\text {bonds }} \frac{k_{r}}{2}\left(r-r_{0}\right)^{2}, \\
& U_{\text {angles }}=\sum_{\text {angles }} \frac{k_{\varphi}}{2}\left(\varphi-\varphi_{0}\right)^{2}, \\
& U_{\text {harmonic dihedral }}=\sum_{\text {harmonic dihedral }} \frac{k_{\delta}}{2}\left(\delta-\delta_{0}\right)^{2}, \\
& U_{\text {torsion }}=\sum_{\text {torsions }} \frac{k_{\tau}}{2}\left[1-\cos p\left(\tau-\tau_{0}\right)\right],
\end{aligned}
$$

where $r$ is the distance between two atoms , $\varphi$ is a bond angle, $\delta$ is a harmonic dihedral angle, $\tau$ is a torsional angle ( $p$ is the periodicity of the potential) and $k$ is the force constant. The subscript "0" identifies the equilibrium value. The position and velocity of each atom in a timestep $\triangle t$ can then be calculated using Newton's equations of motion

$$
v_{n+1 / 2}=v_{n-1 / 2}+\frac{\triangle t}{m} f_{n} \quad x_{n+1 / 2}=x_{n}+\triangle t v_{n+1 / 2}
$$

Equilibrium simulations we performed at constant pressure and temperature using the Berendsen's thermostat [16]

$$
\frac{d T}{d t}=\frac{1}{\tau_{T}}\left(T_{b a t h}-T\right) \quad \frac{d P}{d t}=\frac{1}{\tau_{P}}\left(P_{b a t h}-P\right),
$$

where $P$ and $T$ are the actual temperature and pressure of the system, $P_{\text {bath }}$ and $T_{\text {bath }}$ are the target values, $\tau_{\mathrm{P}}$ and $\tau_{\mathrm{T}}$ are characteristic times which determine how quickly the system reacts to a deviation from the target values. A constant temperature is regulated by a uniform scaling of the atom velocities and a constant pressure by a uniform scaling of the atom positions and the box lengths.

Reverse non equilibrium molecular dynamics (RNEMD) In order to calculate the Soret coefficient we need to impose the temperature gradient in our simulation box. This can be done using the so-called heat exchange algorithm (HEX) [65]. For all simulations 


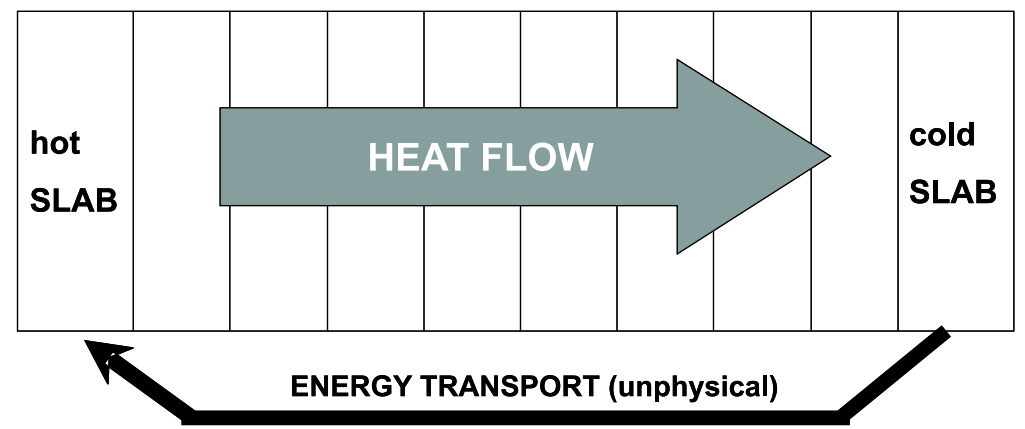

Figure 1.4: Illustration of the heat exchange algorithm for determination of the Soret coefficient by non equilibrium simulation.

we have used the YASP package, developed by Müller-Plathe [126]. The simulation box with periodic boundary conditions is divided in $n$ slabs. Fig. 1.4 shows the left half of the simulation box. The left slab designated as the hot slab and the right one - the cold slab. In the beginning our system is in equilibrium state and the temperatures of all slabs are equal. The temperature difference between the left and the right slabs is created by exchanging the Cartesian velocity vectors of the hottest particle of the cold slab and the coldest particle of the hot slab each $N_{\text {exch }}$ timestep of our RNEMD simulations. Due to the conservation of energy it leads to a heat flow $j_{z}$ through our simulation box. In the steady state, the magnitude of $j_{z}$ is equal to the imposed unphysical energy flow.

$$
j_{z}=\frac{1}{2 t A} \sum_{\text {transfer }} \frac{m}{2}\left(v_{\text {hot }}^{2}-v_{\text {cold }}^{2}\right),
$$

where $A$ is the cross sectional area of the simulation box perpendicular, $t$ is the length of the simulation, $v_{h o t}$ and $v_{\text {cold }}$ are the velocities of the hot and the cold particle of the same mass $m$, whose velocities are exchanged. For mixtures of molecules the Cartesian centre-of-mass velocity vectors of the two selected molecules need to be exchanged in order to keep their conformations. In this way the relative velocities of all atoms in the given molecule remain unchanged. The Cartesian centre-of-mass velocity vector is defined as

$$
\vec{v}_{c m}=\sum_{\substack{\text { all atoms in } \\ \text { molecule }}} m_{i} \vec{v}_{i}\left(\sum_{\substack{\text { all atoms in } \\ \text { molecule }}} m_{i}\right)^{-1},
$$


where $m_{i}$ and $v_{i}$ are masses and velocities of atoms in the given molecule. The temperature in a molecular dynamics simulation with constraints is given by the equipartition theorem

$$
\left(\frac{3 N-C}{2}\right) k_{B} T=\frac{1}{2}\left\langle\sum_{i} m_{i} v_{i}^{2}\right\rangle
$$

where $C$ is the number of constraints in the given slab. The Soret coefficient of a binary mixture can be calculated using Eq. 1.19 with the concentration gradient and the temperature gradients, obtained from simulations.

RNEMD simulations were performed for many kinds of simple mixtures. The equimolar mixtures of Lennard-Jones particles with different sizes, masses and the deeps of interaction potential were studied by Reith et al [126]. Later, a more realistic mixture of methane in "super" methane was studied by Galliero et al [56]. The properties such as mass, size and strength of interactions of the "super" methane were systematically varied being first similar to methane and then becoming more different. Artola et al. [9] investigated the concentration dependence of the Soret coefficient on the molecular interaction parameters for LJ mixture. Galliero et al. [55] used the LJ approximation to investigate the thermal diffusion behavior in pentane/decane mixture. Thermal diffusion behavior of methane/n-decane and pentane/ $n$-decane mixtures was investigated by Simon et al. [156] and Perronace at. al [113] using a united atom description of the alkanes. The Soret coefficient calculated for benzene/cyclohexane mixtures [189] and the mixtures of methanol, ethanol, acetone and dimethylsulfoxide (DMSO) in water [104] with a full atomistic description of the molecules are in satisfactory agreement with the experiment.

\subsection{Experimental methods}

In this section we present an overview of the different experimental methods for measuring the Soret coefficient. One needs highly sensitive experimental methods in order to work with extremely small temperature and concentration gradients. In order to determine the Soret coefficient, the steady state needs to be reached, which can take a long time if the cell dimensions are large and the mutual diffusion is slow. Those long measurement times in the order of days require an excellent stability of the experimental set-up. Often the thermal diffusion process is disturbed by convection effects. If in the experimental arrangement the convection can not be suppressed it needs to be accounted for. 


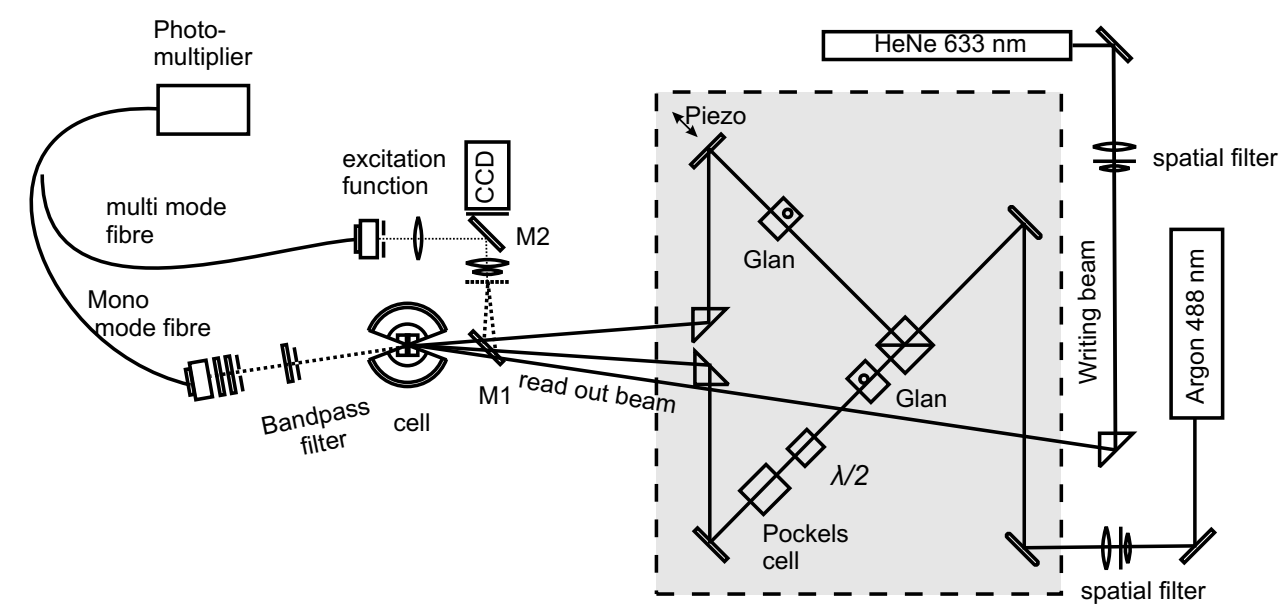

Figure 1.5: Schematic drawing of a thermal diffusion forced Rayleigh scattering (TDFRS) setup

\subsubsection{Thermal Diffusion Forced Rayleigh Scattering}

In 1978, Thyagarajan for the first time observed a fast heat and a slow concentration mode in a forced Rayleigh scattering experiments on a mixture of CS2 and ethanol [163]. During the last years, the optical holographic grating technique has been improved [76, 182, 129] and applied to study thermal diffusion behavior in simple and complex fluids. The experimental setup (c.f. Fig. 1.5) is mounted on an optical table with tuned damping. An argon-ion laser $(488 \mathrm{~nm})$ is used as writing beam. The beam is spatially filtered and expanded to a diameter of 5-10 mm. The polarization is perpendicular to the optical table. The beam is splitted into two beams of equal intensity with a beam splitter. Glan prisms are used to refine the polarization for better contrast. A mirror is mounted on piezo ceramics, which is used for phase stabilization and phase modulation of the grating. The Pockels cell and the half wave plate are used to shift the grating by $180^{\circ}$. The writing beams are reflected by two prisms towards the sample cell. The positions of the two prism and the distance to the intersection point of the two beams determine the grating vector $q$. The angle $\theta$ between the two writing beams is typically between $2-4^{\circ}$. Such a small angle is measured by imaging the interference grating directly on a CCD camera using the flip mirror M1. Analyzing the distance between 
the fringes allows to determinate the grating vector $q=4 \pi \sin (\theta / 2) / \lambda$ with an accuracy of $0.5 \%$. The mirror M2 in front of the CCD camera reflects the grating directly on the photomultiplier. By selecting one of the interference stripes the excitation function can be measured directly. Generally, the excitation is not ideal and takes $\approx 10 \mu$ s to rise up to $90 \%$ of the plateau value and the final plateau is reached after $160 \mathrm{~ms}$ due to the finite switching time of the Pockels cell. The measured excitation function is used for correction of the measured TDFRS signal [109].

The sample cell is mounted inside a brass holder and can be adjusted in directions orthogonal to the optical axis. A quartz cell (Hellma) with a layer thickness of $0.2 \mathrm{~mm}$, is used for TDFRS measurements. The temperature of the brass holder is controlled by a circulating water bath from a thermostat (Lauda) with an uncertainty of $0.02{ }^{\circ} \mathrm{C}$. By using an external temperature sensor, the thermostat controls the temperature in the cell. The diffraction efficiency of the refractive index grating in the sample cell is read by a He-Ne laser with a wavelength $\lambda=632.8 \mathrm{~nm}$ at the Bragg angle. A pinhole and bandpass filter in front of the detector separate the diffracted beam from stray light and the light of the writing beams. A single mode fiber is directly connected to the photomultiplier tube operating in photon counting mode (c.f. Fig.1.5).

The measured intensity $I$ in the TDFRS experiment contains contributions from the electric field amplitude of the diffracted beam $E_{\mathrm{S}}$, the coherent electric field amplitude $E_{\mathrm{c}}$ and the incoherent electric field amplitude $E_{\text {inc }}$

$$
I=\left|E_{\mathrm{c}}+E_{\mathrm{s}} e^{i \phi}\right|^{2}+E_{\mathrm{inc}}^{2}=E_{s}^{2}+2 E_{\mathrm{s}} E_{\mathrm{c}} \cos \phi+E_{c}^{2}+E_{\mathrm{inc}}^{2}
$$

where $\phi$ is the phase shift between the signal and the coherent background. The background from incoherent scattering can be completely suppressed by heterodyne $\left(S_{\text {het }}\right)$ signal detection

$$
S_{\text {het }}=\frac{1}{2}\left(I_{\phi}-I_{\phi+\pi}\right)=2 E_{\mathrm{c}} E_{\mathrm{s}} \cos \phi .
$$

Due to this reason in actual TDFRS experiments, the heterodyne detection is always superior to the homodyne $\left(S_{\text {hom }}\right)$ [129]

$$
S_{\text {hom }}=\frac{1}{2}\left(I_{\phi}+I_{\phi+\pi}\right)=E_{\mathrm{s}}^{2}+E_{\mathrm{c}}^{2}+E_{\mathrm{inc}}^{2} .
$$

Working equations An optical grating is created by the interference of two writing beams operating at the wavelength $\lambda=488 \mathrm{~nm}$. The dye with a strong adsorption band at this wave- 
length converts the optical grating into a temperature grating. The evolution of the temperature grating can be described as

$$
\frac{\partial T(x, t)}{\partial t}=D_{\mathrm{th}} \frac{\partial^{2}}{\partial x^{2}} T(x, t)+S(x, t)
$$

where $D_{\text {th }}$ is thermal diffusivity and the source term $S(x, t)$ is given by

$$
S(x, t)=\frac{\alpha}{\rho c_{\mathrm{p}}} I(x, t)=S_{0}+S_{q}(t) e^{i q x}
$$

where $\alpha$ is an optical absorption coefficient, $c_{\mathrm{p}}$ the specific heat at constant pressure, $\rho$ is the density and $I(x, t)$ is the intensity of the writing beam. Eq. (1.57) is solved by

$$
T(x, t)=T_{0}+T_{m}(t)+T_{q}(t) e^{i q x}
$$

where $T_{0}$ is the initial sample temperature and $T_{m}(t)=\alpha I_{0} t / \rho c_{\mathrm{p}}$ is the mean sample temperature. The amplitude $T_{q}(t)$ of the temperature grating is expressed as a linear response for arbitrary excitations $S_{q}(t)=\alpha\left(\rho c_{\mathrm{p}}\right)^{-1} I_{q}(t)$ :

$$
T_{q}(t)=\int_{-\infty}^{t} d t^{\prime} S_{q}\left(t^{\prime}\right) e^{-\left(t-t^{\prime}\right) / \tau_{\mathrm{th}}}
$$

where $\tau_{\mathrm{th}}=\left(D_{\mathrm{th}} q^{2}\right)^{-1}$ is the decay time for the heat diffusion, after which a stable temperature grating is reached.

The build-up of the concentration grating due to the Ludwig-Soret effect in a fluid mixture can be evaluated from the one-dimensional diffusion equation

$$
\frac{\partial c(x, t)}{\partial t}=D \frac{\partial^{2}}{\partial x^{2}} c(x, t)+D_{\mathrm{T}} c_{0}\left(1-c_{0}\right) \frac{\partial^{2}}{\partial x^{2}} T(t, x)
$$

with the solution

$$
c(x, t)=c_{0}+c_{q}(t) e^{i q x}
$$

where

$$
c_{q}(t)=-q^{2} D_{\mathrm{T}} c_{0}\left(1-c_{0}\right) \int_{-\infty}^{t} d t^{\prime} T_{q}\left(t^{\prime}\right) e^{-\left(t-t^{\prime}\right) / \tau} .
$$

with $\tau$ the decay time associated with the collective diffusion. The resulting refractive index grating can be expressed as

$$
n(x, t)-n_{0}=n_{q}(t) e^{i q x}=\left[\left(\frac{\partial n}{\partial T}\right)_{c, p} T_{q}(t)+\left(\frac{\partial n}{\partial c}\right)_{T, p} c_{q}(t)\right] e^{i q x}
$$


where $n_{0}$ is the refractive index at the readout wavelength $(633 \mathrm{~nm})$. The heterodyne diffraction signal $\zeta_{\text {het }}(t)$ is proportional to the refractive index modulation depth:

$$
\zeta_{\text {het }} \propto E_{\mathrm{c}} E_{\mathrm{S}} \cos \phi \propto n_{q}(t)
$$

Combining Eqs.1.59, 1.60, 1.63, 1.64, 1.65, the heterodyne signal can be evaluated as

$$
\zeta_{\text {het }}=1-e^{-t / \tau_{\mathrm{th}}}-A\left(\tau-\tau_{\mathrm{th}}\right)^{-1}\left[\tau\left(1-e^{-t / \tau}\right)-\tau_{\mathrm{th}}\left(1-e^{-t / \tau_{\mathrm{th}}}\right)\right]
$$

where $A$ is the ratio of the steady-state amplitudes of the concentration grating $\zeta_{\mathrm{c}}(t \rightarrow \infty)$ to the thermal contribution $\zeta_{\text {th }}(t \rightarrow \infty)$ :

$$
A=\frac{\zeta_{c}(t \rightarrow \infty)}{\zeta_{\mathrm{th}}(t \rightarrow \infty)}=\left(\frac{\partial n}{\partial c}\right)_{p, T}\left(\frac{\partial n}{\partial T}\right)_{p, c}^{-1} S_{\mathrm{T}} c_{0}\left(1-c_{0}\right)
$$

Using the fact that the build-up of the temperature grating is much faster than the build-up of the concentration grating, Eq.1.66 can be simplified, by using $\tau_{\text {th }} \ll \tau$, to

$$
\zeta_{\text {het }}=1-e^{-t / \tau_{\text {th }}}-\left(\frac{\partial n}{\partial c}\right)_{p, T}\left(\frac{\partial n}{\partial T}\right)_{p, c}^{-1} S_{\mathrm{T}} c_{0}\left(1-c_{0}\right)\left(1-e^{-t / \tau}\right) .
$$

Eq.1.68 is fitted the experimental heterodyne diffraction signal and determined the transport coefficients $D_{\mathrm{th}}, D, D_{\mathrm{T}}$ and Soret coefficient $S_{\mathrm{T}}$. The two contrast factors $(\partial n / \partial T)_{c, p}$ and $(\partial n / \partial c)_{T, p}$ have to be obtained separately.

Contrast factors The contrast factors $(\partial n / \partial c)_{T, p}$ is measured by an Abbe refractometer at $589 \mathrm{~nm}$ with further correction for the wavelength of the readout laser $(633 \mathrm{~nm})$. The contrast factor $(\partial n / \partial T)_{c, p}$ was measured with a Michelson interferometer at $633 \mathrm{~nm}$. Figure 1.6 shows a sketch of the $(\partial n / \partial T)$-setup. Two foil polarizers are used to adjust the intensity. The laser beam is splitted into two beams. One beam goes through the beam splitter to the measurement cell and is reflected at the windows of the measurement cell. The reflected beam at the front window (a, b) and at the back window $(\mathbf{c}, \mathbf{d})$ are superposed at the photodiode. The main contribution of the reflections stem from $\mathbf{a}$ and $\mathbf{d}$ due to the larger refractive index differences $(\sim 0.5)$ to air compared to the smaller refractive index differences at $\mathbf{b}$ and $\mathbf{c}$ $(\sim 0.01)$ at the inner window, which is in contact with the liquid. The optical path difference depends on the change of the refractive index $n$ and $n_{\mathrm{w}}$ and the length $l$ and $l_{\mathrm{w}}$ of the sample and the window, respectively

$$
d s=d(n l)+d\left(2 n_{w} l_{w}\right) .
$$




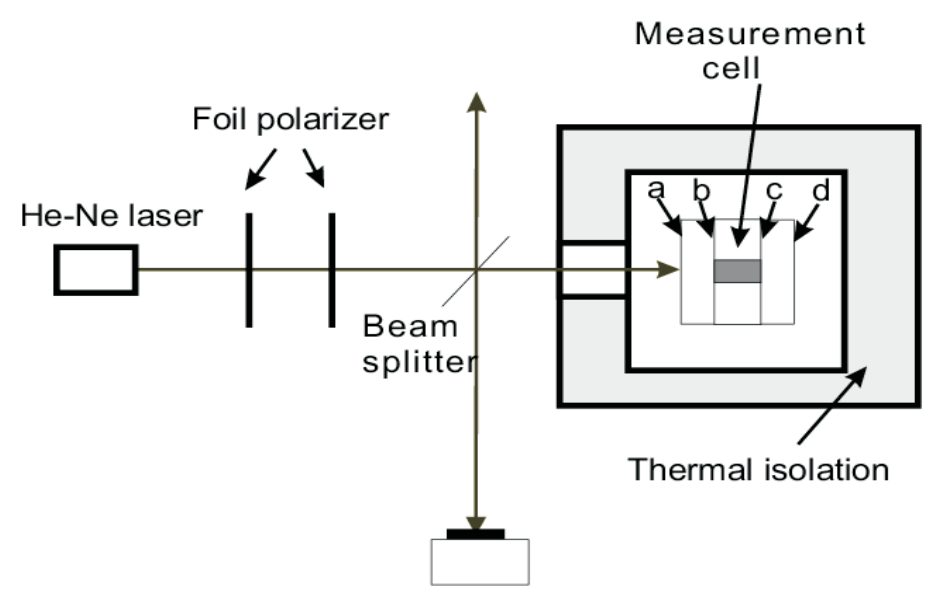

Photo diode

Figure 1.6: Sketch of the $\partial \mathrm{n} / \partial \mathrm{T}$ interferometer

The temperature derivative of refractive index is obtained by,

$$
\frac{\partial n}{\partial T}=\frac{1}{2 k l} \cdot \frac{\partial \phi}{\partial T}-2 \cdot \frac{n_{w}}{l} \cdot \frac{\partial l_{w}}{\partial T}-2 \cdot \frac{l_{w}}{l} \cdot \frac{\partial n_{w}}{\partial T}-\frac{n}{l} \cdot \frac{\partial l}{\partial T} .
$$

For this setup, $n_{w}=1.457$. The thermal expansion coefficients $\left(1 / l_{w}\right) \cdot\left(\partial l_{w} / \partial T\right)$ and $(1 / l)$. $(\partial l / \partial T)$ are $5.1 \mathrm{E}-7 \mathrm{~K}^{-1}$ and $7.5 \mathrm{E}-7 \mathrm{~K}^{-1}$, respectively, and $2 \cdot\left(l_{w} / l\right) \cdot\left(\partial n_{w} / \partial T\right)$ is $2.45 \mathrm{E}-$ $6 \mathrm{~K}^{-1}$ [Ref. 182].

Validation of TDFRS method TDFRS method was validated for three binary mixtures of simple molecules in a benchmark test [116]. Generally, it was applied to different kind of mixtures: simple fluid mixtures [36, 113, 75, 111, 183, 185] polymer solutions [34, 72, 123], micellar solutions $[109,110]$ and colloidal dispersions $[33,106]$. The advantages of the method are a small temperature difference $(\sim 20 \mu \mathrm{K})$ and a small fringe spacing $(\sim 20 \mu \mathrm{m})$ which keeps the system close to the thermal equilibrium and allows also the investigation of slow diffusing systems such as polymers and colloids. On the other hand it works also for low molecular weight mixtures. This technique can also be applied to ternary mixtures, when one of the components diffuses much slower than the others [74]. The main disadvantage is that for some associated mixtures the addition of a small amount of inert dye, which converts the optical grating in a temperature grating, can sometimes influence the observed thermal 
diffusion behavior [33, 109].

\subsubsection{Thermal lens method}

The thermal lens (TL) effect is nowadays widely used in microscopy [99], absorption spectrometry [22], in analysis of trace components in gas - and liquid - phase samples [88], for investigation of the population redistribution between excited and ground levels in ion-doped materials [120], etc. This effect was first observed by Gordon et al. [62] in a liquid placed within the resonator of a helium-neon laser. Later, Giglio and Verdramini [59] noticed that the thermal lens in a binary mixture was noticeably larger than in pure components. The first full theoretical description of the thermal lens effect was done by Sheldon et al. [152]. Different types of TL techniques (one or two beam configuration and open or closed aperture) have been developed [83]. In this work we consider a classical one beam thermal lens setup with closed aperture of the detector for investigation of the thermal diffusion in liquids.

In a thermal lens experiment the Gaussian laser beam is used for both heating and detection simultaneously. The uniform local heating of the partially absorbing medium by the laser beam creates a lens due to the dependence of the refractive index on temperature. Typically, this lens is concave because $\partial n / \partial T$ is negative for liquids (c.f. Fig. 1.9). In a binary mixture additionally a Soret lens is formed due to the dependence of the refractive index on concentration. The physical properties of the formed lenses can be probed by measuring the intensity of the central beam with time or by measuring the intensity as function of the distance $z$ between the sample and the beam waist. Analyzing the data the Soret, thermal diffusion and mutual diffusion coefficients can be calculated.

The basic equations The temperature change in the sample as a function of radius and time $\triangle T(r, t)$ can be obtained from the heat equation

$$
c \rho \frac{\partial}{\partial t} \triangle T(r, t)=\dot{q}(r)+\lambda \nabla^{2} \triangle T(r, t),
$$

where $\lambda$ is the thermal conductivity, $c$ is the heat capacity, $\rho$ is the density and $\dot{q}(r)$ is the heat source term. The source term can be calculated from the changing of the intensity of the Gaussian beam passed through the sample with a thickness $l$ and absorbtion $b$

$$
\dot{q}(r)=\frac{\triangle I}{l} \approx I_{0} b=\frac{0.48 P b}{\pi \omega^{2}} \exp \left(\frac{-2 r^{2}}{\omega^{2}}\right),
$$



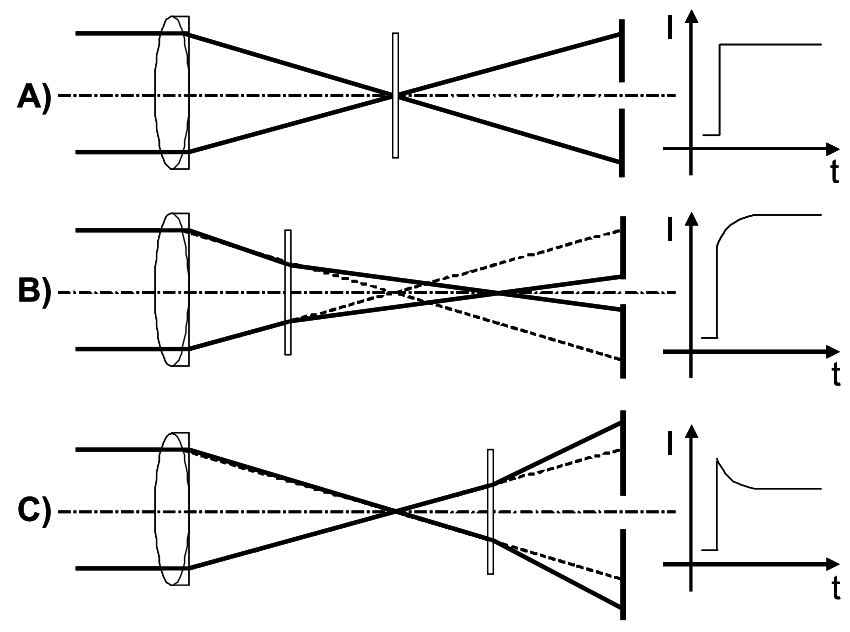

Figure 1.7: Change in beam divergence of a sample with negative $\partial n / \partial T$, which moves through the beam waist. The dashed lines show the divergence of the beam without the presence of a nonlinear sample.

where $P$ is the power of the laser and $\omega$ is the beam diameter. The solution of Eq. 1.71 is

$$
\triangle T(r, t)=\frac{0.48 P}{\pi c \rho \omega^{2}} \int_{0}^{t}\left(\frac{1}{1+2 t / \tau_{t h}}\right) \exp \left(\frac{-2 r^{2} / \omega^{2}}{1+2 t / \tau_{t h}}\right) d t .
$$

The temperature gradient forms during a characteristic time $\tau_{t h}$

$$
\tau_{t h}=\frac{\omega^{2}}{4 D_{t h}}
$$

which is determined by the size of the beam and the diffusivity $D_{t h}$ of the sample.

The central beam intensity can be calculated using diffraction integral theory. The complex phase amplitude of the wave after the pinhole $U_{P}(r, t)$ (c.f. Fig. 1.8) is a result of the superpositions of Huygens spherical waves $U_{\text {spherical }}(r, t)$

$$
U_{p}(t)=\frac{i}{\lambda} \int_{0}^{\infty} \int_{0}^{2 \pi} U_{\text {spher }}(r, t)\left(\frac{1+\cos \alpha}{2}\right) \frac{\exp [-i(2 \pi / \lambda)|\vec{R}-\vec{r}|]}{|\vec{R}-\vec{r}|} r d r d \theta .
$$

The phase of the spherical wave is determined by the distance between the source of the spherical wave and the detector, which can be approximated

$$
\frac{2 \pi}{\lambda} \sqrt{r^{2}+R^{2}} \approx \frac{2 \pi}{\lambda}\left(R+\frac{r^{2}}{2 R}\right) \text {. }
$$




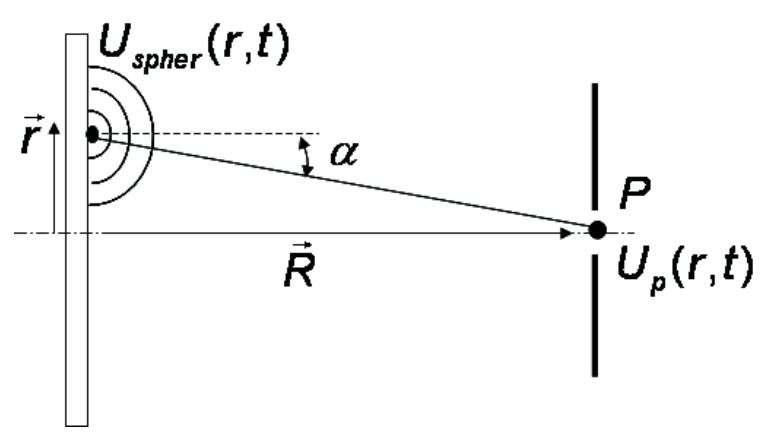

Figure 1.8: To the calculation of the intensity after pinhole. $\mathrm{P}$ is the position of detector.

and by the optical path variation along $\vec{r}$

$$
\frac{2 \pi}{\lambda} l[n(r, T)-n(0, T)] \approx \frac{2 \pi}{\lambda} n_{0} l+\frac{2 \pi}{\lambda} \frac{d n}{d T} l[\triangle T(0, t)-\triangle T(r, t)] .
$$

The spherical wave can be written as

$$
U_{\text {spher }}(r, t)=A \exp \left(-\frac{r^{2}}{\omega^{2}}\right) \exp \left(-\frac{i \pi}{\lambda}\left(\frac{r^{2}}{R}+2 l \frac{d n}{d T}[\triangle T(0, t)-\triangle T(r, t)]\right)\right) .
$$

Combining Eq. $1.73,1.75,1.78$ and considering $|\vec{R}-\vec{r}| \approx R$ the intensity at the detector position after pinhole $I=\left|U_{p}(t)\right|^{2}$ can be calculated

$$
I(t)=I(0)\left(1+f\left(\theta_{t h}, \gamma, \tau_{t h}, t\right)\right) ; \quad f=A \theta_{t h}+B \theta_{t h}^{2} .
$$

The dimensionless parameter $\gamma$ characterizes the distance from the cell to the beam waist. The parameter $\theta_{t h}$ characterizes the strength of the thermal lens and is given by

$$
\theta_{t h}=-\frac{0.52 P b l}{\kappa \lambda} \frac{\partial n}{\partial T}
$$

The coefficients A and B are given by Eq. 1.81 and 1.82, respectively.

$$
\begin{aligned}
& A=-\operatorname{atan}\left[\frac{2 \gamma}{3+\gamma^{2}+\left(9+\gamma^{2}\right) \tau_{t h} / 2 t}\right] \\
& B=\frac{A^{2}}{4}+\left(\frac{1}{4} \ln \left[\frac{\left[\left(2+\tau_{t h} / t\right)\left(3+\gamma^{2}\right)+6 \tau_{t h} / t\right]^{2}+16 \gamma^{2}}{\left(9+\gamma^{2}\right)\left(2+\tau_{t h} / t\right)^{2}}\right]\right)^{2} .
\end{aligned}
$$


The Soret lens is described by the same equations. In order to take into account the Soret effect an additional term $\left.f\left(\theta_{\text {Soret }}, \gamma, \tau_{\text {Soret }}, \mathrm{t}\right)\right)$ needs to be added to Eq. 1.79 . Then the Soret coefficient $S_{\mathrm{T}}$ for a binary mixture with concentration c is given by

$$
S_{\mathrm{T}}=-\frac{\theta_{\text {Soret }}}{\theta_{t h}} \frac{\partial n / \partial T}{\partial n / \partial c} c^{-1}(1-c)^{-1}
$$

The experimental measured signal $I(\gamma, t)$ (time or coordinate dependence with respect to the beam waist) is fitted using Eq. 1.79. Then the thermal conductivity $(\kappa)$ or the Soret coefficient $\left(S_{\mathrm{T}}\right)$ can be calculated (c.f. Eq. 1.80 or 1.83 , respectively).

Validation of thermal lens method The equilibration time in the thermal lens method is relative short compared to the diffusion cell due to small distances in the order of the focal beam width, which makes it possible to investigate slow diffusing systems (polymer solutions or colloidal dispersions). So far the thermal lens method has not been validated in a benchmark. However, it has been used to study the thermal diffusion behavior of ferrofluids [7] and ionic surfactant systems [137]. These studies showed agreement with forced Rayleigh scattering and beam deflection measurements, respectively. In contrast, Voit [172] did not find agreement with other methods. For one of the benchmark mixtures $n$-dodecane/1,2,3,4 tetrahydronaphthalene, Voit found a $40 \%$ too small value, which was probably due to convection.

The main advantage of the method is that we can avoid the addition of a dye for aqueous systems by using an infrared laser, as far as water shows a weak absorption band at a wavelength $\lambda=980 \mathrm{~nm}$. The main disadvantage is the sensitivity to convection, astigmatism of the beam and the fact that no single scattering vector $q$ can be selected, as in the case of the grating experiments.

\subsubsection{Thermogravitational column}

Thermogravitational column is one of the oldest methods for the measurement of the thermal diffusion coefficient. Fig. 1.9 shows the sketch of the experimental setup. Thermogravitational column consists of two hollow tubes connected to each other on top and bottom. The gap between them is filled by the investigated mixture. Each tube is maintained at constant but different temperatures, establishing the temperature gradient in the investigated mixture. The denser component migrates to the cold wall due to the thermal diffusion effect and then to the 


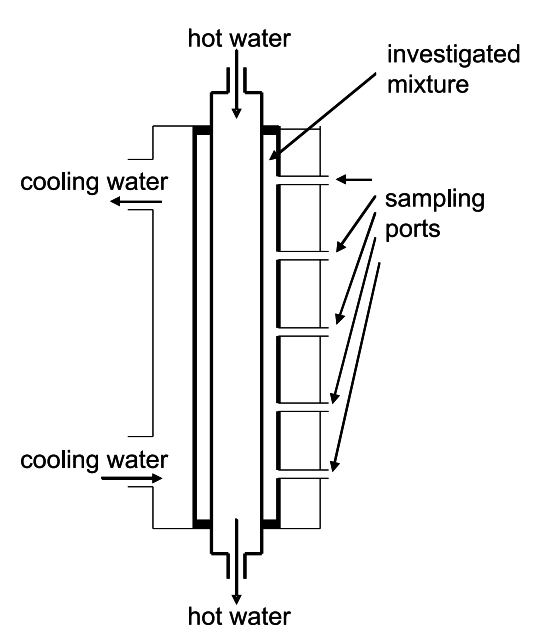

Figure 1.9: the sketch of the thermogravitational column.

bottom due to convection. After the stationary state is reached, samples are taken from five sampling ports and their concentration is determined by density measurements. The obtained difference in concentration $\triangle c$ between bottom and top is used for calculation of the thermal diffusion coefficient [45]

$$
D_{T}=\frac{\alpha g \triangle c}{504 v c(1-c)} \frac{L_{x}^{4}}{L_{z}},
$$

where $v$ is the kinematic viscosity, $\alpha$ is the thermal expansion coefficient, $g$ is the gravity acceleration, $L_{z}$ is the height of the column and $L_{x}$ is the gap between the two tubes. Due to the fact that $D_{\mathrm{T}}$ depends on $L_{x}$ to the power of four precise measurement of $L_{x}$ and its uniformity along the entire length of the column are required. Also the characteristic relaxation time $t_{r}$ depends strongly on $L_{x}[45]$

$$
t_{r}=\frac{9 ! L z^{2} v^{2} D}{\left(\pi \triangle T g \alpha L_{x}^{3}\right)^{2}}
$$

Typical experimental conditions are a temperature difference of $\triangle T=5^{\circ} \mathrm{C}$, a gap of $L_{x}=2$ mm. With a diffusion coefficient of $D=4 \times 10^{-6} \mathrm{~cm}^{2} s^{-1}$ this leads to a relaxation time of $t_{r} \approx 30 \mathrm{~min}$. For comparison in a TDFRS and TL setup the steady state is reached after 100 $\mathrm{ms}$ and $100 \mathrm{~s}$, respectively. 


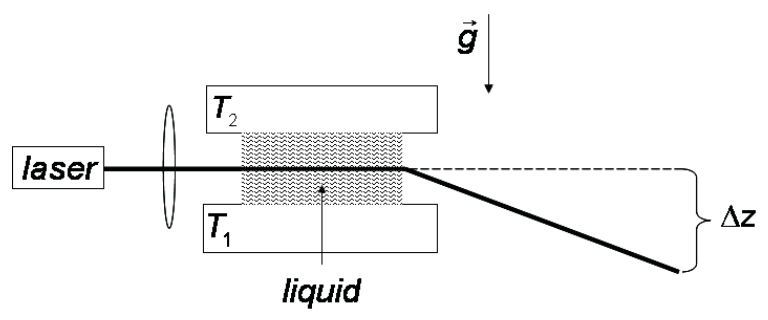

Figure 1.10: Schematic diagram of the thermal diffusion cell method.

Since 1949 thermogravitational columns have been employed to determine thermal diffusion coefficient for different mixtures [167, 159, 92, 165, 144]. However, in those older studies the results were often not consistent and often only quantitative information about the thermal diffusion factor could be obtained. The main reasons are a lack of a precise theory and convection problems. Another difficulty is due to the small values of $S_{\mathrm{T}}$ (in the order of $10^{-3} \mathrm{~K}^{-1}$ for low molecular weight mixtures), the resulting mass fraction gradient is usually very small (less than $1 \%$ mass fraction difference between two walls). In the last decade this technique was significantly improved and shows now satisfactory agreement with other experimental methods for the mixture toluene/ $n$-hexane [20] and the three binary mixtures of 1,2,3,4-tetrahydronaphtalene, isobutylbenzene and $n$-dodecane [116].

\subsubsection{Thermal diffusion cells}

A thermal diffusion cell is a traditional experimental method for measuring the Soret coefficient $[48,178,95,102,60]$. Fig. 1.10 shows a sketch of the experimental setup. The heating of the investigated liquid from above and cooling from bellow leads to a concentration gradient in the vertical direction due to thermal diffusion. The laser beam propagates horizontally through the liquid mixture. The Soret and the mutual diffusion coefficients are calculated from the time dependence of the laser beam deflection $\triangle Z$ [17]

$$
\triangle Z_{\text {staionary }}(t)=l_{1} L \frac{T_{2}-T_{1}}{h}\left[\frac{d n}{d T}-S_{\mathrm{T}} c(1-c) \frac{d n}{d c}\left(1-\frac{4}{\pi} \exp \left(-t / \tau_{D}\right)\right)\right]
$$

where $l_{1}$ is the liquid path length, $h$ is the height of the liquid, $L$ is the distance from the cell to the detector and $\tau_{D}=h^{2} / \pi^{2} D$ is the equilibration time. For low molecular weight mixtures 
and a typical height of $2 \mathrm{~mm}$, the equilibration time is $\tau_{D} \approx 10 \mathrm{~min}$. This technique has been used to investigate toluene/ $n$-hexane [188] and ethanol/water [78, 188] mixtures. The obtained results are in a good agreement with other techniques.

\subsection{Overview of binary simple liquid mixtures}

So far there is no microscopic understanding of the Soret effect. Apparently, the magnitude as well as the sign of $S_{\mathrm{T}}$ are very sensitive to the chosen mixture. Generally, there is no Soret effect in the mixture of absolutely equal components due to the principle of symmetry. The Soret effect is basically the response of the system to the difference between two mixing partners. This simple conception was investigated in detail by experiments and by simulations.

The Soret coefficient of equimolar mixtures Molecular dynamics simulations of equimolar mixtures of particles [126] and spherical molecules [56] show that the component with the larger mass, the smaller radius and the larger depth of the interaction potential moves to the cold side. However, the experimental investigation of fairly simple equimolar mixtures without specific interactions show clearly that this simple relation between the Soret coefficient and physical properties of the components is not general [179]. The depth of the interaction potential was associated with the difference in the solubility (Hildebrandt) parameter $\delta$ of the two mixing partners. The Hildebrandt parameter can be estimated according to $\delta=\sqrt{\left(\rho\left(H_{v}-R T\right)\right) /(M)}$ with the gas constant $R$ and vaporization enthalpy $H_{v}$. The Hildebrandt parameter concept works well for the binary mixtures of cis-declin, 2-methylbutane and cyclohexane. For these mixtures the component with larger mass and larger density also moves to the cold. On the other hand the solubility concept fails for the binary mixtures of 1,2,3,4-tetrahydronaphthalene, $n$-dodecane and isobutylbenzene. For those three binary mixtures the component with the larger density moves to the cold side, but not the component with the larger mass.

The mass effect was investigated in more detail for different binary isotopic mixtures of the same substance. The measured Soret coefficient for chlorbenzene/chlorbenzene [139], brombenzene/brombenzene [139] and benzene/benzene [140] mixtures with different degree of the isotopic substitution can be described by a simple phenomenological expression con- 
sisting of two additive terms. The first term is due to the difference in mass $\delta M$ and the second one is due to the difference in the moment of inertia $\delta I$ between two isotopically substituted molecules of the same type

$$
S_{\mathrm{T}}=a_{M} \delta M+b_{I} \delta I
$$

The same holds also for four isotopically substituted CO and two $N_{2}$ systems [19] but with slightly different values of $a_{M}$ and $b_{I}$.

\section{Concentration dependence of the Soret coefficient}

Debuschewitz et al. [36] investigated experimentally mixtures of benzene isotopes in isotopes of cyclohexane. They considered an additional third term in Eq. 1.87 the so called chemical contribution $S_{\mathrm{T}}^{0}$ in order to describe the experimental data. The chemical contribution is a function of concentration in contrast to the mass and the moment of inertia terms. Wittko et al. [183] measured also the isotope effect $\triangle S_{\mathrm{T}}$, which is the change of $S_{\mathrm{T}}$ after isotopic substitution of cyclohexane $\left(\mathrm{C}_{6} \mathrm{H}_{12}\right.$ to $\left.\mathrm{C}_{6} \mathrm{D}_{12}\right)$ in benzene, hexane, toluene, 1,2,3,4tetrahydronaphtalene, isobutylbenzene, 1,6-dibromohexane and acetone. The obtained value $\triangle S_{\mathrm{T}} \approx 0.9910^{-3} \mathrm{~K}^{-1}$ neither depends on concentration nor on the mixing partner. Only in case of the polar acetone $\triangle S_{\mathrm{T}}$ is approximately $30 \%$ larger but still concentration independent. The isotope effect is not only independent of composition but also of temperature [185].

Many mixtures of simple molecules show a weak concentration dependence of the Soret coefficient in comparison to the associated ones. The dependence of $S_{\mathrm{T}}$ on concentration for carbon tetrachloride in methanol and ethanol is non-monotonic and $S_{\mathrm{T}}$ is equal to zero in carbon tetrachloride rich region [20]. Other associated mixtures like benzene/methanol [69], methanol/water [164], ethanol/water [74], acetone/water [111], dimehtylsulfoxide water [111], benzene/methanol show a sign change of $S_{\mathrm{T}}$ with concentration. For aqueous solutions, it was noted $[74,111]$ that the sign change concentration correlates with concentration, where the hydrogen bond network breaks down by addition of the second non-aqueous component.

MD simulations for LJ mixture of equal mass and equal size [9] as well as lattice calculation for ethanol/water mixture $[90,91]$ show that the slope $S_{\mathrm{T}}(x)$ as well as the sign change concentration are guided by the ratios of the interaction parameters $\varepsilon_{11} / \varepsilon_{12}$ and $\varepsilon_{22} / \varepsilon_{12}$. MD simulations were also performed for the mixtures of non-spherical molecules with taking into account their architecture and internal motion. The obtained results for $n$-pentane/ $n$-decane 
[113], benzene/cyclohexane [189], and the associated fluid mixtures of methanol, ethanol, acetone and dimethylsulfoxide (DMSO) in water [104] are in satisfactory agreement with the experimental data.

\section{Temperature dependence of the Soret coefficient}

Conceptually, the binary organic liquid mixtures can be divided into three groups according to the dependence of $S_{\mathrm{T}}$ on temperature.

In the entire concentration range for the mixtures from the first group the Soret coefficient becomes weaker at higher temperatures. Such behavior was observed for benzene/methanol mixture [69]. This system shows a non-monotonic concentration dependence of $S_{\mathrm{T}}$, which is retained with increasing temperature. The benzene concentration at which $S_{\mathrm{T}}$ changes its sign increases with temperature. The dibromohexane/cyclohexane mixture [185] can also be attributed to this group, while $S_{\mathrm{T}}$ does not change its sign with concentration.

For the mixtures from the second group the magnitude (not the value) of the Soret coefficient becomes weaker at higher temperature in a whole concentration range. This effect was found by Kolodner et al. [78] for ethanol/water mixture. The water concentration at which $S_{\mathrm{T}}$ changes its sign is temperature invariant.

All systems from the third group show a temperature invariant point, which means that $S_{\mathrm{T}}$ is not sensitive to the temperature but the corresponding value of $S_{\mathrm{T}}$ is not equal to zero. Toluene/ $n$-hexane [188] and benzene/cyclohexane [185] mixtures are related to this group.

\subsection{Outline of the thesis}

The aim of this thesis is to gain a better understanding of the thermal diffusion behavior in liquid mixtures. First, we investigated liquid binary systems of spherical, chain-like and associated simple molecules by thermal diffusion forced Rayleigh scattering method (TDFRS). The influence of physical properties like mass, density, Hildebrandt parameter etc. on $S_{\mathrm{T}}$ is analyzed. For some systems the obtained data were compared with the results from other experimental methods. Particular attention has been given to the validation of thermal lens (TL) technique for complex mixtures. The weak sides of both TDFRS method (the presence of the dye) and TL technique (convection problems) are discussed. Secondly, the Soret coefficient for some mixtures of spherical and chain-like molecules was calculated using the RNEMD 
method. Obtained data for chain-like mixtures were also compared with recent simulation results from Luettmer-Strathman. The thesis is organized as follows.

In Chapter 2, we report TDFRS measurements on three binary mixtures of spherical molecules (carbon tetrabromide, tetraethylsilane and di-tert-butylsilane in carbon tetrachloride). The results are discussed in the framework of thermodynamic theories and the Hildebrand parameter concept. Additionally, we determined the Soret coefficients for both silane/ carbon tetrachloride systems by reverse non-equilibrium molecular-dynamics calculations. The agreement between experiment and simulations is discussed.

In Chapter 3, we study the thermal diffusion behavior of $n$-decane in various alkanes by TDFRS method. The obtained results are compared with the measurements from thermogravitational column techniques. Typically the agreement between the two methods is in the order of $5 \%$. We analyze and discuss the possible reasons for the discrepancies.

In Chapter 4, the thermal diffusion behavior of binary mixtures of linear and branched alkanes in benzene is investigated by TDFRS for a range of concentrations and temperatures. The magnitude of the Soret coefficient decreases with increasing chain length, degree of branching and alkane content. The model, developed by Luettmer-Strathmann, quantitatively describes the behavior of the linear alkanes but not of the branched ones. The dependence of $S_{\mathrm{T}}$ on branching is not expected from the thermodynamic properties of the pure alkane fluids, which are the key parameters of the model.

In Chapter 5, the RNEMD method is applied for the investigation of the thermal diffusion of heptane and its isomers in benzene. We investigated the concentration dependence of the Soret coefficient for heptane in benzene and the effect of the degree of branching for equimolar heptane/benzene solutions. Compared to the experimental data, the simulation results show the same trend versus the molar fraction and the degree of branching. The influence the degree of branching on $S_{\mathrm{T}}$ was attributed to the packing effect and kinetic properties of the branched heptane isomers.

In Chapter 6, we performed systematic temperature and concentration dependent measurements of the Soret coefficient in different aqueous and non-aqueous associated binary mixtures using TDFRS. The influence of hydrophilic and hydrophobic interactions as well as the solubility on the sign change concentration of $S_{\mathrm{T}}$ is analyzed.

In Chapter 7, we present a thermal lens (TL) setup. Considering, that TL was not validated 
for a benchmark test we investigated different kinds of simple and complex mixtures with different orders of magnitude of the Soret coefficient $\left(10^{-3} \mathrm{~K}^{-1} 10^{-2} \mathrm{~K}^{-1}\right.$ and $\left.10^{-1} \mathrm{~K}^{-1}\right)$. The measured $S_{\mathrm{T}}$ values were compared with the results from TDFRS method. Additionally, the influence of dye (basantol yellow) on the Soret coefficient for $\mathrm{C}_{12} \mathrm{E}_{6}$ /water mixture was investigated with the TL setup. We did not observed the second slow mode found previously in the concentration part of the TDFRS diffraction signal. 


\title{
Mixtures of spherical
} molecules: experiment and RNEMD simulations

\begin{abstract}
We report thermal diffusion forced Rayleigh scattering (TDFRS) measurements on binary mixtures of carbon tetrabromide $\left(\mathrm{CBr}_{4}\right)$, tetraethylsilane and di-tertbutylsilane in carbon tetrachloride $\left(\mathrm{CCl}_{4}\right)$ at different temperatures and concentrations. The Soret coefficient of $\mathrm{CBr}_{4}$ in $\mathrm{CCl}_{4}$ is positive and $\mathrm{S}_{\mathrm{T}}$ of both silanes in $\mathrm{CCl}_{4}$ is negative, which implies that always the heavier component moves to the cold side. This is the expected behavior for unpolar simple molecules. Both silanes have the same mass so we could study the influence of the difference in shape and moment of inertia. For all three systems, $S_{\mathrm{T}}$ decreases with decreasing $\mathrm{CCl}_{4}$ concentration. The results are discussed in the framework of thermodynamic theories and the Hildebrand parameter concept. Additionally, we determined the Soret coefficients for both silane/CCl $\mathrm{Cl}_{4}$ systems by non-equilibrium molecular-dynamics calculations. The simulations predict the correct direction of the thermophoretic motion and reflect the stronger drive towards the warm side for di-tert-butylsilane compared to the more symmetric tetraethylsilane. The values deviate systematically between 9-18\% from the experimental values. *
\end{abstract}

\subsection{Introduction}

Thermal diffusion describes the migration of molecules in a temperature gradient. The molecular origin of the effect, also called Ludwig-Soret effect, is one of the unsolved problems in

* The work described in this chapter is based on J. Chem. Phys. 127, 014502, 2007 
physical chemistry. In some cases, even qualitative predictions, which are of practical importance, are impossible. The main practical applications are separation processes [145, 29] such as thermal field flow fractionation of polymers and colloids or isotope separation, characterization of geochemical processes [66, 30] and combustion [128].

Thermal diffusion in liquid mixtures of non-polar fluids is known to reflect a range of microscopic properties such as the mass, size, and shape of the molecules as well as their interactions [179]. In mixtures of polar liquids, specific interactions between the molecules dominate the thermal diffusion process, while mass and size of the molecules are most important in Lennard-Jones fluids. For liquids more complex than Lennard-Jones fluids, the Soret effect appears to depend on a delicate balance of the molecular properties of the components $[36,183]$.

Even for fairly simple solvents without specific interactions, there is often no simple relation between the Soret coefficient and other physical properties. Especially, if the solvent molecules are not approximately spherical, deviations from some simple rules of thumb are found. Those are that typically the component with the larger mass or higher density moves to the cold side, and that the effect becomes stronger if the components are less miscible [179]. For isotopic mixtures of benzene and cyclohexane, it was found that the Soret coefficient can be written as a sum of three contributions: the mass difference, the difference in moment of inertia and a chemical contribution [183]. Recently, it was shown by equilibrium and nonequilibrium simulations of Lennard-Jones mixtures that the composition dependence of the Soret effect is determined by the chemical contribution [9].

The values of the Soret coefficient can also be compared with the cohesive energy density, also referred to as the Hildebrand solubility parameter [37, 126, 94, 73]. Two substances are mutually soluble if the free energy of mixing $\Delta G_{M}$ is negative. By definition $\Delta G_{M}$ is given by

$$
\Delta G_{M}=\Delta H_{M}-T \Delta S_{M}
$$

where $\Delta H_{M}$ is the enthalpy of mixing per unit volume and $\Delta S_{M}$ is the entropy of mixing per unit volume. The value of $\Delta S_{M}$ is determined by the properties of the given mixture such as composition, compressibility and specific interactions (e.g. hydrogen bonds). For mixtures of nearly spherical molecules without specific interactions and almost the same sizes, the value of $\Delta S_{M}$ is always positive, while there is a certain limiting positive value of $\Delta H_{M}$ above which 
dissolution is impossible. Hildebrand first correlated solubility with the cohesive properties of the solvents and made the ansatz for a binary mixture

$$
\Delta H_{M}=\varphi_{1} \varphi_{2}\left(\delta_{1}^{2}-\delta_{2}^{2}\right)
$$

where $\varphi_{1}$ and $\varphi_{2}$ are volume fractions and $\delta_{1}$ and $\delta_{2}$ are the solubility parameters of the two components. Eq. 2.2 predicts that $\Delta H_{M}=0$ if $\delta_{1}=\delta_{2}$. Hence, two substances with equal solubility parameters should be mutually soluble. In this limit, the two components become identical to each other, what makes the Soret coefficient of such mixture equal to zero. This hypothesis was confirmed by simulations of $S_{\mathrm{T}}$ for binary LJ mixtures [126]. It was found that the component with the larger Hildebrand parameter moves to the cold side. Later, this concept was successfully applied to non-LJ mixtures of components, which still can be mapped onto LJ spheres [179].

Thermodiffusion in Lennard-Jones binary fluids was investigated by molecular dynamic (MD) simulations $[126,56]$. In those simulations, the influence of the different LJ-parameters mass, atomic diameter and interaction strength has been investigated systematically for binary mixtures. The ratio of one of the parameters was varied, while the others were fixed and equal for both compounds. The magnitude of the Soret coefficient was observed to depend on all three ratios. It was found that the heavier species, the smaller species and species with higher interaction strengths tend to accumulate in the cold region.

In this chapter we investigate rather simple tetrahedral, non-polar molecules, which can be well approximated by a spherical shape. Experiments on mixtures of tetrahedral liquids are in principle possible; the side atoms can be halogens, hydrogen or alkyl groups $\left(\mathrm{CH}_{3}, \mathrm{C}_{2} \mathrm{H}_{5}\right)$, whereas the central atom can be turned into $\mathrm{C}, \mathrm{Si}, \mathrm{Ge}, \mathrm{Pb}, \mathrm{Sn}$, Ti or other transition metals. Unfortunately, many of the chemicals containing $\mathrm{Sn}$ and $\mathrm{Pb}$ are highly toxic. And most of the others containing $\mathrm{Sn}, \mathrm{Ge}, \mathrm{Si}$, Ti react with water vapor so that they are not suitable for systematic investigations with our present set-up. After screening many spherical solvents, we picked carbon tetrachloride $\left(\mathrm{CCl}_{4}\right)$, (cf. Fig. 2.1) which has a rather small moment of inertia (cf. Tab. 2.1). Additionally, we selected tetraethylsilane and di-tert-butylsilane, which differ in their moment of inertia, but have the same molar mass, which is slightly lower than the mass of $\mathrm{CCl}_{4}$ (cf. Tab. 2.1). The size of both molecules corresponds roughly to a sphere with van der Waals radius of 3.51 and $3.57 \AA$ respectively (cf. Tab. 2.1). Tetraethylsilane can better be approximated by a sphere than di-tert-butylsilane. As heavier compound we picked 
carbon tetrabromide $\left(\mathrm{CBr}_{4}\right)$, which is solid at room temperature, but dissolves in $\mathrm{CCl}_{4}$. Both carbon tetrahalides can be approximated by spheres with van der Waals radii of 2.91 and 2.73 $\AA ̊$ respectively (cf. Tab. 2.1 and Sec. 2.2).

\section{$\mathrm{CBr}_{4}$}

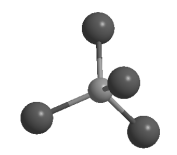

$\mathrm{Si}\left(\mathrm{C}_{2} \mathrm{H}_{5}\right)_{4}$

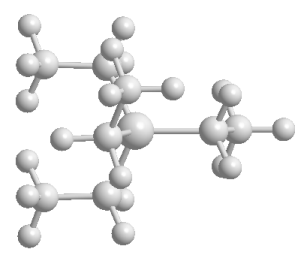

$\mathrm{SiH}_{2}\left[\left(\mathrm{CH}_{3}\right)_{3} \mathrm{C}\right]_{2}$
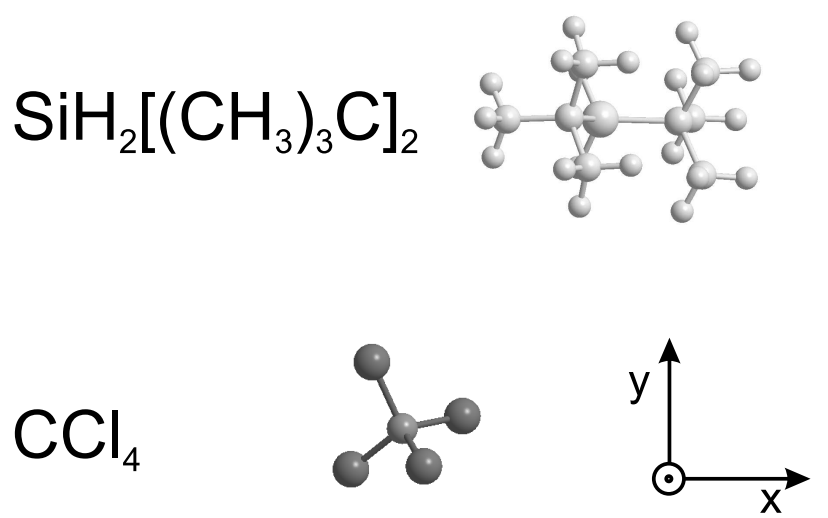

Figure 2.1: Sketch of the investigated molecules carbon tetrabromide $\left(\mathrm{CBr}_{4}\right)$, tetraethylsilane, di-tert-butylsilane and carbon tetrachloride $\left(\mathrm{CCl}_{4}\right)$ as ball-and-stick models. The diameter of the silanes is roughly twice as large as the diameter of $\mathrm{CBr}_{4}$ and $\mathrm{CCl}_{4}$. The reference system used for the calculation of the moments of inertia listed in Tab. 2.1 is indicated. The z-axis is perpendicular to the paper plane. 
Table 2.1: Physical properties of the investigated solvents $\left(M_{w}\right.$ : molecular weight, $\rho$ : density, $\mathrm{r}_{w}$ : van der Waals radius, $T_{\text {boil }}$ : boiling temperature, $\delta$ : Hildebrand parameter at the boiling point and $\mathrm{I}_{i}$ : the moment-of-inertia components about the symmetry axes (cf. Fig. 2.1)). The values of the main moments of inertia for all investigated compounds were calculated using an atomistic model for a single molecule in vacuum [5].

\begin{tabular}{|l|r|r|r|r|r|r|}
\hline solvent & $\begin{array}{r}M_{w} / \\
\mathrm{g} / \mathrm{mol}\end{array}$ & $\begin{array}{r}\rho / \\
\mathrm{kg} / \mathrm{m}^{3} \\
\text { Ref.[1] }\end{array}$ & $\begin{array}{r}\mathrm{r}_{w} / \\
\AA\end{array}$ & $\begin{array}{r}T_{\text {boil }} / \\
{ }^{\circ} \mathrm{C}\end{array}$ & $\begin{array}{r}\delta \\
\mathrm{MPa}^{1 / 2}\end{array}$ & $\begin{array}{r}I_{x} ; I_{y} ; I_{z} / \\
\mathrm{g}^{2} / \mathrm{mol} \\
\text { Ref.[1] }\end{array}$ \\
\hline $\mathrm{CBr}_{4}$ & 331.6 & solid & 2.91 & 190 & 18.1 & $796 ; 796 ; 796$ \\
$\mathrm{Si}\left(\mathrm{C}_{2} \mathrm{H}_{5}\right)_{4}$ & 144.3 & 0.761 & 3.51 & 153 & 13.4 & $452 ; 492 ; 593$ \\
{$\left[\left(\mathrm{CH}_{3}\right)_{3} \mathrm{C}_{2} \mathrm{SiH}_{2}\right.$} & 144.3 & 0.729 & 3.57 & 129 & 12.7 & $246 ; 637 ; 650$ \\
& & & & & \\
$\mathrm{CCl}_{4}$ & 153.8 & 1.59 & 2.73 & 76 & 17.0 & $282 ; 282 ; 282$ \\
\hline
\end{tabular}

\subsection{Experiment}

\subsubsection{Sample Preparation.}

Carbon tetrachloride (99,8\%) was purchased from Fluka and carbon tetrabromide (99\%), tetraethylsilane (99\%) and di-tert-butylsilane (97\%) were ordered from Aldrich. Carbon tetrabromide $\left(\mathrm{CBr}_{4}\right)$ forms white crystals at room temperature. The mole fraction was adjusted by the molecular mass and weight fraction of the components. At higher mole fractions $(x \gtrsim 0.5), \mathrm{CBr}_{4}$ becomes insoluble in $\mathrm{CCl}_{4}$. For the highest mole fraction investigated $(x=0.45), \mathrm{CBr}_{4}$ remains dissolved in $\mathrm{CCl}_{4}$ for at least one day. All samples contained a small amount of Quinizarin (Aldrich). The weight fraction of the dye is approximately 0.002 wt\% corresponding to the optical density of 1.5 for a $1 \mathrm{~cm}$ cell. This amount ensures a sufficient temperature modulation of the optical grating. On the other hand, the quantity is small enough to avoid convection and contributions of the dye to the concentration signal. Approximately $2 \mathrm{ml}$ of the freshly prepared solution were filtered through $0.2 \mu \mathrm{m}$ filter (hydrophobic PTFE) into an optical quartz cell with $0.2 \mathrm{~mm}$ optical path length (Helma), which had been carefully cleaned from dust particles before usage. Experiments were performed in 
a temperature range from 20 to $40^{\circ} \mathrm{C}$.

Properties and Physical Parameters. Table 2.1 lists the properties of the substances, such as molecular weight $M_{w}$, density $\rho$, van der Waals radius $r_{w}$, boiling temperature $T_{\mathrm{boil}}$, the Hildebrand parameter $\delta$ at the boiling point and the moments of inertia about the symmetry axes $\mathrm{I}_{i}$. We estimated $\delta$ by

$$
\delta=\left(\frac{\Delta H_{v a p}-R T}{V}\right)^{\frac{1}{2}},
$$

where $\Delta H_{\text {vap }}$ is the enthalpy of vaporization and $V$ is the molar volume of the pure component. The enthalpy of vaporization at the boiling point was estimated using Trouton's rule. Trouton's rule states that the entropy change for vaporization is approximately $88 \mathrm{Jmol}^{-1} \mathrm{~K}^{-1}$ and holds for many liquids to within $10 \%$ [166].

$$
\Delta H_{\text {vap }}=\Delta S_{\text {vap }} T_{\text {boil }}
$$

The obtained values (cf. Tab. 2.1) compare well with the literature values at lower temperatures (13.9 $\mathrm{MPa}^{1 / 2}$ [28] for tetraethylsilane and $17.3 \mathrm{MPa}^{1 / 2}$ [186] for carbon tetrachloride, estimated from the enthalpy of vaporization at 298K). For carbon tetrabromide we found only a value of $20.1 \mathrm{MPa}^{1 / 2}[28,2] 70 \mathrm{~K}$ below the boiling temperature. The fact that these three experimental values are slightly higher is consistent with the fact, that the Hildebrand parameter increases with decreasing temperature.

Van der Waals radii for all substances were estimated using the van der Waals increment method [47] (cf. Tab. 2.1). This method gives the same value of $3.48 \AA$ for both tetraehylsilane and di-tert-butylsilane. Estimation from the liquid volume $[47,186]$ for these two compounds gives $3.57 \AA$ and $3.66 \AA$ respectively. The effective hard-sphere diameter of tetraethylsilane calculated from modified van der Waals equation of state is equal to $3.49 \AA$ [187] which agrees with our estimates. The averaged values of van der Waals radii for the silanes are listed in Tab. 2.1.

\subsubsection{Refractive index increment measurements.}

Refractive index increments with concentration $(\partial n / \partial c)_{p, T}$ at a constant pressure and temperature were measured using an Abbe refractometer. The temperature derivatives of the 


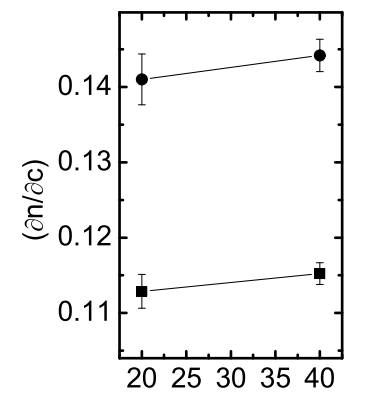

(a) temperature $/{ }^{\circ} \mathrm{C}$

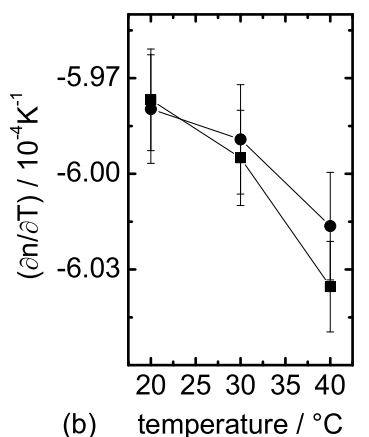

Figure 2.2: Refractive index increments $(\partial n / \partial c)_{p, T}$ and $(\partial n / \partial T)_{p, c}$ of $\mathrm{CBr}_{4}$ in $\mathrm{CCl}_{4}$ as a function of temperature. The mole fractions of $\mathrm{CBr}_{4}$ are: $0.1(\boldsymbol{\square})$ and $0.25(\bullet)$.

refractive index $(\partial n / \partial T)_{p, c}$ at a constant pressure and concentration were determined in temperature range $T \pm 3{ }^{\circ} \mathrm{C}$ using a Michelson interferometer [14].

Figure 2.2 shows both refractive index increments at different temperatures and concentrations for $\mathrm{CBr}_{4}$ in $\mathrm{CCl}_{4} .(\partial n / \partial c)_{p, T}$ increases, while $(\partial n / \partial T)_{p, c}$ decreases with increasing temperature. The values for the higher concentration of $\mathrm{CBr}_{4}$ in $\mathrm{CCl}_{4}$ is slightly higher. The error bars typically around $0.3 \%$, represent one standard deviation between repeated measurements. The refractive index increment with temperature is almost insensitive to the concentration. Figures 2.3 and 2.4 show refractive index increments at different temperatures and concentrations for $\mathrm{Si}\left(\mathrm{C}_{2} \mathrm{H}_{5}\right)_{4}$ and $\left[\left(\mathrm{CH}_{3}\right)_{3} \mathrm{C}\right]_{2} \mathrm{SiH}_{2}$ in $\mathrm{CCl}_{4}$.

\subsubsection{TDFRS experiment and data analysis}

The principle of the TDFRS method is described elsewhere in details [113]. An argon-ion laser $\left(\lambda_{w}=488 \mathrm{~nm}\right)$ is used for writing the grating. The laser beam is split into two writing beams of equal intensity by a beam splitter. An intensity grating is created in the sample by the interference of two laser beams. A small amount of dye in the sample converts the intensity grating into a temperature grating, which in turn causes a concentration grating by the effect of thermal diffusion. Both gratings contribute to a combined refractive index 


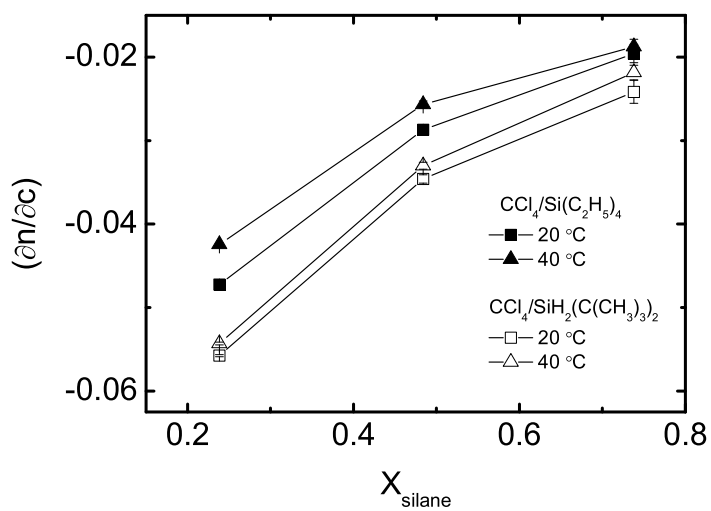

Figure 2.3: Refractive index increments $(\partial n / \partial c)_{p, T}$ of tetraethylsilane (solid symbols) and di-tert-butylsilane (open symbols) in carbon tetrachloride as a function of concentration.

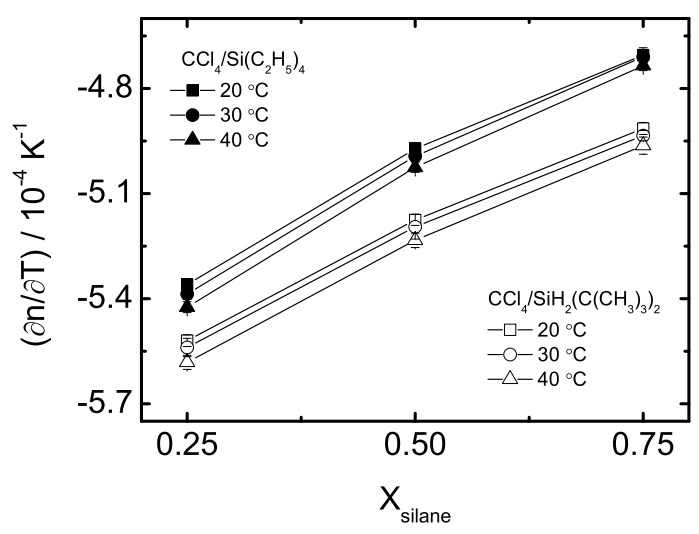

Figure 2.4: Refractive index increments $(\partial n / \partial T)_{p, c}$ of tetraethylsilane (solid symbols) and di-tert-butylsilane (open symbols) in carbon tetrachloride as a function of concentration. 
grating, which is read out by diffraction of a third laser beam $\left(\lambda_{r}=633 \mathrm{~nm}\right)$. The heterodyne signal intensity of the read-out laser is proportional to the amplitude of the refractive index difference $\Delta n(T, c)$ as

$$
\Delta n(T, c)=\left(\frac{\partial n}{\partial T}\right)_{p, c} \Delta T+\left(\frac{\partial n}{\partial c}\right)_{p, T} \Delta c .
$$

where $\Delta T$ and $\Delta c$ are the difference in temperature and concentration, respectively.

The total intensity $\zeta_{\text {het }}(t)$ normalized to the thermal signal is related to the Soret coefficient as

$$
\zeta_{\text {het }}(t)=1-\left(\frac{\partial n}{\partial T}\right)_{p, c}^{-1}\left(\frac{\partial n}{\partial c}\right)_{p, T} S_{\mathrm{T}} c(1-c)\left(1-e^{-q^{2} D t}\right) .
$$

where $q$ is the grating vector and $D$ is the mutual diffusion coefficient.

To determine the transport coefficients, Eq. 2.6 is fitted to the measured heterodyne signal (Fig. 2.5) using contrast factors $(\partial n / \partial c)_{p, T}$ and $(\partial n / \partial T)_{p, c}$ which are measured separately. The residuals are small (less than $1 \%$ ) and show no systematic deviations (Fig. 2.5).

\subsection{Results}

Fig. 2.6 shows the concentration dependence of the transport coefficients for the mixture of $\mathrm{CBr}_{4}$ and $\mathrm{CCl}_{4}$. The mole fractions of $\mathrm{CBr}_{4}$ are 0.1 and 0.25 . The refractive index of this system increases with increasing $\mathrm{CBr}_{4}$ content, so that $(\partial n / \partial c)_{p, T}$ is positive (cf. Fig. 2.2) and the refractive index increment with temperature $(\partial n / \partial T)_{p, C}$ is negative (cf. Fig. 2.2). The concentration part of the TDFRS-signal increases exponentially (Fig. 2.5), which implies that the Soret coefficient is positive and the heavier component $\mathrm{CBr}_{4}$ migrates to the cold side. The diffusion coefficient increases with increasing temperature and decreases with increasing $\mathrm{CBr}_{4}$ content. This behavior can be explained by a lower viscosity at higher temperatures and higher $\mathrm{CBr}_{4}$ contents.

In Fig. 2.7 and 2.8 the concentration dependence of $S_{\mathrm{T}}, D$ and $D_{\mathrm{T}}$ for tetraethysilane and di-tert-butylsilane is displayed. The Soret coefficient of both silanes is negative, which implies that the lighter silanes move to the warm side, while the heavier and denser $\mathrm{CCl}_{4}$ migrates to the cold. As in the case of $\mathrm{CBr}_{4}$, the rule of thumb that typically the heavier compound moves to the cold side is obeyed. The magnitude of the Soret coefficient for ditert-butylsilane is roughly $10 \%$ larger than the one of tetraethylsilane. Both systems show 


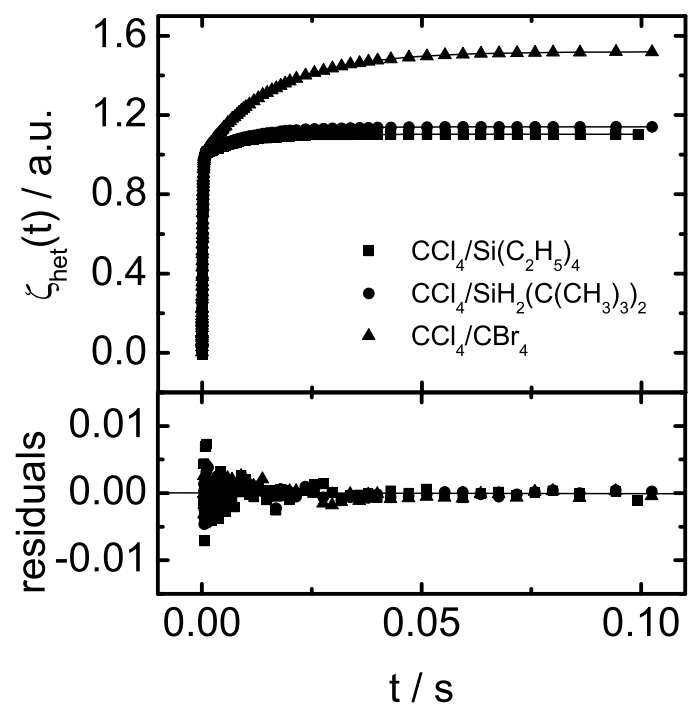

Figure 2.5: Typical normalized TDFRS signals and the residuals plot to the fitting curve according to Eq. 2.7 of investigated mixtures at a temperature of $20^{\circ} \mathrm{C}$. Mole fraction of $\mathrm{CCl}_{4}$ equals to 0.75 . The solid lines refer to a fit according to Eq. 2.6. 


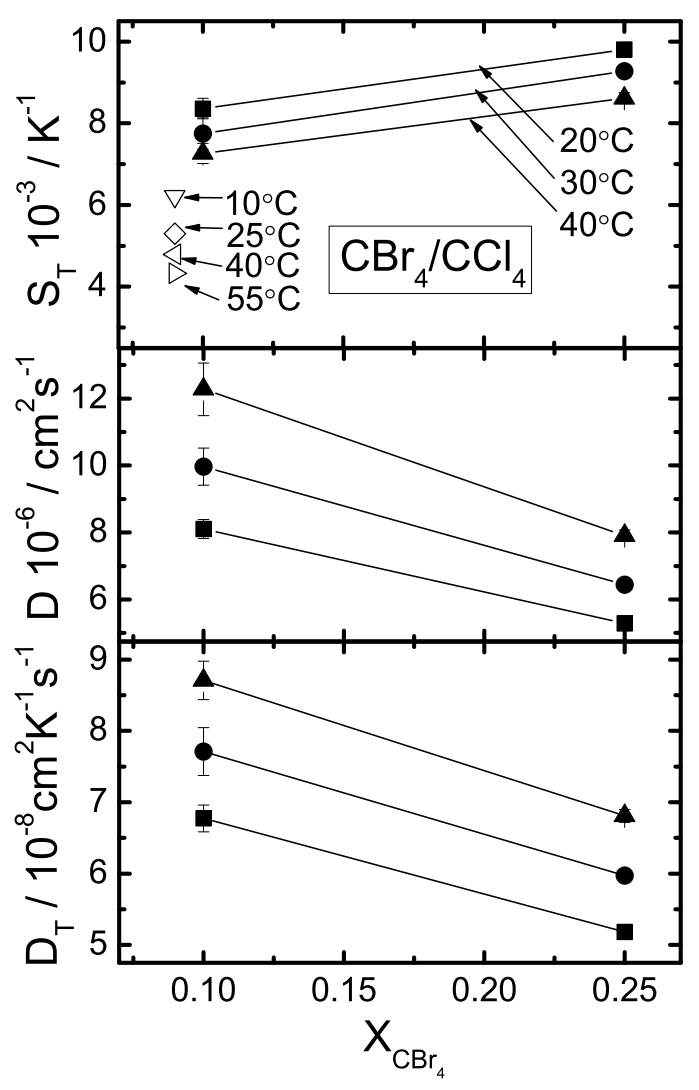

Figure 2.6: Soret coefficient $S_{\mathrm{T}}$, diffusion coefficient $D$ and thermal diffusion coefficient $D_{\mathrm{T}}$ of $\mathrm{CBr}_{4}$ in $\mathrm{CCl}_{4}$ as a function of concentration. Solid symbols - our data, open symbols - data from Saxton et al. [144]. 


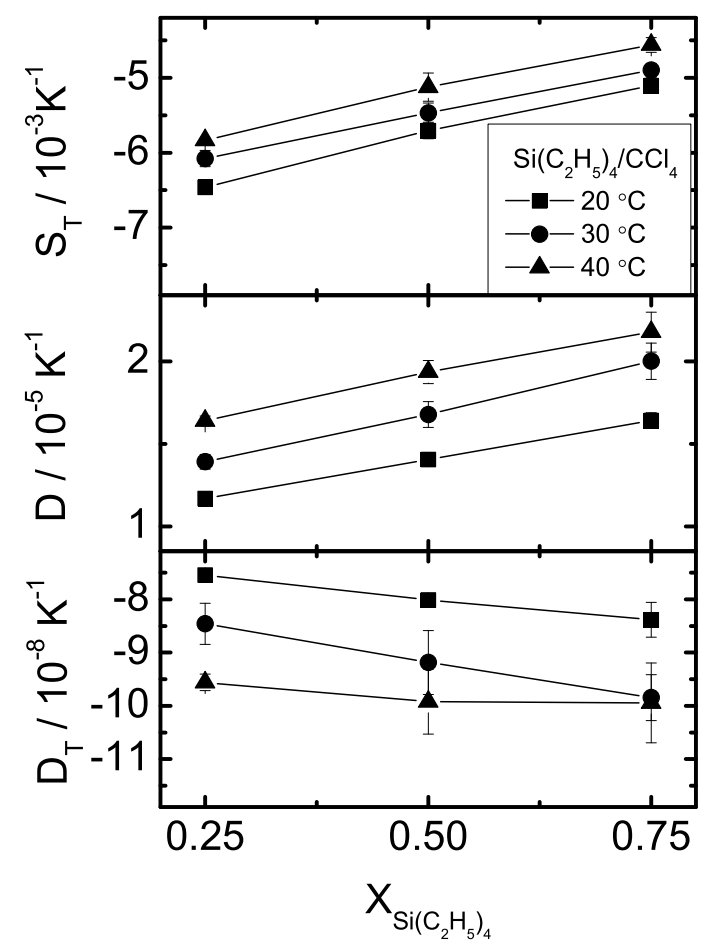

Figure 2.7: Soret coefficient $S_{\mathrm{T}}$, mutual diffusion coefficient $D$ and thermal diffusion coeffi-

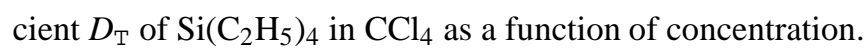

a weak concentration dependence, which is typical for unpolar substances [75, 113]. As expected, the diffusion coefficient increases with increasing temperature and with increasing amount of the less viscous silane. For all systems investigated, an increasing concentration of the heavier component leads to a decreasing diffusion coefficient.

\subsection{Simulations}

The reverse nonequilibrium molecular dynamics method has been applied to investigate the thermal diffusion of carbon tetrachloride in silanes. In the following, we only briefly summa- 


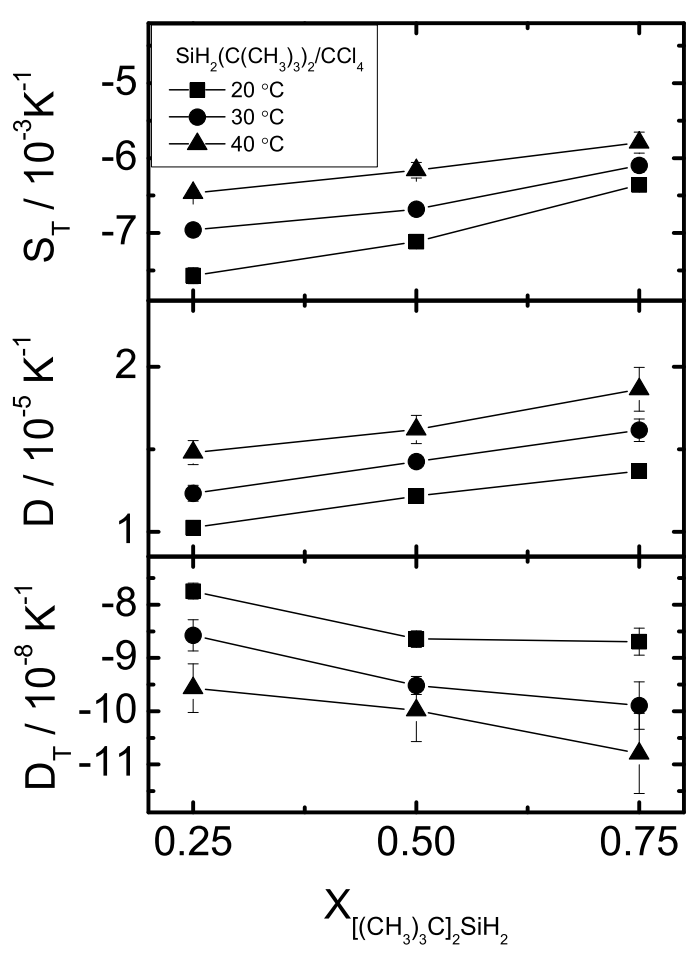

Figure 2.8: Soret coefficient $S_{\mathrm{T}}$, mutual diffusion coefficient $D$ and thermal diffusion coefficient $D_{\mathrm{T}}$ of $\left[\left(\mathrm{CH}_{3}\right)_{3} \mathrm{C}\right]_{2} \mathrm{SiH}_{2}$ in $\mathrm{CCl}_{4}$ as a function of concentration. 
Table 2.2: Lennard-Jones parameters for tetraethylsilane, di-tert-butylsilane and carbon tetrachloride (* - Lennard-Jones parameters were adjusted to get experimental density and heat of vaporization).

\begin{tabular}{|c|c|c|}
\hline atom type & $\begin{array}{c}\sigma / \\
\mathrm{nm}\end{array}$ & $\begin{array}{r}\varepsilon / \\
\mathrm{kJ} / \mathrm{mol}\end{array}$ \\
\hline C (Ref.[127]) & 0.3774 & 0.2277 \\
\hline $\mathrm{Cl}$ (Ref.[127]) & 0.3467 & 1.0945 \\
\hline $\mathrm{Si}^{*}$ & 0.5 & 0.42 \\
\hline $\mathrm{SiH}_{2}^{*}$ & 0.559 & 0.5 \\
\hline $\mathrm{CH}_{2}$ Ref.[103, 177] (for $\left.\mathrm{Si}\left(\mathrm{C}_{2} \mathrm{H}_{5}\right)_{4}\right)$ & 0.393 & 0.3808 \\
\hline $\mathrm{CH}_{3}$ Ref.[103, 177] (for $\left.\mathrm{Si}\left(\mathrm{C}_{2} \mathrm{H}_{5}\right)_{4}\right)$ & 0.391 & 0.8647 \\
\hline C Ref.[103, 177] (for $\left.\left[\left(\mathrm{CH}_{3}\right)_{3} \mathrm{C}\right]_{2} \mathrm{SiH}_{2}\right)$ & 0.391 & 0.1413 \\
\hline $\mathrm{CH}_{3}$ Ref. $[103,177]$ (for $\left[\left(\mathrm{CH}_{3}\right)_{3} \mathrm{C}\right]_{2} \mathrm{SiH}_{2}$ ) & 0.385 & 0.582 \\
\hline
\end{tabular}

rize the force fields used for the liquid solvents and give some simulation details. A detailed description can be found elsewhere [189].

The force field for carbon tetrachloride In obtaining the force field for carbon tetrachloride we followed the work by Rey at al. [127]. They developed a consistent set of molecular models for MD simulations of a whole family of methylchloromethanes $\left(\left(\mathrm{CH}_{3}\right)_{4-n} \mathrm{CCl}_{n}\right)$, providing excellent accordance with thermodynamic properties (liquid density and heat of vaporization). It was found that non-negligible electrostatic effects on the liquid structure exist only for the polar cases $(n=2,3)$. Therefore, we used the proposed force field for carbon tetrachloride without charges (see Tab. 2.2).

The force field for the silanes For tetraethylsilane we used a force field derived by Striolo et al [160]. Atoms in the same molecule interact with each other via short-range potentials that account for bond length, bond angle and torsional potentials. It was shown that by combining force fields independently developed to describe silsesquioxanes (POSS) and alkanes (Tab. 2.2) it is possible to predict correctly the structure of isolated hybrid polyhedral oligomeric silsesquioxane monomers as well as that of crystals composed solely of POSS 
Table 2.3: Thermodynamic results obtained for pure tetraethylsilane, di-tert-butylsilane and carbon tetrachloride compared with the corresponding experimental results at $298 \mathrm{~K}$.

\begin{tabular}{|c|c|c|c|c|}
\hline solvent & $\begin{array}{r}\rho_{\text {exp }} / \\
\mathrm{kg} / \mathrm{m}^{3}\end{array}$ & $\begin{array}{r}\rho_{\text {sim }} / \\
\mathrm{kg} / \mathrm{m}^{3}\end{array}$ & $\begin{array}{c}\Delta H_{\text {vap }}^{\exp } / \\
\mathrm{kJ} / \mathrm{mol}\end{array}$ & $\begin{array}{r}\Delta H_{v a p}^{\text {sim }} \\
\mathrm{kJ} / \mathrm{mol}\end{array}$ \\
\hline $\mathrm{Si}\left(\mathrm{C}_{2} \mathrm{H}_{5}\right)_{4}$ & $\begin{array}{r}0.761 \\
\text { Ref.[1] }\end{array}$ & 0.773 & $\begin{array}{r}39 \\
\text { Ref.[28] }\end{array}$ & 37.9 \\
\hline$\left[\left(\mathrm{CH}_{3}\right)_{3} \mathrm{C}\right]_{2} \mathrm{SiH}_{2}$ & $\begin{array}{r}0.729 \\
\text { Ref.[1] }\end{array}$ & 0.735 & - & 38.1 \\
\hline $\mathrm{CCl}_{4}$ & $\begin{array}{r}1.594 \\
\text { Ref.[1] }\end{array}$ & 1.588 & $\begin{array}{r}31.5 \\
\text { Ref.[186] }\end{array}$ & 30.06 \\
\hline
\end{tabular}

monomers [160].

Test MD runs of a cubic periodic box of 200 tetraethylsilane molecules at $298 \mathrm{~K}$ and $1 \mathrm{~atm}$ yielded a unsatisfactory solvent density $\left(0.795 \mathrm{~kg} / \mathrm{m}^{3}\right)$ and heat of vaporization $(33.2 \mathrm{~kJ} / \mathrm{mol})$ (density $4.3 \%$ too high, heat of vaporization $5.8 \mathrm{~kJ} / \mathrm{mol}$ too low). Experimental values at $298 \mathrm{~K}$ are presented in Tab. 2.3. Another difficulty of this model is that it is only applicable to silanes with linear alkyl chain attached (the alkane backbone was modeled according to the TraPPE united-atom force field [93] developed for the linear alkanes). Slightly different parameters were required for the $\mathrm{CH}_{3}$ groups of branched alkanes in order to reproduce the phase diagram [103, 177]. Therefore, we had to use a different route to describe di-tertbutylsilane, which has branched alkane structures attached.

In order to simulate both silanes consistently on the basis of the same force field parameters we derived new united atom force field parameters (Tab. 2.2). For the alkane we applied the force field from Nath [103, 177]. In the case of tetraethylsilane, the Lennard Jones parameters $\sigma$ and $\varepsilon$ of $\mathrm{Si}$, and for di-tert-butylsilane those of $\mathrm{SiH}_{2}$ have been adjusted. These parameters were increased to reproduce the experimental density and the heat of vaporization for the systems (Tab. 2.2 and 2.3).

Computational details Two binary equimolar mixtures of carbon tetrachloride with tetraethylsilane and di-tert-butylsilane were simulated at $T=303 \mathrm{~K}$ and $P=1$ atm. The YASP package 


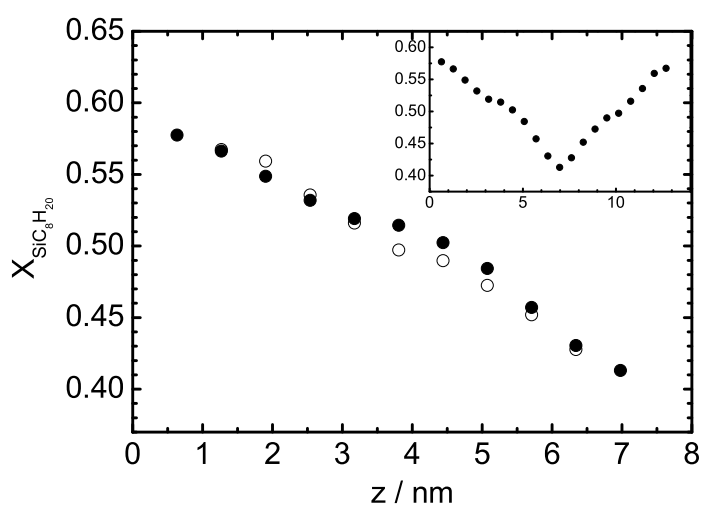

Figure 2.9: Concentration profiles for a equimolar mixture of tetraethylsilane/ $\mathrm{CCl}_{4}$ averaged over a simulation time of $52 \mathrm{~ns}$. The inset shows the complete concentration profile. The open symbols represented the upward branch of the full profile, which has been flipped over at the coldest slab.

[101] was used. The cell was elongated in $z$-direction, which is the direction of the heat flow $\left(\mathrm{L}_{x}=\mathrm{L}_{y}=\mathrm{L}_{z} / 3 \approx 4 \mathrm{~nm}\right.$ ). The cutoff length for nonbonded interactions was $1.2 \mathrm{~nm}$. The time step was 2 fs. All reverse noneqilibrium molecular-dynamics (RNEMD) simulations were performed at constant NVT conditions (densities: 1047.2 and $1009.9 \mathrm{~kg} / \mathrm{m}^{3}$ for equimolar mixtures of tetraethylsilane and di-tert-butylsilane in carbon tetrachloride, respectively) with 960 molecules in the simulation box. The average temperature was kept constant by the thermostat of Berendsen et al. [16], with the temperature coupling time being $\mathrm{T}=1 \mathrm{ps}$. Figures 2.9 and 2.10 show the concentration profiles for equimolar mixtures of tetraethylsilane and di-tert-butylsilane in $\mathrm{CCl}_{4}$ averaged over a simulation time of 51.9 and $74.12 \mathrm{~ns}$, respectively. The time development of the Soret coefficient for both mixtures is shown in Figure 2.11 as cumulative average. For each silane, two values are displayed. One has been calculated from 9 slabs of the downward branch and another from 9 slabs in the upward branch. The hottest and coldest slabs have been excluded from the analysis. After $40 \mathrm{~ns}$, the $S_{\mathrm{T}}$-value for tetraethylsilane/ $\mathrm{CCl}_{4}$ converges to a plateau value of $-5 \times 10^{-3} \mathrm{~K}^{-1}$. In the case of the system di-tert-butylsilane/ $/ \mathrm{CCl}_{4}$ the plateau $\left(-5.5 \times 10^{-3} \mathrm{~K}^{-1}\right)$ is reached later 


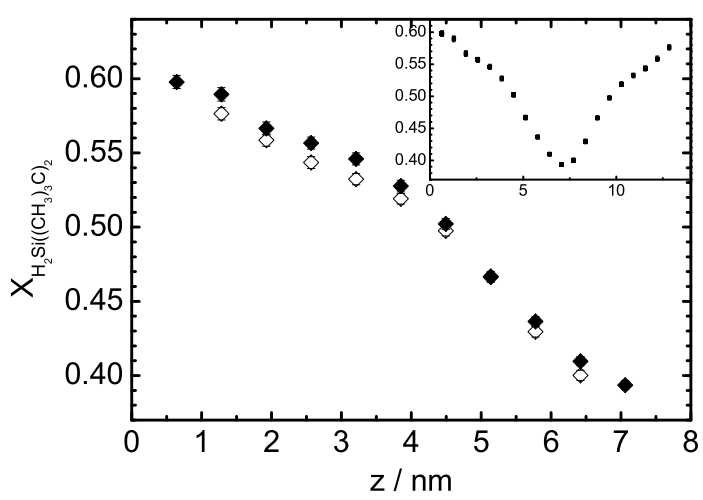

Figure 2.10: Concentration profiles for a equimolar mixture of di-tert-butylsilane/ $\mathrm{CCl}_{4}$ averaged over a simulation time of $55 \mathrm{~ns}$. The inset shows the complete concentration profile. The open symbols represented the upward branch of the full profile, which has been flipped over at the coldest slab.

after $60 \mathrm{~ns}$. The average values $S_{\mathrm{T}}=-5.5 \times 10^{-3} \mathrm{~K}^{-1}$ for di-tert-butylsilane in $\mathrm{CCl}_{4}$ is $10 \%$ smaller than the value obtained for tetraethylsilane in $\mathrm{CCl}_{4}$. The difference is not very pronounced, but this tendency is also confirmed in the experiment, although the magnitude of the experimental values is systematically $9-18 \%$ larger.

\subsection{Discussion}

\subsubsection{Comparison of the experimental and simulation results}

The following values for the Soret coefficient were determined by simulations at around $303 \mathrm{~K}$ for a binary equimolar mixtures of tetraethylsilane and di-tert-butylsilane in carbon tetrachloride: $-5 \pm 0.3 \times 10^{-3}$ and $-5.5 \pm 0.3 \times 10^{-3} \mathrm{~K}^{-1}$. The simulation results are larger than the experimental values and their magnitude is smaller by 9 and $18 \%$. Those small systematic errors are probably due to the force field parameters, which were developed to reproduce the density and heat of vaporization of the pure components but not any transport 


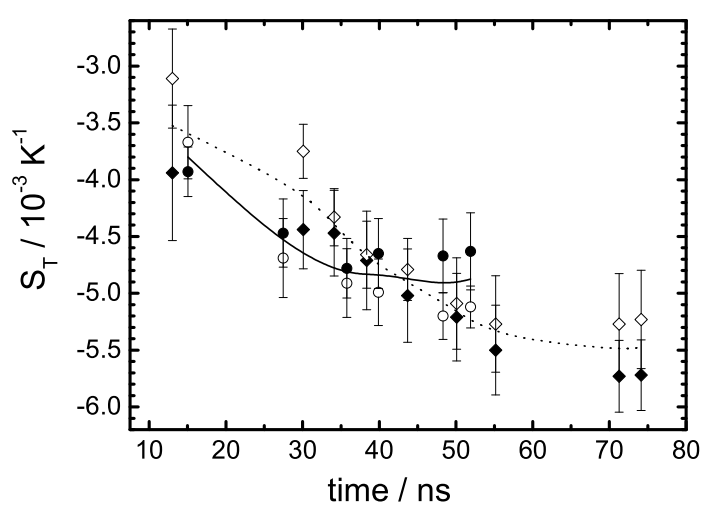

Figure 2.11: Soret coefficients of the tetraethylsilane (circles) and di-tert-butylsilane (diamonds) in carbon tetrachloride as a function of simulation time. One set of values has been calculated from the downward (solid symbols) branch and the other values have been calculated from the upward branch (open symbol). The lines are guides to the eye for tetraethylsilane (solid line) and di-tert-butylsilane (dotted line).

coefficient. For gaseous systems it is known that the calculation of the Soret coefficient is much more sensitive to the correct choice of the interaction potential than other properties such as viscosity or diffusion coefficient [174]. Another reason might be the sensitivity of the Soret coefficient to the chosen mixing rule. Galliero et al. [56] showed for methane/n-decane a strong dependence of $S_{\mathrm{T}}$ on the cross interaction parameters $\mathrm{k}_{i, j}$ and $\mathrm{l}_{i, j}$, which determine the interaction strengths $\varepsilon_{1,2}$.

$$
\varepsilon_{1,2}=\left(1-k_{i, j}\right)\left(\varepsilon_{1} \varepsilon_{2}\right)^{0.5}, \sigma_{1,2}=\left(1-l_{i, j}\right) \frac{1}{2}\left(\sigma_{1}+\sigma_{2}\right)
$$

with $\varepsilon_{1,2}, \varepsilon_{1}, \varepsilon_{2}$ the interaction strength parameters and $\sigma_{1,2}, \sigma_{1}, \sigma_{2}$ the diameters.

In our case the classic Lorenz-Berthelot mixing rule $\left(\mathrm{k}_{i, j}=0 ; 1_{i, j}=0\right)$ was used, which had been applied in the simulation of benzene/cyclohexane [189] mixtures. Unfortunately, a validation of the cross interaction parameters was impossible, because to our best knowledge there are no literature data available for the investigated mixtures. In view of other MD simulations of the Soret coefficient of fluid mixtures, some of which use much more sophisticated 
force fields [189], the agreement with experiment must be viewed as very satisfactory.

\subsubsection{Contributions to the Soret coefficient.}

Galliero et al [56] investigated the thermodiffusion behavior of equimolar mixtures of "super methane" in methane. The parameters mass, $m$, diameter, $\sigma$, and depth of the interaction potential, $\varepsilon$ of super methane were different from those of methane. As already done earlier by Reith et al. [126] they varied systematically the ratio of one of the parameters (e.g. $\mathrm{m}_{1} / \mathrm{m}_{2}$ ) while keeping the two other parameters fixed and equal. By this procedure the obtained three additive contributions $\alpha_{T}^{m}, \alpha_{T}^{\sigma}$ and $\alpha_{T}^{\varepsilon}$ of the total thermal diffusion ratio $\alpha_{T}=S_{\mathrm{T}} T$ stemming from the mass, diameter and interaction strength, respectively.

$$
\alpha_{T}=\alpha_{T}^{m}+\alpha_{T}^{\sigma}+\alpha_{T}^{\varepsilon}
$$

This empirical formula reproduces the simulation results quite well, if the ratios deviate not too much from 1 .

We applied equation 2.8 to the investigated mixtures of tetraethylsilane, di-tert-butylsilane and carbon tetrabromide in carbon tetrachloride (component 2). The ratios of the diameter and the depths of the interaction potential were estimated using the experimental molar volumes $\mathrm{V}_{\text {mol }}$ (at room temperature) and enthalpies of vaporization $\mathrm{H}_{v a p}$ (at boiling point) for the different components [125].

$$
\begin{aligned}
& \frac{\sigma_{1}}{\sigma_{2}}=\left(\frac{V_{m o l, 1}}{V_{m o l, 2}}\right)^{\frac{1}{3}} . \\
& \frac{\varepsilon_{1}}{\varepsilon_{2}}=\left(\frac{\Delta H_{\text {vap }, 1}}{\Delta H_{\text {vap }, 2}}\right) .
\end{aligned}
$$

The results are summarized in Tab. 2.4. The mass contribution for $\mathrm{CBr}_{4} / \mathrm{CCl}_{4}$ mixture is positive while for tetraethylsilane/ $\mathrm{CCl}_{4}$ and di-tert-butylsilane/ $\mathrm{CCl}_{4}$ it is negative. This implies that the component with the higher molar mass moves to the cold side. The mass contributions for both tetraethylsilane/ $\mathrm{CCl}_{4}$ and di-tert-butylsilane/ $\mathrm{CCl}_{4}$ are the same. At the same time the difference in size and even more pronounced the difference in the interaction potential leads to a stronger drive of di-tert-butylsilane to the hot side. The values obtained from Eq. 2.8, 2.9 and 2.10 reproduce the correct direction of thermodiffusion motion for all three mixtures, but they are one order of magnitude too small. 
Table 2.4: The values of the Soret coefficient calculated using Eq. 2.8 at 303K. The deviations from the experimental values (equimolar mixtures) are shown in brackets.

\begin{tabular}{|c|c|c|c|c|c|}
\hline solute & $\begin{array}{r}S_{\mathrm{T}}^{m} / \\
10^{-3} \mathrm{~K}^{-1}\end{array}$ & $\begin{array}{r}S_{\mathrm{T}}^{\sigma} / \\
10^{-3} \mathrm{~K}^{-1}\end{array}$ & $\begin{array}{r}S_{\mathrm{T}}^{\varepsilon} / \\
10^{-3} \mathrm{~K}^{-1}\end{array}$ & $\begin{array}{c}S_{\mathrm{T}} \text { from Eq. } 2.8 / \\
10^{-3} \mathrm{~K}^{-1}\end{array}$ & $\begin{array}{r}\text { measured } S_{\mathrm{T}} / \\
10^{-3} \mathrm{~K}^{-1}\end{array}$ \\
\hline $\mathrm{Si}\left(\mathrm{C}_{2} \mathrm{H}_{5}\right)_{4}$ & -0.15 & -0.33 & 0.382 & $\begin{array}{l}-0.11 \\
(98 \%)\end{array}$ & -5.5 \\
\hline$\left[\left(\mathrm{CH}_{3}\right)_{3} \mathrm{C}\right]_{2} \mathrm{SiH}_{2}$ & -0.15 & -0.36 & 0.26 & $\begin{array}{l}-0.25 \\
(96 \%)\end{array}$ & -6.7 \\
\hline $\mathrm{CBr}_{4}$ & 1.77 & -0.07 & 0.57 & 2.26 & $\sim 12$ \\
\hline
\end{tabular}

The values of the Soret coefficient can also be compared with a parameter of the cohesive energy density [37, 126, 94, 73], which is also referred as the Hildebrand solubility parameter. As it is expected in our case the component with the larger Hildebrand parameter (cf. Tab. 2.1) carbon tetrabromide (mixed with carbon tetrachloride) and carbon tetrachloride (mixed with one of the silanes) accumulates in the cold region.

\subsubsection{Discussion of the effect of the moment of inertia.}

The importance of the moments of inertia in the thermal diffusion behavior of the benzene/cyclohexane mixture was reported by Debuschewitz et al. [36]. They found that the component with the larger $\mathrm{I}_{z}$ (the main moment of inertia perpendicular to the plane of the molecule) moves to the cold side. The physical reason of this phenomenon in liquids is not clear. In an old work by Schirdewahn et al. [147] about gaseous mixtures, the moment of inertia part is vaguely related to the rotational diffusion contribution to the thermal diffusion. At the same time, it is obvious that the values of the main moments of inertia for a given molecule characterize its size (or van-der-Waals volume) and the mass distribution, while all three possible ratios $\left(I_{x} / I_{y} I_{x} / I_{z} I_{y} / I_{z}\right)$ characterize the shape. All these factors are responsible for the diffusion (as well as the thermodiffusion) behavior. From this point, one can assume that the value of $\mathrm{I}_{z}$ in case of benzene/cyclohexane mixtures is responsible not only for the size but also for the mass distribution in the molecule but not for the shape. They 
assumed, that both molecules have a disk-like structure, which is not changed by isotopic substitution. In our case carbon tetrachloride, carbon tetrabromide and tetraethylsilane have spherical structures as far as all diagonal elements of their tensors of the moment of inertia are the same (cf. Tab. 2.1). The lower value of $I_{x}$ in comparison to the values of $I_{y}$ and $I_{z}$ for di-tert-butylsilane describe the ellipsoidal shape of the molecule. In order to take into account the shape effect, a more complicated expression for $S_{\mathrm{T}}{ }^{I}$, involving $\mathrm{I}_{x}, \mathrm{I}_{y}$ and $\mathrm{I}_{z}$ for binary mixtures of equivolume components is required. However, the differences in $S_{\mathrm{T}}$ between tetraethylsilane and di-tert-butylsilane are too small to be unambiguously attributed to differences in the moment of inertia. Other effect, such as the anisotropic friction, could also explain an effect of this magnitude.

\subsection{Conclusion}

We investigated the thermal diffusion behavior of three simple mixtures consisting of spherical molecules experimentally by the so called TDFRS method. Additionally we applied a molecular exchange version of the reverse non-equilibrium molecular dynamics algorithm to determine the Soret coefficients for two of the experimentally investigated mixtures.

Contrary to more complex fluids, the observed thermophoretic motion for those three unpolar mixtures follows the common rules, which state that the component with the larger molar mass and the larger Hildebrandt parameter accumulates in the cold region.

We found a fairly good agreement between the simulated and experimentally determined Soret coefficients for the systems tetraethylsilane and di-tert-butylsilane in carbon tetrachloride. Although the magnitude of the simulated $S_{\mathrm{T}}$-values is systematically by $9-18 \%$ smaller than in experiment, both methods found that di-tert-butylsilane accumulates slightly stronger in the warm region than the more symmetric tetraethylsilane. Both investigated silane compounds do have the same molar mass, so that we tried to reveal the moment of inertia contribution to the thermophoretic motion. Here it turned out that the analysis is not so simple because two of the three moments of inertia are changed simultaneously. This requires an expression which connects the Soret coefficient with more than one moment of inertia. In order to obtain such a relation more systematic experiments and simulations need to be performed in the future. In addition, the difference between the two silanes is very small. 


\title{
Mixtures of linear alkanes: A comparison between $\mathrm{TC}$ and TDFRS methods
}

\begin{abstract}
In the present chapter we studied the thermal diffusion behavior of $n$-decane in various alkanes by thermogravitational column (TC) techniques and thermal diffusion forced Rayleigh scattering (TDFRS) method. The investigated lighter alkanes compared to $n$-decane are $n$-pentane, $n$-hexane, $n$-heptane, $n$-octane and the heavier ones are $n$-tetradecane, $n$-pentadecane, $n$-hexadecane, $n$-heptadecane, $n$-octadecane and $n$-eicosane. The binary mixture $n$-decane/n-pentane we investigated at several different concentrations all other mixtures were only investigated at a mass fraction of 50\%. Even for the volatile $n$-pentane/ $n$-decane mixture the deviations between the thermal diffusions coefficients determined by the different methods agreed within the error bars. Typically the agreement between the two methods was in the order of 5\%. Compared to recently published TC and TDFRS data we found deviations in the order of 30 up to $40 \%$. We analyze and discuss the possible reasons for the discrepancies for the present and the past publications. *
\end{abstract}

\subsection{Introduction}

The coupling between a temperature gradient and a resulting mass flux is denoted thermal diffusion or Ludwig-Soret effect in accordance with its discover and its first investigator. Particularly, the investigation of crude oil components such as alkanes and organic ring compounds

* The work described in this chapter is based on J. Phys. Chem. B, 112, 83408345, 2008 
is of practical relevance, because the determination of reliable model parameters is essential for the characterization of geological fields $[30,57,58]$. Additionally the effect plays also an important role in separation techniques for liquid mixtures (see e.g. Refs. [77, 118, 179]).

In the simple case of a binary mixture with constant pressure there is a mass diffusion current $j_{\mathrm{D}}=-\rho D \nabla c$ and a thermal diffusion current $j_{\mathrm{T}}=-\rho D_{\mathrm{T}} c(1-c) \nabla T$, with $c$ the mass fraction, $\rho$ the density of the liquid, and $D$ and $D_{\mathrm{T}}$ the mutual mass and thermal diffusion coefficients, respectively. In the stationary state the two flows cancel and the resulting concentration gradient is given by

$$
\nabla c=-S_{\mathrm{T}} c(1-c) \nabla T
$$

$S_{\mathrm{T}}=D_{\mathrm{T}} / D$ is the Soret coefficient.

The thermal diffusion of non-polar fluid mixtures is sometimes governed by the mass, size, and shape of the molecules as well as their interactions (see Ref. [179] for a review). The influence of the physical parameters on the thermal diffusion behavior has been systematically investigated for isotopic mixtures of benzene and cyclohexane [36, 183]. For these mixtures it was found that the Soret coefficient depends on the mass and moment of inertia difference but also on a chemical contribution. In the case of polar mixtures, specific interactions between the molecules dominate the thermal diffusion process while mass and size are not so important.

A number of studies have focused on the Soret effect in mixtures containing an alkane as one of the components. Different experimental techniques were applied to investigate the thermal diffusion behavior of toluene/hexane [188, 75, 20], alkane/alkane [113, 18, 85], cyclohexane/benzene [36], and $n$-alkane/benzene [81, 80, 37, 46, 165, 20] mixtures. Also in a benchmark study of three binaries one of the components was an alkane [116]. In the past also thermodynamic models [61] have been tested and simulations [113] have been performed for alkane mixtures.

Alkanes belong to the class of non-polar mixtures. Often they are treated as ideal mixtures, because the minor microscopic effects, such as the conformational changes in the molecules have only a very small effect on the usual thermodynamic properties of alkane/alkane mixtures. This tendency is also confirmed in a recent thermal diffusion study of alkane/alkane mixture $[85,18]$, which shows that always the heavier component moves to the cold region. 
We focus in this chapter on the Soret effect in binary alkane/alkane mixtures for which we expect a normal behavior. All mixtures are investigated by thermogravitational column (TC) technique and thermal diffusion forced Rayleigh scattering (TDFRS) method. First we investigate the binary mixture $n$-decane $/ n$-pentane at several different concentration at $27^{\circ} \mathrm{C}$. This mixture we studied even with a cylindrical and parallelepipedic thermogravitational column. The experimental data are compared with earlier experimental data and simulation results [113]. Additionally, we investigated binary mixtures of $n$-decane in $n$-hexane, $n$-heptane, $n$-octane, $n$-tetradecane, $n$-pentadecane, $n$-hexadecane, $n$-heptadecane, $n$-octadecane and $n$ eicosane at a weight fraction of $50 \%$. The obtained experimental data are also compared with recent measurement by the TC method [85].

\subsection{Experiment}

\subsubsection{Sample preparation}

\section{Thermogravitational columns:}

All the products used in the TCs were purchased from Merck with a purity better than $99 \%$. First we always filled in the less volatile component, i.e., the alkane with higher molecular weight; then the corresponding amount of the second alkane is added. The concentrations of the binary mixtures were adjusted by weighting both components separately. The mixtures for the parallelepipedic TC were prepared with a balance with a capacity up to $310 \mathrm{~g}$ and an accuracy of $0.0001 \mathrm{~g}$. For the mixtures of the cylindrical TC we used a balance with a capacity up to $4500 \mathrm{~g}$ and an accuracy of $0.01 \mathrm{~g}$. The sample volume needed to run an experiment in the parallelepipedic and cylindrical TC is approximately $25 \mathrm{~cm}^{3}$ and $300 \mathrm{~cm}^{3}$, respectively. As verification, before and after each experimental run the concentration of the mixture had been determined. The observed concentration change was typically in the order of $\Delta c_{0}=0.0005$.

\section{TDFRS:}

The alkanes $n$-pentane $(\geqslant 99 \%), n$-hexane $(\geqslant 99 \%), n$-heptane $(\geqslant 99.5 \%), n$-octane $(\geqslant 99.5 \%)$, $n$-octadecane $(\geqslant 99 \%)$ and $n$-tetradecane $(\geqslant 99 \%)$ were purchased from Fluka; $n$-decane $(\geqslant 99 \%)$, 
$n$-heptadecane $(99 \%)$ and $n$-eicosane $(99 \%)$ were ordered from Aldrich. The alkane mole fraction of all mixtures was adjusted by weighing the components. The TDFRS experiments require a small amount of dye in the sample. All samples contained approximately 0.002 wt\% of the dye Quinizarin (Aldrich). This amount ensures a sufficient optical modulation of the grating but is small enough to avoid convection and contributions of the dye to the concentration signal. Before each TDFRS experiment, approximately $2 \mathrm{ml}$ of the freshly prepared solution were filtered through $0.2 \mu \mathrm{m}$ filter (hydrophobic PTFE) into an optical quartz cell with $0.2 \mathrm{~mm}$ optical path length (Helma) which was carefully cleaned from dust particles before usage.

After each measurement we checked carefully by monitoring the change of the meniscus height in the two filling capillaries of the sample cell whether the volatile solvent evaporated during the measurement. The accuracy of this method is certainly better than $1 \%$. The total volume of the sample cell is in the order of $0.6 \mathrm{ml}$. Even for the $n$-decane $/ n$-pentane mixture with the lowest pentane content, the concentration change should be less than $\Delta x \approx \Delta c \approx 0.01$.

\subsubsection{Data analysis and set-up}

\section{Thermogravitational columns:}

The TC theory provides a relation between the stationary separation $\Delta c$ and the thermodiffusion coefficient $D_{\mathrm{T}}$. For more details see Ref. [45]:

$$
\Delta c=-\frac{504 L_{z}}{g L_{x}^{4}} \frac{D_{T} v}{\alpha} c_{0}\left(1-c_{0}\right)
$$

Where $\alpha=-(1 / \rho)(\partial \rho / \partial c)$ is the thermal expansion coefficient, $\rho$ the density of the mixture with the initial mass concentration $c_{0}, v$ the kinematic viscosity and $g$ the gravity acceleration. $L_{z}$ is the height of the column, which is $500 \mathrm{~mm}$ for the cylindrical TC and $530 \mathrm{~mm}$ for the parallelepipedic TC. And $L_{x}$ is the gap between the two vertical walls, which is $1.000 \pm$ $0.005 \mathrm{~mm}$ for the cylindrical TC and $1.50 \pm 0.01 \mathrm{~mm}$ for the parallelepipedic TC. Just taking into account the uncertainty in the gap dimension $\left(L_{x}\right)$ leads to an relative systematic error in the order of $2 \%$ and $2.7 \%$ for the cylindrical and parallelepipedic TC.

The mass separation between the two ends of the column $\Delta c$ is determined from a calibration curve which relates mass fraction and density. In order to make the calibration, five mixtures with known concentration, close to the initial mass fraction $\left(c_{0} \pm 0.02\right)$ are prepared 

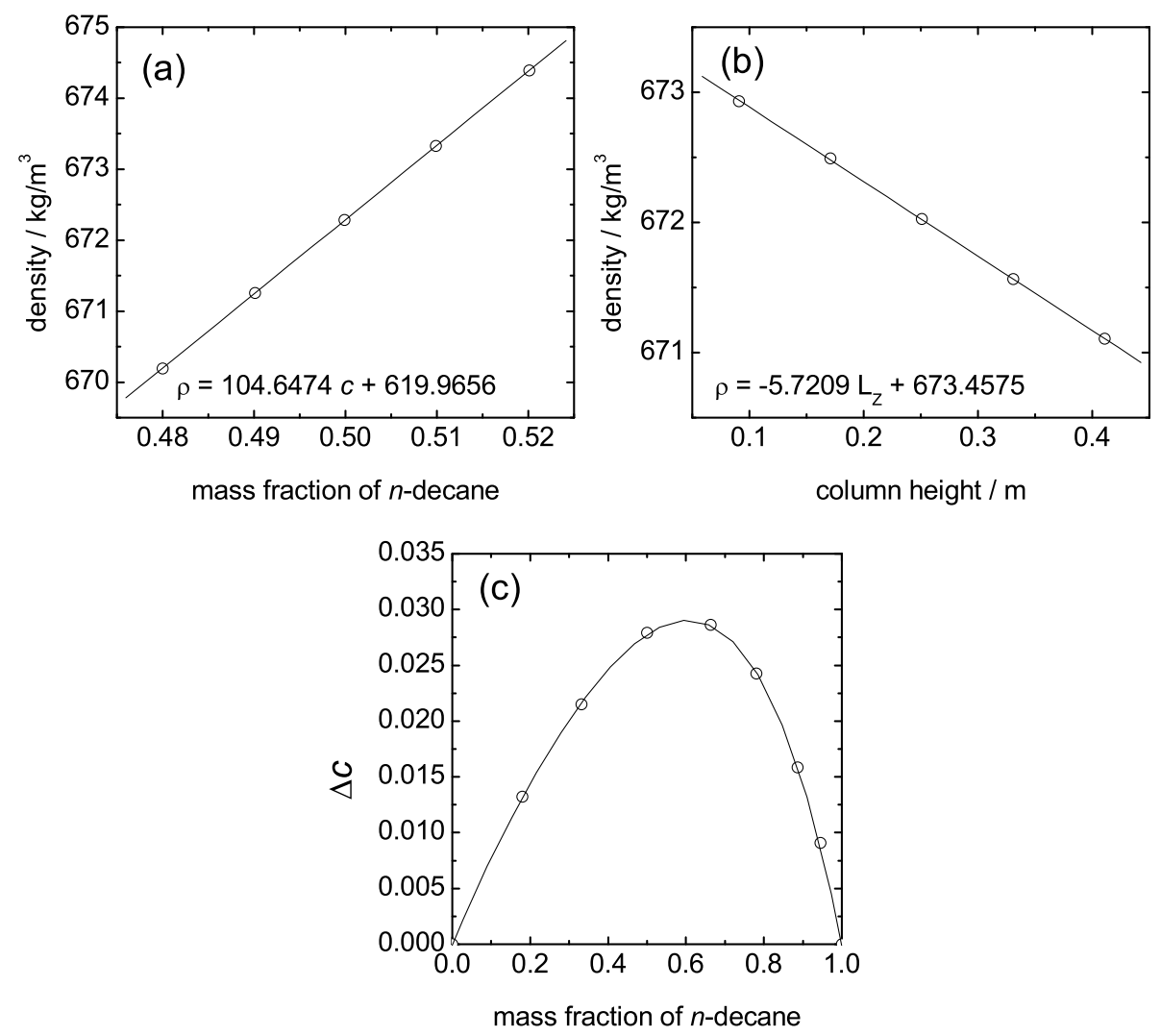

Figure 3.1: (a) Density of the mixture $n$-decane/n-pentane as function of the mass fraction around the concentration $c=0.5$ at $T=25^{\circ} \mathrm{C}$. (b) Density of the same mixture as function of the column height at $c=0.5$ and $T=25^{\circ} \mathrm{C}$. The results were obtained with the cylindrical TC. (c) Mass separation $\Delta c$ for $n$-decane/n-pentane as function of the mass fraction of $n$-decane at $T=27^{\circ} \mathrm{C}$. 
Table 3.1: Thermophysical properties of binary $n$-alkane mixtures with $n$-decane as first component. Chemical structure of the second component, mass fraction of $n$-decane $c$, density $\rho$, thermal expansion coefficient $\alpha$, mass expansion coefficient $\beta$, dynamic viscosity $\eta$, refractive index increment with concentration $(\partial n / \partial c)$ and temperature $(\partial n / \partial T)$.

\begin{tabular}{|c|c|c|c|c|c|c|c|}
\hline $\begin{array}{c}\text { second } \\
\text { component }\end{array}$ & $c$ & $\begin{array}{c}\rho / \\
\mathrm{kg} / \mathrm{m}^{3}\end{array}$ & $\begin{array}{c}\alpha / \\
10^{-3} \mathrm{~K}^{-1}\end{array}$ & $\beta$ & $\begin{array}{c}\eta / \\
\mathrm{m} \cdot \mathrm{Pa} \cdot \mathrm{s}\end{array}$ & $(\partial n / \partial c)$ & $\begin{array}{c}(\partial n / \partial T) / \\
10^{-3}\end{array}$ \\
\hline \multicolumn{8}{|c|}{$T=27^{\circ} \mathrm{C}$} \\
\hline \multirow{7}{*}{$\mathrm{C}_{5} \mathrm{H}_{12}$} & 0.945 & 718.461 & 1.069 & 0.1540 & 0.758 & - & - \\
\hline & 0.886 & 711.864 & 1.098 & 0.1571 & 0.690 & 0.05670 & -0.476 \\
\hline & 0.780 & 700.261 & 1.150 & 0.1568 & 0.586 & 0.05663 & -0.501 \\
\hline & 0.663 & 687.589 & 1.208 & 0.1555 & 0.478 & 0.05593 & -0.518 \\
\hline & 0.500 & 670.325 & 1.297 & 0.1571 & 0.403 & - & - \\
\hline & 0.332 & 652.835 & 1.399 & 0.1570 & 0.333 & 0.05388 & -0.519 \\
\hline & 0.180 & 637.718 & 1.495 & 0.1583 & 0.277 & - & - \\
\hline \multicolumn{8}{|c|}{$T=25^{\circ} \mathrm{C}$} \\
\hline $\mathrm{C}_{5} \mathrm{H}_{12}$ & & 672.306 & 1.288 & 0.1571 & 0.399 & 0.05481 & -0.504 \\
\hline $\mathrm{C}_{6} \mathrm{H}_{14}$ & & 689.823 & 1.200 & 0.1032 & 0.470 & 0.03724 & -0.494 \\
\hline $\mathrm{C}_{7} \mathrm{H}_{16}$ & & 702.601 & 1.141 & 0.0660 & 0.563 & 0.02425 & -0.477 \\
\hline $\mathrm{C}_{8} \mathrm{H}_{18}$ & & 712.330 & 1.098 & 0.0394 & 0.656 & 0.01435 & -0.466 \\
\hline $\mathrm{C}_{14} \mathrm{H}_{30}$ & 0.500 & - & - & - & - & -0.01723 & -0.434 \\
\hline $\mathrm{C}_{15} \mathrm{H}_{32}$ & & 745.446 & 0.974 & -0.0524 & 1.397 & - & - \\
\hline $\mathrm{C}_{16} \mathrm{H}_{34}$ & & 747.768 & 0.967 & -0.0588 & 1.515 & -0.02290 & -0.431 \\
\hline $\mathrm{C}_{17} \mathrm{H}_{36}$ & & 749.893 & 0.958 & -0.0645 & 1.634 & -0.02507 & -0.429 \\
\hline $\mathrm{C}_{18} \mathrm{H}_{38}$ & & 751.756 & 0.951 & -0.0698 & 1.778 & -0.02716 & -0.427 \\
\hline $\mathrm{C}_{20} \mathrm{H}_{42}$ & & 754.988 & 0.944 & -0.0781 & 2.102 & -0.03108 & -0.423 \\
\hline
\end{tabular}


by weighting. The accuracy of the determined mass is $0.0001 \mathrm{~g}$. For the investigated mixtures we obtained always a linear relation between the density and the mass fraction. From the calibration curve the mass expansion coefficient $\beta=(1 / \rho)(\partial \rho / \partial c)$ is obtained. An example for the mixture $n$-decane/ $n$-pentane is shown in the figure $3.1 \mathrm{a}$.

We determine the stationary mass separation between the two ends of the column using the following expression:

$$
\Delta c=\frac{L_{z}}{\beta \rho} \frac{\partial \rho}{\partial z}
$$

where $\partial \rho / \partial z$ is the vertical density gradient along the TC. The density gradient $\partial \rho / \partial z$ is obtained from five samples which are equally distributed along the height of the TC. In all studied mixtures the variation of the density with height is linear. A typical experimental result is shown in figure $3.1 \mathrm{~b}$.

The stationary state is determined by the following expression [20]:

$$
t_{r}=\frac{9 !\left(L_{z} v\right)^{2} D}{\left(g \pi \alpha \Delta T L_{x}^{3}\right)^{2}}
$$

where $t_{r}$ is the relaxation time and $\Delta T$ is the applied temperature difference between the two vertical walls. $\Delta T$ has been adjusted to $6^{\circ} \mathrm{C}$, although in the stationary state the mass separation is independent of the applied temperature gradient [168]. Typically the time for reaching the stationary state is 5 times the relaxation time. We have repeated each measurement at least 3 times and in one measurement we waited 15 times the relaxation time. All experimental results agreed with $2 \%$, which indicates that the chosen time, has been long enough to reach the stationary state.

Figure 3.1c shows the mass separation of the mixtures $n$-decane/ $n$-pentane at different initial mass fractions. The separation $\Delta c$ shows a maximum at a mass fraction of $n$-decane at $c=0.6$.

\section{TDFRS:}

The thermal diffusion behavior of the solutions was investigated by thermal diffusion forced Rayleigh scattering (TDFRS). A detailed description of the set-up can be found elsewhere [109]. 
The heterodyne diffraction signal $\zeta_{\text {het }}$ is evaluated by the equation,

$$
\zeta_{\text {het }}(t)=1+\left(\frac{\partial n}{\partial T}\right)^{-1}\left(\frac{\partial n}{\partial c}\right) S_{\mathrm{T}} c(1-c)\left(1-e^{-q^{2} D t}\right),
$$

with the refractive index increment with concentration at constant pressure and temperature $(\partial n / \partial c)$, the derivative of the refractive index with temperature at constant pressure and concentration $(\partial n / \partial T)$ and the collective diffusion coefficient $D$.

\subsubsection{Density measurements}

The thermal expansion $\alpha$, the mass expansion $\beta$ and the density $\rho$ of all the mixtures have been measured with an Anton Paar DMA 5000 vibrating quartz U-tube densimeter. It has a reproducibility of $1 \cdot 10^{-6} \mathrm{~g} / \mathrm{cm}^{3}$ with a temperature accuracy of $0.001^{\circ} \mathrm{C}$. The sample volume needed to make one density measurement is roughly $1.5 \mathrm{ml}$. The thermophysical properties of all studied mixtures are shown in Table 3.1.

\subsubsection{Viscosity measurements}

The dynamic viscosity has been determined by a HAAKE falling ball viscosimeter with an estimated accuracy of $\pm 1 \%$. The temperature stability is $\pm 0.1^{\circ} \mathrm{C}$. The volume needed to make one viscosity measurement is approximately $40 \mathrm{ml}$. The dynamic viscosity $\mu$ listed in Table 3.1 are the average of at least 8 individual measurements with typical standard deviation below $1 \%$.

\subsubsection{Refractive index increments}

An Anton Paar RXA 156 refractometer has been used to measure the refractive index increments with the mass concentration $(\partial n / \partial c)$ (see Table 3.1). It has a repeatability of $2 \cdot 10^{-5}$ and the temperature accuracy is $\pm 0.01^{\circ} \mathrm{C}$. The volume needed to make one measurement is less than $1 \mathrm{ml}$. For all investigated temperatures and concentrations we find a linear dependence of the refractive index on concentration if the temperature is fixed or on temperature if the concentration is fixed. For all mixtures we determined the $(\partial n / \partial c)$ values. We would like to point out that the refractive index increments with concentration, which had been 
Table 3.2: Thermal diffusion coefficients $\left(10^{-8} \mathrm{~cm}^{2} \mathrm{~s}^{-1} \mathrm{~K}^{-1}\right)$ for $n$-decane in $n$-pentane at $T=27^{\circ} \mathrm{C} . D_{\mathrm{T}}^{\mathrm{TDFRS}}{ }_{\text {old }}$ and $D_{\mathrm{T}}^{\mathrm{S}-\mathrm{NEMD}_{\text {c.v. }}}$ refer to experimental data and simulation results in the center-of-volume-reference frame, respectively [113]. $D_{\mathrm{T}}^{\mathrm{TC}}{ }^{\mathrm{TC} a r a}, D_{\mathrm{T}}^{\mathrm{TC}}{ }^{\mathrm{T} y l}$ and $D_{\mathrm{T}}^{\mathrm{TDFRS}}$ have been measured by parallelepipedic TC, cylindrical TC and TDFRS. For details see the text.

\begin{tabular}{|c|c|c|c|c|c|c|}
\hline$x$ & $c$ & $\begin{array}{c}D_{\mathrm{T}}^{\mathrm{TDFRS}} \text { old } \\
(\text { Ref. [113] })\end{array}$ & $\begin{array}{c}D_{\mathrm{T}}^{\mathrm{S}-\mathrm{NEMD}_{c . v .}} \\
(\text { Ref. [113] })\end{array}$ & $D_{\mathrm{T}}^{\mathrm{TC} \text { para }}$ & $D_{\mathrm{T}}^{\mathrm{TC} \text { cyl }}$ & $D_{\mathrm{T}}^{\mathrm{TDFRS}}$ \\
\hline 0.10 & 0.180 & - & - & - & $11.99 \pm 0.5$ & - \\
0.20 & 0.332 & $9.28 \pm 0.75$ & $13.30 \pm 0.97$ & $10.49 \pm 0.03$ & $10.36 \pm 0.5$ & $10.81 \pm 0.7$ \\
0.34 & 0.180 & - & - & - & $9.37 \pm 0.4$ & - \\
0.50 & 0.663 & $7.54 \pm 0.61$ & $10.42 \pm 2.34$ & $8.76 \pm 0.03$ & $8.67 \pm 0.4$ & $9.11 \pm 0.6$ \\
0.64 & 0.780 & - & - & - & $7.56 \pm 0.3$ & $8.11 \pm 0.7$ \\
0.80 & 0.886 & $7.18 \pm 0.59$ & $10.16 \pm 1.50$ & $6.76 \pm 0.02$ & $6.92 \pm 0.3$ & $7.75 \pm 0.6$ \\
0.90 & 0.945 & - & - & - & $7.02 \pm 0.4$ & - \\
\hline
\end{tabular}

determined for the mixture $n$-decane/ $n$-pentane in the previous work [113] by an Abbe refractometer agreed with the new values within the error bars.

For the TDFRS measurements for all mixtures except for the system $n$-decane/ $n$-pentane $(\partial n / \partial T)$ was directly measured by an interferometer. In the case of $n$-decane/n-pentane $(\partial n / \partial T)$ the reliability of the refractometer was better, because due to the long measurement time in the interferometer pentane evaporated partly, which lead to concentration changes during the measurement. The contrast factors $(\partial n / \partial c)$ and $(\partial n / \partial T)$ for two groups of mixtures are listed in Table 3.1 .

\subsection{Results and discussion}

\subsubsection{Thermal diffusion behavior of $n$-decane in $n$-pentane}

Figure 3.2 shows the thermal diffusion coefficient $D_{\mathrm{T}}$ for $n$-decane in $n$-pentane for several mass fractions of $n$-decane. In general $D_{\mathrm{T}}$ decays with increasing $n$-decane content. The measurements between the parallelepipedic and cylindrical thermogravitational columns agree 


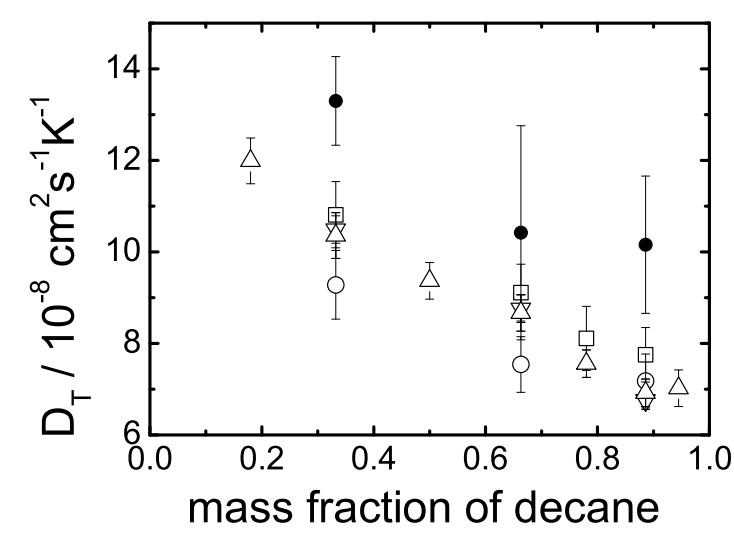

Figure 3.2: Thermal diffusion coefficient $D_{\mathrm{T}}$ for $n$-decane in $n$-pentane in dependence of the mass fraction of $n$-decane obtained by a parallelepipedic TC $(\nabla)$, cylindrical TC $(\triangle)$ and TDFRS $(\square)$. For comparison we show also the previous experimental $(\bigcirc)$ and simulation results in the center-of-volume-reference frame $(\bullet)$ [113].

typically better than $5 \%$. We estimated the error bars for the TC by error propagation taking into account the experimental uncertainties of the auxiluary quantities such as viscosity $(<1 \%)$, mass expansion $(<1 \%)$, thermal expansion $(<0.5 \%)$, variation of the density with height in the column (typically better than $2 \%$ and $3 \%$ for cylindrical and parallelepipedic TC, respectively) and geometrical parameters (typically better than $1 \%$ and $3 \%$ for cylindrical and parallelepipedic TC). The error bars for the TDFRS data correspond to one standard deviation of the mean for repeated measurements. The actual TDFRS data are systematically 5-11\% higher than the TC data, but agree within the error bars. The highest deviation in comparison with the TDFRS data of $11 \%$ has been found for the lowest pentane content. This concentration is the one, which is most sensitive to the evaporation of pentane. The same absolute loss of pentane leads for this concentration to a much larger relative concentration change compared to concentrations with a higher pentane content. In both experiments a potential loss of pentane was carefully monitored as described in Section 3.2.1. The expected changes in concentration are smaller than the symbol size. In addition, the thermal diffusion 
coefficient of $n$-decane in $n$-pentane mixtures for different concentrations deviate less than $3 \%$ from the values obtained with another cylindrical column [3], which is an independent measurement by another group.

The old TDFRS measurements [113] are systematically 10-20\% lower than the present TDFRS data and the deviation with the TC data are in the order of 5-15\%. The deviations between the two sets of TDFRS data can probably be explained by the fact that at that time the data had not been corrected by the so-called excitation function which accounts for time delays in the electrical switching of the Pockels cell. A detailed description of the procedure can be found in Ref. [109].

We compare also our new TC and TDFRS data with previous non-equilbrium molecular dynamic simulation results for the system $n$-decane/ $n$-pentane by Perronace et al. [113] (see Table 3.2). The simulations have been carried out in the center-of-mass reference frame and the resulting transport coefficients have been transformed to the center-of-volume reference frame, which corresponds to the situation in the experiment. The statistical uncertainty of the simulations is in the order of $35 \%$, while the systematic deviations between experimental and simulation data are in the order of $15-40 \%$. For instance for the equimolar mixture $n$ decane/ $n$-pentane the data agree almost within the error bars.

\subsubsection{Thermal diffusion behavior of $n$-decane in various alkane at equal mass ratio}

Additionally we performed measurements for $n$-decane with various shorter and longer linear alkanes. The thermal diffusion and diffusion coefficients for $n$-decane in various alkanes with a mass fraction of $50 \%$ at $T=25^{\circ} \mathrm{C}$ are listed in Table 3.3. For comparison we list also previous thermal diffusion data, which have also been obtained by a parallelepipedic TC but with different dimensions [85]. The diffusion coefficients from the same reference [85] have been determined by the open-end capillary (OEC) method [8].

Figure 3.3(a) shows the thermal diffusion, diffusion and Soret coefficients for the measurement with the cylindrical TC and the TDFRS set-up as function of the molar mass of the second component. For comparison we also show the previous data by Leahy-Dios and Firoozabadi [85]. As expected the thermal diffusion coefficient of $n$-decane in shorter alkane is positive, and therefore the $n$-decane goes towards the cold region, while it becomes neg- 
Table 3.3: Thermal diffusion and diffusion coefficients for $n$-decane in various alkanes at $T=25^{\circ} \mathrm{C}$. In the first two columns the totals formula and the molecular weight of the second component are listed. $D_{\mathrm{T}}^{\mathrm{TC}}$ and $D^{\mathrm{OEC}}$ refer to previous experimental data [85]. $D_{\mathrm{T}}^{\mathrm{TC}_{\mathrm{cyl}}}$ and $D_{\mathrm{T}}^{\mathrm{TDFRS}}$ have been measured by cylindrical TC and TDFRS. Details are given in the text.

\begin{tabular}{|c|c|c|c|c|c|}
\hline totals & $\begin{array}{c}D_{\mathrm{T}}^{\mathrm{TC}} / 10^{-8} \\
\mathrm{~cm}^{2} \mathrm{~s}^{-1} \mathrm{~K}^{-1}\end{array}$ & $\begin{array}{c}D^{\mathrm{OEC}} / 10^{-5} \\
\mathrm{~cm}^{2} \mathrm{~s}^{-1}\end{array}$ & $\begin{array}{c}D_{\mathrm{T}}^{\mathrm{TC}} \\
\mathrm{cm}^{2} \mathrm{~s}^{-1} \mathrm{~K}^{-1}\end{array}$ & $\begin{array}{c}D_{\mathrm{T}}^{\mathrm{TDFRS}} / 10^{-8} \\
\mathrm{~cm}^{2} \mathrm{~s}^{-1} \mathrm{~K}^{-1}\end{array}$ & $D^{\mathrm{TDFRS}} / 10^{-5}$ \\
$\mathrm{~cm}^{2} \mathrm{~s}^{-1}$ \\
\hline $\mathrm{C}_{5} \mathrm{H}_{12}$ & $9.64 \pm 0.19$ & $2.50 \pm 0.20$ & $9.24 \pm 0.4$ & $9.59 \pm 0.33$ & $3.19 \pm 0.04$ \\
$\mathrm{C}_{6} \mathrm{H}_{14}$ & $7.79 \pm 0.21$ & - & $6.71 \pm 0.3$ & $6.51 \pm 0.29$ & $2.69 \pm 0.07$ \\
$\mathrm{C}_{7} \mathrm{H}_{16}$ & $5.99 \pm 0.56$ & $2.23 \pm 0.11$ & $4.37 \pm 0.3$ & $5.00 \pm 0.42$ & $2.61 \pm 0.20$ \\
$\mathrm{C}_{8} \mathrm{H}_{18}$ & $3.86 \pm 0.14$ & $1.84 \pm 0.18$ & - & $3.51 \pm 0.53$ & $2.57 \pm 0.27$ \\
$\mathrm{C}_{12} \mathrm{H}_{26}$ & $-1.85 \pm 0.41$ & $1.18 \pm 0.12$ & - & - & - \\
$\mathrm{C}_{14} \mathrm{H}_{30}$ & $-2.65 \pm 0.22$ & $0.90 \pm 0.22$ & - & $-1.85 \pm 0.46$ & $0.89 \pm 0.24$ \\
$\mathrm{C}_{15} \mathrm{H}_{32}$ & - & - & $-2.39 \pm 0.15$ & - & - \\
$\mathrm{C}_{16} \mathrm{H}_{34}$ & $-3.35 \pm 0.09$ & $0.68 \pm 0.07$ & $-2.47 \pm 0.15$ & $-2.58 \pm 0.29$ & $0.84 \pm 0.12$ \\
$\mathrm{C}_{17} \mathrm{H}_{36}$ & - & - & $-2.53 \pm 0.15$ & $-2.59 \pm 0.17$ & $0.75 \pm 0.07$ \\
$\mathrm{C}_{18} \mathrm{H}_{38}$ & - & - & $-2.56 \pm 0.15$ & $-2.69 \pm 0.17$ & $0.73 \pm 0.05$ \\
$\mathrm{C}_{20} \mathrm{H}_{42}$ & $-2.31 \pm 0.04$ & $0.47 \pm 0.01$ & $-2.65 \pm 0.15$ & $-2.86 \pm 0.12$ & $0.65 \pm 0.03$ \\
\hline
\end{tabular}




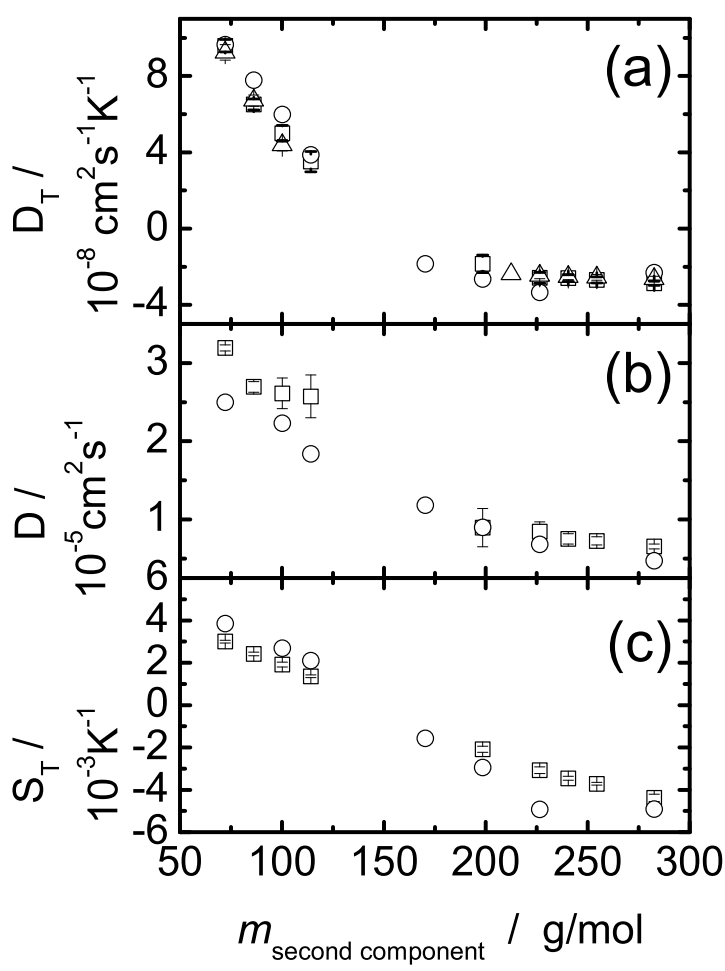

Figure 3.3: Thermal diffusion coefficient $D_{\mathrm{T}}$, diffusion coefficient $D$ and Soret coefficient $S_{\mathrm{T}}$ for $n$-decane in different alkanes in dependence of the molar mass of the second alkane component measured by TC $(\triangle)$ and TDFRS $(\square)$. For comparison we show also the data $(\bigcirc)$ obtained in the previous work by Leahy-Dios and Firoozabadi [85]. All measurements have been performed for $c=0.5$ at a temperature $T=25^{\circ} \mathrm{C}$.

ative when the mass of the second component becomes larger, which implies that $n$-decane migrates to the warm side. The agreement between the cylindrical TC and the TDFRS data is typically better than $5 \%$.

If we compare our data with recent data in the literature [85], we find deviations between $10-30 \%$. Compared to those previous measurements the TC used in this work allows a more 
accurate analysis of the mass separation between the top and the bottom of the TC due to the smaller gap and a better precision of the gap of $L_{x}=1.0 \pm 0.005 \mathrm{~mm}$ (c.f. 3.2.2). The TC used by Leahy-Dios and Firoozabadi [85] had a gap of $1.6 \pm 0.02 \mathrm{~mm}$. This low precision of the gap dimensions causes an uncertainty of $5 \%$ in the determination of the thermal diffusion coefficient, not regarding the propagating errors due to uncertainties in the thermophysical properties, which are required to calculate the thermal diffusion coefficient (see Eqs. 3.2 and 3.3). For the TC used in this work the mass separation is 6.55 times greater than for the TC used in Ref. [85]. Therefore, the difference in the thermal diffusion coefficient, $D_{\mathrm{T}}$, determined with those two columns (Ref. [85] and this work) becomes larger for mixtures with a small mass separation. This tendency can be observed for the mixtures $n$-decane/ $n$-heptane and $n$-decane $/ n$-hexadecane. In contrast to the previous measurements we could not confirm the non-monotonic trend of the thermal diffusion coefficient with increasing molecular weight of the second component. Both measurement techniques, TC and TDFRS indicate that $D_{\mathrm{T}}$ becomes constant and therefore independent of the molecular weight of the second component. This is also the behavior, which has been observed for infinite diluted solutions of polymers [145, 124].

Figure 3.3(b) shows a comparison between the diffusion coefficients determined in the previous study by the open-end-capillary (OEC) technique and the present TDFRS study. In general the OEC data are systematically lower than for the TDFRS data. Typically one finds deviations larger than $30 \%$ in the entire molar mass range of the second compound. Typically the agreement is better for the higher molar mass components than the lower molecular weight components, therefore evaporation problems might be responsible for these discrepancies.

In Figure 3.3c we compare the Soret coefficients determined by the TDFRS method with the previous results by Leahy-Dios and Firoozabadi [85]. Both studies show a decay of the Soret coefficient with increasing molecular weight of the second component. While the previous data seem to show a vague minimum, our data do not confirm this tren. The magnitude of Soret coefficient calculated [85] from the thermal diffusion coefficient determined by the TC method and diffusion coefficients measured with the OEC method is always larger than the $S_{\mathrm{T}}$ values determined in the TDFRS experiment. The deviations are typically in the order of $20-40 \%$. 


\subsection{Conclusion}

In this chapter, we studied systematically binary alkane mixtures by two different techniques, a convective method the thermogravitational column (TC) (paralelepipedic and cylindrical configurations) technique and a non-convective method thermal diffusion forced Rayleigh scattering (TDFRS). In general we found a very good agreement between these two methods. Nevertheless we found some discrepancies with data published in the literature. The observed disagreement between the published TDFRS [113] data for the mixture $n$-decane/ $n$-pentane is probably caused by an improved data analysis algorithm, which accounts for finite rising times and slow drifts of the electro-optic devices used in the experiment. Additionally we found discrepancies with recently published TC data [85]. We assume that the reason for the disagreement of the recent TC data is the larger gap $L_{x}$ in the previously used cell [85] compared to the cells used in this work. The larger gap decreases the accuracy of determining the mass separation between the two ends of the TC. This is especially important for the mixtures of decane, with the higher alkanes. Neither our TC nor our TDFRS measurements showed the upward trend of the thermal diffusion coefficient, $D_{\mathrm{T}}$, for the higher alkanes, which was recently observed [85]. Our measurements seem to indicate that the thermal diffusion coefficient becomes independent of the molar mass of the second component. This issue could certainly be further investigated by molecular simulations as it has been done for alkane mixtures [113] and other small molecules (c.f. Chapter 2). 


\section{Mixtures of alkane in benzene: experiment and lattice calculations}

In this chapter the thermal diffusion behavior of binary mixtures of linear alkanes in benzene has been investigated by thermal diffusion forced Rayleigh scattering (TDFRS) for a range of concentrations and temperatures. The Soret coefficient $S_{T}$ of the alkane was found to be negative for these $n$-alkane/benzene mixtures indicating that the alkanes are enriched in the warmer regions of the liquid mixtures. For the investigated compositions, the magnitude of the Soret coefficient decreases with increasing chain length and increasing alkane content of the mixtures. The temperature dependence of the Soret coefficient depends on mixture composition and alkane chain length; the slope of $S_{T}$ versus temperature changes from positive to negative with increasing chain length at intermediate compositions. To study the influence of molecular architecture on the Soret effect, mixtures of branched alkanes in benzene were also investigated. Our results for the Soret coefficients show that the tendency for the alkanes to move to the warmer regions of the fluid decreases with increasing degree of branching. The branching effect is so strong that for 2,2,4-trimethylpentane/benzene mixtures the Soret coefficient changes sign at high alkane content and that equimolar 2,2,3-trimethylbutane/benzene mixtures have positive Soret coefficients in the investigated temperature range. In order to investigate the effect of molecular interactions on thermal diffusion, we adapted a recently developed two-chamber lattice model to $n$-alkane/benzene mixtures. The model includes the effects of chain-length, compressibility, and orientation dependence of benzene-benzene 
interactions and yields good qualitative predictions for the Soret effect in $n$ alkane/benzene mixtures. For the branched isomers, we find some correlations between the moments of inertia of the molecules and the Soret coefficients.

\subsection{Introduction}

Thermal diffusion or the so-called Ludwig-Soret effect describes the coupling between a temperature gradient and a resulting mass flux. The effect has important technical applications for example in the modeling of the separation of crude oil components under the influence of thermal diffusion in geological conditions $[30,57,58]$; it also plays an important role in separation techniques for liquid mixtures (see e.g. Refs. [76, 77, 179]).

According to the phenomenological equations of irreversible thermodynamics, thermodiffusion in a binary fluid mixture is described by the flux of one of the components in response to a temperature and concentration gradient [35]. For an alkane/benzene mixture, for example, the flux $\mathbf{J}$ of the alkane in response to a temperature gradient $\nabla T$ and a mass-fraction gradient $\nabla w$ may be written as [35],

$$
\mathbf{J}=-\rho D \nabla w-\rho D_{T} w(1-w) \nabla T
$$

where $w$ is the mass fraction of the alkane, $\rho$ is the density of the mixture, $D$ is the mutual diffusion coefficient, and $D_{\mathrm{T}}$ is the thermal diffusion coefficient of the alkane. In the steady state $(J=0)$ the concentration gradient is characterized by the Soret coefficient $S_{\mathrm{T}}=D_{\mathrm{T}} / D$ of the alkane; a positive Soret coefficient of the alkane corresponds to the alkane moving to the colder regions of the fluid [52, 181].

Thermal diffusion in liquid mixtures of non-polar fluids is known to reflect a range of microscopic properties such as the mass, size, and shape of the molecules as well as their interactions (see Ref. [179] for a review). In mixtures of polar liquids, specific interactions between the molecules dominate the thermal diffusion process while mass and size of the molecules are most important in Lennard-Jones fluids. For liquids of non-polar molecules that are more complex than Lennard-Jones fluids, the Soret effect appears to depend on a delicate balance of the molecular properties of the components.

* The work described in this chapter is based on J. Phys. Chem. B 110, 26215-26224 (2006) 
This is true, in particular, for mixtures of alkanes and aromatic solvents. Debuschewitz and Köhler [36] investigated thermal diffusion in isotopic mixtures of benzene and cyclohexane and found that the Soret coefficient could be written as a sum of three contributions:

$$
S_{T}=a_{M} \delta M+b_{I} \delta I+S_{T}^{0}
$$

where $\delta M=\left(M_{1}-M_{2}\right)\left(M_{1}+M_{2}\right)^{-1}$ and $\delta I=\left(I_{1}-I_{2}\right)\left(I_{1}+I_{2}\right)^{-1}$ are the relative differences of the masses $\left(M_{1}, M_{2}\right)$ and moments of inertia $\left(I_{1}, I_{2}\right)$ of the molecules, respectively. The coefficients $a_{M}$ and $b_{I}$ were found to be independent of the composition of the mixture. The third contribution, $S_{\mathrm{T}}^{0}$, reflects chemical differences of the molecules and was found to depend on the concentration and change its sign at a benzene mole fraction of 0.7. A further investigation of the isotope effect [183] suggested that the absolute rather than the relative differences between the masses and moments of inertia should enter the expression for the Soret coefficient so that the difference terms in Eq. (4.2) are given by $\delta M=M_{1}-M_{2}$ and $\delta I=I_{1}-I_{2}$.

A number of studies have focused on the Soret effect in mixtures containing an alkane or benzene as one of the components. Different experimental techniques were applied to investigate the thermal diffusion behavior of toluene/hexane [188, 46, 75], alkane/alkane (c.f. Chapter 3), cyclohexane/benzene [36], and $n$-alkane/benzene [81, 80, 37, 46, 165, 20] mixtures. The three binary mixtures of dodecane, isobutylbenzene and 1,2,3,4 tetrahydronaphthalene [116] were the subject of a benchmark study to provide reliable values for the Soret, diffusion and thermal diffusion coefficients of these mixtures. Rowley et al. [132, 131] measured the heat of transport of binary mixtures of six alkanes ( $n$-hexane, $n$-heptane, $n$-octane, 3-methylpentane, 2,3-dimethylpentane, 2,2,4-trimethylpentane) with chloroform and tetrachloride in a wide concentration range at $30^{\circ} \mathrm{C}$. Demirel and Sandler [38] combined these data to determine values of thermal diffusion ratios, $K_{T}=w(1-w) S_{\mathrm{T}}$, for these mixtures. They found that branching had a very small effect on the thermal diffusion ratios. For mixtures of pentane and decane, Perronace et al. [113] determined Soret coefficients from both experiment and molecular dynamics simulations and found reasonable agreement between experiment and simulations.

Thermal diffusion in hydrocarbon mixtures has also been investigated with theoretical models based on thermodynamic considerations. Gonzalez-Bagnoli et al. [61] compared results for the thermal diffusion factor, $\alpha=T S_{\mathrm{T}}$, obtained from seven thermodynamic models 
for the Soret effect with experimental data for three mixtures. They found that three of the older models did not provide good results for any of the mixtures and that the remaining four models had varying success in predicting the sign and magnitude of the thermal diffusion factors. Part of the problem is the quality of the equation of state. A comparison of results from different equations of state showed, in agreement with earlier work [70], that the partial molar quantities have a large effect on the calculated values of the thermodiffusion factors. However, it was also found that an improvement in the representation of the partial molar quantities does not typically lead to improved predictions for the Soret effect [61].

We focus in the present chapter on the Soret effect in binary alkane/benzene mixtures for linear as well as branched alkanes. Since benzene is a very good solvent for alkanes, we are able to explore the composition as well as temperature dependence of the Soret effect. For the $n$-alkanes, we expect the differences in the chain length (molecular mass) to have the largest effect; for the branched alkanes, we expect a significant effect due to differences in the molecular architecture and the corresponding changes in the moments of inertia. However chemical differences will also be important since the type of covalently bonded neighbors affects the interactions between carbon atoms (see e.g. Refs. [133, 27]). In addition, ordering effects in the liquid may play a role. Interactions between benzene molecules are known to depend on the relative orientation of the molecules (see e.g. Refs. [148, 191, 176, 23]). In the benzene solid at atmospheric pressure, the relative orientation of benzene molecules corresponds to the "T" configuration [148, 191], where the rings of the molecules are perpendicular to each other and the center of the second molecule lies on the normal through the center of the ring of the first molecule $[176,23]$. Since the melting temperature of benzene $(278.7 \mathrm{~K})$ is close to the temperature of the experiments, the orientation dependence of the interactions is expected to affect the thermophysical properties of alkane/benzene mixtures [135]. The chains of normal alkanes also show some orientational order in the liquid state near the melting temperature $[53,21]$. While this effect is expected to be negligible for most of the alkanes investigated in this chapter (their melting temperature is well below room temperature) it may affect thermodiffusion for the long-chain alkanes. Since interactions between molecules play such an important role in thermal diffusion a systematic investigation of the alkane/benzene mixtures will aid the development of molecular models for computer simulations, which have recently become an important tool in the investigation of the Soret effect (see, for example, 
$2-\mathrm{MH}$<smiles>CCCCC(C)C</smiles>

2,3-DMP<smiles>CCC(C)C(C)C</smiles>

2,2,3-TMB

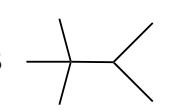

3-MH<smiles>CCCC(C)CC</smiles>

2,4-DMP<smiles>CC(C)CC(C)C</smiles>

2,2,4-TMP

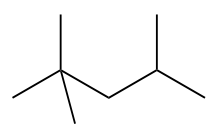

Figure 4.1: Chemical structure of the investigated isomers: 2-methylhexane (2-MH), 3methylhexane (3-MH), 2,3-dimethylpentane (2,3-DMP), 2,4-dimethylpentane (2,4-DMP), 2,2,3-trimethylbutane (2,2,3-TMB), 2,2,4-trimethylpentane (2,2,4-TMP).

Refs. $[113,130,189,104)$.

In this chapter, the thermal diffusion behavior of alkane/benzene mixtures is investigated experimentally with thermal diffusion forced Raleigh scattering (TDFRS). Experiments were performed on mixtures of benzene with the linear alkanes heptane, nonane, undecane, tridecane, pentadecane, and heptadecane and with five isomers of heptane, namely 2methylhexane, 3-methylhexane, 2,3-dimethylpentane, 2,4-dimethylpentane, and 2,2,3-trimethylbutane, and one isomer of octane, 2,2,4-trimethylpentane. The chemical structures of the investigated isomers are presented in Fig. 4.1. Experiments were performed in a temperature range from $20{ }^{\circ} \mathrm{C}$ to $40{ }^{\circ} \mathrm{C}$ and for blends with alkane mole fractions of $x=0.25,0.5$, 0.75 , and 0.85 and for a mass fraction of $x=0.05$. The experiments yield values for the Soret coefficients, $S_{\mathrm{T}}$, the mass diffusion coefficients, $D$, and the thermal diffusion coefficients $D_{\mathrm{T}}=S_{\mathrm{T}} D$. In order to investigate with theoretical methods the effect of intermolecular interactions on thermal diffusion, we have adapted a recently developed two-chamber lattice model for thermodiffusion $[90,91]$ to alkane/benzene mixtures. In the two-chamber lattice model, one considers a lattice system divided into two chambers of equal size that are maintained at slightly different temperatures. Particles are free to move between the chambers, which do not otherwise interact. The partition functions for the chambers are calculated in 
exact enumeration and combined to yield a sum of states for the system. The Soret coefficient is then determined from the difference in average composition of the solutions in the two chambers. The system-dependent parameters of the model are determined from a comparison with thermodynamic properties of the pure components and volume changes on mixing. This allows us to make predictions of the Soret coefficient as a function of temperature, pressure, and composition without adjustable parameters. We find that predictions from this model, which includes the effects of chain length of the alkanes and orientation-dependent interactions of benzene molecules, are in good qualitative agreement with the Soret coefficients of linear alkane chains in benzene.

The chapter is organized as follows: in the next section, Sec. 4.2, we describe briefly the sample preparation, as well as the TDFRS experiment and the index of refraction measurements necessary for the evaluation of the TDFRS signal. In Sec. 4.3 we present our experimental results for mixtures of benzene with linear and branched alkanes. In Sec. 4.4 we describe the two-chamber lattice model that is used to predict values of the Soret coefficients of $n$-alkane/benzene mixtures and we present a comparison with experimental data. Appendix 4.6 contains details about the determination of system-dependent parameters for the model. We discuss our results and conclusions in Sec. 4.5.

\subsection{Experiment}

\subsubsection{Sample preparation}

The alkanes heptane (99,5\%), nonane (99\%), undecane (98\%), 2-methylhexane (98\%), 3methylhexane (98\%) and 2,2,3-trimethylbutane (99\%) were purchased from Fluka; tridecane (99\%), pentadecane (99\%), heptadecane (99\%), 2,3-dimethylpentane (99\%), 2,4-dimethylpentane (99\%), 2,2,4-trimethylpentane (99\%) and benzene (99.7\%) were ordered from Aldrich. Fig. 4.1 shows the chemical structure of the investigated isomers. The alkane mole fraction for all mixtures was adjusted by weighing the components. The TDFRS experiments require a small amount of dye in the sample. All samples contained approximately $0.002 \mathrm{wt} \%$ of the dye Quinizarin (Aldrich). This amount ensures a sufficient optical modulation of the grating but is small enough to avoid convection and contributions of the dye to the concentration signal. Before each TDFRS experiment, approximately $2 \mathrm{ml}$ of the freshly prepared solu- 
tion were filtered through $0.2 \mu \mathrm{m}$ filter (hydrophobic PTFE) into an optical quartz cell with $0.2 \mathrm{~mm}$ optical path length (Helma) which was carefully cleaned from dust particles before usage.

\subsubsection{Refractive index increment measurements}

In order to determine the changes of the refractive index $n$ with blend composition $w$ at constant pressure $P$ and temperature $T,(\partial n / \partial w)_{P, T}$, for each alkane/benzene system measurements were performed with an Abbe refractometer for several mixture compositions around the desired molar fraction. The slope $(\partial n / \partial w)_{P, T}$ was then determined by linear interpolation. For instance, we measured the refractive index for seven concentration in the range between 0.35 to 0.75 in order to determine the slope $(\partial n / \partial w)_{P, T}$ for $x=0.5$. An analog procedure was used for intermediate molar fractions of $x=0.25$ and $x=0.75$. For measurements at very low alkane concentrations, it is important to perform the index of refraction measurements using benzene from the same lot as is used in the TDFRS experiments. The reason is that the index of refraction of benzene is very sensitive to impurities. Even for high grade benzene we observed a range of refractive index values for pure benzene (between 1.500 - 1.502 at room temperature) which may lead to significant changes in the measured refractive index increment for mixtures with low alkane content. For all mixture compositions investigated in this chapter, the temperature derivatives at constant pressure and composition, $(\partial n / \partial T)_{P, w}$, were determined from measurements with a Michelson interferometer [14] in a temperature range of $3{ }^{\circ} \mathrm{C}$ above and below the temperature of the TDFRS experiment.

\subsubsection{TDFRS experiment and data analysis}

In our thermal diffusion forced Raleigh scattering (TDFRS) experiments, the beam of an an argon-ion laser $\left(\lambda_{w}=488 \mathrm{~nm}\right)$ is split into two writing beams of equal intensity which are allowed to interfere in the sample cell (see Ref. [113] for a detailed description of the method). A small amount of dye is present in the sample and converts the intensity grating into a temperature grating, which in turn causes a concentration grating by the effect of thermal diffusion. Both gratings contribute to a combined refractive index grating, which is read out by Bragg diffraction of a third laser beam $\left(\lambda_{r}=633 \mathrm{~nm}\right)$. 


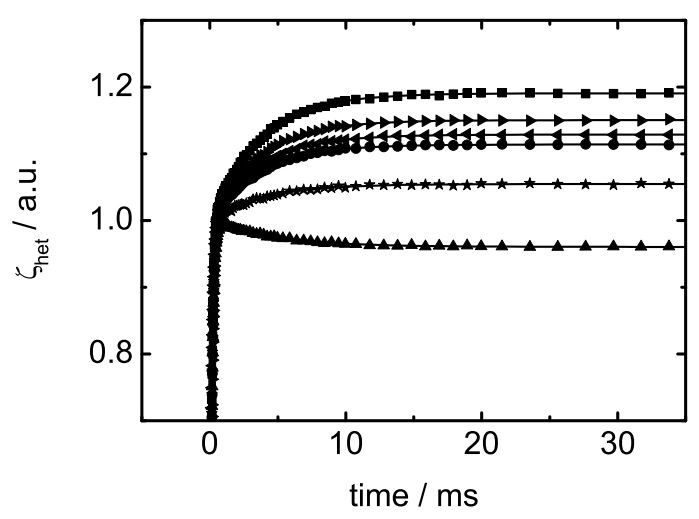

Figure 4.2: Typical normalized TDFRS signals for binary equimolar mixtures of heptane( $\mathbf{\square})$,

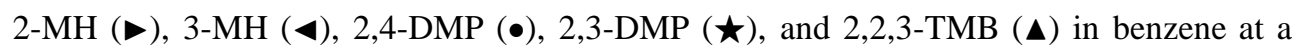
temperature of $30^{\circ} \mathrm{C}$. The solid lines represent the fits to Eq. (4.3).

Fig. 4.2 shows typical heterodyne signals of the read-out laser normalized to the thermal signal. The intensity $\zeta_{\text {het }}(t)$ of the signal depends on the transport coefficients and the index of refraction increments and may be expressed as

$$
\zeta_{\text {het }}(t)=1+\frac{(\partial n / \partial w)_{P, T}}{(\partial n / \partial T)_{P, w}} S_{\mathrm{T}} w(1-w)\left(1-e^{-q^{2} D t}\right) .
$$

where $q=4 \pi n / \lambda_{w} \sin (\theta / 2)$ is the grating vector, whose absolute value is determined by the angle $\theta$ between the two writing beams, the wavelength $\lambda_{w}$, and the index of refraction $n$.

For the determination of the transport coefficients, Eq. (4.3) is fitted to the measured heterodyne signal (see Fig. 4.2) using the independently measured contrast factors $(\partial n / \partial w)_{p, T}$ and $(\partial n / \partial T)_{p, w}$.

\subsection{Results}

In Fig. 4.3 we present results for the Soret coefficients $S_{\mathrm{T}}$, the mutual diffusion coefficients $D$, and the thermal diffusion coefficients, $D_{T}$, as a function of temperature for equimolar 
mixtures of benzene and the six $n$-alkanes considered in this chapter. For the heptadecane/benzene mixture, only the Soret coefficients are shown in Fig. 4.3 since the small amplitude of the signal at the lowest temperatures prevented a reliable determination of the mutual and thermal diffusion coefficients for this mixture. The Soret coefficients of the alkanes in Fig. 4.3 are negative which implies that the alkane molecules tend to move to the warmer regions of the fluid while the benzene molecules tend to move in the opposite direction. With increasing chain length, the Soret coefficients increase (their magnitude decreases) and the slope of the temperature dependence decreases becoming negative at the highest molecular weights. The diffusion coefficients $D$ increase with increasing temperature and decrease with increasing molecular weight of the alkane. The chain length dependence of the diffusion coefficients is expected since the size of the alkane molecules and the viscosity of the mixture increase with increasing molecular mass of the alkanes. Fig. 4.4 shows the transport coefficients $S_{\mathrm{T}}, D$, and $D_{T}$ as a function of alkane mole fraction for mixtures of benzene with heptane, tridecane, and the branched octane isomer 2,2,4-trimethylpentane (2,2,4-TMP). Experimental values for three different temperatures are shown for the Soret coefficients while experimental data for a single temperature are shown for the mutual and the thermal diffusion coefficients. For each of the mixture systems, the Soret coefficient increases with increasing alkane concentration. For heptane and tridecane, the Soret coefficients remain negative for all concentrations investigated here. For 2,2,4-TMP, however, the Soret coefficient changes sign near the alkane mole fraction of $x=0.75$ and is positive for $x=0.85$. The mutual diffusion coefficient $D$ increases with increasing alkane concentration. Soret coefficients of heptane/benzene mixtures have been measured independently by Korsching [81, 80], Demichowicz-Pigoniowa et al. [37], Ecenarro et al. [46] and Trevoy et al. [165] The results for a temperature of $25{ }^{\circ} \mathrm{C}$ are presented in Fig. 4.5 as a function of heptane concentration. The figure shows satisfactory agreement between the results obtained in this chapter and those from the literature for a temperature of $25{ }^{\circ} \mathrm{C}$. We also considered other temperatures and found excellent agreement with data by Korsching [80] for $35^{\circ} \mathrm{C}$ and with Ecenarro et al. [46] for $37.5^{\circ} \mathrm{C}$. At the same time our data for $35^{\circ} \mathrm{C}$ are $6 \%$ smaller than those of Bou-Ali et al. [20]. Soret coefficients for an equimolar heptane/benzene mixture at different temperatures agree fairly well with the data reported by Trevoy et al. [165]. Fig. 4.6 shows the transport coefficients $S_{\mathrm{T}}, D$, and $D_{T}$ for equimolar mixtures of benzene and isomers 


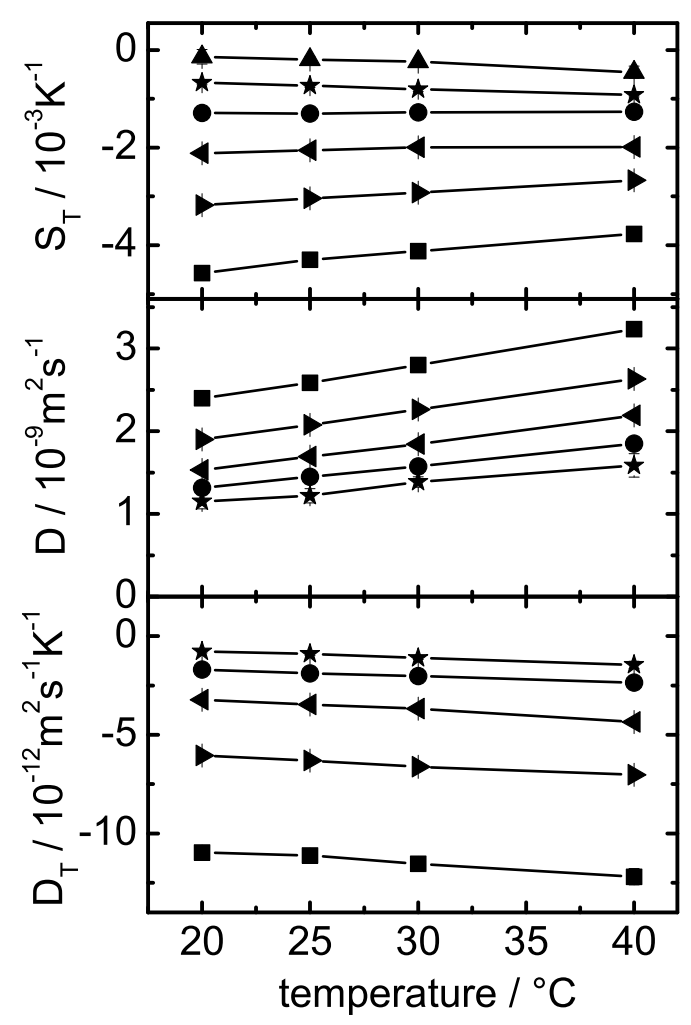

Figure 4.3: Transport coefficients for equimolar mixtures of benzene and linear alkanes as a function of temperature. The symbols indicate the Soret coefficients $S_{\mathrm{T}}$, diffusion coefficients $D$ and thermal diffusion coefficients $D_{\mathrm{T}}$ of heptane ( $\left.\mathbf{\square}\right)$, nonane ( $\bullet$ ), undecane ( $\left.\triangleleft\right)$, tridecane $(\bullet)$, and pentadecane $(\star)$ and the Soret coefficients of heptadecane $(\boldsymbol{\Delta})$ in benzene. The lines connect the data points. 


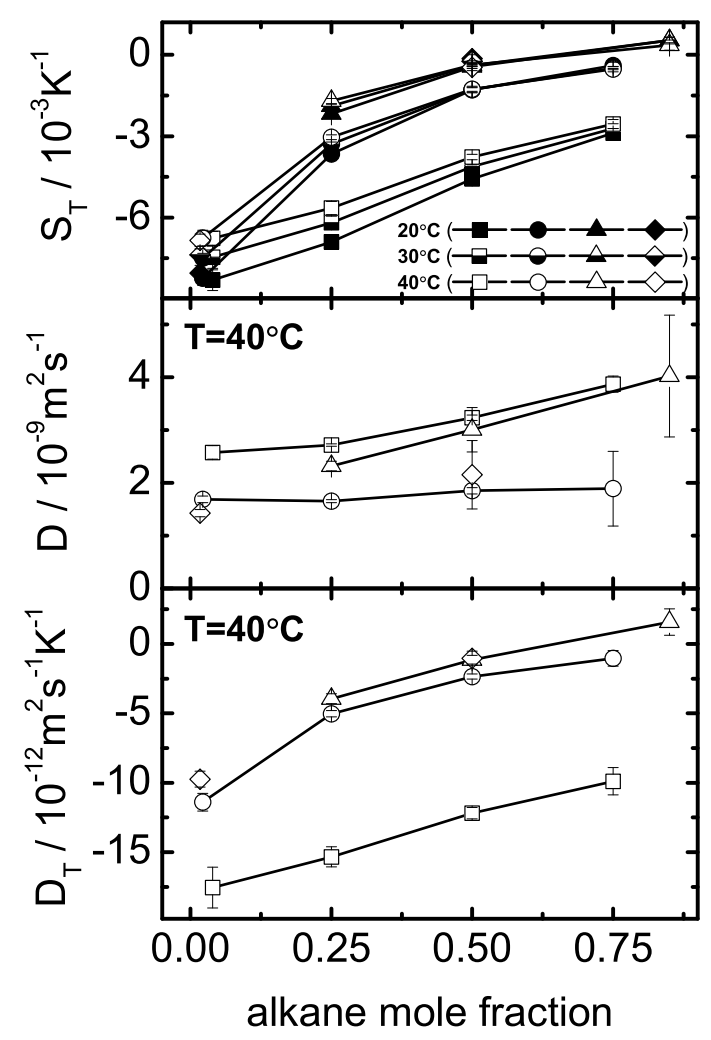

Figure 4.4: Soret coefficient $S_{\mathrm{T}}$, diffusion coefficient $D$ and thermal diffusion coefficient $D_{\mathrm{T}}$ of heptane (squares), tridecane (circles), heptadecane (diamonds), and 2,2,4-TMP (triangles) in benzene as a function of the alkane concentration. The top panel shows results for $S_{\mathrm{T}}$ at three different temperatures as indicated. The lower panels show results for $D$ and $D_{T}$ at a temperature of $40{ }^{\circ} \mathrm{C}$. The lines connect the data points. 


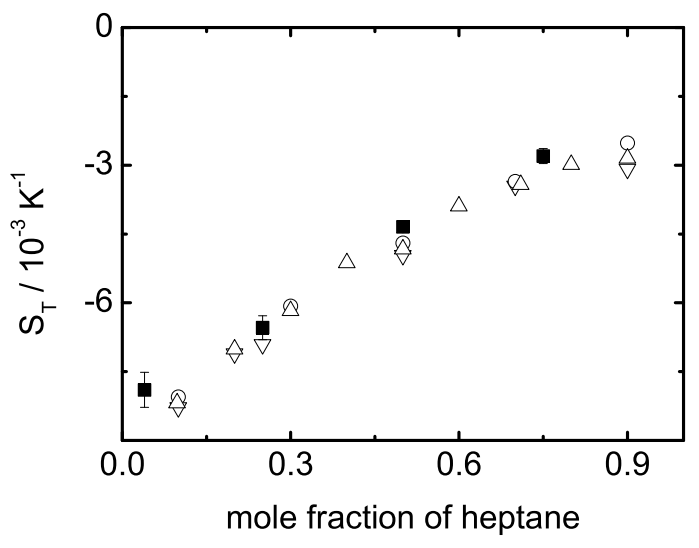

Figure 4.5: Soret coefficients of heptane/benzene mixtures at a temperature of $25^{\circ} \mathrm{C}$ as a function of heptane mole fraction. The symbols represent experimental data by (Bou-Ali et al. [20] $(\nabla)$, Korsching [81] (०), Demichowicz-Pigoniowa et al. [37] ( $\triangle$ ) and from TDFRS 
of heptane as a function of temperature. As in the case of the linear alkanes, the Soret coefficients are negative, except for the strongly branched 2,2,3-trimethylbutane (2,2,3-TMB), which has a positive Soret coefficient. At all temperatures, we find that the Soret coefficients increase with increasing number of side groups of the isomers. This implies that branching decreases the tendency for the alkanes to move to the warmer regions of a fluid. The diffusion coefficients of heptane and the isomers 2-MH, 3-MH and 2,4-DMP are the same within experimental uncertainty, while the diffusion coefficients are lower for the remaining isomers, 2,3-DMP and 2,2,3-TMB. Higher values of the viscosity may be responsible for the smaller values for the diffusion coefficient of 2,3-DMP and 2,2,3-TMB [186].

\subsection{Lattice model for the Soret effect in alkane/ben- zene mixtures}

In this chapter, we describe mixtures of benzene and normal alkanes with a simple lattice model that includes the effects of compressibility and orientation dependent interactions between benzene molecules. Consider a simple cubic lattice (coordination number $z=6$ ) with $N_{L}$ sites of which $N_{\mathrm{b}}$ and $N_{\mathrm{a}}$ are occupied by benzene and the alkane, respectively. In order to account for compressibility, some of the sites will be unoccupied so that $N_{L}=N_{\mathrm{b}}+N_{\mathrm{a}}+N_{\mathrm{v}}$, where $N_{\mathrm{v}}$ is the number of voids. The total volume of the lattice is $V=v N_{L}$, where $v$ is the volume of one elementary cube. Interactions between occupied nearest neighbor sites are described by interaction energies $\varepsilon_{i j}$, where the subscripts indicate the occupants of the sites ( $b$ for benzene and a for the alkane; voids are assumed to have zero interaction energies).

In order to account for the orientation dependence of interactions between benzene molecules in an approximate way, we introduce an orientational degree of freedom for the benzene sites on the lattice. A disk on a simple cubic lattice may be oriented so that its normal is aligned with the $x, y$, or $z$ axis. Accordingly, we assign one of three possible orientations to each site occupied by benzene. In order to distinguish between different relative orientations, we introduce two interaction energies for benzene-benzene interactions. The energy parameter $\varepsilon_{b b ; p}$ corresponds to preferred relative orientations of benzene molecules and is lower than the parameter $\varepsilon_{b b, n}$ for the remaining orientations. A fraction $f$ of the possible relative orientations is assumed to have the lower interaction energy. To estimate this fraction 


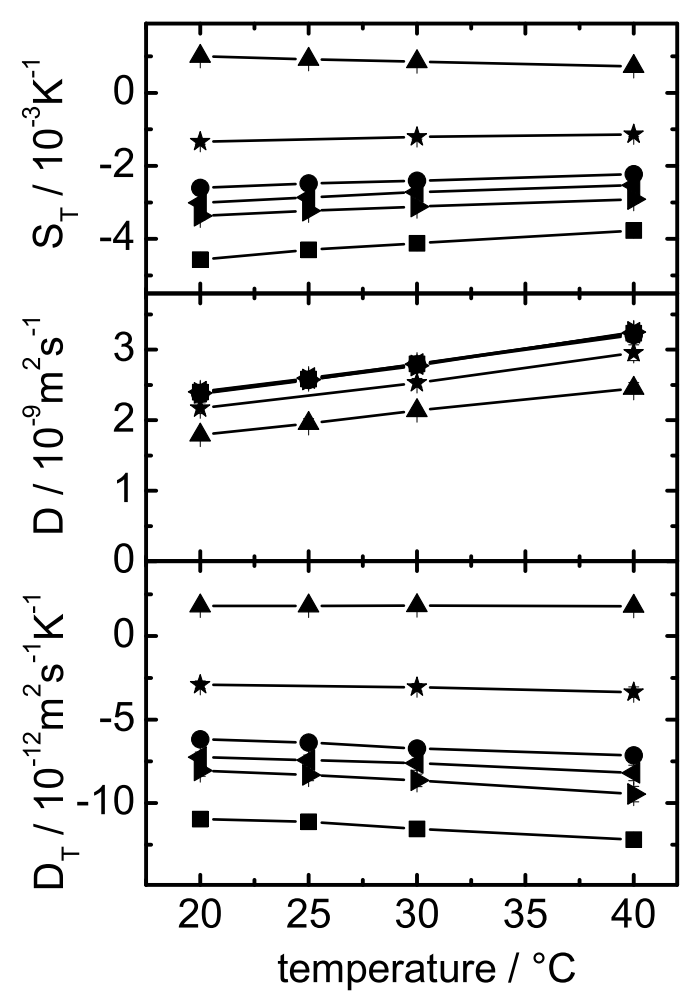

Figure 4.6: Soret coefficient $S_{\mathrm{T}}$, diffusion coefficient $D$ and thermal diffusion coefficient $D_{\mathrm{T}}$ of heptane $(\boldsymbol{\square}), 2-\mathrm{MH}(\bullet), 3-\mathrm{MH}(\triangleleft), 2,4-\mathrm{DMP}(\bullet), 2,3-\mathrm{DMP}(\star)$, and 2,2,3-TMB $(\boldsymbol{\Delta})$ in benzene at a mole fraction of 0.5 as a function of the temperature. The lines connect the data points. 
for our lattice model, consider a disk located at the origin (coordinates $(0,0,0)$ ) and aligned with the $z$-axis. If the nearest neighbor site $(0,0,1)$ or $(0,0,-1)$ is occupied by another disk, then two of the three possible orientations of this disk correspond to " $\mathrm{T}$ " configurations with interaction energy $\varepsilon_{b b ; p}$. For the nearest neighbor sites $( \pm 1,0,0)$ and $(0, \pm 1,0)$, on the other hand, only one of three orientations of the occupying disk yields a "T" configuration. Hence, eight of the total of eighteen relative orientations corresponds to favorable interactions, which yields $f=4 / 9$ for the fraction of preferred orientations.

A first approximation to the probability $p_{p}$ that two benzene sites make a preferred contact at a given temperature $T$ is given by

$$
p_{p}=\frac{f e^{-\beta \varepsilon_{b b ; p}}}{f e^{-\beta \varepsilon_{b b ; p}}+(1-f) e^{-\beta \varepsilon_{b b ; n}}},
$$

where $\beta=1 / k_{B} T$ and $k_{B}$ is Boltzmann's constant. With a random mixing approximation [84] for the arrangement of the different sites on the lattice, we obtain for the internal energy per site, $u$,

$$
u=\frac{z}{2}\left(\phi_{b}^{2}\left[p_{p} \varepsilon_{b b ; p}+\left(1-p_{p}\right) \varepsilon_{b b ; n}\right]+\phi_{a}^{2} \varepsilon_{a a}+\phi_{a} \phi_{b} \varepsilon_{a b}\right)
$$

where the energy parameters $\varepsilon_{a a}$ and $\varepsilon_{a b}$ describe alkane-alkane and alkane-benzene interactions, respectively, and where $\phi_{a}, \phi_{b}$, and $\phi_{v}$ denote the fractions of lattice sites occupied by the alkane, benzene, and voids, respectively. With these approximations, the canonical partition function of the system may be written as

$$
\begin{gathered}
Z\left(N_{L}, T, N_{a}, N_{b}\right)=3^{N_{b}}\left(\begin{array}{c}
N_{L} \\
N_{a}
\end{array}\right)\left(\begin{array}{c}
N_{L}-N_{a} \\
N_{b}
\end{array}\right) \\
\quad \times \exp \left\{-\beta N_{L} \frac{z}{2}\left(\phi_{b}^{2} \tilde{\varepsilon}_{b b}+\phi_{a}^{2} \varepsilon_{a a}+\phi_{a} \phi_{b} \varepsilon_{a b}\right)\right\},
\end{gathered}
$$

where an effective interaction parameter $\tilde{\varepsilon}_{b b}$ has been introduced,

$$
e^{-\beta \tilde{\varepsilon}_{b b}}=f e^{-\beta \varepsilon_{b b ; p}}+(1-f) e^{-\beta \varepsilon_{b b ; n}}
$$

From the partition function, the pressure of the system may be calculated according to

$$
P=\frac{1}{\beta v}\left(\frac{\partial Z}{\partial N_{L}}\right)_{N_{a}, N_{b}} .
$$


In Appendix 4.6 we describe how the system-dependent parameters for the alkane/benzene mixtures were determined. In the following, we use a lattice with $N_{L}=5000$ sites. For a given temperature, pressure, and composition of a mixture, the occupation numbers $N_{a}$ and $N_{b}$ are determined by gradually filling the lattice with alkane and benzene sites in the right proportion until the pressure reaches the desired value. Due to the discrete nature of the lattice, the targeted pressure cannot be reached exactly. However, the size of the lattice ensures that the deviations from the target pressure have a negligible effect on the results presented here.

\subsubsection{Calculation of Soret coefficients}

In order to describe thermodiffusion, we divide the lattice into two equal chambers, $\mathrm{A}$ and B, each with lattice sites $N^{A}=N^{B}=N_{L} / 2$ but with slightly different temperatures, $T^{A}=$ $T^{B}+\delta T$. With $N_{a}$ and $N_{b}$ denoting the alkane and benzene occupation numbers of the whole lattice, chambers $\mathrm{A}$ and $\mathrm{B}$ have occupation numbers $\left\{N_{a}^{A}, N_{b}^{A}\right\}$ and $\left\{N_{a}^{B}=N_{a}-N_{a}^{A}, N_{b}^{B}=\right.$ $\left.N_{b}-N_{b}^{A}\right\}$, respectively. Under the assumption that the chambers are non-interacting, the partition function of the whole system is the product of the the partition functions of the chambers, $Z^{A} Z^{B}$. A sum of states $Q$ may then be defined by summing over all possible occupations of the two lattices [90]

$$
\begin{aligned}
Q=\sum_{\left[N_{a}^{A}, N_{b}^{A}\right]} & Z^{A}\left(N^{A}, T, N_{a}^{A}, N_{b}^{A}\right) \\
& \quad \times Z^{B}\left(N^{B}, T, N_{a}-N_{a}^{A}, N_{b}-N_{b}^{A}\right),
\end{aligned}
$$

where the square brackets indicate a summation consistent with the total number of particles and lattice sites.

We evaluate Eq. (4.9) by exact enumeration of all possible occupations of the chambers. As we are performing the calculation of the terms in the sum of states, we also calculate the mass fractions of the alkane, $w^{A}\left(N_{a}^{A}, N_{b}^{A}\right)$ and $w^{B}\left(N_{a}-N_{a}^{A}, N_{b}-N_{b}^{A}\right)$ using Eq. (4.15) applied to the filling fraction of the chambers. The average mass fraction $\bar{w}^{A}$ of the alkane in chamber A is determined by the weighted sum

$$
\begin{gathered}
\bar{w}^{A}=\frac{1}{Q} \sum_{\left[N_{a}^{A}, N_{b}^{A}\right]} w^{A}\left(N_{a}^{A}, N_{b}^{A}\right) Z^{A}\left(N^{A}, T, N_{a}^{A}, N_{b}^{A}\right) \\
\times Z^{B}\left(N^{B}, T, N_{a}-N_{a}^{A}, N_{b}-N_{b}^{A}\right),
\end{gathered}
$$


and similarly for the average mass fraction $\bar{w}^{B}$ in chamber B. Finally, the Soret coefficient of the alkane is calculated from

$$
S_{\mathrm{T}}=-\frac{1}{w(1-w)} \frac{\bar{w}^{A}-\bar{w}^{B}}{T^{A}-T^{B}},
$$

where $w$ is the alkane mass fraction of the whole system.

\subsubsection{Comparison with experimental data}

In Fig. 4.7 we present predictions from our lattice model for the Soret coefficients of equimolar $n$-alkane/benzene mixtures as a function of temperature. In agreement with the experimental data shown in Fig. 4.3, the $S_{\mathrm{T}}$ values calculated from Eq. (4.11) increase with increasing chain length. As in the case of the experimental data, the slope of the Soret coefficients as a function of temperature decreases with increasing chain length. However, at this composition, the predicted slope of $S_{\mathrm{T}}$ versus $T$ is positive for all chain lengths whereas the experiments show a negative slope for the longest chains. The sign of the slope is composition dependent. For low alkane concentrations, both theory and experiment show positive slopes for all chain lengths. As the alkane content increases, the slope decreases and becomes negative for the longest chains at high alkane concentration. For tridecane, for example, the experimental data presented in Fig. 4.4 show the Soret coefficient to increase with temperature for $x=0.25$, to be almost independent of temperature for $x=0.5$, and to decrease with temperature for $x=0.75$. The calculated $S_{\mathrm{T}}$ values for tridecane change from increasing with temperature to decreasing with temperature at a higher alkane content $(x \simeq 0.92)$ and only after the calculated Soret coefficients have become positive. For heptadecane, the change in behavior in the experimental data occurs for a concentration smaller than $x=0.5$ while the calculated values change behavior near $x \simeq 0.78$. Fig. 4.8 shows Soret coefficients as a function of chain length $N$ of the alkanes at a fixed temperature of $30^{\circ} \mathrm{C}$ for the same mixtures as in Fig. 4.7. A comparison between theory (open symbols) and experiment (filled symbols) shows that the model describes well the trend in the chain length dependence but that the calculated $S_{\mathrm{T}}$ values are always between 0.5 and $1.3 \times 10^{-3} \mathrm{~K}^{-1}$ higher than the experimental values at this composition. In Fig. 4.9 we present Soret coefficients of heptane and tridecane in mixtures with benzene as a function of composition for three different temperatures. The symbols connected by dashed lines represent experimental data (see also Fig. 4.4) while the 


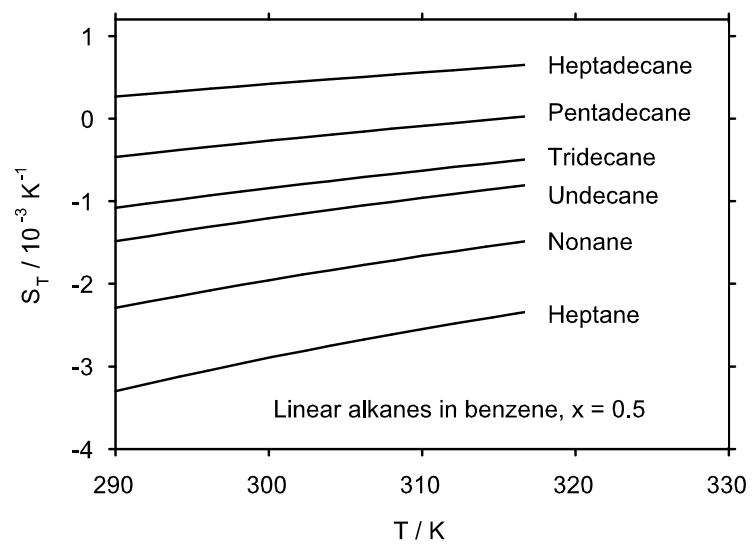

Figure 4.7: Alkane Soret coefficients as a function of temperature for mixtures of benzene and linear alkanes at a mol fraction of $x=0.5$ and atmospheric pressure. The solid lines indicate values for the Soret coefficient $S_{\mathrm{T}}$ calculated from Eq. (4.11) as described in the text.

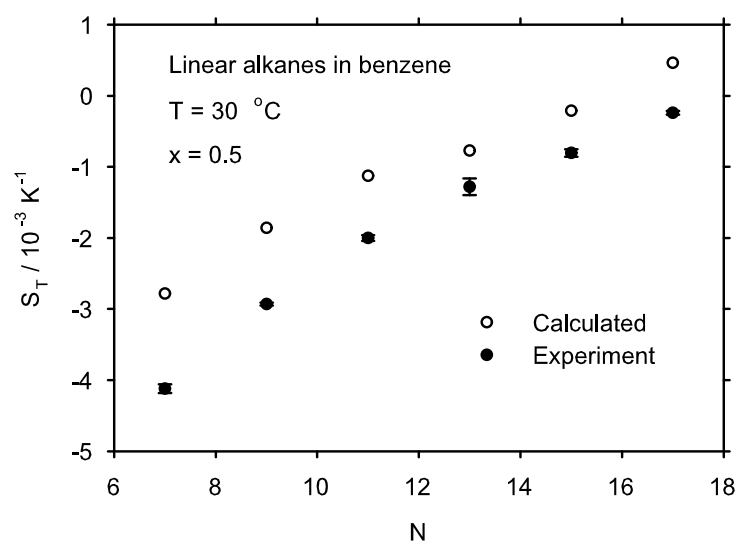

Figure 4.8: Alkane Soret coefficients as a function of number of carbon atoms in the alkane chain for mixtures of benzene and linear alkanes at a mol fraction of $x=0.5$ and a temperature of $30{ }^{\circ} \mathrm{C}$. The open symbols represent values for the Soret coefficient calculated from Eq. (4.11), the filled symbols represent experimental results from TDFRS. 


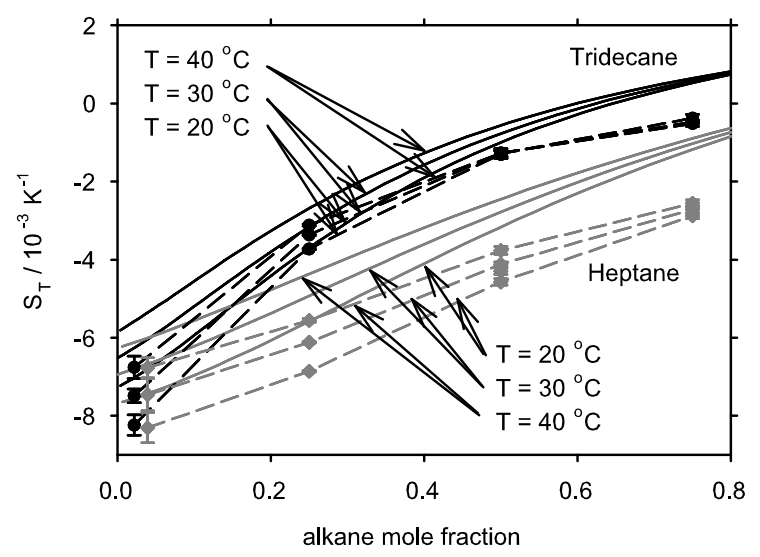

Figure 4.9: Soret coefficients of heptane and tridecane in mixtures with benzene as a function of composition for three different temperatures. The solid lines indicate values for the Soret coefficient calculated from Eq. (4.11), the symbols represent experimental results from TDFRS (see Fig. 4.4), the dashed lines connect data points.

solid lines represent Soret coefficients calculated from Eq. (4.11). Both theory and experiment show an increase of the alkane Soret coefficients with increasing alkane mole fraction, $x$. They also show that the temperature dependence of the Soret effect is larger for benzenerich mixtures than for alkane rich mixtures, as discussed above. As in the comparison at fixed mixture composition, the theory reproduces the trends in the experimental data but it overestimates the values of the Soret coefficients. Experimental data are most easily interpreted for mixtures where the alkane concentration is very low. For dilute alkane mixtures, the density of the mixture is close to that of pure benzene. In addition, each alkane molecule is surrounded by benzene molecules so that interactions between alkane molecules do not play a role. For dilute solutions, our lattice model shows a close correlation between (net) interaction parameters and the Soret coefficient: the minority component is enriched in the warmer regions of the fluid (and its Soret coefficient is negative) when the contribution to the internal energy of the mixed interaction is less negative (less attractive) than that of the interactions between molecules of the majority component. The absolute value of the Soret coefficient decreases as the magnitude of the difference between contributions from mixed 
and like interactions decreases. For mixtures dilute in alkane, the relevant interaction energy for like interactions is the average energy associated with a benzene-benzene contact

$$
\left\langle\varepsilon_{b b}\right\rangle=p_{p} \varepsilon_{b b ; p}+\left(1-p_{p}\right) \varepsilon_{b b ; n}
$$

where the probability $p_{p}$ is given by Eq. (4.4).

Figure 4.10 shows temperature dependent Soret coefficients for mixtures with very low alkane mass fractions $(w=0.05)$. The symbols represent experimental data (see Fig. 4.4) for heptadecane, tridecane, and heptane. The solid lines show calculated values for heptane, tridecane, and heptadecane, where the curves for tridecane and heptadecane are indistinguishable in this graph. The experiments on $w=0.05$ mixtures yield negative Soret coefficients for the alkanes (heptane, tridecane, and heptadecane) with values that are independent of chain length within the uncertainty of the experiment. The observed chain-length independence may be due to a competition between two effects. For dilute solutions of chain molecules, one often observes thermal diffusion coefficients that are chain-length independent [76, 179, 26] and, since mutual diffusion coefficients decrease with increasing chain length, Soret coefficients whose absolute values increase with molecular mass. For the $n$-alkane/benzene mixtures at higher concentrations, on the other hand, we observe absolute values of the Soret coefficients that decrease with chain length. Additional measurements at lower and intermediate concentrations would be required to test if this competition between effects indeed takes place. The predicted values of the Soret coefficients in Fig. 4.10 are negative and systematically higher than the experimental values. The calculated $S_{\mathrm{T}}$ values, however, show a chain length dependence for shorter chains and become independent of $N$ when the mixed benzene-alkane interactions become chain-length independent, that is for $N=11$ to $N=17$ (see Table 4.1). In the present lattice model, there is no chain connectivity and no distinction between alkane sites at chain ends (methyl groups) and along the chain (ethyl groups). The differences between theoretical and experimental values suggest that such a distinction is important.

Fig. 4.10 shows that our model reproduces the temperature dependence of the Soret coefficients for mixtures with low alkane content very well. In order to investigate the role of thermal expansion, we also performed constant density calculations for these mixtures. The inset shows as dashed lines calculated $S_{\mathrm{T}}$ values as a function of temperature for a constant density corresponding to the density at atmospheric pressure for the lowest temperature dis- 
played. A comparison of the results from the constant pressure (solid lines) and constant density (dashed lines) calculations shows that thermal expansion of the mixtures with low alkane content leads to more negative values of the Soret coefficients for the alkanes. At constant density, the calculated temperature dependence of the Soret coefficients is a consequence of the temperature dependence of the average energy per benzene-benzene contact, Eq. (4.12). The temperature dependence at constant pressure also includes a contribution due to the density decrease with increasing temperature. For mixtures that are rich in benzene, a decrease in density leads to the disruption of mixed benzene-alkane contacts and has a similar effect as a decrease in the magnitude of the mixed interaction energy, i.e. an increase in the tendency of the alkane to move to the warmer regions of the fluid. The concentration dependent Soret coefficients displayed in Figs. 4.4 and 4.9 show that the tendency of alkane molecules to migrate to the warmer regions of the fluid decreases (the Soret coefficients become more positive) with increasing alkane concentration for all temperatures and alkane molecules investigated. When the concentrations of alkane and benzene molecules are nearly equal, close to a weight fraction of $w=0.5$, the differences in the interactions between like molecules dominate the Soret effect. Since benzene-benzene interactions are more attractive than alkane-alkane interactions, we observe negative Soret coefficients for all alkanes. (Please note that the values in Fig. 4.7 are calculated for a mole fraction of $x=0.5$ which corresponds to a mass fraction of about $w=0.75$ for heptadecane). Part of the concentration dependence of the Soret coefficients is due to the difference in interaction energies for benzene-alkane and alkane-alkane contacts. The parameters in Table 4.1 show that the mixed interactions $\varepsilon_{a b}$ are less attractive than the alkane-alkane interactions $\varepsilon_{a a}$. Since the number of alkane-alkane contacts increases with increasing alkane concentration, and since the difference between alkane-alkane and benzene-benzene interactions is smaller than that between mixed interactions and benzene-benzene interactions, the Soret coefficient is expected to become more positive with increasing alkane content. In addition, the density decreases with increasing alkane composition and leads to a further increase of the alkane Soret coefficient. For high alkane concentrations, finally, the Soret effect is dominated by the difference in benzene-alkane and alkane-alkane interactions. While the TDFRS results suggest that the Soret coefficient of tridecane remains negative as the mixture becomes dilute in benzene, our calculations yield positive Soret coefficients for tridecane at high alkane content. This shows 


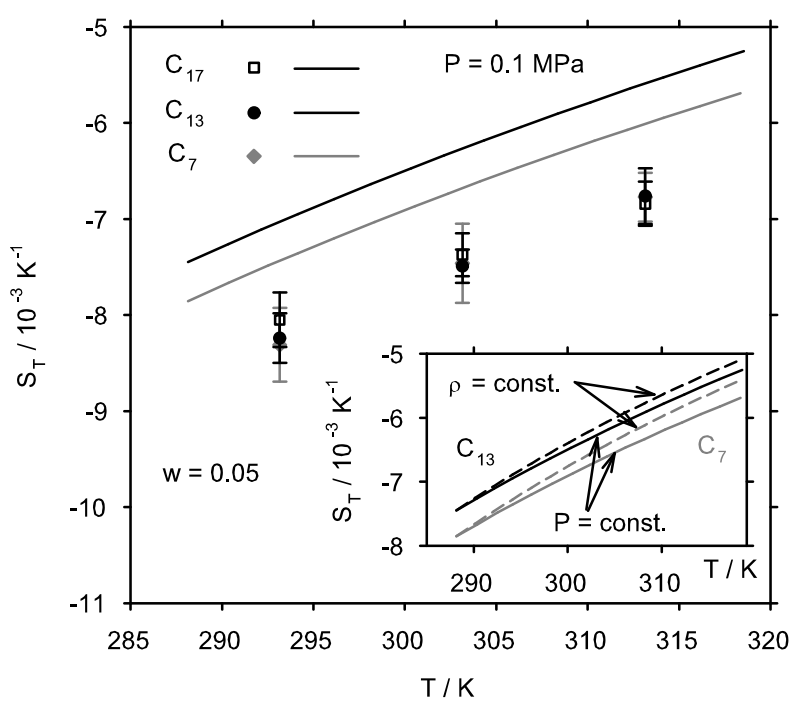

Figure 4.10: Alkane Soret coefficients as a function of temperature for mixtures of benzene and linear alkanes at a mass fraction of $w=0.05$ and atmospheric pressure. The symbols represent experimental data for heptadecane $\left(\mathrm{C}_{17}\right)$, tridecane $\left(\mathrm{C}_{13}\right)$, and heptane $\left(\mathrm{C}_{7}\right)$. The solid lines represent values for the Soret coefficient $S_{\mathrm{T}}$ calculated from Eq. (4.11) for a pressure of $0.1 \mathrm{MPa}$ for heptane (gray), tridecane (black) and heptadecane (indistinguishable from tridecane in the graph). The inset compares calculations at constant pressure, $P=0.1 \mathrm{MPa}$, (solid lines) with calculations at constant density (dashed lines), $\rho=869.2 \mathrm{~kg} / \mathrm{m}^{3}$ for heptane and $\rho=874.0 \mathrm{~kg} / \mathrm{m}^{3}$ for tridecane. 
that the effective benzene-alkane interaction at high alkane content is more attractive than described by our simple lattice model.

In summary, the comparison of the predictions from our two-chamber lattice model with the experimental values for the Soret coefficients shows that the model captures the trends of the variation of the Soret coefficient with temperature, molecular mass and composition. The differences between calculated and experimental values are most pronounced for alkane-rich mixtures. There are several reasons for the increase in the deviations between theory and experiment with increasing alkane content. For one, the random mixing approximation that we have employed in our lattice model is expected to be most appropriate for mixtures with low alkane concentrations since the concentration of both voids and alkane sites is low for these mixtures. As the alkane content increases at constant pressure, the total filling fraction decreases (with the density) and differences in the interaction energies are expected to lead to non-random distributions of nearest neighbor contacts. Furthermore, the lattice model for the alkanes is very simple and the determination of system-dependent parameters started from benzene, allowing only two adjustable parameters to describe the thermodynamics of the pure alkane fluids. A more sophisticated model for the alkanes that allows, for example, for different interaction energies for chain ends, sites along a linear chain, and branch points should lead to better description of the Soret effect for alkane rich mixtures and also allow us to investigate mixtures of branched alkane isomers. Unfortunately, the available thermodynamic data for the mixture systems investigated here are not sufficiently detailed to determine the system-dependent parameters for models with several interaction parameters for alkanealkane and alkane-benzene interactions. However, our simple lattice model helps us separate molecular mass and density effects from effects due to differences in molecular interactions.

\subsection{Discussion and Conclusion}

The thermal diffusion forced Raleigh scattering experiments have shown that the Soret effect in alkane/benzene mixtures depends on the molecular mass and structure of the alkane as well as the temperature and composition of the mixture. For the linear chains, a simple lattice model is able to reproduce the experimental trends. For the branched isomers, however, an interpretation of the data is more difficult. A comparison of the experimental data for 
branched heptane isomers in Fig. 4.6 and with those for the linear chains between heptane and heptadecane in Fig. 4.3 shows that the effect of branching on the Soret coefficients is larger than that of the molecular weight. This is not expected from the thermodynamic properties of the pure alkane fluids; the density at a given temperature, for example, depends much more strongly on the chain length than on the molecular architecture.

The moments of inertia have been shown to make an important contribution to the Soret effect for mixtures of cyclohexane and benzene isotopes [36], see Eq. (4.2). In order to explore this effect we have calculated the moments of inertia about the symmetry axis $\left(I_{z z}\right.$ for the disk-like benzene molecules, $I_{x x}$ for the more prolate alkane molecules) using an atomistic model for single molecules in vacuum [5]. In Fig. 4.11 we present the measured Soret coefficients of the linear and branched alkanes at a temperature of $20^{\circ} \mathrm{C}$ and a mole fraction of $x=0.5$ as a function of the difference $\delta_{I}=I_{x x \text {,alkane }}-I_{z z \text {,benzene. For the linear }}$ alkanes, there is an almost linear correlation between $\delta_{I}$ and the Soret coefficients. This is not unexpected since $I_{x x}$,alkane grows almost linearly with chain length as do the Soret coefficients, which makes it difficult to separate the mass and moment of inertia contributions in Eq. (4.2). However, in contrast to what was found for the isotope mixtures, the coefficients in Eq. (4.2) representing the slope of the $S_{\mathrm{T}}$ values are not independent of composition. This suggests that a separation into mass, moment of inertia, and chemical contributions is not straightforward for the alkane mixtures.

For the branched alkanes, the moments of inertia $I_{x x \text {,alkane increase with increasing number }}$ of methyl groups much as the Soret coefficients. On the other hand, the chemical contribution is also expected to change with alkane architecture. When comparing theory and experiment for linear alkane mixtures with low alkane mass fraction $(w=0.05)$ we noted that the experimental data suggested different interaction energies between benzene molecules and methyl groups and benzene molecules and ethyl groups. Similar differences are expected to hold for interactions between different carbon groups of branched alkane molecules [133, 27] and between such groups and benzene molecules. 


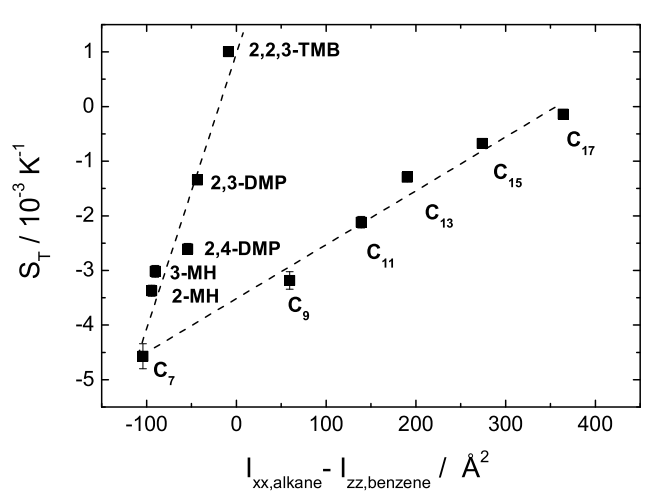

Figure 4.11: Soret coefficients of heptane isomers and linear alkanes as function of the difference in principal moments of inertia as discussed in the text. The symbols represent experimental values for the Soret coefficients at a temperature of $20^{\circ} \mathrm{C}$ and a mole fraction of $x=0.5$. The dashed lines are a guide to the eye.

\subsection{Appendix: Determination of system-dependent pa- rameters for alkane/benzene mixtures}

Our lattice model for alkane/benzene mixtures has seven system-dependent parameters. In order to determine these parameters, we consider the thermodynamic limit of the partition function introduced in Eq. (4.6) of Section 4.4. In this limit, Eq. (4.8) for the pressure of the mixtures yields

$$
P=-\frac{1}{\beta v}\left[\ln \left(1-\phi_{v}\right)-\frac{z}{2} \beta\left(\phi_{b}^{2} \tilde{\varepsilon}_{b b}+\phi_{a}^{2} \varepsilon_{a}+\phi_{a} \phi_{b} \varepsilon_{a b}\right)\right],
$$

The filling fractions are related to the density and composition of the mixture through

$$
\phi_{a}=v r_{a} x \tilde{\rho}, \quad \phi_{b}=v r_{b}(1-x) \tilde{\rho}, \quad \phi_{v}=1-\phi_{a}-\phi_{b},
$$

where $x$ is the mol fraction of the alkane and $\tilde{\rho}$ is the number density of the mixture. Here, $v$ is the volume per lattice site and the numbers $r_{a}$ and $r_{b}$ represent the number of lattice sites per alkane and benzene molecule, respectively. The mass fraction $w$ of the alkane is related 
Table 4.1: System-dependent parameters for alkane/benzene mixtures; $M_{w, a}$ and $M_{w, b}$ denote the molecular masses, $r_{a}$ and $r_{b}$ the number of lattice sites per alkane and benzene molecule, respectively, $\varepsilon_{i j}$ are interaction parameters as discussed in the text, and $f$ is the fraction of favorable benzene-benzene contacts.

\begin{tabular}{l|r|c|c|c} 
Lattice site volume $v=2.2348 \times 10^{-5} \mathrm{~m}^{3} \cdot \mathrm{mol}^{-1}$ \\
\hline $\begin{array}{c}\mathrm{M}_{w, b} \\
\mathrm{~g} \cdot \mathrm{mol}^{-1}\end{array}$ & $r_{b}$ & $\begin{array}{c}\varepsilon_{b b ; p} \\
\mathrm{~J} \cdot \mathrm{mol}^{-1}\end{array}$ & $\begin{array}{c}\varepsilon_{b b ; n} \\
\mathrm{~J} \cdot \mathrm{mol}^{-1}\end{array}$ \\
\hline$f=0.444$ & 78.11 & 3.7870 & -4142.4 & -226.87 \\
\hline & $\mathrm{M}_{w, a}$ & $r_{a}$ & $\varepsilon_{a a}$ & $\varepsilon_{a b}$ \\
Alkane & $\mathrm{g} \cdot \mathrm{mol}^{-1}$ & & $\mathrm{~J} \cdot \mathrm{mol}^{-1}$ & $\mathrm{~J} \cdot \mathrm{mol}^{-1}$ \\
\hline Heptane & 100.21 & 6.1064 & -2526.9 & -2506 \\
Nonane & 128.26 & 7.5131 & -2596.4 & -2526 \\
Undecane & 156.31 & 8.9359 & -2648.7 & -2539 \\
Tridecane & 184.37 & 10.311 & -2659.4 & -2540 \\
Pentadecane & 212.42 & 11.755 & -2696.8 & -2543 \\
Heptadecane & 240.48 & 13.225 & -2743.0 & -2540 \\
\hline
\end{tabular}

to the filling fractions through

$$
w=\frac{\phi_{a} M_{w, a} / r_{a}}{\phi_{a} M_{w, a} / r_{a}+\phi_{b} M_{w, b} / r_{b}},
$$

where $M_{w, a}$ and $M_{w, b}$ are the molecular masses of the alkane and benzene, respectively. In Eq. (4.13), $\varepsilon_{a a}$ and $\varepsilon_{a b}$ are the interaction parameters for alkane-alkane and alkane-benzene interactions, respectively. The effective parameter $\tilde{\varepsilon}_{b b}$ for benzene-benzene interactions was defined in Eq. (4.7) as $\exp \left(-\beta \tilde{\varepsilon}_{b b}\right)=f \exp \left(-\beta \varepsilon_{b b ; p}\right)+(1-f) \exp \left(-\beta \varepsilon_{b b ; n}\right)$, where $f=4 / 9$ is the fraction of preferred contacts and $\varepsilon_{b b ; p}$ and $\varepsilon_{b b ; n}$ represent the interaction energies for preferred and non-preferred contacts, respectively. For the one-component liquid benzene, we determined the system-dependent parameters $v, r_{b}, \varepsilon_{b b ; p}$ and $\varepsilon_{b b ; n}$ from a comparison of calculated values of thermophysical properties with tabulated values from the NIST Chemistry WebBook [4] based on the equation of state by Polt et al. [119]. In the temperature range between 288 and $318 \mathrm{~K}$ we considered saturated liquid densities, densities at the constant 


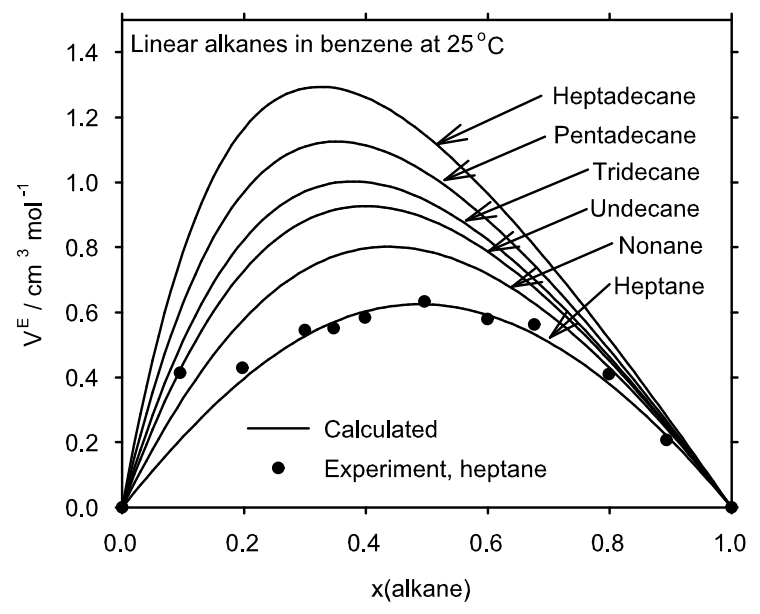

Figure 4.12: Excess volume of alkane/benzene mixtures at a temperature of $25{ }^{\circ} \mathrm{C}$ and atmospheric pressure. The symbols represent experimental data for heptane/benzene mixtures by Kouris and Panayiotou [82], the lines represent values calculated from our lattice model with the parameters in Table 4.1 .

pressure of $P=0.1 \mathrm{MPa}$, and, also at $P=0.1 \mathrm{MPa}$, the combination of pressure derivatives $c_{p}-c_{v}=\tilde{\rho}^{-2} T(\partial P / \partial T)_{\tilde{\rho}}^{2} /(\partial P / \partial \tilde{\rho})_{T}$, where $c_{p}$ and $c_{v}$ are the molar isobaric and isochoric heat capacities, respectively. The resulting values for the system-dependent parameters are presented in Table 4.1. They correspond to a good representation of the saturated liquid densities (root mean squared relative deviation $(\mathrm{rmsd}) \approx 0.5 \%$, maximum deviation $-0.78 \%$ ), a very good representation of the densities at atmospheric pressure $(\mathrm{rmsd} \approx 0.1 \%$, maximum deviation $0.26 \%$, and a reasonable representation of $c_{p}-c_{v}(\mathrm{rmsd} \approx 11 \%$, maximum deviation $-19 \%)$. The value of the volume per lattice site $v$ obtained for benzene, was also adopted for the pure alkanes and for all mixtures. For each of the alkanes, the two remaining system-dependent parameters $r_{a}$ and $\varepsilon_{a a}$ were determined from a comparison with tabulated values for the density at atmospheric pressure $[32,186]$ and the corresponding thermal expansion coefficients [186], $\alpha=\tilde{\rho}^{-1}(\partial P / \partial T)_{\tilde{\rho}} /(\partial P / \partial \tilde{\rho})_{T}$, in the temperature range from 288 to $318 \mathrm{~K}$. The resulting values for the parameters $r_{a}$ and $\varepsilon_{a a}$ are included in Table 4.1. They correspond to a very good representation of the densities (maximum deviation $-0.12 \%$ ) 
and a reasonable representation of the thermal expansion coefficients ( $\mathrm{rmsd} \approx 9-10 \%)$.

Finally, in order to establish values for the mixed interaction energies $\varepsilon_{a b}$, we consider the excess molar volume $V^{E}$ of alkane/benzene mixtures

$$
V^{E}=\frac{1}{\tilde{\rho}}-\frac{x}{\tilde{\rho}_{a}}-\frac{1-x}{\tilde{\rho}_{b}},
$$

where $\tilde{\rho}, \tilde{\rho}_{a}$, and $\tilde{\rho}_{b}$ are the molar densities of the mixture, the alkane, and benzene, respectively, at the given temperature and pressure. Kouris and Panayiotou [82] measured the density of benzene, heptane, and their mixtures at a temperature of $25^{\circ} \mathrm{C}$ and atmospheric pressure for a range of compositions. We determined a value for $\varepsilon_{a b}$ for heptane/benzene mixtures by comparing values for the excess volume derived from the experimental data with values calculated from our lattice model. In Fig. 4.12 we present excess volumes at $25^{\circ} \mathrm{C}$ and atmospheric pressure for the mixtures of benzene with linear alkanes considered in this chapter. The symbols and the lowest solid line represent experimental [82] and calculated values for the excess volume of heptane/benzene mixtures, respectively, and show that the simple lattice model reproduces the volume change on mixing well. Unfortunately, excess volume data are not available for the other mixtures of benzene and $n$-alkanes considered in this chapter. For mixtures of benzene with the even $n$-alkanes hexane through hexadecane, Awwad et al. [10] determined excess volumes at a temperature of $25^{\circ} \mathrm{C}$ and atmospheric pressure. We estimated values for the mixed interaction energies for the odd $n$-alkanes nonane through heptadecane, by requiring that the excess volumes be an increasing function of the chain length. The resulting values for the mixed interaction parameters are included in Table 4.1, the solid lines in Fig. 4.12 represent values calculated with these parameters. 


\section{Mixtures of branched heptane in benzene: RNEMD simulations}

We studied the thermal diffusion behavior of mixtures of benzene and heptane isomers by reverse nonequilibrium molecular dynamics. For $n$-heptane/benzene mixtures we investigated the concentration dependence of the Soret coefficient. The Soret coefficient for equimolar mixtures of the three heptane isomers 3methylhexane, 2,3-dimethylpentane and 2,4-dimethylpentane in benzene has been calculated. Compared to the experimental data, the simulation results show the same trend in dependence of the mole fraction and degree of branching. The negative Soret coefficient indicates the enrichment of alkanes in the warm side. In the case of the heptane isomers in benzene we could study the influence of the difference in shape and size on the thermal diffusion behavior at constant mass. In the simulation as well as in the experiment we found that the Soret coefficients becomes higher with increasing degree of branching. Such behavior can not be explained only by mass and size effects. The effect of the molecular shape needs to be considered additionally. *

\subsection{Introduction}

Thermal diffusion describes the migration of molecules in a temperature gradient. In the simple case of a binary mixture with constant pressure there is a mass diffusion current

$$
j_{\mathrm{D}}=-\rho D \nabla x
$$

* The work described in this chapter is accepted for publication in J. Chem. Phys. 
and a thermal diffusion current $j_{\mathrm{T}}=-\rho D_{\mathrm{T}} x(1-x) \nabla T$, with $x$ the molar fraction, $\rho$ the density of the liquid, and $D$ and $D_{\mathrm{T}}$ the mutual mass and thermal diffusion coefficients, respectively. In the stationary state the two flows cancel and the resulting concentration gradient is given by

$$
\nabla x=-S_{\mathrm{T}} x(1-x) \nabla T .
$$

$S_{\mathrm{T}}=D_{\mathrm{T}} / D$ is the Soret coefficient. A positive Soret coefficient of the component with the mole fraction $x$ implies that this component moves to the cold region of the fluid. The main practical applications are separation processes [145, 29] such as thermal field flow fractionation of polymers and colloids or isotope separation, characterization of geochemical processes [66, 30] and combustion [128].

The reverse nonequilibrium molecular dynamics (RNEMD) method has been developed to calculate the Soret coefficient. The energy flux is fixed by the boundary conditions and $S_{\mathrm{T}}$ can be easily calculated from temperature and concentration gradients according to Eq. 5.2. This method has been successfully applied for investigation of the thermal diffusion behavior in Lennard-Jones fluids [126], methane in "super" methane [56], methane/ $n$-decane [156], methane/n-alkane [55], $n$-pentane/n-decane [113], benzene/cyclohexane [189] and water/alcohol [104] mixtures. In the Chapter 2 we also investigated the thermal diffusion process in binary mixtures of simple molecules (tetraethylsilane, di-tert-buthylsilane and carbon tetrabromide in carbon tetrachloride) by thermal diffusion forced Rayleigh scattering (TDFRS) and MD simulations. It was found, that the component with the larger mass and larger Hildebrandt parameter moves to the cold side. This is the typical behavior of ideal solutions of spherical molecules, for which the enthalpy of mixing and the mixing volume are close to zero. The Hildebrandt parameter provides a numerical estimate of the degree of interaction between materials, and can be a good indication of solubility. For organic compounds it can be estimated according to $\delta=\sqrt{\rho\left(H^{\mathrm{vap}}-R T\right) / M}$ with the gas constant $\mathrm{R}$ and enthalpy of vaporization $H^{\text {vap }}$.

However, this simple rule of thumb fails for alkane/benzene mixtures (c.f. Chapter 4). The heavier linear alkane always moves to the warm side. This tendency becomes weaker with increasing degree of branching and the highly branched isomer of heptane (2,2,3 - TMB) moves to the cold side. The thermal diffusion behavior of linear alkanes is well described by a simple lattice model (SLM) (c.f. Chapter 4). At the same time the SLM is not capable 
to describe the thermal diffusion behavior of branched alkanes because their thermodynamic parameters such as density, heat capacity and thermal expansion coefficient are not sensitive to the degree of branching. In this chapter we investigated the dependence of the transport properties versus concentration for $n$-heptane/benzene mixture and versus the degree of branching for equimolar branched heptane/benzene mixtures by RNEMD simulation. The obtained simulation results have been compared with the data from the TDFRS experiment. The TDFRS method works with fairly small temperature differences in the order of micro to milli Kelvin and is one of the most effective methods existing today for investigation of the thermal diffusion behavior of different binary mixtures. This method avoids convection and provides accurate and reliable Soret and thermal diffusion coefficients for different kinds of liquid mixtures such as low molecular weight mixtures but also polymer solutions and colloidal suspensions [116].

\subsection{Computational details}

Reverse nonequilibrium molecular dynamics method has been applied to investigate the thermal diffusion of alkane/benzene mixtures. A detailed description can be found elsewhere [189]. The intermolecular force field contained constraints, angle bending, torsional potentials and harmonic dihedral potential. Lorentz-Berthelot mixing rules were employed for unlike nonbonded interactions. The force field parameters for benzene were taken from Milano et al [96]. The C-H bonds were slightly polarized in order to reproduce the benzene quadrupole moment. The same benzene model has been successfully used to simulated the thermal difusion properties of benzene/cyclohexane mixtures [189]. For alkanes we used the TraPPE-UA [93] force field. All $\mathrm{CH}_{n}$ groups were treated as individual atoms without taking into account electrostatic interactions. We also tried to use the force field from Nath [103], but the obtained values for the enthalpy of vaporization were also lower than in the experiment. Chang and Sandler [27] proposed a full atom force field in order to solve this problem. We are aware of the fact that the choice of the force field is crucial for calculating the thermal diffusion properties and more sophisticated models such as full atom force fields might be feasible in future simulations.

All systems were simulated at $T=303 \mathrm{~K}$ and $P=1$ atm. The YASP package [101] was 
used. The cutoff length for nonbonded interactions was $1.1 \mathrm{~nm}$. The time step was $2 \mathrm{fs}$. First, the studied mixture was equilibrated in the cubic simulation box. Then, the cell was replicated in $z$-direction $\left(\mathrm{L}_{x}=\mathrm{L}_{y}=\mathrm{L}_{z} / 3 \approx 4-4.4 \mathrm{~nm}\right)$. The diffusion coefficients were obtained from equilibrium molecular dynamics simulations (EMD) with a runtime of $350 \mathrm{ps}$. The self diffusion coefficient $D^{S}$ was calculated from the mean square displacements $\triangle r^{2}$ of the center of mass of the molecules via the Einstein relation in pure liquids [189]

$$
D^{S}=\frac{1}{6} \frac{d}{d t}\left\langle\triangle r^{2}\right\rangle
$$

The same expression has also been used the calculated the self diffusion in binary mixtures, which is often denoted as tracer diffusion. The mutual diffusion coefficient $D_{12}$ was calculated from the mean-squared displacement of the center of mass of all molecules of one species [189]

$$
D_{12}^{i}=x_{1} x_{2}\left(\frac{1}{x_{1} M_{1}}+\frac{1}{x_{2} M_{2}}\right)^{2}\left(x_{i} M_{i}\right)^{2} \frac{N\left\langle\left(\triangle r_{i}^{c m}\right)^{2}\right\rangle}{6 t}
$$

where $D_{12}^{i}$ is the diffusion coefficient, calculated from the center of mass of all molecules of the species $i, x_{i}$ and $M_{i}$ the corresponding mole fraction and molecular weight, respectively and $N$ is the total number of molecules. $D_{12}^{1}$ is equal to $D_{12}^{2}$ as far as the momentum is conserved. In Eq. 5.1 the mutual diffusion coefficient is denoted simplified as $D$. The error bars were calculated from the standard deviation among the $x, y$ and $z$ components of the diffusion coefficient. The heat of vaporisation was calculated from the intermolecular nonbonded energy $H_{\text {vap }}=-\left\langle E^{\text {inter }}\right\rangle+R T$.

All reverse nonequilibrium molecular-dynamics (RNEMD) simulations were performed at constant NVT conditions with 960 or 1500 molecules in the simulation box. The periodic system was divided into 20 slabs along the $z$ - direction. The average temperature $300 \mathrm{~K}$ was kept constant by a thermostat of Berendsen et al. [16], with the temperature coupling time being $\tau=50$ ps. The temperature gradient was created by exchanging every $\mathrm{N}_{\text {exch }}$ steps the center of mass velocity vector of two molecules ("coldest" molecule in the hot slab one and the "hottest" molecule in the cold slab 11) of the same kind. After the concentration gradient is induced the Soret coefficient can be calculated (c.f. Eq. 5.2). For each simulation run two values of the Soret coefficient were calculated: from 9 slabs of the downward branch and from 9 slabs in the upward branch. The hottest and coldest slabs have been excluded 
from the analysis. The final value of $S_{\mathrm{T}}$ represents the average value, the error bars reflect the difference between $S_{\mathrm{T}}$ from downward and upward branches.

\subsection{Equilibrium molecular dynamics simulations}

Fig. 5.1A shows a good agreement between the simulated and experimental [82] densities for $n$-heptane/benzene mixture at different concentrations. Typically, the agreement is better than $1.5 \%$. The self diffusion coefficients of benzene and $n$-heptane are $30 \%$ lower and $20 \%$ larger, respectively than the experimental value $[96,49]$. The mutual diffusion coefficient for a $n$-heptane mole fraction of 0.25 is in satisfactory agreement with the experiment, while the other two values ( $n$-heptane mole fraction of 0.5 and 0.75 ) are systematically $\approx 40 \%$ larger than in the experiment. Nevertheless, the experimental trend is reproduced.

Table 5.1 shows the experimental and the simulation density and heat of vaporization for pure benzene, $n$-heptane, 3-methylhexane (3-MH), 2,3-dimethylpentane (2,3-DMP) and 2,4dimethylpentane (2,4-DMP). The simulated densities are in satisfactory agreement with the experimental values. The branching effect has almost no influence on the heptane density. The vaporization enthalpies from simulations for benzene and heptane are also in satisfactory agreement with experiment, while their values become systematically smaller than in experiment with increasing degree of branching. Unfortunately, we did not find the values of the self diffusion coefficient of $n$-heptane and its isomers in the literature. The simulated self diffusion coefficient decreases with increasing degree of branching $(4.3,4.15,3.58$ and 3.78 $\mathrm{cm}^{2} \mathrm{~s}^{-1}$ for $n$-heptane, 3-MH, 2,3-DMP and 2,4-DMP, respectively).

Fig. 5.2A and B shows the density and diffusion coefficients for equimolar mixtures of $n$-heptane and its isomers in benzene. The densities of the branched heptane/benzene mixtures are roughly $2 \%$ larger than for the equimolar heptane/benzene mixture. The calculated mutual diffusion coefficient for branched heptane/benzene mixtures agrees better with the experimental values than for the equimolar mixture of heptane in benzene. More ideal packing of the heptane isomers in benzene can be responsible for the smaller error bars of the mutual diffusion coefficient. 


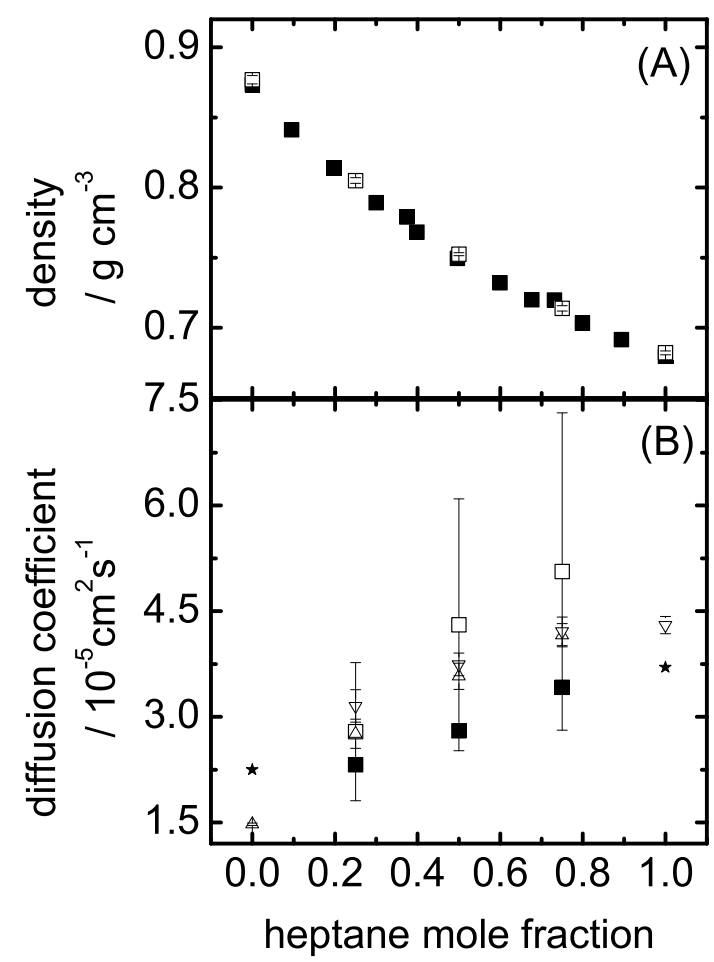

Figure 5.1: (A) Comparison between simulated ( $\square$ ) and experimental ( $\square$ ) [82] densities for $n$-heptane/benzene mixtures. (B) Comparison between simulated ( $\square$ ) and experimental ( (c.f. Chapter 4) mutual diffusion coefficients for $n$-heptane/benzene mixtures. Tracer and self diffusion coefficient of benzene $(\Delta)$ and $n$-heptane $(\nabla)$ in the mixture and in the pure liquid, respectively. Stars $(\star)$ represent the literature data for self diffusion coefficient $D^{S}$ of benzene [96] and $n$-heptane [49]. 


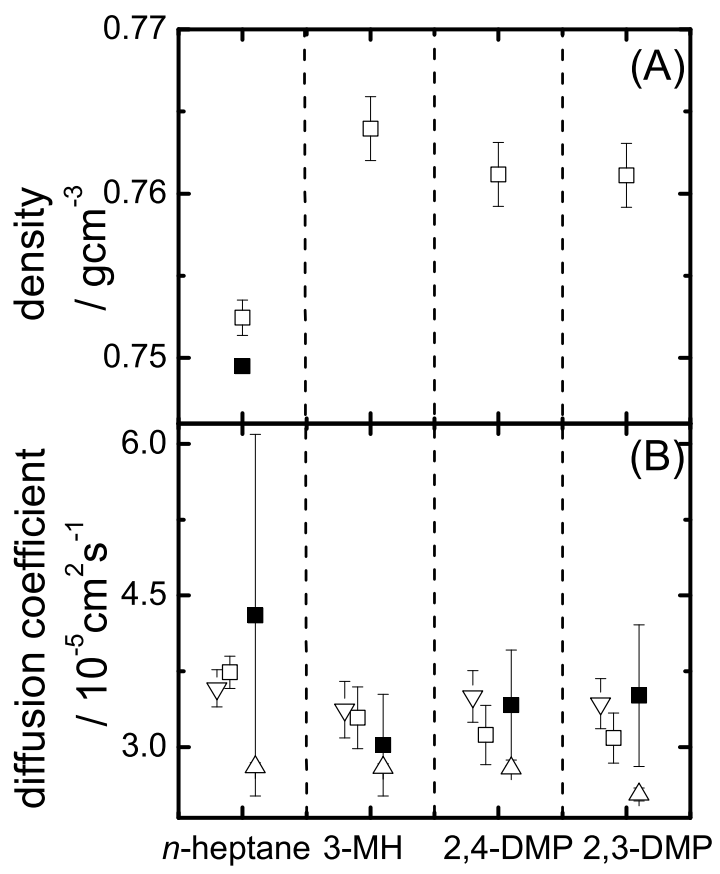

Figure 5.2: (A) The simulated ( $\square$ ) and experimental ( $\square$ ) alkane densities for equimolar mixtures of $n$-heptane and its isomers in benzene. (B) Comparison between simulated and experimental (c.f. Chapter 4) mutual diffusion coefficients $(\square, \square)$ for the same mixtures and the tracer diffusion coefficients of benzene $(\Delta)$ and of heptane and its isomers $(\nabla)$. Solid symbols refer to experimental values and open symbols to simulation results. For clarity the diffusion coefficient have been shifted by a small amount in the x-direction. 
Table 5.1: The density and the heat of vaporization of the investigated solvents from the experiment $[1,186]$, and simulations.

\begin{tabular}{|l|r|r|r|r|}
\hline substance & $\begin{array}{r}\rho_{\text {exp }} / \\
\mathrm{gcm}^{-3}\end{array}$ & $\begin{array}{r}\rho_{\text {sim }} / \\
\mathrm{gcm}^{-3}\end{array}$ & $\begin{array}{r}H_{\text {exp }}^{\text {vap }} \\
\mathrm{kJ} \mathrm{mol}^{-1}\end{array}$ & $\begin{array}{r}H_{\text {sim }}^{\text {vap } /} \\
\mathrm{kJ} \mathrm{mol}^{-1}\end{array}$ \\
\hline -heptane & 0.684 & 0.682 & 36.2 & 33 \\
3-MH & 0.687 & 0.686 & 35.2 & 27.8 \\
2,4-DMP & 0.673 & 0.681 & 33.1 & 23.6 \\
2,3-DMP & 0.695 & 0.690 & 34.1 & 21.9 \\
benzene & 0.874 & 0.877 & 34.2 & 32.5 \\
\hline
\end{tabular}

\subsection{Non equilibrium molecular dynamics simulations}

Fig. 5.3 shows the temperature and mole fraction profiles for three $n$-heptane/benzene mixtures with a molar fraction of $x_{\text {heptane }}=0.25,0.5$ and 0.75 . The average fluctuation of the concentration is $8 \%$ and temperature variation is $2 \mathrm{~K}$ in each slab. The simulations for $n$ heptane/benzene mixture with $x_{\text {heptane }}=0.25$ and 0.5 were performed with $N_{\text {exch }}=100$ and 1500 molecules in the simulation box. The simulation time was 11 and $13.7 \mathrm{~ns}$, respectively. For $x_{\text {heptane }}=0.75$ the exchange period was 250 and the number of molecules in simulation box was equal to 960 with a simulation time was 27 ns. For this concentration it was difficult to get a linear concentration gradient at lower exchange number 220 and 125 . Fig. 5.4 shows the calculated Soret coefficient in comparison with experimental data (c.f. Chapter 4). The RNEMD reproduces the experimental trend very well, however the simulated values are systematically $\approx 3 \times 10^{-3} \mathrm{~K}^{-1}$ lower than in the experiment. The same trend was observed by Zhang et al [189] for benzene/cyclohexane mixture, they found that the simulated Soret coefficient was $4 \times 10^{-3} \mathrm{~K}^{-1}$ lower than in experiment. We also investigated the influence of the exchange number on the Soret coefficient for the $n$-heptane mole fraction of 0.25 . Increasing the exchange number from 100 to 200 makes the temperature gradient roughly two times smaller. For $N_{\text {exch }}=200, x_{\text {heptane }}=0.25$ and the same simulation time we have found $S_{\mathrm{T}}=-(1.25 \pm 0.15) \times 10^{-2} \mathrm{~K}^{-1}$ which is roughly $29 \%$ lower than $S_{\mathrm{T}}=-(9.38 \pm 0.88) \times 10^{-3} \mathrm{~K}^{-1}$ obtained for $N_{\text {exch }}=100$. For the benzene/cyclohexane mixture with a molar fraction $x_{\text {benzene }}=0.25 S_{\mathrm{T}}$ for $N_{\text {exch }}=200$ had also been found to be 


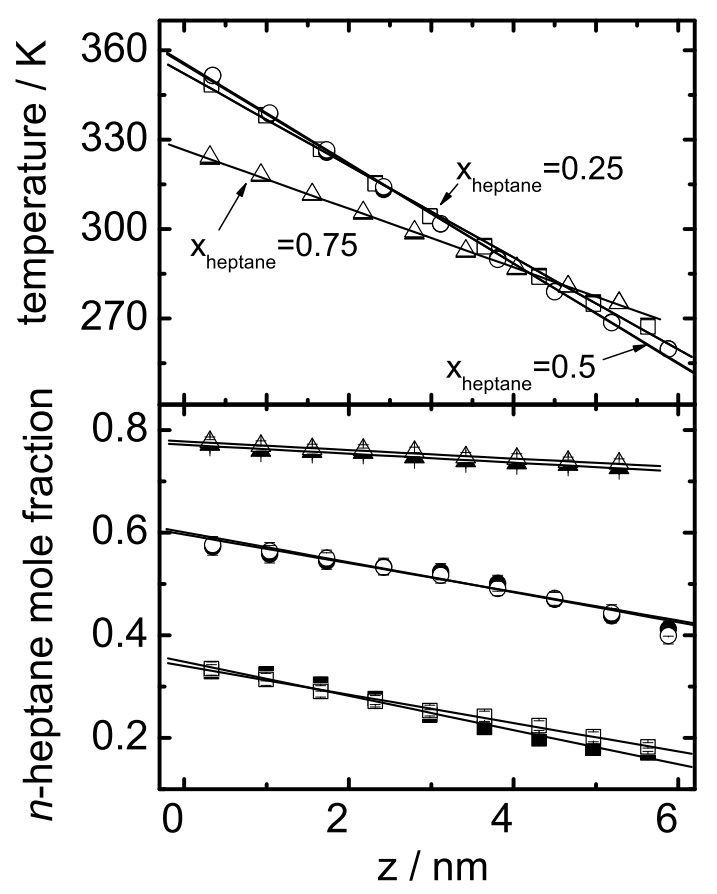

Figure 5.3: The temperature and mole fraction profiles for $n$-heptane/benzene mixtures with $x_{n \text {-heptane }}=0.25(\square, \mathbf{\square}), 0.5(\circ, \bullet)$ and $0.75(\triangle, \mathbf{\Delta})$. The solid and open symbols refer to 9 slabs of the downward and upward branch in the simulation box. The error bars in the temperature profile are smaller than the symbols, which is also reflected by the overlapping points for the upward and downward branch. The maximum error bar in the concentration profile does not exceed two symbols sizes. 


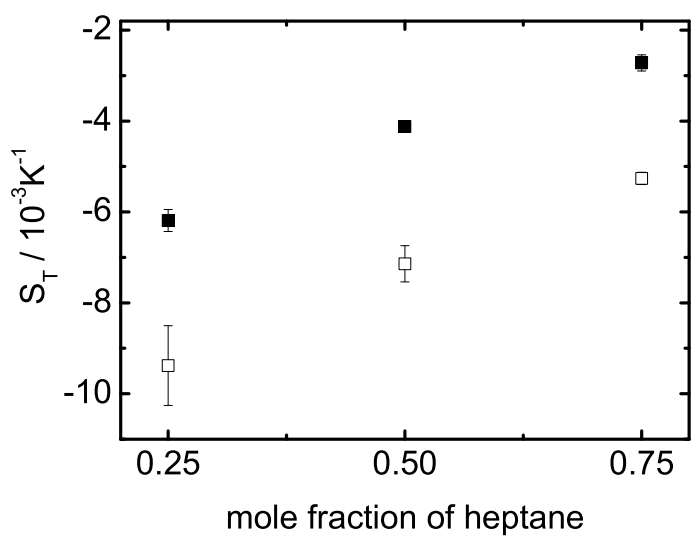

Figure 5.4: Comparison of the simulated Soret coefficients ( $\square$ ) of $n$-heptane in benzene with the experimental values (ם) (c.f. Chapter 4).

$46 \%$ lower than for $N_{\text {exch }}=100$ (Ref. 189).

We also checked the influence of the size of the rectangular simulation box $\left(L_{\mathrm{x}}=L_{\mathrm{y}}=\right.$ $\left.L_{\mathrm{z}} / 3\right)$ on the Soret coefficient for equimolar $n$-heptane/benzene mixture. The obtained value for 960 molecules in the simulation box and $N_{\text {exch }}=100$ is $(7.14 \pm 0.4) \times 10^{-3} \mathrm{~K}^{-1}$ after $10.5 \mathrm{~ns}$. This result is in perfect agreement with $S_{\mathrm{T}}=(7.16 \pm 0.22) \times 10^{-3} \mathrm{~K}^{-1}$ (c.f. Fig. 5.4) obtained for 1500 molecules in simulation box and the same exchange number averaged over 13.7ns. Therefore, we can conclude that our simulation system is large enough.

All simulations for branched heptanes (3-MH, 2,3-MH and 2,4-DMP) in benzene were performed for 960 molecules in the simulation box and with an exchange rate of $N_{\mathrm{exch}}=100$. Fig. 5.5 shows the temperature and mole fraction profiles for mixtures of the three branched heptanes in benzene averaged over $10.5 \mathrm{~ns}$.

Fig. 5.6 shows the calculated Soret coefficient in comparison with experimental data (c.f. Chapter 4). The RNEMD reproduces experimental trend very well, however the simulated values are systematically $\approx 3 \times 10^{-3} \mathrm{~K}^{-1}(\approx 25 \%)$ lower than in experiment. For 2,3-DMP/benzene system with the smallest Soret coefficient we performed a second, independent MD simulation with a simulation time of $4.5 \mathrm{~ns}$. The magnitude of the ob- 


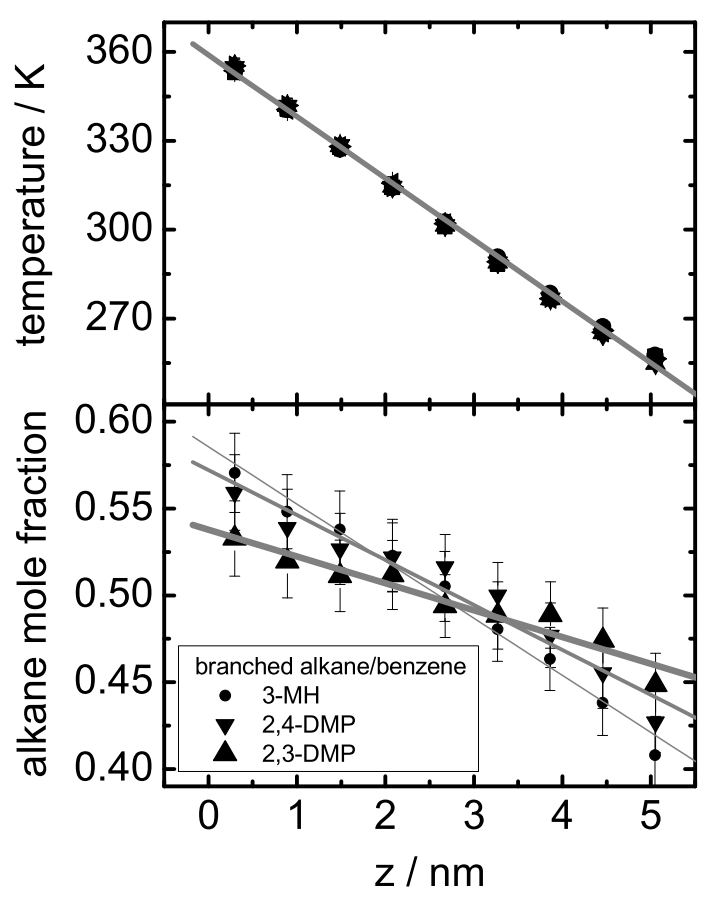

Figure 5.5: Temperature and mole fraction profiles for equimolar mixtures of 3-MH, 2,3DMP and 2,4-DMP in benzene. 
tained Soret coefficient $S_{\mathrm{T}}=3.2 \times 10^{-3} \mathrm{~K}^{-1}$ agrees within the error bars with the value $S_{\mathrm{T}}=(3.04 \pm 0.2) \times 10^{-3} \mathrm{~K}^{-1}$ obtained after $10.5 \mathrm{~ns}$.

\subsection{Discussion}

In both experiment and simulation we found for equimolar mixtures of heptane isomers and benzene that the magnitude of Soret coefficient decreases with increasing degree of branching. This result can be analyzed in terms of the work of Reith and Müller-Plathe [126]. Generally, there is no Soret effect in the mixture of absolutely equal components due to the principle of symmetry. The Soret effect is basically the response of the system to the difference between two mixing partners. In their work, they considered binary equimolar mixtures of Lennard Jones particles. The influence of the difference in mass, $m$, diameter, $\sigma$, and depth of the interaction potential, $\varepsilon$ on the Soret coefficient was investigated. They varied systematically the ratio of one of the parameters (e.g. $\mathrm{m}_{1} / \mathrm{m}_{2}$ ) while keeping the two other parameters fixed and equal. By this procedure the obtained three additive contributions $S_{\mathrm{T}}^{m}, S_{\mathrm{T}}^{\sigma}$ and $S_{\mathrm{T}}^{\varepsilon}$ of the total Soret coefficient $S_{\mathrm{T}}^{L J}$ stemming from the difference in mass, diameter and interaction strength, respectively

$$
S_{\mathrm{T}}^{L J}=S_{\mathrm{T}}^{m}+S_{\mathrm{T}}^{\sigma}+S_{\mathrm{T}}^{\varepsilon}
$$

Results indicate that heavier species, smaller species, and species with higher interaction strengths tend to accumulate in the cold region The following empirical laws were obtained by low order fits of the independent parameter variations

$$
\begin{aligned}
& S_{\mathrm{T}}^{m}=-0.7\left(\frac{m_{1}}{m_{2}}\right)^{2}+9.5\left(\frac{m_{1}}{m_{2}}\right)-8.8 \quad \text { for } \frac{m_{1}}{m_{2}} \leq 8 \\
& S_{\mathrm{T}}^{\sigma}=67.4\left(\frac{\sigma_{1}}{\sigma_{2}}\right)^{2}-179.3\left(\frac{\sigma_{1}}{\sigma_{2}}\right)+111.9 \text { for } \frac{\sigma_{1}}{\sigma_{2}} \leq 1.25 \\
& S_{\mathrm{T}}^{\varepsilon}=4.4\left(\frac{\varepsilon_{1}}{\varepsilon_{2}}\right)^{2}+3.5\left(\frac{\varepsilon_{1}}{\varepsilon_{2}}\right)-7.9 \quad \text { for } \frac{\varepsilon_{1}}{\varepsilon_{2}} \leq 1.75
\end{aligned}
$$

Table 5.2 shows the mass, the size and the interaction contribution to the Soret coefficient. The sum of these three values $\mathrm{S}_{T}^{L J}$ is compared with $S_{\mathrm{T}}$ from simulations. The mass ratio of heptanes to benzene $\left(\mathrm{m}_{1} / \mathrm{m}_{2}=1.28\right)$ is obviously not sensitive to the degree of branching, 


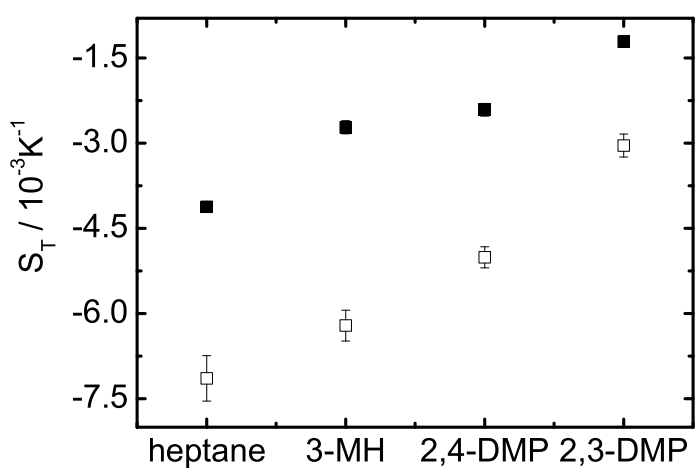

Figure 5.6: Comparison of the simulated Soret coefficients ( $\square$ ) with the experimental values (ם) (c.f. Chapter 4) for equimolar mixtures of $n$-heptane and its isomers in benzene.

so that $S_{\mathrm{T}}^{m}>0$ is the same for all four mixtures. The size contribution $\mathrm{S}_{\mathrm{T}}^{\sigma}$ can also be considered the same as far as the density of the pure branched heptanes agree within $2 \%$ with the density of pure $n$-heptane (c.f. Tab. 5.1). Quantitatively, this contribution can be estimated from the Van-der-Waals volumes $V^{V d W}$ of two mixing partners, considering $\sigma_{1} \sigma_{2}^{-1}$ $=\left(\mathrm{V}_{1}^{V d W} / \mathrm{V}_{2}^{V d W}\right)^{1 / 3}$. The Van-der-Waals volume of a molecule can be determined by atomic increments [47]. The Van-der-Waals volume of benzene $48.4 \mathrm{~cm}^{3} \mathrm{~mol}^{-1}$ is less than the Van-der-Waals volume of $n$-heptane, 3-MH, 2,3-DMP, 2,4-DMP (78.49, 78.48, 78.47, 78.47 $\mathrm{cm}^{3} \mathrm{~mol}^{-1}$ ) (Ref. [186]). It turns out, that the size contribution $S_{\mathrm{T}}^{\sigma}$ is negative and not sensitive to the degree of branching. The third term in Eq. $5.5 S_{\mathrm{T}}^{\varepsilon}$ can be estimated from the vaporization enthalpies of the pure components, considering $\varepsilon_{1} / \varepsilon_{2}=\mathrm{H}_{1}^{v a p} / \mathrm{H}_{2}^{v a p}$ (c.f. Chapter 2). For calculation of $S_{\mathrm{T}}^{\varepsilon}$ we used the vaporization enthalpies from the simulations (c.f. Tab. 5.1). Thus, the (constant) size contribution $S_{\mathrm{T}}^{\sigma}<0$ dominates the (constant) mass contribution $S_{\mathrm{T}}^{m}>0$ making $S_{\mathrm{T}}$ negative. $\mathrm{S}_{T}^{L J}$ calculated in this way varies only because of the interaction strength differences and it was found to be a decreasing function of degree of branching, what contradicts to the trend, observed by simulation and experimentally. It is therefore clear that a simple analysis which maps the complex molecules onto simple Lennard-Jones particles is not sufficient. This is consistent with earlier results on benzene-alkane mixtures [189]. The 
Table 5.2: The mass, the size and the interaction contributions to the Soret coefficient $\left(10^{-3}\right.$ $\mathrm{K}^{-1}$ ) calculated using Eq. 5.6, 5.7 and 5.8. The sum of these three contributions $\mathrm{S}_{T}^{L J}$ is compared with simulation results $\mathrm{S}_{T}^{\text {sim }}$.

\begin{tabular}{|l|r|r|r|r|c|}
\hline mixture & $S_{T}^{m}$ & $S_{T}^{\sigma}$ & $S_{T}^{\varepsilon}$ & $S_{T}^{L J}$ & $S_{T}^{\text {sim }}$ \\
\hline n-heptane/benzene & 2.23 & -5.72 & 0.11 & -3.38 & -7.14 \\
3-MH/benzene & 2.23 & -5.72 & -1.68 & -5.17 & -6.21 \\
2,4-DMP/benzene & 2.23 & -5.72 & -3.04 & -6.53 & -5.01 \\
2,3-DMP/benzene & 2.23 & -5.72 & -3.54 & -7.03 & -3.04 \\
\hline
\end{tabular}

different nature of the benzene-benzene and alkane-alkane interactions due to the benzene quadrupole moment could also contribute to the Soret coefficient. However, the quantitative estimation of this contribution is difficult. The branching effect, probably, can be considered by taking into account the non - ideality of alkane/benzene mixtures caused by their anisotropy in shape.

There is another way of interpreting the Soret effect in terms of simple molecular properties: the moments of inertia have been shown to make an important contribution to the Soret effect for mixtures of cyclohexane and benzene isotopes [36]. Debuschewitz and Köhler found that the Soret coefficient could be written as a sum of three contributions:

$$
S_{T}=a_{M} \delta M+b_{I} \delta I+S_{T}^{0}
$$

where $\delta M=\left(M_{1}-M_{2}\right)\left(M_{1}+M_{2}\right)^{-1}$ and $\delta I=\left(I_{1}-I_{2}\right)\left(I_{1}+I_{2}\right)^{-1}$ are the relative differences of the masses $\left(M_{1}, M_{2}\right)$ and moments of inertia $\left(I_{1}, I_{2}\right)$ of the molecules, respectively. A further investigation of the isotope effect [184] suggested that the absolute rather than the relative differences between the masses and moments of inertia should enter the expression for the Soret coefficient so that the difference terms in Eq. (5.9) are given by $\delta M=M_{1}-M_{2}$ and $\delta I=I_{1}-I_{2}$. In the Chapter 4 we have calculated the moments of inertia about the symmetry axis $\left(I_{z z}\right.$ for the disk-like benzene molecules, $I_{x x}$ for the $n$-heptane and five heptane isomers, including 3-MH, 2,3-DMP and 2,4-DMP) using an atomistic model for single molecules in vacuum [5]. It was found that the Soret coefficient correlates almost increasing linearly with the moment of inertia $I_{x x}$ in the direction of the main chain. Based on this observation one 
might conclude that the Soret coefficient is completely determined by the moment of inertia, but simultaneously with the change of the moment of inertia (kinetic contribution) also the anisometry (static contribution) of the molecule changes. The chemical contribution $S_{T}^{0}$ (c.f. Eq. 5.9) can be associated with the size (Eq. 5.7) and the interactions (Eq. 5.8) effects. Reith and Müller-Plathe [126] have shown that it is possible to combine Eq. 5.7 and 5.8 into a single one which involves the cohesive energy densities $e=\varepsilon / \sigma^{3}$. In our case this parameter is not sensitive to the mixture (c.f. Table 5.1), so that the chemical contribution $S_{T}^{0}$ (c.f. Eq. 5.9) turns out to be the same for all four equimolar heptane/benzene mixtures, if we consider experimental evaporization enthalpies $H_{\exp }^{\text {vap }}$ (c.f. Table 5.1).

The shape of the molecules influences their packing. The packing can be analyzed using the radial distribution functions (RDF). Fig. 5.7A shows the centre-of-mass RDF for pure $n$-heptane, 3-MH, 2,3-DMP, 2,4-DMP and benzene. The characteristic distance $r$ at which fluctuations in RDF disappear as well as the amplitude and the position of the first maximum become larger with increasing branching and correlate with the magnitude of the Soret coefficient (c.f. Fig. 5.6). For pure benzene the packing is even closer to the spherical packing than for alkanes. The RDF, calculated between centers of mass of benzene and alkanes in the equimolar alkane/benzene mixtures (c.f. Fig. 5.7B) shows the same trend as the center of mass RDF, calculated for pure components (c.f. Fig. 5.7A). The packing efficiency can also be analyzed in terms of the smallest distance between centers of masses of molecules, which can be estimated from the integral of RDF (c.f. Fig. 5.8). The $n$-heptane- $n$-heptane distance in the pure state and the $n$-heptane-benzene distance in the equimolar mixture are almost equal to each other and less than the benzene-benzene distance in pure state. This means that the benzene rings orient parallel to the $n$-heptane chains and do not disturb the packing of $n$-heptane significantly. In the pure state benzene molecules arrange themselves in a T-shape geometry [96] resulting in a larger distance between the centers of masses. The behavior of the branched heptanes is different due to their increasingly globular shape. The alkane-alkane distance in pure liquid and benzene-alkane distance in the mixture becomes larger with increasing branching and correlates also with the magnitude of the Soret coefficient (c.f. Fig. 5.6).

The packing effect can influence the diffusion properties of the mixtures. For heptane/benzene mixture the tracer diffusion coefficient of benzene is smaller than the tracer 


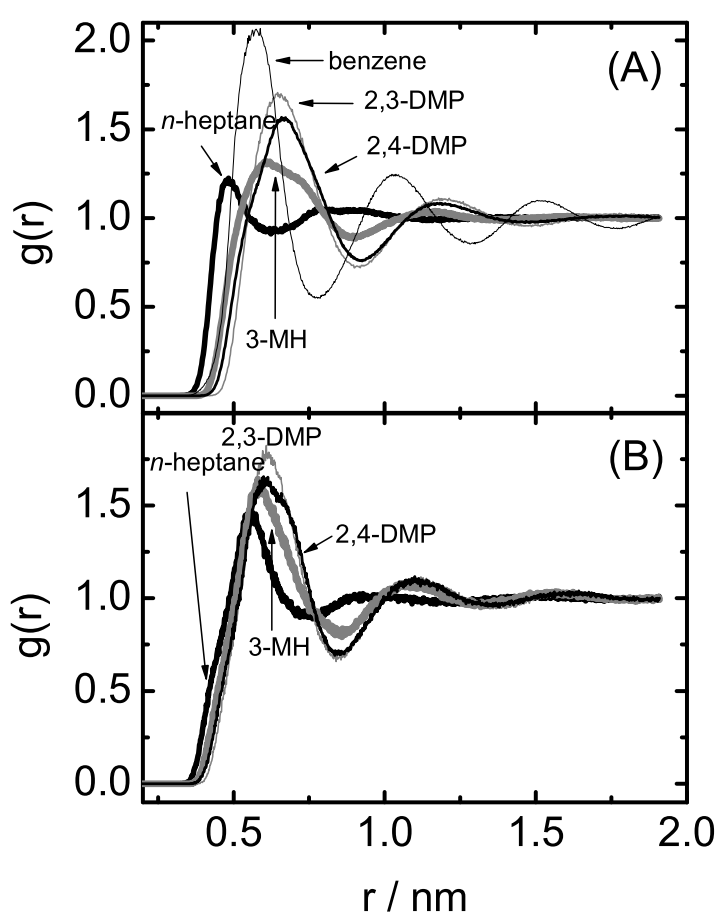

Figure 5.7: (A) Center of mass radial distribution function for pure components. (B) Center of mass alkane-benzene radial distribution function for equimolar mixtures. 


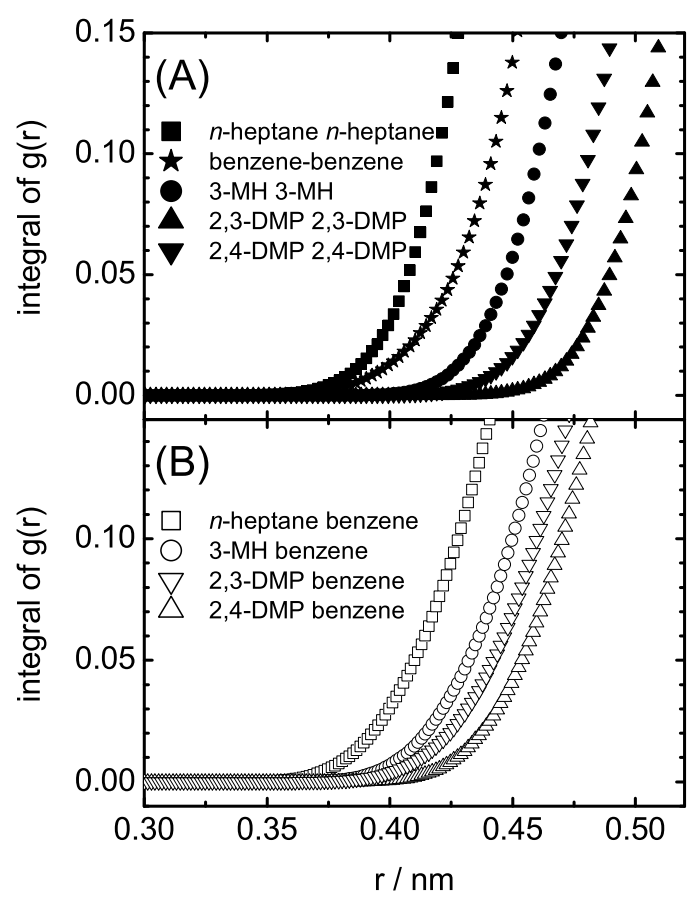

Figure 5.8: Integral of the center of mass radial distribution function for pure components and for equimolar mixture between benzene and alkanes. 
diffusion coefficient of heptane for all investigated concentrations (c.f. Fig. 5.1 B). At the same time their differences decrease with increasing concentration of heptane. This could be due to the higher packing of benzene with heptane molecules in the heptane rich region.

\subsection{Conclusion}

We applied a equilibrium molecular dynamics and the reverse nonequilibrium moleculardynamic algorithm to calculate the mutual diffusion, tracer diffusion and Soret coefficients in different alkane/benzene mixtures. In order to explore the concentration influence we studied $n$-heptane/benzene mixture at different concentrations. Additionally, we looked into the influence of the degree of branching by investigating equimolar mixtures of the branched heptanes (3-MH, 2,3-DMP, 2,4-DMP) in benzene. The simulated Soret and mutual diffusion coefficients show the same trend as in experiment. However, the simulated values of $S_{\mathrm{T}}$ values are systematically $\approx 3 \times 10^{-3} \mathrm{~K}^{-1}$ lower than in the experiment. The observed decreasing of the magnitude of $S_{\mathrm{T}}$ for equimolar alkane/benzene mixtures with branching of the alkane can not be explained by mass and size effects. Nevertheless we observe a linear increase of $S_{\mathrm{T}}$ with increasing moment of inertia, which could be purely to kinetic but also due to static contributions due to simultanous change of the anisometry of the molecules. The effect of the molecular shape, which affects the liquid structure, as well as kinetic properties of the mixture, needs to be considered additionally. We have, however, not found a simple relation to take branching or, more generally, molecular shape, into account. 


\title{
Systematic study of the thermal diffusion in associated mixtures
}

\begin{abstract}
We performed systematic temperature and concentration dependent measurements of the Soret coefficient in different associated binary mixtures of water, deuterated water, dimethyl sulfoxide (DMSO), methanol, ethanol, acetone, methanol, 1-propanol, 2-propanol, propionaldehyde using the so called thermal diffusion forced Rayleigh scattering method. For some of the associating binary mixtures such as ethanol/water, acetone/water and DMSO/water the concentration $x_{w}^{ \pm}$at which the Soret coefficient changes its sign does not depend on temperature and is equal to the concentration $x_{w}^{\times}$where the Soret coefficient isotherms intersect. While for others such as 1-propanol/water, 2-propanol/water and ethanol/DMSO the sign change concentration is temperature dependent, which is the typical behavior observed for non-associating mixtures. For systems with $x_{w}^{ \pm}=x_{w}^{\times}$we found that $x_{w}^{ \pm}$depends linearly on the ratio of the vaporization enthalpies of the pure components. Probably due to the similarity of methanol and DMSO we do not observe a sign change for this mixture. The obtained results are related to structural changes in the fluid observed by nuclear magnetic resonance, mass specrometric and $X$-ray experiments in the literature. Furthermore we discuss the influence of hydrophilic and hydrophobic interactions and the solubility on thermal diffusion behavior. *
\end{abstract}

* The work described in this chapter is based on J. Chem. Phys. 128, 034505, 2008 


\subsection{Introduction}

Thermal diffusion describes the migration of molecules in a temperature gradient. For a binary mixture in a temperature gradient $\nabla T$, the enrichment of one component $\nabla x$ is characterized by the Soret coefficient $S_{\mathrm{T}}$, as

$$
S_{\mathrm{T}}=-\frac{1}{c(1-c)} \frac{|\nabla c|}{|\nabla T|}
$$

A positive Soret coefficient of the component with the weight fraction $c$ implies that this component moves to the cold region of the fluid.

The main practical applications are separation processes [145, 29] such as thermal field flow fractionation of polymers and colloids or isotope separation, characterization of geochemical processes [66, 30] and combustion [128]. Although the discovery of the effect by Ludwig [89] dates back 150 years, even qualitative predictions for liquids, which are of practical importance, are often difficult.

Even less than 20 years ago different experimental techniques such as thermo gravitational columns, beam deflection, utilizing diffusion cell and thermal diffusion forced Rayleigh scattering (TDFRS) gave different results for the simple organic mixture of toluene/n-hexane $[46,75,86]$. Later the deviations could be resolved $[188,20]$ and additionally a benchmark test has been initiated, to measure the Soret coefficient $S_{\mathrm{T}}$ in simple organic mixtures by different experimental techniques [117]. In the recent years it was also demonstrated that the TDFRS method gives reliable results for aqueous systems and compares well with other experimental techniques $[78,188,45,74]$. But recent studies on a non-ionic surfactant by the TDFRS technique showed, that the small amount of dye, which is added to convert light into heat energy, can influence the thermal diffusion behavior of the surfactant system [110, 109]. Therefore special care needs to be taken for systems with complex phase behavior.

Conceptually, binary mixtures of simple molecules can be divided into three groups: mixture of spherical molecules without specific interactions, mixtures of non-spherical molecules without specific interactions and associated mixtures. In the first group of mixtures the component with the larger mass or higher density moves to the cold side, and this effect becomes stronger if the components are less miscible $[179,126,56]$ (c.f. Chapter 2). This empirical observations still hold for some mixtures from the second group [179] such as ben- 
zene/carbon tetrachloride [40] and cyclohexane/carbon tetrachloride [159] while for systems benzene/cyclohexane [183] and benzene/alkanes (c.f. Chapters 4 and 5) this approach fails. In order to study the influence of molecular mass more systematically binary mixtures of unpolar solvents with cyclohexane and its isotopes have been investigated [183]. It turned out that the change in $S_{\mathrm{T}}$ after isotopic substitution of cyclohexane, neither depends on concentration nor on the nature of the mixing partner. Only in the case of polar acetone $\Delta S_{\mathrm{T}}$ is approximately $30 \%$ larger but still concentration independent. This investigation has also been extended to a broader temperature range [185].

Associated mixtures often show a sign change of the Soret coefficient with concentration $[111,69,92]$ so that the direction of the thermal diffusion process is predominantly guided by excess properties and not by the properties of the mixing partners like the difference in mass or moment of inertia. Such behavior is expected from the non-ideality of such mixtures due to the hydrogen bond formation. On the other hand it was observed that the sign change concentration correlates with the concentration at which the hydrogen bond network breaks down [109] and the concentration dependence of the Soret coefficient in aqueous systems seems to be universal [180]. Therefore it might be possible to relate the sign change concentration with properties of the pure components and the structure of the mixture.

In this chapter we study the thermal diffusion behavior of different binary mixtures of polar molecules like water, dimethyl sulfoxide (DMSO), ethanol, acetone, methanol, 1-propanol, 2-propanol, propionaldehyde at different temperatures and concentrations. In order to investigate the mass effect normal water $\mathrm{H}_{2} \mathrm{O}$ was replaced by $\mathrm{D}_{2} \mathrm{O}$. Particular attention has been given to the sign change concentrations. We also looked at the influence of dye (basantol yellow) on the Soret coefficient of ethanol/water mixtures under typical conditions of the TDFRS experiments.

\subsection{Experiment}

\subsubsection{Sample Preparation.}

Methanol (99.8\%), 1-propanol (99.9\%), 2-propanol (99.9\%), DMSO (99.7\%), propionaldehyde $(97 \%)$, deuterated water $(99.96$ atom \% D) were purchased from Sigma-Aldrich and ethanol (99.5\%) was ordered from Laborchemie Handels-GmbH. We took deionized Milli-Q 
water. All chemicals were used without further purification. The mixtures were prepared as follows: First a very small amount (roughly $10^{-6} \mathrm{wt} \%$ ) of the dye basantol yellow [110], was dissolved in the solvents. For each solution, the optical density was adjusted to 2 - 3 $\mathrm{cm}^{-1}$ at a wavelength of $488 \mathrm{~nm}$. Samples for the TDFRS measurements were prepared just before the measurement to avoid evaporation. The solutions were directly filtered into the sample cells (Spartan, $0.45 \mu \mathrm{m}$ ). The temperature was controlled by a circulating water bath with a temperature stability of $\Delta T= \pm 0.01 \mathrm{~K}$.

\subsubsection{Refractive index increment measurements.}

Refractive index increments with concentration $(\partial n / \partial c)_{p, T}$ at a constant pressure and temperature were measured using an Abbe refractometer. The temperature derivatives of the refractive index $(\partial n / \partial T)_{p, c}$ at a constant pressure and concentration were determined in a temperature range $T \pm 3{ }^{\circ} \mathrm{C}$ using a Michelson interferometer [14].

\subsubsection{TDFRS experiment and data analysis}

The principle of the TDFRS method is described elsewhere in details [113]. An argon-ion laser $\left(\lambda_{w}=488 \mathrm{~nm}\right)$ is used for writing the temperature grating into the sample. The laser beam is split into two writing beams of equal intensity by a beam splitter. An intensity grating is created in the sample by the interference of two laser beams. A small amount of dye in the sample converts the intensity grating into a temperature grating, which in turn causes a concentration grating by the effect of thermal diffusion. Both gratings contribute to a combined refractive index grating, which is read out by diffraction of a third laser beam $\left(\lambda_{r}=633 \mathrm{~nm}\right)$.

The intensity $\zeta_{\text {het }}(t)$ of the heterodyne signal normalized to the thermal signal is related to the Soret coefficient

$$
\zeta_{\text {het }}(t)=1-A\left(1-e^{-q^{2} D t}\right)
$$

with

$$
A=\left(\frac{\partial n}{\partial T}\right)_{p, c}^{-1}\left(\frac{\partial n}{\partial c}\right)_{p, T} c(1-c) S_{\mathrm{T}}
$$


where $q$ is the grating vector, $D$ is the mutual diffusion coefficient and $A$ is the amplitude of the concentration signal.

To determine the transport coefficients, Eq. 6.2 is fitted to the measured heterodyne signal (c.f. Fig. 6.1a) using two contrast factors $(\partial n / \partial c)_{p, T}$ and $(\partial n / \partial T)_{p, c}$ which are measured separately. The fit residuals are generally less than $1 \%$, even for the mixture with the highest dye content. However a small systematic trend can be observed, which is due to the dye contribution as a third component to the concentration signal. Those systematic deviations vanish, if one accounts for dye contribution by a two mode analysis [110, 109](c.f. Eq. 6.3).

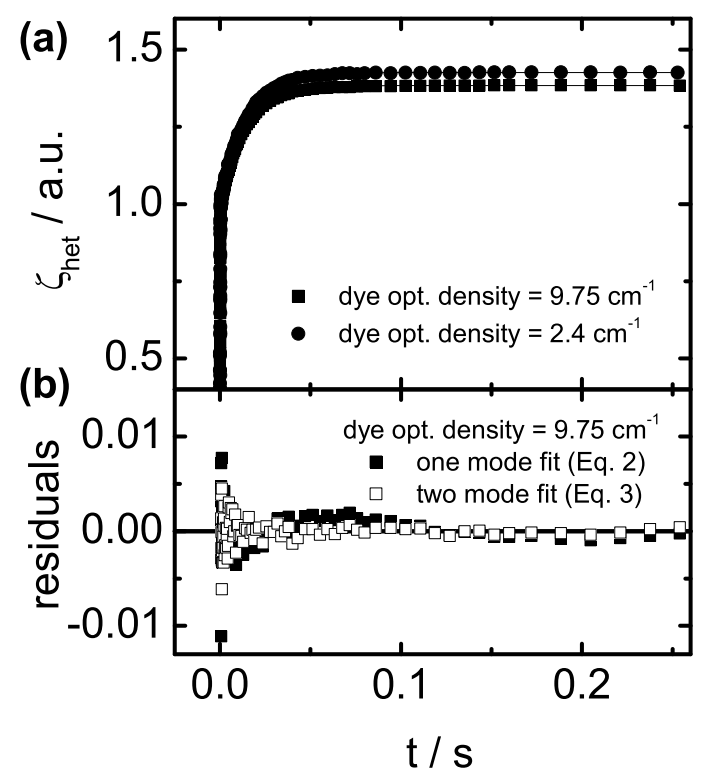

Figure 6.1: (a) Typical normalized TDFRS signals for an ethanol/ $\mathrm{D}_{2} \mathrm{O}$ mixture $\left(x_{w}=0.954\right)$ at different dye optical densities $\mathrm{OD}=9.8 \mathrm{~cm}^{-1}(\boldsymbol{\square})$ and $\mathrm{OD}=2.4 \mathrm{~cm}^{-1}(l)$, respectively. (b) The residual plot according to the one mode (Eq. 6.2, $\mathbf{\square}$ ) and the two mode analysis [109] (Eq. 6.3, $\square$ ) for ethanol/ $\mathrm{D}_{2} \mathrm{O}$ at a high dye content $\left(\mathrm{OD}=9.8 \mathrm{~cm}^{-1}\right)$. 


$$
\begin{aligned}
\zeta_{\text {het }}(t)=1 & -A_{\text {fast }}\left(1-e^{-q^{2} D_{\text {fast }} t}\right) \\
& -A_{\text {slow }}\left(1-e^{-q^{2} D_{\text {slow }} t}\right)
\end{aligned}
$$

Now we want to study in more detail the influence of the added dye and the influence of the laser intensity. Ideally the dye is inert, which means that there is no photo bleaching and no dye contribution to the diffraction signal. In order to study the dye influence, we varied the optical density between $2 \mathrm{~cm}^{-1}-10 \mathrm{~cm}^{-1}$ and investigated the thermal diffusion behavior of ethanol/ $/ \mathrm{D}_{2} \mathrm{O}$ and ethanol $/ \mathrm{H}_{2} \mathrm{O}$ mixtures with a water mole fraction of 0.697 and 0.954 .

Fig. 6.1 a shows the heterodyne signal for ethanol/ $\mathrm{D}_{2} \mathrm{O}$ for two different optical densities. The dependences of both amplitude and diffusion coefficient versus dye optical density for ethanol/ $\mathrm{D}_{2} \mathrm{O}$ (molar fraction of water $x_{w}=0.954$ ) are presented in Fig. 6.2, respectively. The contribution of the dye as a third component becomes weaker with decreasing dye content and disappears at low optical densities to the extend that a two mode analysis is no longer possible.

The increasing amplitude with dye content could be either due to screened electrostatic interactions by the charged dye molecules or due to convection problems, because the increasing dye optical density leads also to a larger temperature gradient. In order to investigate the role of convection we performed also laser intensity dependent measurements at constant dye content (c.f. Fig. 6.3). Extrapolation of both amplitude and diffusion coefficient to zero dye optical content (c.f. Fig. 6.2, $A_{\mathrm{OD} \rightarrow 0}=0.431, D_{\mathrm{OD} \rightarrow 0}=7.07 \times 10^{-6} \mathrm{~cm}^{2} \mathrm{~s}^{-1}$ ) and to zero laser intensity (c.f. Fig. 6.3, $A_{\mathrm{OD} \rightarrow 0}=0.429, D_{\mathrm{OD} \rightarrow 0}=7.14 \times 10^{-6} \mathrm{~cm}^{2} \mathrm{~s}^{-1}$ ) agree within $0.5 \%$ and $1 \%$, respectively. Therefore it is sufficient for the aqueous systems to perform intensity dependent measurements at a low optical density around 2.5 and extrapolate to zero laser intensity. At the same time, the quantitative estimation of each contribution, convection and screening electrostatic interactions by dye molecules is difficult.

\subsection{Results}

We performed systematic measurements of the Soret coefficient in different associated mixtures at different temperatures as a function of concentration. Fig. 6.4 shows the Soret coefficient for ethanol/water mixtures at different temperatures and concentrations. Figure 6.4a 


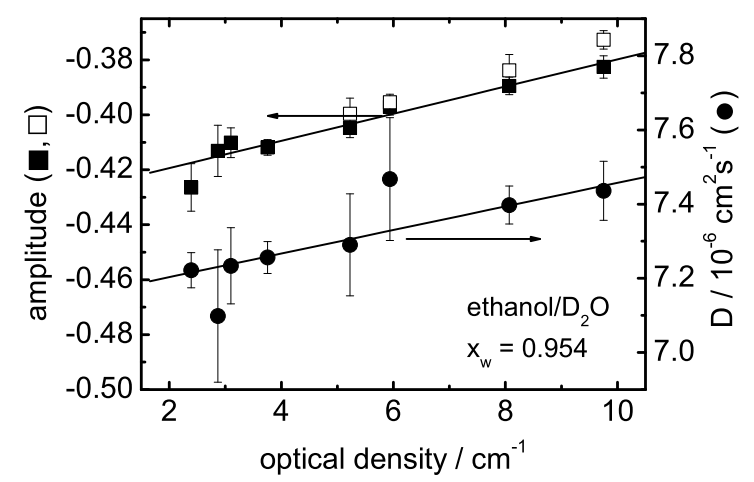

Figure 6.2: Careful check of the influence of the dye on the signal. Amplitude and diffusion coefficient $D$ of ethanol/ $/ \mathrm{D}_{2} \mathrm{O}$ mixtures $\left(x_{w}=0.954\right)$ as a function of the dye optical density. Solid $(\square, \bullet)$ and open $(\square)$ data points are obtained from the one (c.f. Eq. 6.2) and two mode (c.f. Eq. 6.3) analysis, respectively. With a linear fit (solid line) the corresponding amplitude and diffusion coefficient can be determined at $\mathrm{OD}=0$.

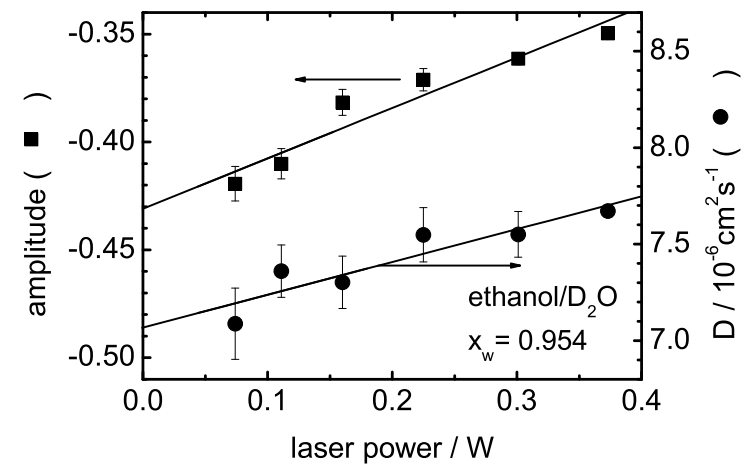

Figure 6.3: Amplitude (ם) and diffusion coefficient $D(\bullet)$ of ethanol/ $/ \mathrm{D}_{2} \mathrm{O}$ mixtures at a molar fraction of water $x_{w}=0.954$ as function of the laser intensity in front of the cell at $\mathrm{OD}=3.7 \mathrm{~cm}^{-1}$. 
shows the influence of the temperature and Fig. 6.4b the effect of the isotopic substitution of $\mathrm{H}_{2} \mathrm{O}$ by $\mathrm{D}_{2} \mathrm{O}$. A positive Soret coefficient indicates that the ethanol molecules tend to move to the cold side. There are two characteristic points in Fig. 6.4a: the molar fraction of the solvent $x_{s}$ at which $S_{\mathrm{T}}$ changes its sign $x_{s}^{ \pm}=0.142$ and the concentration at which $S_{\mathrm{T}}$ is not sensitive to the temperature $x_{s}^{\times}=0.142$ in the investigated temperature range between $T=25^{\circ} \mathrm{C}$ and $40^{\circ} \mathrm{C}$. In contrast to non-associated mixtures [185] both concentrations are the same for this aqueous mixture. The isotopic shift of $\Delta S_{\mathrm{T}}=0.85 \times 10^{-3} \mathrm{~K}^{-1}$ (Fig. 6.4b) does not depend on the concentration which is in agreement with the results for non-associated mixtures [183].

The data for DMSO/water are presented in Fig. 6.5 in the same way as for ethanol/water. Fig. 6.5a shows the temperature influence and Fig. 6.5b the effect of isotopic substitution. A positive Soret coefficient for DMSO in water implies that DMSO molecules accumulate at the cold side. The sign change concentration is also for this mixture not sensitive to the temperature $\left(x_{s}^{\times}=x_{s}^{ \pm}=0.195\right)$ and the isotopic shift of $\Delta S_{\mathrm{T}}=0.42 \times 10^{-3} \mathrm{~K}^{-1}$ is independent of concentration. Compared to the system ethanol/water the sign change concentration $x_{s}^{ \pm}=$ 0.219 for DMSO/water is higher. On the other hand the isotopic shift $\Delta S_{\mathrm{T}}$ is larger for the system ethanol/water compared to DMSO/water.

The Soret coefficient for 1-propanol/water at different temperatures and concentrations is plotted in Fig. 6.6. Also 1-propanol moves at low solvent content to the cold side, which is indicated by a positive Soret coefficient. In contrast to the systems ethanol/water and $\mathrm{DMSO} /$ water the sign change concentration depends on temperature and is equal to $x_{s}^{ \pm}=$ 0.078 and 0.108 for 25 and $40^{\circ} \mathrm{C}$, respectively. For the investigated temperatures the intersection concentration of $x_{s}^{\times}=0.064$ is lower than $x_{s}^{ \pm}$.

Fig. 6.7 shows the Soret coefficient for methanol/DMSO and ethanol/DMSO at different concentrations and temperatures. The system methanol/DMSO does not show a sign change, while for the system ethanol/DMSO a sign change occurs at about $x_{\text {DMSO }}^{ \pm}=0.4$ and 0.45 at 25 and $40^{\circ} \mathrm{C}$, respectively. For this system the sign change concentration varies with temperature. With increasing temperature the magnitude of $S_{\mathrm{T}}$ for methanol/DMSO mixture decreases for all concentrations by the same amount of $\Delta S_{\mathrm{T}} \simeq 0.5 \times 10^{-3} \mathrm{~K}^{-1}$. For ethanol/DMSO the temperature effect depends on the concentration and an intersection point can be observed in ethanol rich region, while the system methanol/DMSO does not have an intersection point at all. 


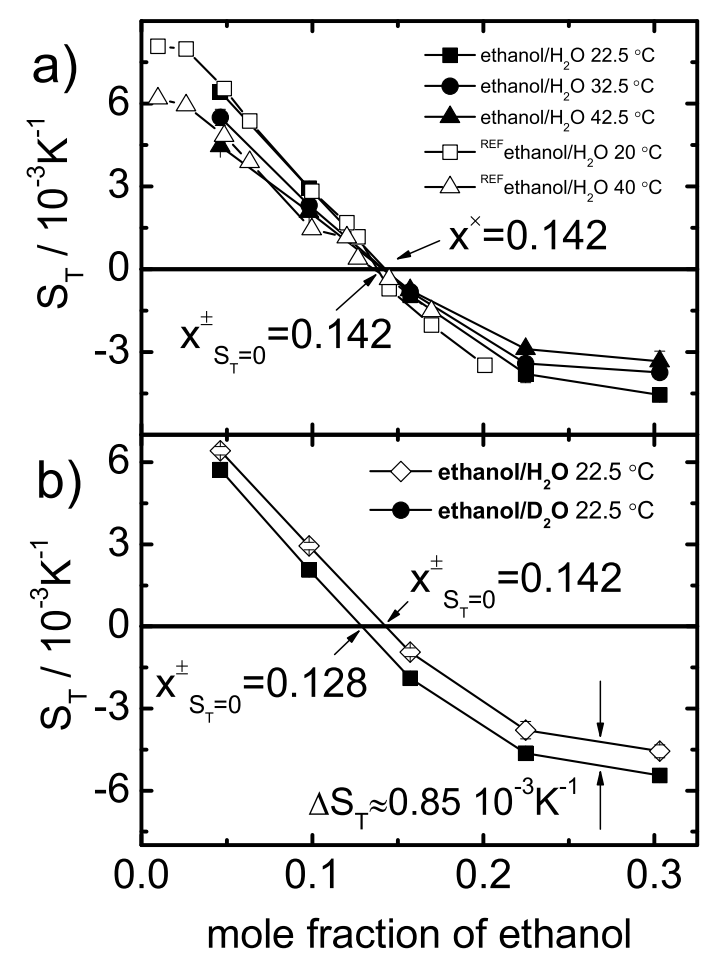

Figure 6.4: (a) Soret coefficient $S_{\mathrm{T}}$ of ethanol/ $\mathrm{H}_{2} \mathrm{O}$ mixtures at three different temperatures $22.5^{\circ} \mathrm{C}(\boldsymbol{\square}), 32.5^{\circ} \mathrm{C}(\boldsymbol{O})$ and $42.5^{\circ} \mathrm{C}(\boldsymbol{\Delta})$. The open symbols are data from Kolodner et. al [78] at $20(\square)$ and $40^{\circ} \mathrm{C}(\triangle)$. (b) Soret coefficient $S_{\mathrm{T}}$ of ethanol/ $\mathrm{H}_{2} \mathrm{O}(\diamond)$ and ethanol/ $\mathrm{D}_{2} \mathrm{O}$ (घ) mixtures at $22.5^{\circ} \mathrm{C}$. 


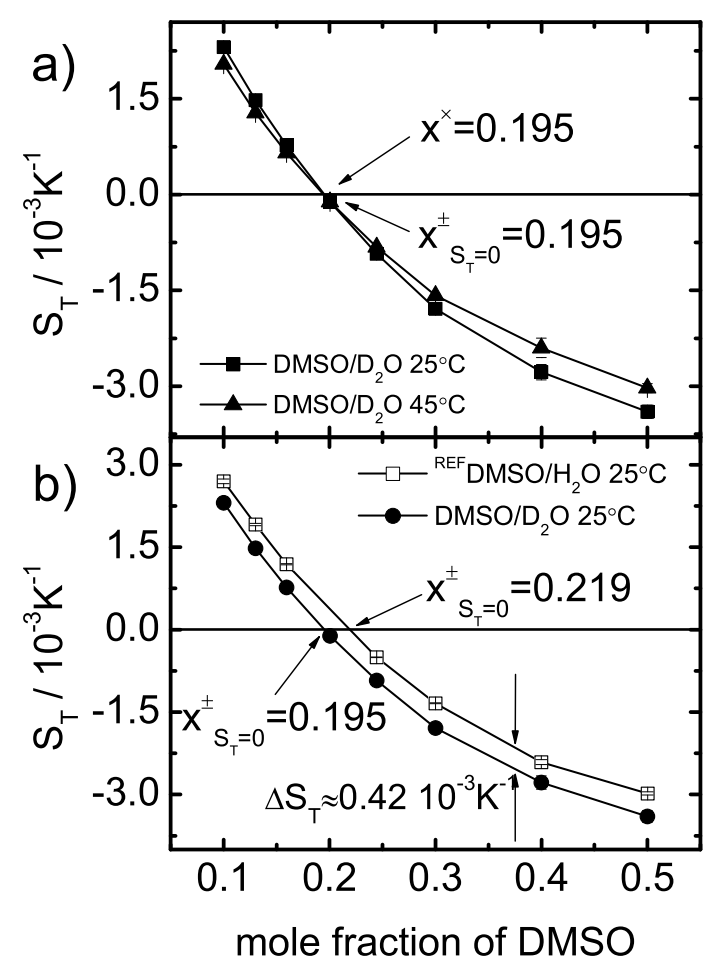

Figure 6.5: (a) Soret coefficient $S_{\mathrm{T}}$ of DMSO/ $\mathrm{D}_{2} \mathrm{O}$ mixtures at $25^{\circ} \mathrm{C}(\boldsymbol{\square})$ and $45^{\circ} \mathrm{C}(\boldsymbol{\Delta})$. (b) Soret coefficient $S_{\mathrm{T}}$ of $\mathrm{DMSO} / \mathrm{H}_{2} \mathrm{O}[111](\square)$ and $\mathrm{DMSO} / \mathrm{D}_{2} \mathrm{O}(\bullet)$ mixtures at the temperature of $25^{\circ} \mathrm{C}$. The error bars do not exceed the symbol size. 


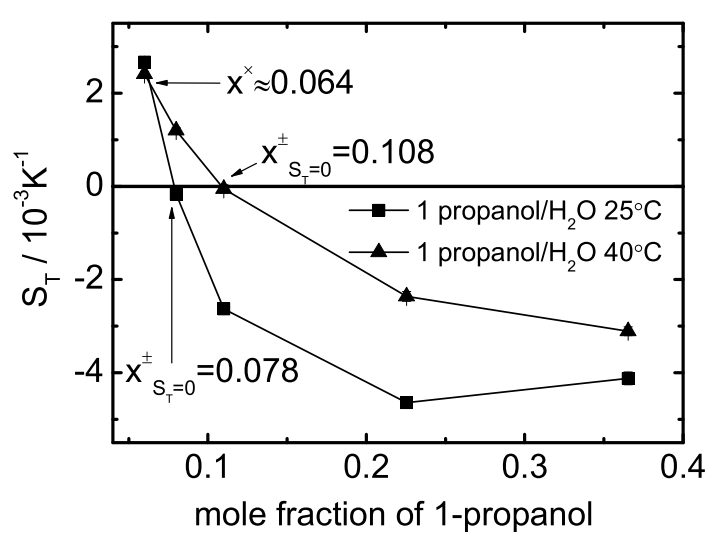

Figure 6.6: Soret coefficient $S_{\mathrm{T}}$ of 1-propanol/water mixture at $25^{\circ} \mathrm{C}(\boldsymbol{\square})$ and $40^{\circ} \mathrm{C}(\boldsymbol{\Delta})$.

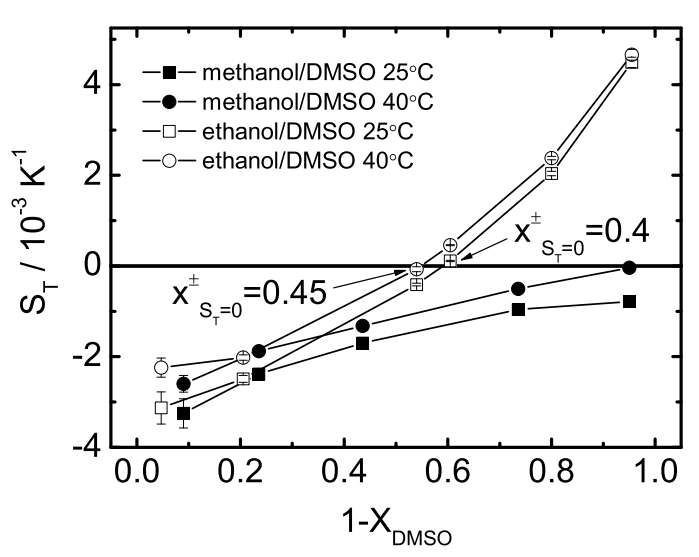

Figure 6.7: Soret coefficient $S_{\mathrm{T}}$ of ethanol/DMSO $(\square, \bigcirc)$ and methanol/DMSO $(\square, \bullet)$ mixtures at different concentrations at $25^{\circ} \mathrm{C}(\square, \boldsymbol{\square})$ and $40^{\circ} \mathrm{C}(\bigcirc, \bigcirc)$. 


\subsection{Discussion}

Figures 6.4a, 6.5a and 6.6 show the Soret coefficients for ethanol/ $\mathrm{H}_{2} \mathrm{O}$, DMSO/ $\mathrm{D}_{2} \mathrm{O}$ and 1propanol/ $/ \mathrm{H}_{2} \mathrm{O}$ mixtures at different concentrations and temperatures. For ethanol $/ \mathrm{H}_{2} \mathrm{O}$ and DMSO/ $\mathrm{D}_{2} \mathrm{O}$ mixtures $x^{\times}=x^{ \pm}$in the investigated temperature range, while for 1-propanol/ $\mathrm{H}_{2} \mathrm{O}$ $x^{\times} \neq x^{ \pm}$. In the following we discuss the characteristic points of the thermal diffusion behavior and relate them with the properties of the pure components and the mixture.

\subsubsection{The effect of temperature}

The observed independence of the sign change concentration on temperature for DMSO/water mixture is supported by a recent NMR study. Mizuno et al. [97] measured the chemical shift of water hydrogen $\delta_{\mathrm{H}_{2} \mathrm{O}}$, which is related to polarization of water molecules, in dependence of concentration and temperature. In the investigated temperature range between $1-48.5^{\circ} \mathrm{C}$ the chemical shift for pure water is reached around $x_{w} \approx 0.8$. Then the chemical shift slightly overshoots the water value indicating that DMSO stabilizes the water structure. Therefore the stabilized hydrogen bond network forms temperature independent between $x_{w} \approx 0.8$ and 1.0 , suggesting $x^{\times}=x^{ \pm}$in the investigated temperature range. Also for the system acetone/water a stronger polarization of the water molecules in the vicinity of acetone has been observed at high water content $x_{w}>0.96$ [98]. So the polarization effect occurs at higher water content compared to DMSO/water, but it shows in the investigated temperature range no temperature dependence. The thermal diffusion behavior of those two systems follows a similar trend with respect to temperature, whereas the water content at the sign change concentration is higher for acetone/water than for DMSO/water.

Takamuku et al. [162] investigated aqueous solutions at low temperatures by X-ray scattering. They found, that for methanol/water mixture the composition of the dominant clusters formed at $25^{\circ} \mathrm{C}$ does not significantly change when the temperature is lowered. In contrast, 2propanol/water shows a stronger temperature dependence. For 2-propanol the chain clusters are enhanced in the mixture when the temperature is lowered. This might be an indication why for systems with stronger hydrophobic interactions the sign change concentration of the Soret coefficient can be temperature dependent. 


\subsubsection{Relation between the thermal diffusion motion and a struc- tural change in the fluid}

At high water content for all aqueous systems studied so far water accumulates at the warm side. In many cases the Soret coefficient changes its sign at a certain solvent concentration $x_{s}^{ \pm}$. For the systems regarded here we observe the following sequence $x_{s}^{ \pm}=0.08,0.095,0.11,0.14,>$ 0.18 and 0.195 for 1-propanol, 2-propanol, acetone, ethanol, methanol and DMSO in water. In the case of methanol/water the sign change concentration could not be determined precisely from the literature data [164] and also TDFRS measurements were not possible in the entire concentration range due to the low refractive index contrast between methanol and water. Therefore, we estimated the highest concentration of methanol, below which no sign change occurs by measuring diluted aqueous solutions.

It is intuitive to relate the sign change concentration with structural changes in the fluid mixture. There are numerous nuclear magnetic resonance (NMR) [97], mass spectrometry [154], dielectric spectroscopy [143] and x-ray scattering [161] studies, which investigate the change from the tetrahedral structure of water to other structures such as chain-like conformations with increasing solvent concentration. Takamuku et al. [161] investigated alcohol/water solutions by X-ray scattering and found that the structure changes with increasing alcohol concentration in the order of n-propyl $\approx$ iso-propyl $>$ ethyl $>$ methyl at alcohol mole fractions of $0.1,0.1,0.2$, and 0.3 , respectively. Additionally, the same concentrations correspond to the minima of the enthalpies of mixing of the alcohol/water mixtures, which is explained by a compensation of an enthalpic gain due to alcohol/water hydrogen bonding with an enthalpic loss due to a breaking of the tetrahedral water structure with increasing alcohol concentration. Thus, the larger the hydrophobic group, the more rapidly the tetrahedral-like structure of water is disturbed with increasing alcohol concentration. We observed the same tendency for the sign change concentrations for aqueous mixtures of 1-propanol, 2-propanol, ethanol and methanol. The small difference in the thermal diffusion behavior between 1-propanol and 2-propanol might be explained by the observation, that the minimum of the excess enthalpy for 1-propanol/water mixture is shifted more towards the water rich region compared to 2-propanol/water mixture [143], which suggests that the water structure is more effectively disrupted by 1-propanol. The structural changes in DMSO/water mixtures have been investigated by Shin et al. [154] by mass spectrometry. They observed that the water clusters 
disappear between $x_{s}=0.19$ and 0.25 , which is the concentration range, in which the Soret coefficient changes it's sign. The structural change is also supported by the NMR-study by Mizuno et al. [97] which has been discussed in Sec. 6.4.1. They found that the chemical shift of methyl hydrogen decreased below a $x_{w}=0.8$ indicating a disruption of the tetrahedral water structure. This supports our hypothesis that the direction of the thermal diffusion is correlated with the fluid structure - indicated by the formation of water clusters - on a microscopic level.

\subsubsection{The effect of solubility}

The absolute value of the Soret coefficient was previously connected with the solubility parameter (c.f. Chapter 2). Mutually soluble components become undistinguishable, so that the Soret coefficient of such mixtures is equal to zero. Consequently, the Soret coefficient should increase with decreasing solubility. Generally, the solubility is determined by the Gibbs mixing energy $\left(\Delta G_{M}=\Delta H_{M}-T \Delta S_{M}\right)$. With increasing temperature enthalpy $\left(\Delta H_{M}\right)$ and entropy $\left(-T \Delta S_{M}\right)$ contributions to the Gibbs energy act in the opposite way (as far as both $\Delta H_{M}$ and $\Delta S_{M}$ become larger due to weaker hydrogen bonding and less ordering in the system, respectively). For ethanol/water and DMSO/water mixtures the increasing temperature leads probably to an increasing solubility [15], which decreases the absolute values of the Soret coefficient in the whole concentration range (c.f. Fig. 6.4a, 6.5a). This is not the case for water propanol mixtures (c.f. Fig. 6.6). The additional contribution in enthalpy/entropy competition due to the enhancement of propanol clusters [161] could be responsible for the observed behavior (c.f. Fig. 6.6).

\subsubsection{The effect of hydrophilic interactions}

As we already mentioned the sign change concentration can be attributed to the stability of water clusters. At the same time, the stability of water clusters is determined by the hydrophilic (dipole moment) and hydrophobic (number of carbon atoms in hydrophobic part) parts of the solute molecules. In order to study the effect of hydrophilic interactions we consider aqueous mixtures of solvents with the same hydrophobic part (two carbon atoms). In the case of acetone and propionaldehhyde the third carbon is polarized by the double bond 
with the oxygen and not counted. With the assumption that the intermolecular interactions can be characterized by the enthalpy of vaporization, the stability of water clusters can be determined by the ratio of vaporization enthalpy of the solvent and water. The ratio of the vaporization enthalpies can also be attributed to the ratio of pure interaction parameters of a Lennard-Jones (LJ) mixture (e.g. $\psi=H_{2}^{v a p} / H_{1}^{v a p}=\varepsilon_{22} / \varepsilon_{11}$ ) (c.f. Chapter 2). Artola et al. [9] investigated the concentration dependence of the Soret coefficient for binary LJ mixtures of particles with the same masses and sizes but different strength of direct $\psi=\varepsilon_{22} / \varepsilon_{11}$ and cross interaction energies $k_{12}=\varepsilon_{12} \sqrt{\left(\varepsilon_{22} / \varepsilon_{11}\right)}$, respectively. The slope of the composition dependence $S_{\mathrm{T}}(x)$ is controlled by the value of $k_{12}$. This corresponds with a variation of the temperature (c.f. Fig. 6.4a and 6.5a). In contrast $\psi$, which is equivalent to the ratio of vaporization enthalpies, has almost no effect on the slope, but it shifts the Soret coefficient as mass and inertia changes do. The latter was already noticed by Köhler et al. [36]. In Fig. 6.8 the sign change concentration is plotted versus the ratio of the vaporization enthalpies $H_{s}^{\text {vapor }} / H_{w}^{\text {vapor }}$ of the pure components, which is equivalent to the $\psi$ parameter. Data for aqueous solutions of solutes with a similar hydrophobic part (propionaldehyde, acetone, DMSO, ethanol) follow the straight line, which indicates for those systems the importance of hydrophilic interaction rather than effect of mass or moment of inertia. The isotopic substitution of water decrease $x_{s}^{ \pm}$, but the roughly $10 \%$ larger vaporization energy for heavy water compensates this effect so that also those systems follow the line. At the same time decreasing (or increasing) the hydrophobic part of the solute increases in case of methanol (or decrease in case of propanol) the concentration at which the sign change occurs. A similar trend has been observed for the break down of the hydrogen bond network [161]. Additionally, our measurements suggest that for all systems on the line the sign change concentration is temperature independent, $x_{s}^{ \pm}=x^{\times}$.

\subsubsection{Effects in alcoholic mixtures with DMSO}

The system methanol/DMSO stands out from the other systems because it has neither an intersection point nor a point when the Soret coefficient changes its sign. In a recent molecular dynamic simulation by Vechi and Skaf [170] it was shown that the structure formation in methanol/DMSO mixtures is not markedly altered by changing the concentration. This is an indication that DMSO and methanol are quite similar and compatible, which might also be the 


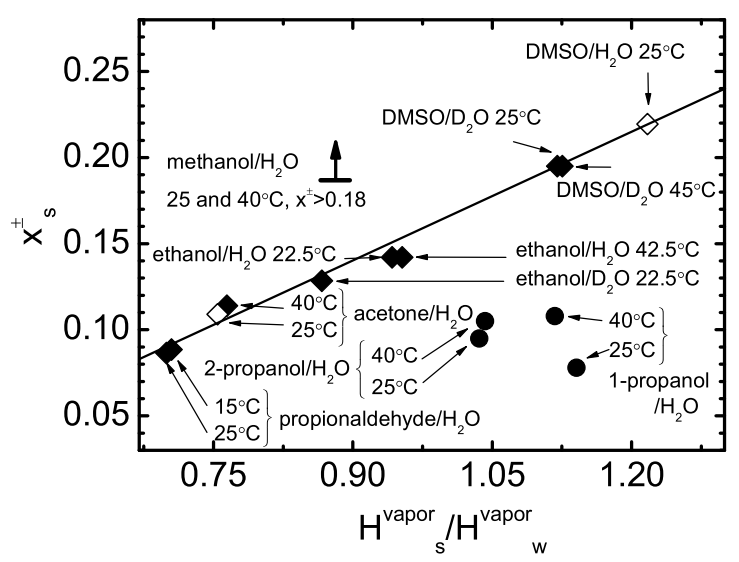

Figure 6.8: Sign change concentration $x_{s}^{ \pm}$plotted versus the ratio of the vaporization enthalpies of the pure components. For aqueous systems where the solvent has two carbons $\diamond) x_{s}^{ \pm}$increases linearly with the ratio $H_{s}^{\text {vapor }} / H_{w}^{\text {vapor }}$, while solvents with three carbon atoms (๑) do not follow the line. Some data for DMSO/ $\mathrm{H}_{2} \mathrm{O}(\diamond)$ and acetone $/ \mathrm{H}_{2} \mathrm{O}(\diamond)$ have been taken from the literature [111].

reason that the sign of the Soret coefficient does not change in dependence of concentration.

The stability of DMSO clusters influences the thermal diffusion behavior of both mixtures. The absence of the sign change for methanol/DMSO mixtures means better miscibility of methanol (no DMSO cluster) in comparison with ethanol (DMSO clusters form) in DMSO. Unfortunately, we did not find a value for the Gibbs energy of mixing, but the enthalpic contribution is negative for methanol/DMSO and positive for ethanol/DMSO mixtures [134], which indicates a better solubility of methanol in DMSO. The difference between the systems methanol/DMSO and ethanol/DMSO mixtures lies in their different hydrophilic groups. As stated already methanol is very similar to DMSO and the fluid structure does not depend on the mixing ratio. The similarity of the two substances is also reflected by the similar hydrogen bonding parameters of DMSO and methanol, which are almost twice as large as the one for ethanol [12]. Therefore it is likely that ethanol will introduce a different fluid 
structure due to the larger hydrophobic group which leads then to a sign change of the Soret coefficient.

\subsection{Conclusion}

We studied systematically the thermal diffusion behavior of associated and highly polar mixtures. Many of those mixtures show a sign change of $S_{\mathrm{T}}$ with concentration. In the case of aqueous mixtures the sign changes occurs at high water content between $x_{w}^{ \pm}=0.8-0.92$. For many systems this concentration can be related with a structural change from a tetrahedral to a chain-like order. Another characteristic point of the investigated systems is the concentration $x_{w}^{\times}$at which the Soret coefficient does not depend on temperature in the investigated range. While for non-associating mixtures $S_{\mathrm{T}}\left(x_{w}^{\times}\right) \neq 0$ the Soret coefficient vanishes often for associating mixtures $\left(S_{\mathrm{T}}\left(x_{w}^{\times}\right) \neq 0\right)$. For those systems with $x_{w}^{ \pm}=x_{w}^{\times}$we found that $x_{w}^{ \pm}$depends linearly on the ratio of the vaporization enthalpies of the pure components. Additionally the hydrophobic part of those systems consists of two carbon atoms.

We also investigated two polar but non-aqueous mixtures: methanol/DMSO and ethanol/ DMSO. The system methanol/DMSO does neither show a sign change nor an intersection point of the isotherms, which might be due to the similarity of methanol and DMSO. Ethanol/DMSO behaves like a typical non-associating mixture with $x_{w}^{ \pm} \neq x_{w}^{\times}$. To reveal further the microscopic mechanism of the thermal diffusion process molecular dynamic studies need to be performed. 


\section{Investigation of the Soret effect by the thermal lens technique}

In this chapter we investigate the thermal diffusion behavior of three different binary mixtures with a thermal lens (TL) setup. In the setup used in this study we avoid the addition of a dye for systems, such as aqueous mixtures, with a weak absorption band at a wavelength of $980 \mathrm{~nm}$. In some aqueous systems with a complex phase behavior the addition of dye significantly affects the apparent measured thermal diffusion properties. The studied systems are dimethylsulfoxide (DMSO) in water, the ionic liquid 1-ethyl-3-methylimidazolium ethylsulfate (EMIES) in butanol and a non-ionic surfactant hexaethylene glycol monododecyl ether $\left(C_{12} E_{6}\right)$ in water. The Soret coefficients of the selected systems cover a range of two orders of magnitude. For DMSO in water with a very low Soret coefficient of the order of $S_{\mathrm{T}} \sim 10^{-3} \mathrm{~K}^{-1}$ we find for a low DMSO content $(c=0.33)$ a reasonable agreement with previous measurements, while the weak thermal lens signal for the DMSO-rich mixture $(c=0.87)$ leads to $20 \%$ too large Soret coefficients with an uncertainty of more than $30 \%$. Secondly we studied a liquid salt 1-ethyl-3-methylimidazolium ethylsulfate (EMIES) in butanol with a roughly ten times higher Soret coefficient of $S_{\mathrm{T}} \sim 10^{-2} \mathrm{~K}^{-1}$. For this system we performed additional measurements with another experimental technique, the classical thermal diffusion forced Rayleigh scattering (TDFRS), which requires the addition of a small amount of dye to increase the absorption. In the entire investigated concentration range the results obtained with the TL and classical TDFRS technique agree within the error bars. As a third system we studied a 
non-ionic surfactant hexaethylene glycol monododecyl ether $\left(C_{12} E_{6}\right)$ in water with a Soret coefficient of the order of $S_{\mathrm{T}} \sim 10^{-1} \mathrm{~K}^{-1}$. For this system we find good agreement with previous measurements. We conclude that the TL technique is a reliable method for systems with a strong optical contrast and fairly large Soret coefficient of the order of $S_{\mathrm{T}} \sim 10^{-2} \mathrm{~K}^{-1}$. *

\subsection{Introduction}

Thermal diffusion describes the migration of molecules in a temperature gradient. As a result of this process a concentration gradient builds up. In the steady state when the mass flux vanishes, the concentration gradient is given by

$$
\nabla c=-S_{\mathrm{T}} c(1-c) \nabla T
$$

where $S_{\mathrm{T}}=D_{\mathrm{T}} / D$ is the Soret coefficient, $D_{\mathrm{T}}$ is the thermal diffusion coefficient, $D$ is the translational diffusion coefficient, $c$ is the weight fraction. A positive Soret coefficient of the component with the weight fraction $c$ implies that this component moves to the cold region.

The main practical applications are separation processes [145, 29] such as thermal field flow fractionation of polymers and colloids or isotope separation, characterization of geochemical processes [66, 30] and combustion [128].

Even less than 20 years ago, different experimental techniques such as thermo gravitational columns, beam deflection, diffusion cells and thermal diffusion forced Rayleigh scattering (TDFRS) gave different results for simple organic mixture such as toluene/n-hexane $[46,75,86]$. The reason for the deviations are manifold, like technical imperfections and the presence of convection. Therefore, a benchmark test has been initiated, to measure thermal diffusion properties of simple organic mixtures by different experimental techniques [117].

The principle of the classical TDFRS method is as follows: a grating created by the interference of two laser beams is written into a sample. Except for the recently developed IR-TDFRS [? ], a small amount of dye present in the sample converts the intensity grating into a temperature grating which in turn causes a concentration grating by thermal diffusion. Both gratings, temperature and concentration, contribute to a refractive index grating, which

* The work described in this chapter is accepted for publication in Phys. Chem. Chem. Phys. 
refracts a third laser beam. The time dependence of the diffracted signal intensity is analyzed and gives the diffusion coefficient $D$, the thermal diffusion coefficient $D_{T}$ and the Soret coefficient $S_{T}$. In the benchmark test it was demonstrated that the classical TDFRS method gives reliable results for organic mixtures and also the data obtained for simple aqueous systems compare well with other experimental techniques [78, 188, 45, 74]. However, recent studies on the surfactant hexaethylene glycol monododecyl ether $\left(\mathrm{C}_{12} \mathrm{E}_{6}\right)$ in water with the classical TDFRS showed, that the small amount of dye added to create a temperature grating, leads to an unexpected second mode in the concentration part of the classical TDFRS signal[110, 109]. The thermal lens (TL) technique is another powerful method which can be used to study the Soret effect in liquid mixtures, ferrofluids and micellar solutions [7, 6, 137, 142]. The basic principle of the TL experiment is that a focused laser beam causes local heating in a sample, which leads in a mixture first to a thermal lens (local refractive index change due to temperature variations) and then to a Soret lens (local refractive index change due to concentration variations). In some of the experiments [142] a small amount of dye is added to achieve a sufficient heating by the laser beam others use a weak absorption band of water in the infrared [137]. First, Gordon et al.[62] observed the thermal lens effect in a liquid placed within the resonator of a helium-neon laser. Later, Giglio and Verdramini [59] noticed that the thermal lens in a binary mixture was noticeably larger than in pure components. The first careful theoretical analysis of the TL effect was done by Norman et al. [112] and Carter et al. [25]. The thermal lens method has been used to study the sign of the Soret coefficient of ferrofluids [7] and to measure $S_{\mathrm{T}}$ for ferrofluids[6] as well as for ionic surfactant systems [137] and Ludox particles in water[137]. The Soret coefficient for Ludox particles in water is only for small Debye lengths consistent with results from classical TDFRS, while for larger Debye lengths $S_{\mathrm{T}}$ determined by TL experiments is significantly larger than in the classical TDFRS [108]. The obtained Soret coefficient for maghemite nanograins coated with negatively charged citrate ions and dispersed in water (ferrofluids) agreed to some extent with measurements obtained by a transient grating technique (deviations are of the order of 20\%) [6]. In contrast, Voit [172] measured 40\% smaller Soret coefficient for benchmark n-dodecane/1,2,3,4 tetrahydronaphtalene mixture. This disagreement can be explained by convection, which results in better mixing, making the Soret coefficient smaller.

In many points the TDFRS and TL setups are comparable. Both techniques are optical 
methods, which rely on the refractive index contrast of the mixture, and, except for a few special cases [34], both methods are limited to binary mixtures. In the TDFRS experiments two lasers are needed with a coherence length of the order of a few centimeters to produce a holographic grating in the sample and to allow for heterodyne signal detection. Additionally, the wavelengths of the two lasers need to be so far apart that the sample absorbs the light at one wavelength, while the sample has to be transparent for the other wavelength. The use of two different wavelengths makes it also more difficult to change the wavelength of the writing beam. In the TL experiment the laser needs to have a stable Gaussian profile with a good stability. The grating vector $q$ is well defined in the TDFRS experiment which enables a detailed analysis of polydisperse systems [76]. In total the equipment requirements and costs are higher for TDFRS than for the TL. In general the equilibration times for the TL is 30times higher than for TDFRS, which makes the TDFRS more suitable for very slow diffusing particles. The weakest point of the TL setup is its sensitivity to convection which can be a problem for slow diffusing systems and systems with a low optical contrast. In the first case convection often sets in before equilibrium is reached. In the latter case a thicker sample cell would be used to increase the contrast, which however leads to enhanced convective flow. Due to the small dimension in the TDFRS experiments convection, is usually not a problem. To check for the occurence of convection TDFRS experiments are often repeated at different powers of the writing beam and the coefficients are extrapolated to a power of zero. Nevertheless, for many systems with a good signal to noise ratio, the TL method is a compact and robust method to measure the thermal diffusion properties of liquid mixtures, polymer solutions and dispersions of small colloidal particles with a radius below $100 \mathrm{~nm}$.

The goal of this paper is to validate the thermal lens technique as a method to measure thermal diffusion properties. The database for reliable Soret coefficients and thermal diffusion coefficients is still very small. As mentioned before the only benchmark test has been performed for organic mixtures [117]. The best studied aqueous system is ethanol/water $[78,188,45,74]$, but the refractive index of ethanol $(n=1.359)$ is very close to that of water ( $n=1.333$ ), so that we expected that it would be difficult or impossible to measure this system with the thermal lens setup. Therefore, we chose dimethylsulfoxide (DMSO), which has a higher refractive index $(n=1.479)$ and it has also been measured before. During the experiments it turned out that the strength of the signal was not sufficient in the entire concentration 
range. We looked for a simple system with a larger Soret coefficient and chose the ionic liquid 1-ethyl-3-methylimidazolium ethylsulfate (EMIES). It was found that EMIES decomposes in the presence of water to form 1-ethyl-3-methylimidazolium hydrogen sulfate and ethanol under ambient conditions [51], so that we used instead of water butanol as solvent, which shows a sufficient absorption in the near infrared. To our best knowledge is the first time that a ionic liquid mixtures has been investigated. As a complex and interesting system we finally investigated the non-ionic surfactant system $\mathrm{C}_{12} \mathrm{E}_{6}$ in water, which also has been investigated before by classical TDFRS [109] and was one of our motivations to build this set-up and the IR-TDFRS [107]. For these three types of mixtures the Soret coefficient $S_{\mathrm{T}}$ differs by orders of magnitudes $\left(10^{-3}, 10^{-2}\right.$ and $10^{-1} \mathrm{~K}^{-1}$, respectively). All three systems show a sufficient absorption at $\lambda=980 \mathrm{~nm}$, so that we do not need to add a dye in the TL experiment. The obtained results for DMSO/water and $\mathrm{C}_{12} \mathrm{E}_{6}$ in water were compared with recent measurements obtained with the classical TDFRS, which needs a small amount of dye to assure a sufficient absorption of the wavelength of the writing beam [111]. In the case of the ionic liquid we performed additional measurements with classical TDFRS, because for this system no literature data are available. The previous measurement for the non-ionic surfactant system with the classical TDFRS showed that the addition of the dye causes changes in the measured thermal diffusion behavior [109]. We compare the TL measurements with recent measurements using IR-TDFRS [107], which works also without dye if the system shows sufficient absorption at $\lambda=980 \mathrm{~nm}$.

\subsection{Experiment and working equations}

\subsubsection{Sample Preparation.}

Hexaethylene glycol monodecyl ether $\left(\mathrm{C}_{12} \mathrm{E}_{6} ; \geq 98 \%\right)$ was ordered from Nikkol Chemicals (Tokyo). Butanol (99.5\%), dimethylsulfoxide (DMSO; 99.7\%) were purchased from Sigma-Aldrich and 1-ethyl-3-methylimidazolium ethylsulfate (EMIES; 99\%) was ordered from Solvent Innovation GMBH. We took deionized water Milli-Q. All chemicals were used without further purification. The aqueous solutions of DMSO were prepared without dye. The EMIES/butanol mixtures for classical TDFRS contain a small amount (roughly $10^{-6} \mathrm{wt}$ $\%$ ) of the dye quinizarin (Aldrich). The amount of the dye corresponds to an optical density 
of $1 \mathrm{~cm}^{-1}$ at a wavelength $\lambda=488 \mathrm{~nm}$, while in the TL experiments no dye was used. The aqueous solutions of the non-ionic surfactant $\mathrm{C}_{12} \mathrm{E}_{6}$ were investigated by the TL method with and without dye. In this case we used the water soluble dye basantol yellow. After the nonionic surfactant $\mathrm{C}_{12} \mathrm{E}_{6}$ had been added to the water the solution was stirred at least for four hours at room temperature.

\subsubsection{Refractive index increment measurements.}

Refractive indices were determined using an Abbe refractometer. The refractive index increment $(\partial n / \partial c)_{p, T}$ at constant pressure and temperature was determined from the derivative of a second order polynomial fit of refractive index data. The temperature derivatives of the refractive index $(\partial n / \partial T)_{p, c}$ at a constant pressure and concentration were determined in a temperature range $T \pm 3^{\circ} \mathrm{C}$ using a Michelson interferometer [14]. The refractive index increments for the binary mixture of DMSO/water and the surfactant solution of $\mathrm{C}_{12} \mathrm{E}_{6} /$ water were taken from Ning et al. [111, 109].

\subsubsection{TDFRS experiment and data analysis}

The classical TDFRS and the IR-TDFRS experiment are described elsewhere in detail [109? ]. An argon-ion laser $\left(\lambda_{w}=488 \mathrm{~nm}\right)$ or infrared laser $\left(\lambda_{w}=980 \mathrm{~nm}\right)$ are used for writing a grating. The laser beam is splitted into two writing beams of equal intensity by a beam splitter. An intensity grating is created in the sample by the interference of these two laser beams. In a classical TDFRS experiments a small amount of dye in the sample converts the intensity grating into a temperature grating. While in the IR-TDFRS the absorption at $\lambda_{w}=980 \mathrm{~nm}$ is utilized to convert the light grating into a temperature grating, which in turn causes a concentration grating by the effect of thermal diffusion. Both gratings contribute to a combined refractive index grating, which is read out by diffraction of a third laser beam $\left(\lambda_{r}=633 \mathrm{~nm}\right)$.

The heterodyne signal intensity $\zeta_{\text {het }}(t)$, normalized to the thermal signal, is related to the Soret coefficient as follows

$$
\zeta_{\text {het }}(t)=1-A\left(1-e^{-q^{2} D t}\right)
$$




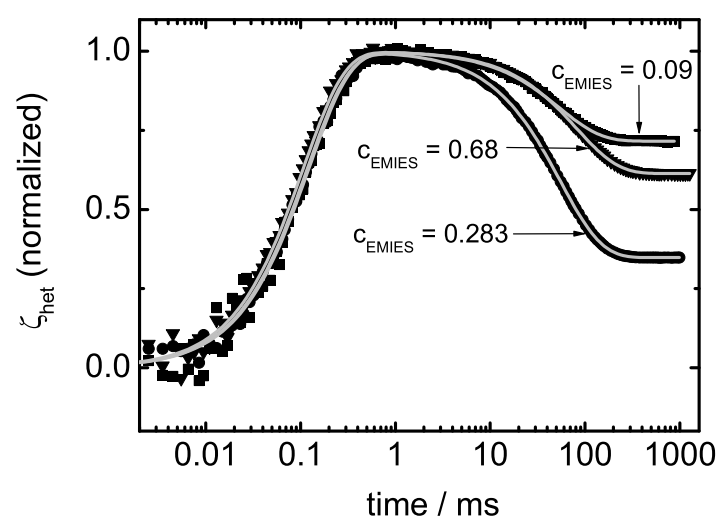

Figure 7.1: Typical normalized classical TDFRS signals of EMIES/butanol mixtures in a cell with $l=0.2 \mathrm{~mm}$ for three different weight fractions $c$ of the ionic liquid EMIES at $30^{\circ} \mathrm{C}$. Solid symbols show the experimental results, lines are the fitted curves according to Eq. 7.2.

with

$$
A=\left(\frac{\partial n}{\partial T}\right)_{p, c}^{-1}\left(\frac{\partial n}{\partial c}\right)_{p, T} c(1-c) S_{\mathrm{T}}
$$

where $q$ is the grating vector, $D$ is the translational diffusion coefficient and $A$ is the amplitude of the concentration signal.

To determine transport coefficients, Eq. 7.2 is fitted to the measured heterodyne signal taking into account the deficiencies of the Pockels cell by an iterative correction algorithm [109] and the two contrast factors $(\partial n / \partial c)_{p, T}$ and $(\partial n / \partial T)_{p, c}$, which are measured separately. Figure 7.1 shows a typical diffraction signal for the ionic liquid mixture EMIES/butanol. For all three concentrations we observe a negative concentration plateau indicating that the ionic liquids EMIES accumulates in the warm region. 


\subsubsection{Thermal lens experiment and data analysis}

\section{Thermal lens effect}

The principle of the TL method is described elsewhere in details $[25,137]$. The TL setup is sketched in Fig. 7.2. The mechanical shutter between the first and the second lens is used for "switching" the laser beam. The focused Gaussian laser beam illuminates a weakly absorbing sample, generating a temperature gradient within a characteristic time $\tau_{\text {th }}$. Later a concentration gradient within a characteristic time $\tau_{\text {Soret }}>>\tau_{\text {th }}$ is induced by the Soret effect. The characteristic times $\tau_{\text {th }}$ and $\tau_{\text {Soret }}$ can be calculated using

$$
\tau_{\mathrm{th}}=\frac{\omega^{2}}{4 D_{\mathrm{t} h}} ; \quad \tau_{\mathrm{Soret}}=\frac{\omega^{2}}{4 D}
$$

where $\omega$ is the beam size at the cell position, $D_{\mathrm{t} h}$ and $D$ are the thermal diffusivity and the translational diffusion coefficient, respectively. The thermal and Soret lenses are formed due to the dependence of the sample refractive index on temperature and concentration, respectively. The resulting time dependence of the beam center intensity can be used in order to estimate the thermal conductivity $\kappa=\rho c_{p} D_{\mathrm{t} h}$ and the Soret coefficient $S_{\mathrm{T}}$. Here, $\rho$ and $c_{p}$ refer to the density and the heat capacity at constant pressure, respectively. In order to measure the intensity in the center of the beam, we place a detector with pinhole at a large distance $\left(d_{\mathrm{s} d}=130 \mathrm{~cm}\right)$ from the sample cell.

\section{Working equations}

In the following section we present the working equations to describe the TL effect. In a binary mixture the thermal lens is created in two steps. First the thermal lens is formed, which is characterized by the time constant $\tau_{t h}$ and the strength of the thermal lens $\theta_{t h}$. Secondly the Soret lens or concentration lens is formed, which is described with an analog formalism. The relevant parameters are the characteristic time constant $\tau_{\text {Soret }}$ and the strength of the Soret lens $\theta_{\text {Soret }}$.

The thermal lens is induced by a Gaussian beam with a power $P$ and a wavelength $\lambda$ in a sample with absorbtion coefficient $b$ and thermal diffusivity $D_{\mathrm{t} h}$. The full expression for the 


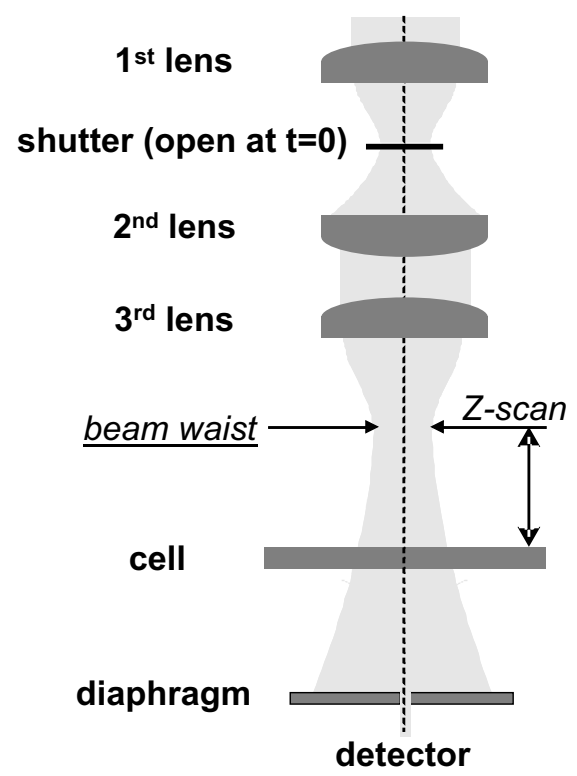

Figure 7.2: Schematic drawing of the TL setup in an upright configuration.

position and time dependence of the beam center intensity response is given by

$$
\begin{array}{r}
I(t)=I(0)\left(1+f\left(\theta_{t h}, \gamma, \tau_{t h}, t\right)\right) ; \\
\text { with } \quad f\left(\theta_{t h}, \gamma, \tau_{t h}, t\right)=A\left(\gamma, \tau_{t h}, t\right) \theta_{t h}+B\left(\gamma, \tau_{t h}, t\right) \theta_{t h}^{2}
\end{array}
$$

The parameter $\theta_{t h}$ characterizes the strength of the thermal lens in the sample and is given by

$$
\theta_{t h}=-\frac{0.52 P b l}{\kappa \lambda} \frac{\partial n}{\partial T}
$$

where $l$ is the cell thickness, $\kappa$ is the thermal conductivity of the sample. The dimensionless parameter $\gamma=\triangle z z_{R}^{-1}$ is the distance from the cell to the beam waist with $\omega=\omega_{0}$ rescaled to the Rayleigh range $z_{R}$, which is the distance between the beam waist and the point with beam radius $\omega=\sqrt{2} \omega_{0}$. The coefficients $A$ and $B$ are equal to,

$$
A\left(\gamma, \tau_{t h}, t\right)=-\operatorname{atan}\left[\frac{2 \gamma}{3+\gamma^{2}+\left(9+\gamma^{2}\right) \tau_{t h} / 2 t}\right]
$$




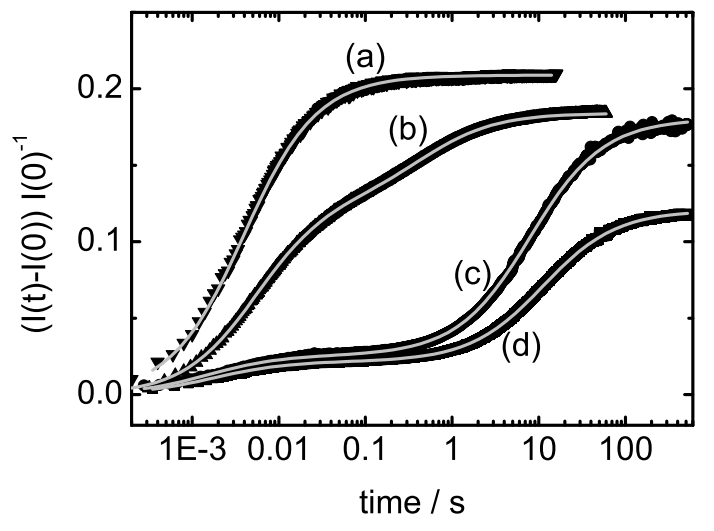

Figure 7.3: Typical TL signals: (a) pure water at $22^{\circ} \mathrm{C}$ in a cell with $l=5 \mathrm{~mm}$; (b) DMSO ( $c=$ $0.33 \mathrm{wt} \%)$ in water $(c=0.67 \mathrm{wt} \%)$ at $25^{\circ} \mathrm{C}$ in a cell with $l=1 \mathrm{~mm}$; (c) $\mathrm{C}_{12} \mathrm{E}_{6}(c=0.1 \mathrm{wt} \%)$ in water $(c=0.9 \mathrm{wt} \%)$ at $30^{\circ} \mathrm{C}$ cell $0.2 \mathrm{~mm}$; (d) $\mathrm{C}_{12} \mathrm{E}_{6}(c=0.05 \mathrm{wt} \%)$ in water $(c=0.95 \mathrm{wt} \%)$ at $30^{\circ} \mathrm{C}$ in a cell with $l=0.2 \mathrm{~mm}$. Solid symbols show the experimental results, lines correspond to the fit using Eq. 7.4 (pure TL effect in case (a)) or with taking into account additional term for describing the Soret effect (c.f. Sec. 7.2.4). 


$$
B\left(\gamma, \tau_{t h}, t\right)=\frac{A^{2}}{4}+\left(\frac{1}{4} \ln \left[\frac{\left[\left(2+\tau_{t h} / t\right)\left(3+\gamma^{2}\right)+6 \tau_{t h} / t\right]^{2}+16 \gamma^{2}}{\left(9+\gamma^{2}\right)\left(2+\tau_{t h} / t\right)^{2}}\right]\right)^{2}
$$

In order to take into account the Soret effect the additional term

$$
f\left(\theta_{\text {Soret }}, \gamma, \tau_{\text {Soret }}, t\right)=A\left(\gamma, \tau_{\text {Soret }}, t\right) \theta_{\text {Soret }}+B\left(\gamma, \tau_{\text {Soret }}, t\right) \theta_{\text {Soret }}^{2}
$$

needs to be added to Eq. 7.4. The Soret coefficient $S_{\mathrm{T}}$ for binary mixture with concentration $c$ and the refractive index derivatives $(\partial n / \partial T),(\partial n / \partial c)$ can be obtained from the ratio of the strength of the Soret lens $\theta_{\text {Soret }}$ and the strength of the thermal lens $\theta_{t h}$ in the sample

$$
S_{T}=-\frac{\theta_{\text {Soret }}}{\theta_{t h}} \frac{\partial n / \partial T}{\partial n / \partial c} c^{-1}(1-c)^{-1}
$$

There are two ways to analyze the experimental data. Typically we fixed the distance between the cell and the beam waist and recorded the time dependence of the intensity $I(t)$, which can be analyzed according to Eq. 7.4. Another way to analyze the data is by calculating the expression $(I(0)-I(t=\infty)) / I(t=\infty)$ with the intial intensity $I(0)$ and the intensity at infinite times $I(t=\infty)$ at different positions. The final expression of this so-called $z$-scan method can also be derived from Eq. 7.4. Both appraoches can be found in the literature $[6,137]$ and they should give the same results. Finally the thermal conductivity $\kappa$ and the Soret coefficient $S_{\mathrm{T}}$ can be calculated according to Eq. 7.5 or 7.9, respectively.

\section{Thermal lens setup}

The schematic diagram of the experimental setup is shown in Fig. 7.2. In order to decrease convection effects our setup has been constructed with an upright optical axis. We have used an infrared laser $(\lambda=980 \mathrm{~nm})$ with a maximum output power of $P=50 \mathrm{~mW}$. The laser was connected with the setup via a monomode fiber. We used a mechanical shutter between the first and the second lens for "switching" the laser beam. The third lens (focal length 100mm) focuses the parallel beam into the sample cell. For all positions along the optical path the cell is mounted perpendicular to the beam. In order to measure the intensity in the center of the beam we place a pinhole with a diameter of $4 \mathrm{~mm}$ in front of the photodiode, which was placed at a distance of $d_{\mathrm{s} d}=130 \mathrm{~cm}$ from the sample cell.

The process of alignment consists of four steps. First, the $x y$-position as well as the angle of the laser beam were adjusted using a CCD camera (Coherent Lasercam HR), which records 
the laser profile. The pinhole was moved along the optical path, while the position of the beam center was monitored by the CCD camera at the same postion, where the photodiode is mounted during the measurement. Secondly, the position of the photodiode with the pinhole has to be adjusted using an infrared viewer. The third step is the optimization of the $x y$ position of each lens, which is done by analyzing the laser profile behind a pinhole, which is placed in the focus of the lens. The same procedure is repeated for each lens with the same pinhole as in the first step. The angle for each lens was adjusted by checking the back reflection on the same pinhole using the infrared viewer. Finally, the cell was slightly tilted to avoid back reflection in the laser diode.

In order to avoid vibrations of the setup during the experiment we mounted the shutter on a separate column and we avoided circulating water for temperature control of the cell. The cell was placed in the copper block, which was heated from both sides by two Peltier elements. The temperature was controlled with an uncertainty $\Delta T= \pm 0.01 \mathrm{~K}$ (Peltron). At the maximum experimental temperature $T=40^{\circ} \mathrm{C}$ the temperature difference between the center and the edge was of the order of $0.05 \mathrm{~K}$. All experiments were performed with a power of typically $P=21 \pm 1 \mathrm{~mW}$. The distance between the cell and the beam waist was $3-4 \mathrm{~mm}$.

\section{Calibration of the thermal lens set-up}

First, we show that we can reproduce the TL signal of pure water with our setup. Fig. 7.3 (curve (a)) shows a typical time dependence of the intensity in the center of the beam for pure water at room temperature. From this dependence the fractional change in the center beam intensity $(I(0)-I(\infty)) / I(\infty)$ at a given distance from the cell to the beam waist can be calculated. The full coordinate dependence of this parameter at different powers for a cell with $l=5 \mathrm{~mm}$ as a function of the distance to the beam waist (called $z$-scan) is shown in Fig. 7.4. The solid lines correspond to the fit according to Eq. 7.4 and 7.5. The inset shows the values of $\theta_{\text {th }}$ calculated from the $z$-scan data at different powers $(P)$ and cell thicknesses $(l)$ versus $P l$. The data are well described with $b=0.5 \mathrm{~cm}^{-1}$ (Ref. 137), $\partial n / \partial T=-0.937810^{-4}$ $\mathrm{K}^{-1}$ (Ref. 74) and $\lambda_{\text {laser }}=980 \mathrm{~nm}$ according to Eq. 7.5. The heat conductivity was found to be equal $0.6098 \mathrm{Wm}^{-1} \mathrm{~K}^{-1}$, which is quite close to the tabulated value $0.603 \mathrm{Wm}^{-1} \mathrm{~K}^{-1}$ in Ref. 186.

For pure butanol at $30^{\circ} \mathrm{C}$ the heat conductivity was determined from the time depen- 


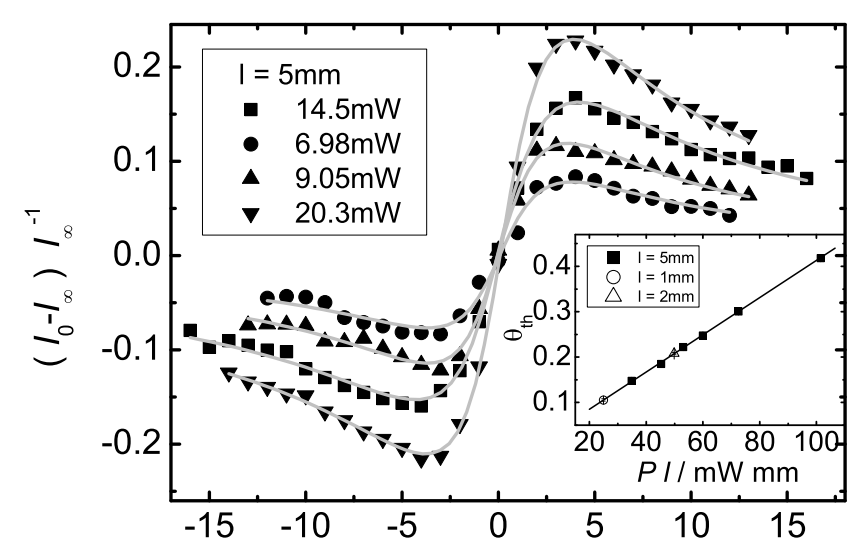

$\Delta \mathrm{z} / \mathrm{mm}$

Figure 7.4: Typical $z$-scan curves for pure water in cell with $l=5 \mathrm{~mm}$ at $22^{\circ} \mathrm{C}$ for different laser powers. These curves are fitted using Eq. 7.4 (c.f. Sec. 7.2.4). The inset shows the thermal lens number $\theta_{t h}$ obtained from $z$-scan data at different laser powers $P$ and cell thicknesses $l$ as a function of the product $P l$. Straight line is according to Eq. 7.5.

dence of the central beam intensity. The obtained value $0.1542 \mathrm{Wm}^{-1} \mathrm{~K}^{-1}(l=1 \mathrm{~mm}$, $P=20.05 \mathrm{~mW}, \theta_{\mathrm{th}}=0.1784, b=0.066 \mathrm{~cm}^{-1}$ and $\left.\partial n / \partial T=-3.9210^{-4} K^{-1}\right)$ is also in good agreement with the reference value $0.153 \mathrm{Wm}^{-1} \mathrm{~K}^{-1}$ in Ref. 186.

\section{Convection effects in the thermal lens experiment}

Generally speaking, the non-zero extinction coefficient of our sample and the finite thickness of the cell lead to a temperature inhomogeneity in the direction parallel to the laser beam. The behavior of the components with different densities becomes sensitive to gravity, which is commonly known as convection. In order to avoid convection, the characteristic equilibration time should be smaller than the characteristic convection time $\left(\tau_{\text {convection }}\right)$. The convection time can be estimated through the ratio of the beam size at the cell position $(\omega \approx 54 \mu \mathrm{m}$ for a typical $\tau_{t h} \approx 5 \mathrm{~ms}$ for a cell with $1 \mathrm{~mm}$ with water, placed $4 \mathrm{~mm}$ before the beam waist; (c.f. Eq. 7.3) to the convection velocity $U_{\text {convection }}$. Rusconi et al.[137] proposed two expressions 
for scaling the convection velocity (c.f. Eq. 7.10 and 7.11).

$$
\begin{aligned}
& U_{\text {convection }} \sim g \alpha l^{2} \triangle T v^{-1} ; \quad G r \ll 1 ; \\
& U_{\text {convection }} \sim(g \alpha v l \triangle T)^{0.5} ; \quad G r \gg 1,
\end{aligned}
$$

where $\alpha$ is the thermal expansion coefficient, $v$ is the kinematic viscosity, $g$ is gravitational acceleration and $\Delta T$ is the characteristic amplitude of the temperature inhomogeneity. The Grashof number $G r$ is defined as

$$
G r=g \alpha \triangle T l^{3} v^{-2}
$$

Eq. 7.10 is only valid for low Grashof numbers $(G r \ll 1)$, while for large Grashof numbers $((G r \gg 1))$ Eq. 7.11 needs to be used.

Rusconi et al.[137] calculated the convection velocity profiles in a cell with $l=0.5 \mathrm{~mm}$ and for a laser power of $P=20 \mathrm{~mW}$ using the Navier-Stokes equations. The maximum value in the center of the cell was found to be $U_{\text {convection }} \approx 2.410^{-6} \mathrm{~m} \mathrm{~s}^{-1}$. Scaling this value according to Eq. 7.10 with the cell thicknesses allows to calculate convection times for different cell thicknesses and different solvents. The obtained values are presented in Table 7.1. The characteristic temperature difference $(\Delta T)$ between the sample temperature at the center of the beam and the average sample temperature were calculated according to the criteria $\Delta T \approx 0.3 P b l \kappa^{-1}$, proposed by Gordon et al.[62]. The corresponding values are also presented in Table 7.1 .

\subsection{Results and Discussion}

\subsubsection{Nonionic surfactant $\mathrm{C}_{12} \mathrm{E}_{6}$ in water}

Fig. 7.3 (curves (c) and (d)) shows typical TL signals obtained in a cell with $l=0.2 \mathrm{~mm}$ of $\mathrm{C}_{12} \mathrm{E}_{6}(c=0.1 \mathrm{wt} \%)$ and of $\mathrm{C}_{12} \mathrm{E}_{6}(c=0.05 \mathrm{wt} \%)$ in water at $30^{\circ} \mathrm{C}$. In the entire range the fit shows no systematic deviations and the obtained values of the Soret coefficient at different concentrations $(c=0.005,0.015,0.025,0.05$ and $0.1 \mathrm{wt} \%)$ and temperatures $(T=30$ and $40^{\circ} \mathrm{C}$ ) agree within the error bars with the Soret coefficients recently obtained by Ning et al. 
Table 7.1: Characteristic convection times for different solvents calculated from convection velocity[137] in pure water in a cell with $l=0.5 \mathrm{~mm}$ at $P=20 \mathrm{~mW}$ for our experimental conditions using Eq.7.10, which is valid for low Grashof numbers $(G r \ll 1)$ ).

\begin{tabular}{|c|c|c|c|c|}
\hline solvent & $\begin{array}{c}\text { temperature } \\
\text { difference } \\
\Delta T / \mathrm{K}\end{array}$ & $\begin{array}{c}\text { cell } \\
\text { thickness / } \\
\text { mm }\end{array}$ & $\begin{array}{c}G r \\
\text { number }\end{array}$ & $\tau_{\text {convection }}$ \\
\hline \multirow[b]{2}{*}{ water } & \multirow[b]{2}{*}{0.49} & 0.2 & $7.610^{-3}$ & 141 \\
\hline & & 1 & $9.510^{-1}$ & $>6$ \\
\hline \multirow{2}{*}{ butanol } & \multirow[b]{2}{*}{0.26} & 0.2 & $2.110^{-3}$ & 164 \\
\hline & & 1 & $2.610^{-1}$ & $>7$ \\
\hline \multirow[b]{2}{*}{ DMSO } & \multirow[b]{2}{*}{0.14} & 0.2 & $2.610^{-3}$ & 218 \\
\hline & & 1 & $3.210^{-1}$ & $>9$ \\
\hline
\end{tabular}

with the IR-TDFRS [107] (c.f. Fig. 7.5). The maximal deviation between IR-TDFRS and TL data is of the order of $14 \%$, but no systematic trend could be observed. The characteristic plateaus at large times (c.f. Fig 7.3) indicate that convection effects are negligible, despite that the typical equilibration times of $\sim 100-200 \mathrm{~s}$ are of the order of the characteristic convection time of $\sim 140$ s (c.f. Table 7.1).

Figure 7.6 shows the Soret coefficient for $\mathrm{C}_{12} \mathrm{E}_{6}(c=0.025 \mathrm{wt} \%)$ in water for different dye contents (basantol yellow) at $40^{\circ} \mathrm{C}$. The influence of the dye is found to be crucial. Increasing the optical density from 0 to $2 \mathrm{~cm}^{-1}$ (typical condition for classical TDFRS measurements) makes $S_{\mathrm{T}} 35 \%$ smaller. According to our previous studies [109, 107] the addition of basantol yellow shifts the two-phase boundary towards higher temperatures. This is the main reason that the Soret coefficient becomes smaller with increasing dye content. At the same time we did not observe a second mode in TL nor in the IR-TDFRS experiment. Such a second mode hat been observed previously with the classical TDFRS [109]. The reason for the second mode in the classical TDFRS is probably some kind of feedback mechanism, which leads to an inhomogenous dye distribution in the sample [107]. 


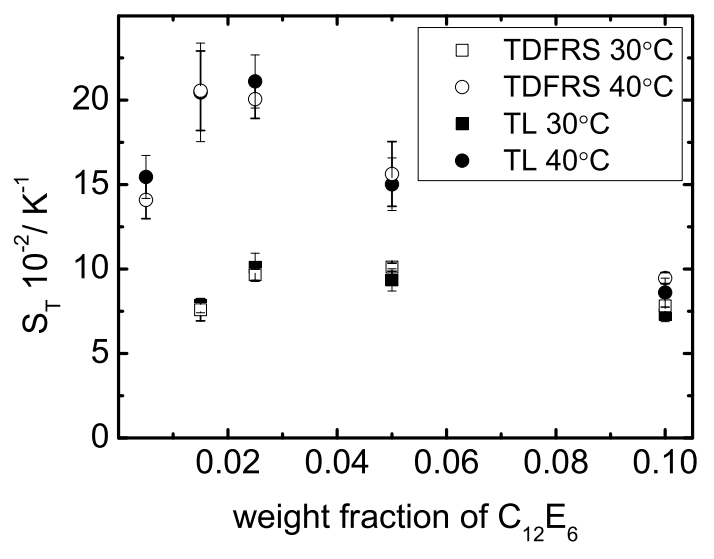

Figure 7.5: Soret coefficient of $\mathrm{C}_{12} \mathrm{E}_{6}$ aqueous solutions at 30 and $40^{\circ} \mathrm{C}$ as a function of surfactant concentration. Open symbols are IR-TDFRS data[107], solid symbols refer to data from TL.

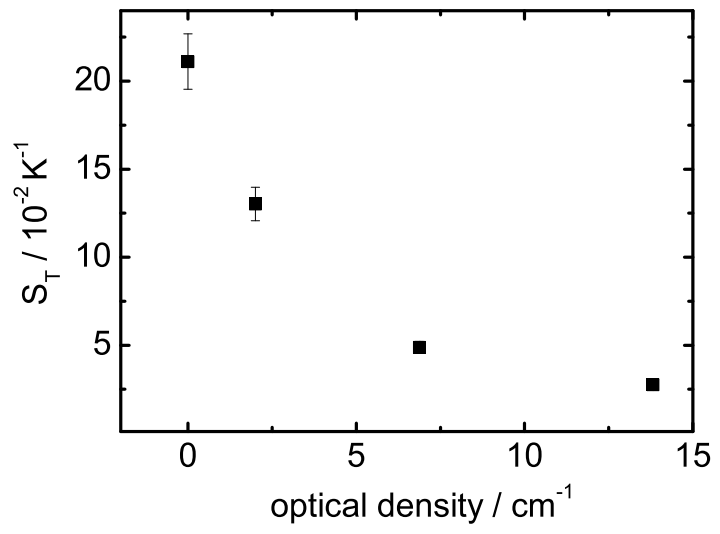

Figure 7.6: Soret coefficient of $\mathrm{C}_{12} \mathrm{E}_{6}\left(c=0.025 \mathrm{wt} \%, T=40^{\circ} \mathrm{C}\right)$ in water measured with the TL method in a cell with $l=0.2 \mathrm{~mm}$ as a function of the dye optical density. 


\subsubsection{DMSO in water}

The Soret coefficient for DMSO/water is roughly two orders of magnitude smaller than for $\mathrm{C}_{12} \mathrm{E}_{6}$ /water mixtures. In the case of an extremely weak thermodiffusion effect, for example close to a concentration, where $D_{\mathrm{T}}$ changes sign, one can expect that the Soret lens will be very weak and difficult to observe. Also thermal fluctuations and other sources of noise such as the stability of the laser will become important. Generally speaking, the sensitivity of the TL setup is associated with the amplitude $A$. If one compares both analysis equations Eq. 7.2 and 7.9 for TDFRS and TL, respectively, both methods are quite similar. The amplitude $A$ in TDFRS is equivalent to the ratio of $\theta_{\text {Soret }}$ to $\theta_{t h}$ in the TL method.

In order to have strong signal we chose two DMSO/water concentrations $(c=0.33$ and $0.87)$ for which the amplitudes of the TDFRS signal are maximal $(0.47$ and 0.12 , respectively). At the low DMSO content, DMSO moves to the cold side, while at higher DMSO content DMSO moves to the warm side. The typical TL signal for a mixture with a water mass fraction of 0.67 in a $1 \mathrm{~mm}$ cell is shown in Fig.7.3. The typical equilibration time for a $1 \mathrm{~mm}$ cell is of the order of the convection time (c.f. Table 7.1).

The measured Soret coefficient in the water-rich region is $S_{\mathrm{T}}=(2.5 \pm 0.4) 10^{-3} \mathrm{~K}^{-1}$ for a $0.2 \mathrm{~mm}$ cell and $S_{\mathrm{T}}=(2.69 \pm 0.35) 10^{-3} \mathrm{~K}^{-1}$ for a $1 \mathrm{~mm}$ cell are in good agreement with the classical TDFRS data with $S_{\mathrm{T}}=(2.7 \pm 0.04) 10^{-3} \mathrm{~K}^{-1}$. The typical relative uncertainties of the TDFRS data are in the 2-3\% range, while the TL data show 5 times higher noise.

In the DMSO-rich region it was not possible to measure reliable values in the $1 \mathrm{~mm}$ cell. The obtained Soret coefficient $S_{\mathrm{T}}=-(3.85 \pm 1.3) 10^{-3} \mathrm{~K}^{-1}$ is $18 \%$ larger than the value of $S_{\mathrm{T}}=(3.23 \pm 0.06) 10^{-3} \mathrm{~K}^{-1}$ obtained in the classical TDFRS. The uncertainty of the TL measurments exceeds $30 \%$, while the uncertainty of the classical TDFRS measurements is of the order of $2 \%$. In order to minimize convection effects we also performed measurements in the cell with $l=0.2 \mathrm{~mm}$. In the thin cell, however, the concentration plateau was often not clearly seen and reproducible measurements were not possible. Under these conditions the Soret lens is too weak. Compared to measurements in the water-rich region the amplitude is 4 times smaller. The relative change in concentration $(\delta c / c)$ due to the temperature gradient was found to be $(\delta c / c) \approx 8 \cdot 10^{-5}$, which is one order of magnitude smaller than the corresponding value of $(\delta c / c) \approx 7 \cdot 10^{-4}$ in the water-rich region. With the DMSO/water system we reach the limits of our thermal lens experiment. The typical noise level $\delta I / I$ in the TL 


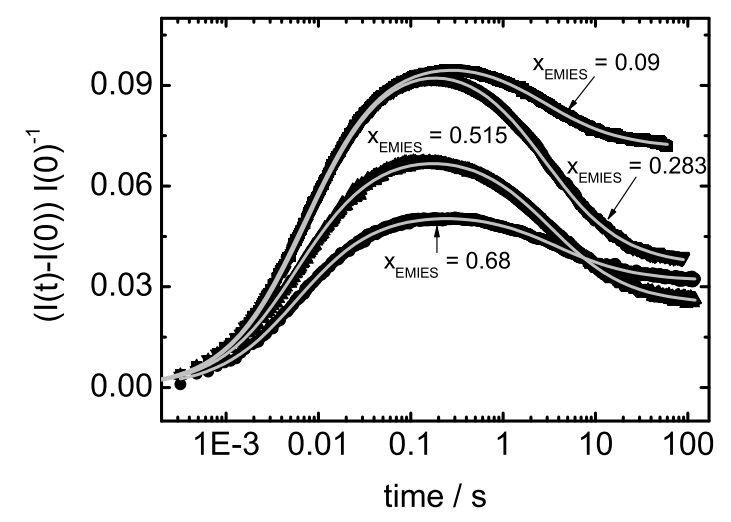

Figure 7.7: Typical TL signals (cell $1 \mathrm{~mm}$ ) for solutions of EMIES in butanol at $30^{\circ} \mathrm{C}$. Solid symbols show the experimental results, lines are the fitting curves according to Eq. 7.4 (pure TL effect) with taking into account the additional term that describes the Soret effect (c.f. Sec. 7.2.4).

experiment is of the order of $1 \%$ leading to an uncertainty in the order of $10 \%$ to the concentration signal. This uncertainty of course influences the stability of the weak Soret lens. With our experimental equipment it was not possible to reduce the main reasons for noise such as vibrations, fluctuations in the intensity and temperature fluctuations further. Under our experimental conditions the signal needs to be at least comparable with one in the water-rich region with weaker noise and stronger Soret lens. Another possibility would be to increase the number repetitions in the TL experiment in order to reduce the error of the mean further. Here we need to keep in mind that an averaging of 2000-4000 measurements is unrealistic due to the roughly 100 times longer equilibration times in the TL experiment as compared to the TDFRS experiment.

\subsubsection{Ionic liquid EMIES in butanol}

The thermal diffusion behavior of EMIES/butanol mixtures was investigated in two different cells with $l=0.2 \mathrm{~mm}$ and $l=1 \mathrm{~mm}$. Fig. 7.7 shows the typical TL signal for the mix- 


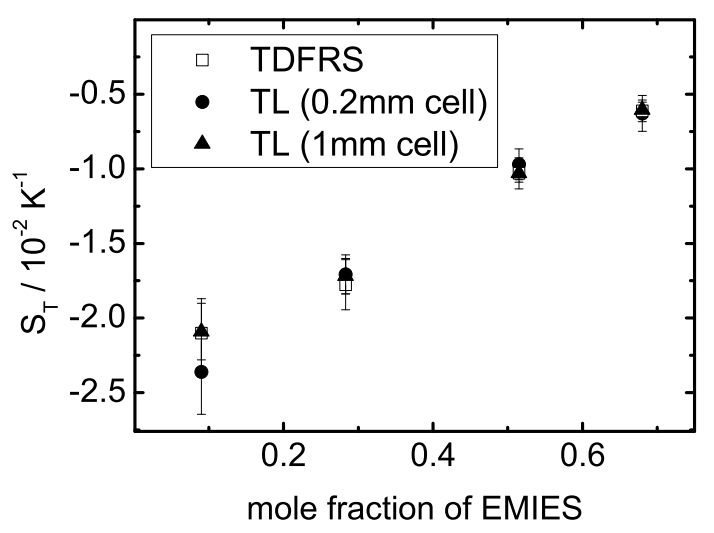

Figure 7.8: Soret coefficient of EMIES in butanol at $30^{\circ} \mathrm{C}$ as a function of concentration. Open symbols are classical TDFRS data, solid symbols refer to TL data $0.2 \mathrm{~mm}$ and $1 \mathrm{~mm}$ cells.

ture EMIES/ butanol $(l=1 \mathrm{~mm})$. The characteristic equilibration time of $\sim 10-15 \mathrm{~s}$ is again of the order of the convection time (c.f. Table 7.1). The fitted function decribes the data well and the residuals are statistically distributed. The obtained Soret coefficients for different cells of varying thickness $(l=0.2$ and $1 \mathrm{~mm})$ for different EMIES concentrations ( $c=0.09,0.283,0.515$ and 0.68$)$ at $T=30^{\circ} \mathrm{C}$ agree within the error bars with the classical TDFRS data. For the lowest salt concentration the maximum deviation found was 19\%, but typically the values agree within $3-7 \%$. The Soret coefficient is of the order of $10^{-2}$ $\mathrm{K}^{-1}$, which is less than the characteristic value $\left(10^{-1} \mathrm{~K}^{-1}\right)$ for $\mathrm{C}_{12} \mathrm{E}_{6} /$ water and larger than $10^{-3} \mathrm{~K}^{-1}$ for DMSO/water mixtures. For the smallest value of $S_{\mathrm{T}}$ at the mass fraction of 0.68 , the relative change in concentration due to the Soret effect $(\delta c / c) \approx 3.4 \cdot 10^{-4}$ is still larger than in case of solution of water in DMSO $\left((\delta c / c) \approx 8 \cdot 10^{-5}\right)$. 


\subsection{Conclusion}

In the present chapter we compared the Thermal Diffusion Forced Rayleigh Scattering (TDFRS) technique with the Thermal Lens (TL) method. We investigated three different systems with Soret coeffcients between $S_{\mathrm{T}} \sim 10^{-3}-10^{-1} \mathrm{~K}^{-1}$. For the systems with the larger Soret coefficients we found good agreement between the two methods. For low Soret coefficients of the order of $S_{\mathrm{T}} \sim 10^{-3} \mathrm{~K}^{-1}$, the TL method reaches its limits. In the case of a low Soret coefficient and a low optical contrast reliable measurements are not possible. Typically slow diffusing molecules such as polymers and colloids have fairly large Soret coefficient of the order of $S_{\mathrm{T}} \sim 10^{-1} \mathrm{~K}^{-1}$, so that they are in principle good systems to be investigated by TL. The other limit of the TL experiment is that the characteristic equilibration time should be smaller than the characteristic convection time. Therefore the investigation of large colloids in the micron scale will be impossible.

A big advantage of the TL method is that it is fairly fast and the experimental setup is much cheaper compared to the TDFRS setups. It is also fairly easy to change the wavelength, so that the addition of dye can be avoided by using the natural absorption of the molecules. An important requirement on the laser source is an excellent Gaussian profile and laser stability, but the coherence length can be very short. If one plans future benchmark tests which should also include the thermal lens method, the systems have to be selected carefully so that the signal to noise ratio is also large enough to do precise TL experiments. According to our experiments ionic liquids such as EMIES in butanol seems to be a good candidate. The Soret coefficient is one order of magnitude larger than that for ordinary molecular sytems, which results in a sufficient signal to noise ratio in the TL experiment, while diffusion is still fast enough so that it is also accessible for other experimental techniques such as thermogravitational columns. 


\section{Summary}

The Soret effect was discovered more than 150 years ago and has been studied in the meantime in gaseous mixtures, mixtures of simple molecules, polymer solutions and colloidal dispersions. The studies cover three main directions: experimental measurements, computer simulations and theoretical predictions of the Soret coefficient. Some progress has been achieved with theoretical calculation of $S_{\mathrm{T}}$ for gas mixtures with Chapman-Enskog theory $[63,173]$. The reasonable agreement with experiment was found. On the other hand, for the mixtures of simple molecules typically it is not even possible to predict the correct sign of $S_{\mathrm{T}}$ due to the lack of accuracy of the chosen equation of state (EOS), calibrated mainly for use in the chemical and petroleum industry [11]. Several rules of thumb were proposed for simple liquid mixtures. It was found that the Soret effect is a function of the difference in mass, size, moment of inertia and vaporization enthalpy of the two mixing partners. Precise phenomenological expressions for $S_{\mathrm{T}}$ were proposed for mixtures of Lennard-Jones particles in RNEMD simulations $[126,56]$ and for mixtures of benzene in cyclohexane in experiment [36], which are useful for simple molecular mixtures.

In this thesis we studied the thermal diffusion behavior of binary mixtures of spherical, chain-like and associated molecules. Experiments were performed using the thermal diffusion Rayleigh scattering technique (TDFRS) and the thermal lens method (TL). For some of the mixtures we calculated the Soret coefficient using reverse non-equilibrium molecular dynamic simulations (RNEMD). Obtained results were analyzed in the framework of thermodynamic theories, particular attention has been given to comparison with other experimental techniques.

In Chapter 2 and 3 we studied the Soret effect in binary mixtures of the component with similar shape (spherical and chain-like molecules) by varying systematically the properties of the mixing partners. More specifically, in Chapter 2 we study the thermal diffusion behavior of three binary mixtures (tetraethylsilane, di-tert-butylsilane and carbon tetrabromide in carbon tetrachloride) consisting of spherical molecules by the TDFRS method. Additionally we applied reverse non-equilibrium molecular dynamics calculations to determine the Soret coefficients for two of the experimentally investigated mixtures. We found a fairly good agree- 
ment between the simulated and experimentally determined Soret coefficients for the systems tetraethylsilane and di-tert-butylsilane in carbon tetrachloride. Although the magnitude of the simulated $S_{\mathrm{T}}$-values is systematically by $9-18 \%$ smaller than in experiment, we found in the experiment and in the simulations that di-tert-butylsilane accumulates slightly stronger in the warm region than the more symmetric tetraethylsilane. The observed thermophoretic motion for those three unpolar mixtures follows the common rules, which state that the component with the larger molar mass, smaller diameter and the larger Hildebrandt parameter accumulates in the cold region. In Chapter 3, we studied systematically binary mixtures of non-spherical n-alkane molecules by two different techniques, a convective method as the thermogravitational columns (TC) (paralelepipedic and cylindrical configurations) and the non-convective TDFRS. In general we found a very good agreement between these two methods. Nevertheless, there are some discrepancies with recently published TC data [85]. Neither the TC nor our TDFRS measurements showed the upward trend of the thermal diffusion coefficient for higher alkanes, which was recently observed. We assume that reason for this disagreement is the relative big gap $L_{x}$ in the TC cells [85] compared to the cells used in this work. The bigger gap decreases the accuracy of determining the mass separation between the two ends of the TC. This is especially important for the mixtures of decane, with the higher alkanes. Our measurements indicate also that the heavier component moves to the cold side as in the case of the mixtures of spherical molecules.

In the Chapter 4 and 5, we systematically studied the influence of the chain-length and the degree of branching on the thermal diffusion behavior of linear and branched alkanes in disk-like benzene with TDFRS, lattice model calculations and RNEMD. In Chapter 4 we have shown experimentally that the Soret effect in alkane/benzene mixtures depends on the molecular mass and structure of the alkane as well as the temperature and composition of the mixture. The Soret coefficient $S_{\text {T }}$ of the alkane was found to be negative for these $n$ alkane/benzene mixtures indicating that the alkanes are enriched in the warmer regions of the liquid mixtures. The magnitude of the Soret coefficient decreases with increasing chain length and increasing alkane content of the mixtures. The temperature dependence of the Soret coefficient depends on mixture composition and alkane chain length; the slope of $S_{\mathrm{T}}$ versus temperature changes from positive to negative with increasing chain length at intermediate compositions. In order to study the shape effect on the Soret effect, mixtures of branched alkanes 
in benzene were also considered. Our results for the Soret coefficients show that the tendency for the alkanes to move to the warmer regions of the fluid decreases with increasing degree of branching. The branching effect is so strong that for 2,2,4-trimethylpentane/benzene mixtures the Soret coefficient changes sign at high alkane content and that equimolar 2,2,3trimethylbutane/benzene mixtures have positive Soret coefficients in the investigated temperature range. For the linear chains, a simple lattice model from Luettmer Strathmann [90] is able to reproduce the experimental trends. For the branched isomers, however, an interpretation of the data is more difficult. A comparison of the experimental data for branched heptane isomers with those for the linear chains between heptane and heptadecane shows that the effect of branching on the Soret coefficients is larger than that of the molecular weight. This is not expected from the thermodynamic properties of the pure alkane fluids. For the branched heptanes, the moments of inertia, calculated about the symmetry axis increase with increasing number of methyl groups as much as the Soret coefficient. In order to clarify the effect of the molecular shape on $S_{\mathrm{T}}$, in Chapter 5 we applied a equilibrium molecular dynamics and the reverse nonequilibrium molecular-dynamic algorithm to calculate the mutual diffusion, tracer diffusion and Soret coefficients in different heptane/benzene mixtures. The heptane/benzene mixture was investigated at different concentrations and the effect of branching was investigated for equimolar mixtures of the branched heptanes (3-MH, 2,3-DMP, 2,4-DMP) in benzene. The simulated Soret and mutual diffusion coefficients show the same trend as in experiment. However, the simulated values of $S_{\mathrm{T}}$ values are systematically $\approx 3 \times 10^{-3} \mathrm{~K}^{-1}$ smaller than in the experiment. The observed decreasing of the magnitude of $S_{\mathrm{T}}$ for equimolar heptane/benzene mixtures can not be explained by mass and size effects. The effect of the molecular shape, which affects the liquid structure, as well as kinetic properties of the mixture, needs to be considered additionally.

In Chapter 6 we systematically studied the thermal diffusion behavior of different associated binary mixtures of water, deuterated water, dimethyl sulfoxide (DMSO), methanol, ethanol, acetone, methanol, 1-propanol, 2-propanol, propionaldehyde. The sign changes of $S_{\mathrm{T}}$ occurs at high water content between $x_{w}^{ \pm}=0.8-0.92$. For many systems this concentration can be related with a structural change from a tetrahedral to a chain-like order in the liquid mixture. Another characteristic point of the investigated systems is the concentration $x_{w}^{\times}$at which the Soret coefficient does not depend on temperature in the investigated range. 
While for non-associating mixtures $S_{\mathrm{T}}\left(x_{w}^{\times}\right) \neq 0$ the Soret coefficient vanishes often for associating mixtures $\left(S_{\mathrm{T}}\left(x_{w}^{\times}\right)=0\right)$. For those systems with $x_{w}^{ \pm}=x_{w}^{\times}$we found that $x_{w}^{ \pm}$depends linearly on the ratio of the vaporization enthalpies of the pure components. Additionally the hydrophobic part of those systems consists of two carbon atoms. For aqueous mixtures of methanol and propanols the sign change concentration is out of the linear trend and correlates with the size of the solute hydrophobic part. We also investigated two polar but non-aqueous mixtures: methanol/DMSO and ethanol/DMSO. The system methanol/DMSO does neither show a sign change nor an intersection point of the isotherms, which might be due to the similarity of methanol and DMSO. Ethanol/DMSO behaves like a typical associating mixture with $x_{w}^{ \pm} \neq x_{w}^{\times}$.

In the last Chapter 7 we compared the thermal diffusion forced Rayleigh scattering technique with the thermal lens method. We investigated three different systems with Soret coeffcients between $S_{\mathrm{T}} \sim 10^{-3} \mathrm{~K}^{-1}$ and $S_{\mathrm{T}} \sim 10^{-1} \mathrm{~K}^{-1}$. For the systems with the larger Soret coefficients we found good agreement between the two methods. For low Soret coefficient in the order of $S_{\mathrm{T}} \sim 10^{-3} \mathrm{~K}^{-1}$ the TL method reaches its limits. In the case of a low Soret coefficient and a low optical contrast reliable measurements are not possible. Typically slow diffusiving molecules such as polymers and colloids have fairly large Soret coefficient in the order of $S_{\mathrm{T}} \sim 10^{-1} \mathrm{~K}^{-1}$, so that they are in principle good systems to be investigated by TL. The other limit of the TL experiment is that the characteristic equilibration time should be smaller than the characteristic convection time. Therefore, the investigation of large colloids in the micron scale will be impossible. The big advantage of the TL method is that it is fairly fast and the experimental setup is much cheaper compared to the TDFRS. An important requirement on the laser source is an excellent gaussian profile and laser stability, but the coherence length can be very short. If one plans future benchmark tests which should also include the thermal lens method, the systems have to be selected carefully so that the signal to noise is large enough to do precise TL experiments. It is also fairly easy to change the wavelength, so that the addition of dye can be avoided by using the natural absorption of the molecules. It turned out that the ionic liquids, which we investigated with the TL method is a good candidate to validate the TL setup and can also be studied with other experimental methods. 


\section{Samenvatting}

Het Soret effect is ongeveer 150 jaar geleden ontdekt en is sindsdien bestudeerd voor gas mengsels, mengsels van eenvoudige molekulen, oplossingen van polymeren en kolloidale dispersies. Deze studies omvatten experimenten, computer simulaties als mede ook theoretische voorspellingen voor de Soret koefficient $S_{\mathrm{T}}$. Enige vooruitgang is geboekt met de berekening van $S_{\mathrm{T}}$ voor gas mengsels met behulp van Chapman-Enskog theorie [63, 173]. Anderzijds is het nog steeds niet mogelijk om zelfs ook maar het korrekte voorteken van $S_{\mathrm{T}}$ voor mengsels van simpele molekulen te voorspellen, voornamelijk door het gebrek aan nauwkeurige toestandsvergelijkingen. Deze zijn over het algemeen gekalibreerd voor gebruik in de chemische en petroleum industrie [11]. Diverse vuistregels zijn voorgesteld voor vloeistof mengsels van simpele molukulen. De Soret koefficient is een functie van het verschil in massa, grootte, traagheidsmoment en de verdampings enthalpie van de twee komponenten. Nauwkeurige fenomenologische uitdrukkingen voor $S_{\mathrm{T}}$ zijn voorgesteld voor mengsels van Lennard-Jones deeltjes, uitgaande van "omgekeerde niet-evenwichts molekulaire dynamica" (ONEMD) simulaties [126, 56], en voor mengsels van benzeen en cyclohexaan op basis van experimenten [36], welke eveneens bruikbaar zijn voor mengsels van simpele molekulen. In deze dissertatie bestuderen we het thermodiffusieve gedrag van bolvormige, keten achtige en geassocieerde molekulen. De experimentele techniek die we hierbij gebruiken is de "thermische diffusie Rayleigh verstrooings" (TDRV) techniek en de "thermische lens" (TL) methode. Voor enkele mengels berekenen we de Soret koefficient middels "omgekeerde nietevenwichts molekulaire dynamica" simulaties. De resultaten worden geanalyseerd binnen het raamwerk van thermodynamische theorien. Resultaten worden eveneens vergeleken met die verkregen m.b.v. andere methoden. In de hoofdstukken 2 en 3 bestuderen we de Soret koefficient voor binaire mengsels van molekulen die dezelfde vorm hebben (bolvormige en keten molekulen), waarbij de eigenschappen van de komponenten systematisch worden veranderd. In het bijzonder, in hoofdstuk 2 , bestuderen we het thermo diffusieve gedrag van drie binaire mengsels bestaande uit bolvormige molekulen (tetra ethyl silaan, di-tert-butyl silaan en koolstof tetrabromide in koolstof tetra chloride), middels de TDRV techniek. Voor twee van deze mengsels hebben we eveneens ONEMD simulaties uitgevoerd, en vergeleken met de 
experimentele resultaten. We vinden een redelijke overeenstemming tussen de gesimuleerde en experimenteel bepaalde Soret koefficienten voor tetra ethyl silaan en di-tert-butyl silaan in koolstof tetra chloride. De grootte van gesimuleerde waarden voor $S_{\mathrm{T}}$ zijn systematisch 9-18 gevonden waarden. Zowel in de experimenten als in de simulaties vinden we dat di-tertbutyl silaan een enigzings grotere preferentie heeft voor de warme kant in vergelijking met het meer symmetrische tetra ethyl silaan. De gemeten themoforetische beweging voor deze drie niet-polaire mengsels voldoet aan de gebruikelijke trends, waar de komponent met de grotere molaire massa, kleinere diameter en de grotere Hildebrandt parameter accumuleren aan de koudere kant. Hoofdstuk 3 bevat een systematische study van binaire mengsels van niet-bolvormige n-alkaan molekulen op basis van twee verschillende methoden, een konvectieve methode met een thermogravitatie kolom (TK) en de niet-konvectieve TDRV techniek. In het algemeen wordt een goede overeenkomst tussen de twee methoden gevonden. Des al niet te min zijn er enige discrepanties met recent gepubliceerde TK-methode data [85]. Zowel de TK als de TDRV methode geven niet de toename in de thermische diffusie koefficient voor hogere alkanen te zien zoals eerder waargenomen. We menen dat de reden hiervoor ligt in de relatief grote cel dikte in de TK methode [85] in vergelijk met die gebruikt in de huidige experimenten. Een grotere cel dikte leidt tot een mindere nauwkeurigheid in de bepaling van de massa scheiding bij de twee einden van de TK. Dit is in het bijzonder van belang voor mengsels van dekaan met hogere alkanen. Onze metingen laten zien dat de zware komponent zich naar de koude kant beweegt, precies zoals in mengsels van bol vormige molekulen.

De hoofdstukken 4 en 5 beschrijven onze studie van de invloed van de keten lengte en de mate van vertakking op het thermodiffusieve gedrag van lineare en vertakte alkanen in benzeen (een schijfvormig molekuul) m.b.v. de TDRV techniek, rooster model berekeningen als mede ONEMD simulaties. In hoofdstuk 4 wordt gevonden dat de experimenteel bepaalde Soret koefficienten in alkaan/benzeen mengsels afhangen van de moleculaire massa en struktuur van het alkaan, en in het bijzonder van de temperatuur en samenstelling van het mengsel. De Soret koefficient $S_{\mathrm{T}}$ van de n-alkanen zijn negatief voor deze mengsels met benzeen, wat betekent dat de alkanen zich naar de warme kant bewegen. De grootte van de Soret koefficient neemt af met toenemende keten lengte en toenemende alkaan koncentratie. De temperatuur afhankelijk van de Soret koefficient hangt af van de kompositie van het mengsel en de alkaan keten lengte; de helling van $S_{\mathrm{T}}$ versus de temperatuur veranderd 
van positief naar negatief met toenemende keten lengte bij ongeveer gelijke kompositie van de beide molekulen. Om de invloed van de vorm van de molekulen op de Soret koefficient te bestuderen, zijn eveneens experimenten en berekeningen aan mengsels van gebranchte alkanen in benzeen gedaan. Onze resultaten laten zien dat een toenemende vertakking leidt tot sterkere preferentie van de alkanen voor de warme kant. Het vertakkings effect is zo sterk dat voor 2,2,4-tri methyl pentaan/benzeen mengsels de Soret koefficient van voorteken veranderd voor hoge koncentraties alkaan, terwijl voor gelijke molaire koncentraties in 2,2,3-tri methyl butaan/benzeen mengsels de Soret koefficient altijd positief is binnen het onderzochte temperatuur bereik. Een rooster model dat is ontwikkeld door Luettmer Strathmann [90], reproduceerd de experimentele data voor de lineaire alkanen. Voor de vertakte alkanen is de interpretatie van de data gekompliceerder. Een vergelijk van de experimentele data voor vertakte heptaan isomeren met die voor de lineaire ketens voor heptaan en heptadekaan laat zien dat het effect van vertakking groter is dan dat van het moleculaire gewicht. Dit is niet wat men verwacht op basis van de themodynamische eigenschappen van de pure alkanen. Voor de vertakte alkanen is de relatieve verandering van de Soret koefficient evenredig met de verandering van het inertiaal moment alsgevolg van het toenemende aantal methyl groepen. In hoofdstuk 5 worden simulaties voor de massa-diffusie en Soret koefficienten besproken, teneinde een beter begrip te verkrijgen voor het effect van de vorm van een molekuul op voor mengsels van heptaan en benzeen. Deze mengsels zijn bestudeerd voor verschillen koncentraties terwijl het effect van vertakking is onderzocht voor gelijke molekulaire koncentraties van vertakte heptanen (3-MH, 2,3-DMP en 2,4-DMP) in benzeen. De gesimuleerde massadiffusie en Soret koefficienten hebben dezelfde trend als experimenteel wordt waargenomen. De gesimuleerde waarden voor $S_{\mathrm{T}}$ waren echter systematisch ongeveer $3 \times 10^{-3} \mathrm{~K}^{-1}$ lager dan de experimentele waarden. De gevonden afname van de grootte van $S_{\mathrm{T}}$ voor equi-molaire heptaan/benzeen mengsles kan niet verklaard worden door het effect van massa en molekuul grootte. Het effect van de vorm van molekulen, welke de vloeistof struktuur en de kinetische eigenschappen van het mengsel beinvloedt, speelt kennelijk een belangrijke rol.

Hoofdstuk 6 is een systematische studie van het thermo diffusieve gedrag van associerende molekulen in binaire mengsels van water, gedeutereerd water, dimethyl sulfoxide (DMSO), methanol, ethanol, aceton, 1-propanol, 2-propanol en propionaldehyde. Verandering van het voorteken van $S_{\mathrm{T}}$ wordt gevonden bij hoge water koncentraties van $x_{w}^{ \pm}=0.8-0.92$. Voor 
veel systemen kan deze verandering van voorteken gerelateerd worden aan strukturele veranderingen van tetrahedrale orde naar keten-achtige orde. Een karakteristieke eigenschap van de onderzochte systemen is dat de Soret koefficient bij een bepaalde water koncentratie $x_{w}^{\times}$ onafhankelijk wordt van de temperatuur. Terwijl voor niet-associatieve mengsels $S_{\mathrm{T}}\left(x_{w}^{\times}\right) \neq 0$, geldt voor de associatieve mengsels dat $S_{\mathrm{T}}\left(x_{w}^{\times}\right)=0$. Voor die systemen waarvoor $x_{w}^{ \pm}=x_{w}^{\times}$ wordt gevonden dat $x_{w}^{ \pm}$afhangt van de verhouding van de verdampings enthalpien van de pure komponenten. Voor deze systemen bestaat het hydrophobe deel uit twee koolstof atomen. Voor waterige mengsels van methanol en propanolen, korreleert de voorteken verandering met de grootte van het hydrophobe deel. We hebben tevens twee polaire, niet-waterige systemen onderzocht: ethanol/DMSO en ethanol/DMSO. Het systeem methanol/DMSO vertoont geen voorteken verandering noch een intersectie punt van de isothermen, hetgeen wellicht samenhangt met de gelijkenis tussen methanol en DSMO. Ethanol/DMSO mengsels gedragen zich zoals typische associatieve mengsels met $x_{w}^{ \pm} \neq x_{w}^{\times}$.

In het laatste hoofdstuk 7 vergelijken we de TDRV techniek met de TL methode. Drie verschillende systemen zijn onderzocht, met Soret koefficienten varieerend van $10^{-3}$ tot $10^{-1} \mathrm{~K}^{-1}$. Goede overeenstemming voor de met de twee technieken gemeten Soret koefficienten wordt gevonden voor het systeem met de grotere Soret koefficient. De limiet van de TL methode wordt bereikt voor de kleinere Soret koefficienten $10^{-3} \mathrm{~K}^{-1}$, zodat voor het daarmee gepaard gaande lage optische kontrast geen betrouwbare metingen kunnen worden gedaan. De typische langzaam diffunderende molekulen zoals polymeren en kolloiden hebben een relatief hoge Soret koefficient $10^{-1} \mathrm{~K}^{-1}$, hetgeen de TL methode geschikt maakt voor deze systemen. Een andere limitering van de TL methode is dat de karakteristieke equilibratie tijd veel kleiner dient te zijn dan de konvectie tijd. Onderzoek van grote kolloiden met de TL methode is daarom niet mogelijk. Het grote voordeel van de TL techniek is dat deze redelijk snel is en dat de kosten voor de bouw van een TL opstelling relatief laag zijn in vergelijking met een TDRV opstelling. Een belangrijke voorwaarde voor de laser straal is dat deze een perfect Gausisch profiel heeft. De koherentie lengte kan echter vrij klein zijn. In het geval van eventuele toekomstige benchmarking experimenten die ook de TL methode omvatten, dient men systemen met zorg te kiezen opdat de signaal-ruis verhouding groot genoeg is om nauwkeurige TL metingen te kunnen doen. Het is eveneens redelijk eenvoudig om een andere golflengte te gebruiken, zodat het toevoegen van een kleurstof vermeden kan worden 
door gebruik te maken van de natuurlijke absorptie van de reeds aanwezige molekulen. Het is gebleken dat ionische vloeistoffen goede kandidaten zijn om de TL methode te valideren, welke systemen ook voor de meeste andere technieken bruikbaar zijn. 


\section{Acknowledgement}

First of all, I would like to sincerely appreciate my promoters: Prof. Jan K. G. Dhont and Prof. Wim J. Briels and my co - promoter Dr. Simone Wiegand. They offer me the opportunity to pursue my Ph.D. study in the group of Soft Condensed Matter. I have got a perfect working conditions: nice working atmosphere, modern equipment, the possibilities to participate in different courses, and student seminars.

Secondly, I would like to thank all the people in our group, who support me during my work. I appreciate a lot of active suggestions from Hartmut Kriegs about the technical side of my work. Dear Hui, thanks a lot for your cooperation during the TDFRS measurements. I would like also to thank my collages and friends, Christoph Gögelein, Marie Göcking, Peter Holmqvist, Hans Hoffmann, Dzina Kleshchanok, Kyongok Kang, Pavlik Lettinga, Gerhard Meier, Mathieu McPhie, Gerhard Nägele, Karin Sellinghoff, Wiebke Sager, Dieter Triefenbach, Sylvia de Waal and Zhenkun Zhang.

Finally, I would like to thank my collaborators Pablo Blanco together with Prof. Mounir Bou-Ali from Mondragon University in Spain, Prof. Jutta Luettmer-Strathmann from the University of Akron in the United States and Prof. Rio Kita at Tokai University in Japan for many fruitful discussions and constructive ideas. Without the cooperation with Prof. Florian Müller-Plathe, the head of Theoretical Physical Chemistry group in Darmstadt University, the simulation work would not be possible. The members of his group Mohammad Alaghemandi, Elena Algaer, Thomas Müller, Eddie Rossinsky, Mei-mei Zhang and Konstantin Tarmyshov are appreciated for their cooperation. 


\section{Curriculum Vitae}

Pavel Polyakov was born on May 13th, 1982 in Novosibirsk city, Russian Federation. He studied physics at Novosibirsk State University, Russian Federation, where he received his Bachelor degree in 2003. In 2003, he started his Master study with Dr. A.A. Paletsky and Prof. O.P. Korobeinichev in the Institute of Chemical Kinetics and Combustion SB RAS on the subject of Studying the structure of the flame of energy materials on the basis of nitramines and active binders, and two year later he was received a Master degree in chemical physics. At the end of 2005, he joined the soft matter group in IFF, Forschungszentrum Jülich, Germany. There, he started his Ph.D studies under the supervision of Dr. Simone Wiegand and Prof. Jan K. G. Dhont. His Ph.D work focuses on the Study of thermal diffusion behavior of simple binary mixtures. 


\section{Publications}

- Polyakov, P.; Müller-Plathe, F. and Wiegand, S.: Reverse nonequilibrium molecular dynamics calculation of the Soret coefficient in liquid heptane/benzene mixtures, $J$. Phys. Chem. B, in print.

- Polyakov, P. and Wiegand, S.: Comparison between thermal lens technique and thermal diffusion forced Rayleigh scattering method, Phys. Chem. Chem. Phys., in print.

- Blanco, P; Polyakov, P.; Bou-Ali, M. and Wiegand, S.: Thermal diffusion and molecular diffusion values for some alkane mixtures: A comparison between thermogravitational column and thermal diffusion forced Rayleigh scattering, J. Phys. Chem. B, 112, 83408345, 2008.

- Polyakov, P.; and Wiegand, S.: Systematic study of the thermal diffusion in associated mixtures, J. Chem. Phys., 128, 034505, 2008.

- Polyakov, P.; and Wiegand, S.: Study of the thermal diffusion behavior of simple and associated mixtures, $8^{\text {th }}$ International Meeting on Thermodiffusion, Bonn, June 9-13, 2008, Germany.

- Kita, R.; Polyakov, P. and Wiegand, S.: Ludwig-Soret effect of poly(N-isopropylacrylamide) temperature dependence study in monohydric alcohols, Macromolecules., 40, 1638-1642, 2007.

- Polyakov, P.; Zhang, M; Müller-Plathe, F. and Wiegand, S.: Thermal diffusion measurements and simulations of binary mixtures of spherical molecules, J. Chem. Phys., 127, 014502, 2007.

- Polyakov, P.; Luettmer-Strathmann, J. and Wiegand, S.: Study of the thermal diffusion behavior of alkane/benzene mixtures by thermal diffusion forced Rayleigh scattering experiments and lattice model calculations, J. Phys. Chem. B, 110, 26215-26224, 2006. 
- Polyakov, P.; Wiegand, S.: Experimental study of the thermal diffusion behaviour of mixtures consisting of simple and chain like molecules using thermal diffusion forced Rayleigh Scattering, Thermodiffusion : Basics and Applications / ed.: M. M. Bou-Ali, J. K. Platten. - Arrasate-Mondragon, Mondragon University, 2006. - S. 399 - 407

- Polyakov, P.; Korobeinichev, O.; Paletsky, A.; Tereschenko, A. and Volkov E.: Investigation of RDX chemical structure at 1 atm using Molecular Beam Mass-Spectrometry and modeling, The Proceedings European Combustion Meeting, CD version, paper 79, Louvain-la-Neuve, Belgique, 2005.

- Paletsky, A.; Korobeinichev, O.; Tereschenko, A.; Volkov, E. and Polyakov, P.: Flame structure of HMX/GAP propellant at high pressure, The Proceedings of the Combustion Institute (Editors: Barlow; R.; Colket, M.; Chen, J. and Yetter, R.), 30: 2105-2112, 2005. 


\section{Bibliography}

[1] The molar mass, the density and the boiling point for the chemical provided by the company Aldrich. See http://www.sigmaaldrich.com.

[2] The density for carbon tetrabromide we found at https://www.wpi.edu/Academics/Depts/Chemistry/Courses/General/flotation.html and http://lrc-srvr.mps.ohio-state.edu/under/chemed/qbank/7/7-3.que.

[3] private communication: J.A. Madariaga and C. Santamaría, University Bilbao, University of Basque Country.

[4] Nist Chemistry WebBook. NIST Standard Reference Database 69, http://webbook.nist.gov/chemistry/, June 2005.

[5] Chem3D, Ver. 10. CambridgeSoft, Cambridge, MA, 2006.

[6] S. Alves, A. Bourdon, and A. M. F. Neto. Generalization of the thermal lens model formalism to account for thermodiffusion in a single-beam Z-scan experiment: determination of the Soret coefficient. J. Opt. Soc. Am. B, 20:713-718, 2003.

[7] S. Alves, G. Demouchy, A. Bee, D. Talbot, A. Bourdon, and A. M. F. Neto. Investigation of the sign of the Soret coefficient in different ionic and surfacted magnetic colloids using forced Rayleigh scattering and single-beam Z-scan techniques. Philos. Mag., 83:2059-2066, 2003.

[8] J. S. Anderson and K. Saddington. The use of radioactive isotopes in the study of the diffusion of ions in solution. J. Chem. Soc., 5:381-386, 1949.

[9] P-.A. Artola and B. Rousseau. Microscopic interpretation of a pure chemical contribution to the Soret effect. J. Phys. Rev. Lett., 98:125901, 2007.

[10] A. M. Awwad, S. F. Al-Azzawi, and M. A. Salman. Volumes and viscosities of benzene+alkane mixtures. Fluid Phase Equilib., 31:171-182, 1986. 
[11] M.G.G. Bagnoli. Modeling the Thermal Diffusion Coeffcients. Diplom, Technical University of Denmark, 2004.

[12] A. F. M. Barton, editor. CRC Handbook of Solubility Parameters and Other Cohesion Parameters, volume 2nd ed. CRC Press Inc., Boca Laton, FL, 1991.

[13] R.J. Bearman, J.G. Kirkwood, and M. Fixman. Statistical-mechanical theory of transport processes. 10. the heat transport in binary liquid solutions. advances in chemical physics. J. Adv. in Chem. Phys., 1:1-13, 1958.

[14] A. Becker, W. Köhler, and B. Müller. A scanning Michelson interferometer for the measurement of the concentration and temperature derivative of the refractive- index of liquids. Berichte der Bunsen-gesellschaft-physical chemistry chemical physics, 99:600-608, 1995.

[15] V. P. Belousov and M. Yu. Panov. Thermodynamic properties of aqueous solutions of organic substances. CRC Press, Boca Raton, FL, 1994.

[16] H. J. C. Berendsen, J. P. M. Postma, W. F. van Gunsteren, A. Di Nola, and J. R. Haak. Molecular dynamics with coupling to an external bath. J. Chem. Phys., 81:3684, 1984.

[17] J.A. Bierlein. A phenomenological theory of the Soret diffusion. J. Chem. Phys., $23: 10,1955$.

[18] P. Blanco, M.M. Bou-Ali, J.K. Platten, J.A. Madariaga, P. Urteaga, and C. Santamaría. Thermodiffusion coefficient for binary liquid hydrocarbon mixtures. J. Non-Equilib. Thermodyn., 32:309-317, 2007.

[19] V. Boersma-Klein and A.E. De Vries. The influence of the distribution of atomic masses within the molecule on thermal diffusion. J. Physica, 32:717-733, 1966.

[20] M. M. Bou-Ali, O. Ecenarro, J. A. Madariaga, C. M. Santamaria, and J. J. Valencia. Thermogravitational measurement of the Soret coefficient of liquid mixtures. J. Phys.: Condens. Matter, 10:3321-3331, 1998.

[21] L. Brambilla and G. Zerbi. Local order in liquid $n$-alkanes: evidence from Raman spectroscropic study. Macromolecules, 38:3327-3333, 2005. 
[22] M.S. Burberry, J. A. Morrel, A.C. Albrecht, and R.L. Swofford. Local mode overtone intensities of $\mathrm{C}-\mathrm{H}$ streching modes in alkanes and methyl substituted benzenes. $J$. Chem. Phys., 70:5522, 1979.

[23] I. Cacelli, G. Cinacchi, G. Prampolini, and A. Tani. Modeling benzene with singlesite potentials from ab initio calculations: a step toward hybrid models of complex molecules. J. Chem. Phys., 120:3648-3656, 2004.

[24] D.R Caldwell and S.A. Eide. Separation of seawater by Soret diffusion. J. Deep-See Research, 8:965, 1985.

[25] A.C. Carter and J.M. Harris. Comparison of models describing the thermal lens effect. J. Appl. Opt., 23:476, 1984.

[26] J. Chan, J. J. Popov, S. Kolisnek-Kehl, and D. G. Leaist. Soret coefficients for aqueous polyethylene glycol solutions and some tests of the segmental model of polymer thermal diffusion. J. Solution Chem., 32:197-214, 2003.

[27] J. Chang and S. I. Sandler. Interatomic Lennard-Jones potentials of linear and branched alkanes calibrated by Gibbs ensemble simulations for vapor-liquid equilibria. J. Chem. Phys., 121:7474-7483, 2004.

[28] J.S. Chickos and W.E. Acree. Enthalpies of vaporization of organic and organometallic compounds. J. Phys. Chem. Ref. Data, 32:519, 2003.

[29] K. Clusius and G Dickel. Zur Trennung der Chlorisotope. Naturwissenschaften, 27:148-149, 1939.

[30] P. Costeseque, D. Fargue, and P. Jamet. Thermodiffusion in porous media and its consequences. In W. Köhler and S. Wiegand, editors, Thermal nonequilibrium phenomena in fluid mixtures, Lecture Notes Physics, pages 389-427. Springer, Berlin, 2000.

[31] R.T Cygan and C.R. Carrigan. Time-dependent Soret transport: Applications to brine and magma. J. Chem. Geology, 95:201-212, 1992.

[32] T. E. Daubert and R. P. Danner. Physical and Thermodynamic Properties of Pure Chemicals: Data Compilation. Hemisphere, Washington, 1989-1992. 
[33] B-J. de Gans, R. Kita, B. Müller, and S. Wiegand. Negative thermodiffusion of polymers and colloids in solvent mixtures. J. Chem. Phys., 118:8073-8081, 2003.

[34] B-J. de Gans, R. Kita, S. Wiegand, and J. Luettmer Strathmann. Unusual thermal diffusion in polymer solutions. Phys. Rev. Lett., 91:245501, 2003.

[35] S. R. de Groot and P. Mazur. Non-equilibrium Thermodynamics. Dover, New York, 1984.

[36] C. Debuschewitz and W. Köhler. Molecular origin of thermal diffusion in benzene plus cyclohexane mixtures. Phys. Rev. Lett., 87:055901(1-4).

[37] J. Demichowicz-Pigoniowa, M. Mitchell, and H.J.V. Tyrrell. Thermal diffusion in the $n$-heptane-benzene system at $25^{\circ}$ C. J. Chem Soc. A - Inorg. phys. Theor., 2:307-309, 1971.

[38] Y. Demirel and S.I. Sandler. Effects of concentration and temperature on the coupled heat and mass transport in liquid mixtures. Int. J. of Heat and Mass Transfer, 45:7586, 2002.

[39] K.G. Denbigh. The heat transport in binary regular solutions. J. Trans Faraday Soc, 48:1, 1952.

[40] V.M. Dorogush and G.D. Rabinovich. Use of the thermodiffusional columns with packing for determining the Soret coefficient of the mixture of benzene and carbon tetrachloride. Inzh.-Phys. Zh., 41:503, 1981.

[41] E.L. Dougherty and H.G. Drickamer. A theory of thermal diffusion in liquids. J. Chem. Phys., 23:295-309, 1955.

[42] E.L. Dougherty and H.G. Drickamer. Thermal diffusion and molecular motion in liquids. J. Chem. Phys., 59:443-449, 1955.

[43] S. Duhr, S. Arduini, and D. Braun. Thermophoresis of DNA determined by microfluidic fluorescence. Eur. Phys. J. E, 15:277-286, 2004.

[44] S. Duhr and D. Braun. Thermophoretic depletion follows Boltzmann distribution. Phys. Rev. Lett., 96:168301(1-4), 2005. 
[45] J. F. Dutrieux, J. K. Platten, G. Chavepeyer, and M. M. Bou-Ali. On the measurement of positive Soret coefficients. J. Phys. Chem. B, 106:6104-6114, 2002.

[46] O. Ecenarro, J. A. Madariaga, J. Navarro, C. M. Santamaria, J. A. Carrion, and J. M. Saviron. Fickian and thermal-diffusion coefficients from liquid thermogravitational columns. J. Phys.: Condens. Matter, 2:2289-2296, 1990.

[47] J. T. Edward. Molecular volumes and Stokes-Einstein equation. Journal of Chemical Education, 47:261, 1970.

[48] A. H. Emery and H. G. Drickamer. Thermal diffusion in polymer solutions. J. Chem. Phys., 23:2252-2257, 1955.

[49] H. Ertl and F.A.L. Dullein. Self-diffusion and viscosity of some liquids as a function of temperature. J. AIChE, 19:1215, 1973.

[50] S. Fayolle, T. Bickel, S. L. Boiteux, and A. Würger. Thermal diffusion of charged micelles. Phys. Rev. Lett., 95:208301(1-4), 2005.

[51] L. E. Ficke, H. Rodrigez, and J. Brennecke. J. Chem. Eng. Data, 2008.

[52] A. Firoozabadi, K. Ghorayeb, and K. Shukla. Theoretical model of thermal diffusion factors in multicomponent mixtures. AIChE Journal, 46:892-900, 2000.

[53] E. W. Fischer, G. R. Strobl, M. Dettenmaier, M. Stamm, and N. Steidle. Molecular orientational correlations and local order in normal-alkane liquids. Faraday Discuss. Chem. Soc., 68:26-45, 1979.

[54] D. Frenkel and B. Smit. Understanding Molecular Simulations. Academic Press, San Diego, 2002.

[55] G. Galliero, B. Duguay, J. P. Caltagirone, and F. Montel. On thermal diffusion in binary and ternary Lennard-Jones mixtures by non-equilibrium molecular dynamics. Philos. Mag., 83:2097-2108, 2003.

[56] G. Galliero, B. Duguay, J. P. Caltagirone, and F. Montel. Thermal diffusion sensitivity to the molecular parameters of a binary equimolar mixture, a non-equilibrium molecular dynamics approach. Fluid Phase Equilibr., 208:171-188, 2003. 
[57] K. Ghorayeb and A. Firoozabadi. Modeling multicomponent diffusion and convection in porous media. SPE Journal, 5:158 - 171, 2000.

[58] K. Ghorayeb, A. Firoozabadi, and T. Anraku. Interpretation of the unusual fluid distribution in the Yufutsu gas-condensate field. SPE Journal, 8:114 - 123, 2003.

[59] M. Giglio and Vendrami.A. Thermal lens effect in a binary-liquid mixture - new effect. Appl. Phys. Lett., 25:555-557, 1974.

[60] M. Giglio and A. Vendramini. Thermal-diffusion measurements near a consolute critical-point. Phys. Rev. Lett., 34:561-564, 1975.

[61] M. G. Gonzalez-Bagnoli, A. A. Shapiro, and E. H. Stenby. Evaluation of the thermodynamic models for the thermal diffusion factor. Philos. Mag., 83:2171-2183, 2003.

[62] R.C. Gordon, R.C.C Leite, R.C. Moore, S.P.S. Porto, and J.R. Whinnery. J. Appl. Phys., 36:3, 1965.

[63] K. E. Grew and T. L. Ibbs. Thermal Diffusion in Gases. University Press, Cambridge, 1952.

[64] B. Hafskjold. Computer simulations of thermal diffusion in binary fluid mixtures. In W. Köhler and S. Wiegand, editors, Thermal nonequilibrium phenomena in fluid mixtures, Lecture Notes in Physics, pages 3-23, Heidelberg, 2002. Springer.

[65] B. Hafskjold, T. Ikeshoji, and S. K. Ratkje. On the molecular mechanism of thermaldiffusion in liquids. Mol. Phys., 80:1389-1412, 1993.

[66] H. C. Helgeson. Some thermodynamic aspects of geochemistry. Pure \& Appl. Chem., $57: 31-44,1885$.

[67] S. Iacopini, R. Rusconi, and R. Piazza. The "macromolecular tourist": Universal temperature dependence of thermal diffusion in aqueous colloidal suspensions. Eur. Phys. J. E, 19:59-67, 2006.

[68] C.G. Jiang, M.Z. Saghir, S. Derawi, and M. Kawaji. Accuracy of the theoretical calculation of the Soret coefficient for water ethanol mixtures in comparison with experimental data. J. Non-Equilib. Thermodyn., 30:337-358, 2005. 
[69] J.C. Johnson and A.L. Beyerlein. Thermal diffusion in mixtures with associated reactions. J. Phys. Chem., 82:1430, 1978.

[70] L. J. T. M. Kempers. A comprehensive theory of the Soret effect in a multicomponent mixture. In W. Köhler and S. Wiegand, editors, Thermal nonequilibrium phenomena in fluid mixtures, volume LNP 584, pages 74-92. Springer, Berlin, 2002.

[71] L.J.T.M. Kempers. A thermodynamic theory of the Soret effect in a multicomponent liquid. J. Chem. Phys., 90(11):6541, 1989.

[72] R. Kita, G. Kircher, and S. Wiegand. Thermally induced sign change of Soret coefficient for dilute and semidilute solutions of poly(N-isopropylacrylamide) in ethanol. $J$. Chem. Phys., 121:9140-9145, 2004.

[73] R. Kita, P. Polyakov, and S. Wiegand. Ludwig-soret effect of poly(Nisopropylacrylamide): temperature dependence study in monohydric alcohols. Macromolecules, 40:1638-1642, 2007.

[74] R. Kita, S. Wiegand, and J. Luettmer Strathmann. Sign change of the Soret coefficient of poly(ethylene oxide) in water/ethanol mixtures observed by TDFRS. J. Chem. Phys., 121:3874-3885, 2004.

[75] W. Köhler and B. Müller. Soret and mass diffusion-coefficients of toluene $n$-hexane mixtures. J. Chem. Phys., 103:4367-4370, 1995.

[76] W. Köhler and R. Schäfer. Polymer analysis by thermal-diffusion forced Rayleigh scattering. In M. Schmidt, editor, New Developments in Polymer Analytics II, volume 151, pages 1-59. Springer, Berlin, 2000.

[77] W. Köhler and S. Wiegand, editors. Thermal nonequilibrium phenomena in fluid mixtures. Lecture Note in Physics. Springer, Berlin, 1 edition, 2002.

[78] P. Kolodner, H. Williams, and C. Moe. Optical measurement of the Soret coefficient of ethanol water solutions. J. Chem. Phys., 88:6512-6524, 1988.

[79] D. Kondepudi and Prigogine I. Modern Thermodynamics. John Wiley and Sons, New York, 1998. 
[80] H. Korsching. Soret coefficients of mixtures from normal alkanes and cyclic hydrocarbons. Z. Naturf. a, 24:444, 1969.

[81] H. Korsching. Thermal-diffusion factor of $n$-heptane-benzene. Z. Naturf. a, 29:1914, 1974.

[82] S. Kouris and C. Panayiotou. Dynamic viscosity of mixtures of benzene, ethanol and heptane at 298.15K. J. Chem. Eng. Data, 34:200-203, 1989.

[83] C. Lacito, A.A. Messias, D.N. amd Andrade, S.M. Lima, M.L. Baesso, and Catunda T. Thermal lens and Z-scan measurements: Thermal and optical properties of laser glasses-a Rewiew. J. Non - Cryst. Sol., 352:3582, 2006.

[84] S. M. Lambert, Y. Song, and J. M. Prausnitz. Equations of state for polymer systems. In J. V. Sengers, R. F. Kayser, C. J. Peters, and H. J. White Jr., editors, Equations of state for fluids and fluid mixtures, chapter 14, pages 523-588. Elsevier, Amsterdam, 2000.

[85] A. Leahy-Dios and A. Firoozabadi. Molecular and thermal diffusion coefficients of alkane-alkane and alkane-aromatic binary mixtures: Effect of shape and size of molecules. J. Phys. Chem. B, 111:191-198, 2007.

[86] W. B. Li, P. N. Segre, R. W. Gammon, J. V. Sengers, and M. Lamvik. Determination of the temperature and concentration-dependence of the refractive-index of a liquidmixture. J. Chem. Phys., 101:5058-5069, 1994.

[87] J.L. Lin, W.L. Taylor, W.M. Rutherford, and J. Millat. Measurement of the Transport Properties of Fluids. Blackwell Scientific, Oxford, 1991.

[88] G.R. Long and S. E. Bialkowski. Pulsed infrared laser thermal lens spectrophotometric determination of trace-level gas-phase analytes: quantitation of parts per billion dichlorodifluoromethane. J. Anal. Chem., 56:2806, 1984.

[89] C. Ludwig. Diffusion zwischen ungleich erwärmten orten gleich zusammengesetzter lösungen. Sitz. Ber. Akad. Wiss. Wien Math.-naturw. Kl, 20:539, 1856. 
[90] J. Luettmer-Strathmann. Two-chamber lattice model for thermodiffusion in polymer solutions. J. Chem. Phys., 119:2892, 2003.

[91] J. Luettmer-Strathmann. Lattice model for thermodiffusion in polymer solutions. Int. J. Thermophys., 26:1693-1707, 2005.

[92] N-Y.R. Ma, D. Stanford, and A.L. Beyerlein. Thermal diffusion in carbon tetrachloride-methanol and carbon tetrachloride-ethanol mixtures. J. Phys. Chem., 87:5464-5467, 1983.

[93] G.M. Martin and J.I. Siepmann. Transferable potentials for phase equilibria. 1. unitedatom description of $n$-alkanes. J. Phys. Chem. B, 102:2569-2577, 1998.

[94] E. P. C. Mes, W. Th. Kok, and R. Tijssen. Prediction of polymer thermal diffusion coefficients from polymer-solvent interaction parameters: Comparison with thermal field flow fractionation and thermal diffusion forced Rayleigh scattering experiments. International Journal of Polymer Anal. Charact., 8:133-153, 2003.

[95] G. Meyerhoff and K. Nachtigall. Diffusion, thermodiffusion, and thermal diffusion of polystyrene in solution. J. Polym. Sci., 57:227-239, 1962.

[96] G. Milano and F. Müller-Plathe. Cyclohexane-benzene mixtures: Thermodynamics and structure from atomistic simulations. J. Phys. Chem. B, 108:7415, 2004.

[97] K. Mizuno, S. Imafuji, T. Ochi, and S. Maeda. Hydration of the CH groups dimethyl sulfoxide probed by NMR and IR. J. Phys. Chem. B, 104:11001-11005, 2000.

[98] K. Mizuno, T. Ochi, and Y. Shindo. Hydrophobic hydration of acetone probed by nuclear magnetic resonance and infrared: Evidence for the interaction $\mathrm{CH} . . . \mathrm{OH}_{2} . J$. Chem. Phys., 109:9502-9507, 1998.

[99] J. Moreau and V. Loriette. Confocal dual-beam thermal-lens microscope: Model and experimental results. J. Jap. Appl. Phys., 45:7141, 2006.

[100] K. I. Morozov. Thermodiffusion in magnetic colloids. J. Magn. Magn. Mater., 201:248-251, 1999. 
[101] F. Müller-Plathe. Yasp: A molecular simulation package. Comput. Phys. Commun., $78: 77,1993$.

[102] K. Nachtigall and G. Meyerhoff. Die Messung der Diffusionskoeffizienten von Hochpolymeren in Lösung mit einer konvektionsfreien Thermodiffusionszelle. Makromolekulare Chemie, 33:85-88, 1959.

[103] S.K. Nath and R. Khare. New forcefield parameters for branched hydrocarbons. J. Chem. Phys., 115:10837, 2001.

[104] C. Nieto-Draghi, J. B. Ávalos, and B. Rousseau. Computing the Soret coefficient in aqueous mixtures using boundary driven nonequilibrium molecular dynamics. $J$. Chem. Phys., 122:114503, 2005.

[105] H. Ning. Thermal Diffusion Behavior of Complex Fluid Mixtures. PhD thesis, University of Twente, 2007.

[106] H. Ning, J. Buitenhuis, J. K. G. Dhont, and S. Wiegand. Thermal diffusion behavior of hard sphere suspensions. J. Chem. Phys., 125:204911, 2006.

[107] H. Ning, S. Datta, T. Sottmann, and S. Wiegand. Soret effect of nonionic surfactants in water studied by different transient grating setups. J. Phys. Chem. B, 2008. im press.

[108] H. Ning, J. K. G. Dhont, and S. Wiegand. Thermal-diffusive behavior of a dilute solution of charged colloids. Langmuir, 24:2426-2432, 2008.

[109] H. Ning, R. Kita, H. Kriegs, J. Luettmer-Strathmann, and S. Wiegand. Thermal diffusion behavior of nonionic surfactants in water. J. Phys. Chem. B., 110:10746-10756, 2006.

[110] H. Ning, R. Kita, and S. Wiegand. Soret effect in a nonionic surfactant system. Progr. Colloid Polym. Sci., 133:111-115, 2006.

[111] H. Ning and S. Wiegand. Experimental investigation of the Soret effect in acetone/water and water/dimethylsulfoxide mixtures. J. Chem. Phys., 125:221102, 2006.

[112] S.W. Norman and N.J. Dovichi. J. Appl. Phys., 67:1170, 1989. 
[113] A. Perronace, C. Leppla, F. Leroy, B. Rousseau, and S. Wiegand. Soret and mass diffusion measurements and molecular dynamics simulations of $n$-pentane- $n$-decane mixtures. J. Chem. Phys., 116:3718-3729, 2002.

[114] R. Piazza. Thermal diffusion in ionic micellar solutions. Philos. Mag., 83:2067-2085, 2003.

[115] R. Piazza and A. Guarino. Soret effect in interacting micellar solutions. Phys. Rev. Lett., 88:208302, 2002.

[116] J. K. Platten, M. M. Bou-Ali, P. Costeseque, J. F. Dutrieux, W. Köhler, C. Leppla, S. Wiegand, and G. Wittko. Benchmark values for the Soret, thermal diffusion and diffusion coefficients of three binary organic liquid mixtures. Philos. Mag., 83:1965$1971,2003$.

[117] J. K. Platten, M. M. Bou-Ali, and J. F. Dutrieux. Precise determination of the Soret, thermodiffusion and isothermal diffusion coefficients of binary mixtures of dodecane, isobutylbenzene and 1,2,3,4-tetrahydronaphthalene (contribution of the University of Mons to the benchmark test). Philos. Mag., 83:2001-2010, 2003.

[118] J.K. Platten, M.M. Bou-Ali, and J.F. Dutrieux. Enhanced molecular separation in inclined thermogravitational columns. J. Phys. Chem. B, B 2003:11763-11767, 2003.

[119] A. Polt, B. Platzer, and G. Maurer. Parameter der thermischen zustandsgleichung von bender für 14 mehratomige reine stoffe. Chem. Tech. (Leipzig), 22:216-224, 1992.

[120] R.C. Powell. Physics of Solid-State Laser Materials. Springer, New York, 1998.

[121] S. A. Putnam and D. G. Cahill. Transport of nanoscale latex spheres in a temperature gradient. Langmuir, 21:5317-5323, 2005.

[122] G.D. Rabinovich. Separation of Isotopes and other Mixtures by Thermal Diffusion. Atomizdat, Moscow, 1981.

[123] J. Rauch and W. Köhler. Diffusion and thermal diffusion of semidilute to concentrated solutions of polystyrene in toluene in the vicinity of the glass transition. Phys. Rev. Lett., 88:185901, 2002. 
[124] J. Rauch and W. Köhler. On the molar mass dependence of the thermal diffusion coefficient of polymer solutions. Macromolecules, 38:3571-3573, 2005.

[125] D. Reith. Thermal diffusion in binary Lennard-Jones liquids. PhD thesis, Universität Mainz und Max-Planck-Institut für Polymerforschung, 1998.

[126] D. Reith and F. Müller-Plathe. On the nature of thermal diffusion in binary LennardJones liquids. J. Chem. Phys., 112:2436-2443, 2000.

[127] R. Rey, L.C. Pardo, E. Llanta, K. Ando, D.O. Lopez, J.Li. Tamarit, and M. Barrio. $\mathrm{X}$-ray and molecular dynamics study of liquid structure in pure methylchloromethane compounds. J. Chem. Phys., 112:7505, 2000.

[128] D. E. Rosner, R. S. Israel, and B. La Mantia. "Heavy" species Ludwig-Soret transport effects in air-breathing combustion. Combustion and Flame, 123:547-560, 2000.

[129] P. Rossmanith and W. Köhler. Polymer polydispersity analysis by thermal diffusion forced Rayleigh scattering. Macromolecules, 29:3203-3211, 1996.

[130] B. Rousseau, C. Nieto-Draghi, and J. Bonet Avalos. The role of molecular interaction in the change of sign of the Soret coefficient. Europhys. Lett., 67:976-982, 2004.

[131] R.L. Rowley, S.C. Yi, D.V. Gubler, and J.M. Stoker. Mutual diffusivity, thermal conductivity, and heat of transport in binary liquid mixtures of alkanes in chloroform. $J$. Chem. Ing. Data, 33:362-366, 1988.

[132] R.L. Rowley, S.C. Yi, and J.M. Stoker. Mutual diffusivity, thermal conductivity, and heat of transport in binary liquid mixtures of alkanes in carbon tetrachloride. J. Fluid Phase Equilibria, 36(2):219-233, 1987.

[133] J. S. Rowlinson and F. L. Swinton. Liquids and liquid mixtures. Butterworths, London, 3rd edition, 1982.

[134] K. Rubini, R. Francesconi, A. Bigi, and F. Comelly. Excess molar enthalpies and heat capacities of dimethyl sulfoxide + seven normal alkanols at $303.15 \mathrm{~K}$ and atmospheric pressure. Thermochmica Acta, 452:124, 2007. 
[135] R. G. Rubio, C. Menduiña, and M. Diaz Peña. Order effects in the excess thermodynamic properties of benzene + alkane mixtures. J. Chem. Soc., Faraday Trans. 1, 80:1425-1434, 1984.

[136] P. Rudolph, T. Boeck, and P Schmit. Thermodiffusion and morphological stability in convectionless crystal growth systems from melts and melt-solutions. J. Cryst. Res. Technol., 31:221-229, 1996.

[137] R. Rusconi, L. Isa, and R. Piazza. Thermal-lensing measurement of particle thermophoresis in aqueous dispersions. J. Opt. Soc. Am. B, 21:605-616, 2004.

[138] W.M. Rutherford. Calculation of thermal diffusion factors for the methane- $n$-butane system in the critical and liquid regions. J. AIChE, 9:841, 1963.

[139] W.M. Rutherford. Effect of mass distribution on the isotopic thermal diffusion of substituted benzenes. J. Chem. Phys., 81:6136, 1984.

[140] W.M. Rutherford. Effect of mass distribution on the isotopic thermal diffusion of benzene. J. Chem. Phys., 86:5217, 1987.

[141] W.M. Rutherford and H.G. Drickamer. Theory of thermal diffusion in liquids and the use of pressure to investigate the theory. J. Chem. Phys., 22:1157, 1954.

[142] M. P. Santos, S. L. Gomez, Bringuier E., and Neto A. M. F. Thermodiffusion in a multicomponent lyotropic mixture in the vicinity of the critical micellar concentration by using the Z-scan technique. Phys. Rev. E., 77:011403, 2008.

[143] T. Sato and R. Buchner. The cooperative dynamics of the H-bond system in 2-propanol water mixtures: Steric hindrance effects of non-polar head group. J. Chem. Phys., 119:10789, 2003.

[144] R.L. Saxton, E.L. Dougherty, and H.G. Drickamer. Thermal diffusion in binary liquid mixtures of simple symmetry. J. Chem. Phys., 122:1166, 1954.

[145] M. E. Schimpf and J. C. Giddings. Characterization of thermal-diffusion in polymersolutions by thermal field-flow fractionation - effects of molecular-weight and branching. Macromolecules, 20:1561-1563, 1987. 
[146] M. E. Schimpf and S. N. Semenov. Mechanism of polymer thermophoresis in nonaqueous solvents. J. Phys. Chem. B, 104:9935-9942, 2000.

[147] J. Schirdewahn, A. Klemm, and L. Waldmann. Thermodiffusion in D2-HT und anderen Wasserstoffgemischen. Z. Naturforsch., 16a:133-144, 1961.

[148] J. W. Schroer and P. A. Monson. Phase behavior in a quadrupolar hard sphere interaction site model of benzene. J. Chem. Phys., 114:4124-4130, 2001.

[149] P. N. Segre, R. W. Gammon, and J. V. Sengers. Light-scattering measurements of nonequilibrium fluctuations in a liquid-mixture. Phys. Rev. E, 47:1026-1034, 1993.

[150] S. N. Semenov. Mechanism of particle thermophoresis in pure solvents. Philos. Mag., 83:2199-2208, 2003.

[151] S. N. Semenov and M. E. Schimpf. Molecular thermodiffusion (thermophoresis) in liquid mixtures. Phys. Rev. E, 72:041202(1-9), 2005.

[152] S.J. Sheldon, L.V. Knight, and Thorne J.M. Laser-induced thermal lens effect: a new theoretical model. J. Appl. Opt., 21:1663, 1982.

[153] J.J.C. Shieh. Thermal diffusion and segmental motion in binary $n$-alkane systems. J. Phys. Chem., 73:1508-1513, 1969.

[154] D. N. Shin, J. W. Wijnen, J.B.F.N. Engberts, and A. Wakisaka. On the origin of microheterogeneity: A mass spectrometric study of dimethyl sulfoxide-water binary mixture. J. Chem. Phys. B, 105:6759, 2001.

[155] K. Shukla and A. Firoozabadi. A new model of thermal diffusion coefficients in binary hydrocarbon mixtures. J. Colloid Interface Sci., 37:3331-3342, 1998.

[156] J. M. Simon, D. K. Dysthe, A. H. Fuchs, and B. Rousseau. Thermal diffusion in alkane binary mixtures - a molecular dynamics approach. Fluid Phase Equilibr., 151:151$159,1998$.

[157] C. Soret. Sur l'état d'équilibre que prend au point de vue de sa concentration une dissolution saaline primitivement homogène dont deux parties sont portées a des températures différentes. Arch. Sci. Phy. Nat. Lyenève, 3:48, 1879. 
[158] C. Soret. Influence de la température sur la distribution des sels dans leurs solutions. Acad. Sci. (Paris), 91:289-291, 1880.

[159] D.J. Stanford and A Beyerlein. End effects in thermogravitational thermal diffusion. thermal diffusion factors for the carbon tetrachloride-cyclohexane system. J. Chem. Phys., 58:4338, 1973.

[160] A. Striolo, C. McCabe, and P.T. Cummings. Organic-inorganic telechelic molecules: Solution properties from simulations. J. Chem. Phys., 125:104904, 2006.

[161] T. Takamuku, H. Maruyama, K. Watanabe, and T. Yamaguchi. Structure of 1propanolwater mixtures investigated by large-angle X-ray scattering technique. J. Solution Chem., 33:641, 2003.

[162] T. Takamuku, K. Saisho, S. Nozawa, and T. Yamaguchi. X-ray diffraction studies on methanol water, ethanol water, and 2 propanol water mixtures at low temperatures. $J$. Molec. Liq., 119:133-146, 2005.

[163] K. Thyagarajan and P. Lallemand. Determination of thermal-diffusion ratio in a binary mixture by forced Rayleigh-scattering. Opt. Commun., 26:54-57, 1978.

[164] L. J. Tichacek, W. S. Kmak, and H. G. Drickamer. Thermal diffusion in liquids - the effect of non-ideality and association. J. Phys. Chem., 60:660-665, 1956.

[165] D.J. Trevoy and H.G. Drickamer. Thermal diffusion in binary liquid hydrocarbon mixtures. J. Chem. Phys., 17:1120, 1949.

[166] F. Trouton. On molecular latent heat. J. Phil. Mag., 18:54, 1884.

[167] H. J. V. Tyrell. Diffusion and Heat Flow in Liquids. Butterworth, London, 1961.

[168] P. Urteaga, M.M. Bou-Ali, J.A. Madariaga, C. Santamaría, P. Blanco, and J.K. Platten. Thermodiffusion: Basics and applications. pages 449-458. Mondragon Unibertsitateko Zerbitzu Editoriala, 2006.

[169] S. Van Vaerenberg, J.C. Legros, and J.C. Dupin. First results of Soret coefficient measurement experiment. J. Adv. Space. Res., 16(8):69, 1995. 
[170] S. M. Vechi and M. S. Skaf. Molecular-dynamics simulations of dimethylsulfoxidemethanol mixtures. J. Chem. Phys., 123, 2005.

[171] T. Voelker, E. Blums, and S. Odenbach. Determination of the Soret coefficient of magnetic particles in a ferrofluid from the steady and unsteady part of the separation curve. Int. J. Heat Fluid Flow, 47:4315-4325, 2004.

[172] A. Voit. Untersuchung von Transportprozessen in binären Flüssigkeiten mit Hilfe thermischer Linsen. Diplom, University Bayreuth, 2003.

[173] L Waldmann. Handbuch der Physik ed S Flügge. Springer, Berlin, 1958.

[174] L. Waldmann. Transporterscheinungen in gasen von mittleren druck. In S. Flüegge, editor, Handbuch der Physik, volume 12, pages 295-514. Springer Verlag, Berlin, 1958 .

[175] D. Walker, S.E. DeLong, and J.C. Dupin. Soret separation of mid-ocean ridge basalt magma. J. Contrib. Mineral. Petrol., 79:231-240, 1982.

[176] T. R. Walsh. Towards an anisotropic bead-spring model for polymers: a Gay-Berne parametrization for benzene. Molecular Phys., 100:2867-2876, 2002.

[177] J.T. Wescott, P. Kung, and S.K. Nath. Vapour liquid coexistence properties and critical points of two branched alkanes series. Fluid Phase Equilibria, 208:123, 2003.

[178] F. C. Whitmore. Experimental study of thermal diffusion in dilute solutions of high polymers. J. Appl. Phys., 31:1858-1864, 1960.

[179] S. Wiegand. Thermal diffusion in liquid mixtures and polymer solutions. J. Phys.: Condens. Matter, 16:R357-R379, 2004.

[180] S. Wiegand, R. Kita, and H. Ning. Universal concentration dependence of the Soret coefficient in aqueous systems. J. Noneq. Thermod, 32:193-201, 2007.

[181] S. Wiegand and W. Köhler. Measurement of transport coefficients by an optical grating technique. In Thermal Nonequilibrium Phenomena in Fluid Mixtures, volume LNP584, pages 189-210. Springer, Berlin, 2002. 
[182] G. Wittko and W. Köhler. Precise determination of the Soret, thermal diffusion and mass diffusion coefficients of binary mixtures of dodecane, isobutylbenzene and 1,2,3,4-tetrahydronaphthalene by a holographic grating technique. Philos. Mag., 83:1973-1987, 2003.

[183] G. Wittko and W. Köhler. Universal isotope effect in thermal diffusion of mixtures containing cyclohexane and cyclohexane-d $\mathrm{d}_{12}$. J. Chem. Phys., 123:014506, 2005.

[184] G. Wittko and W. Köhler. Universal isotope effect in thermal diffusion of mixtures containing cyclohexane and cyclohexane-d $\mathrm{d}_{12}$. J. Chem. Phys., 123:014506, 2005.

[185] G. Wittko and W. Köhler. On the temperature dependence of thermal diffusion of liquid mixtures. EPL, 78:46007, 2007.

[186] C.L. Yaws. Chemical Properties Handbook. McGraw Hill, New York, 1999.

[187] C. Yokoyama, T. Takagi, and S. Takahashi. J. Int. Thermod., 11(3):477, 1989.

[188] K. J. Zhang, M. E. Briggs, R. W. Gammon, and J. V. Sengers. Optical measurement of the Soret coefficient and the diffusion coefficient of liquid mixtures. J. Chem. Phys., 104:6881-6892, 1996.

[189] M. Zhang and F. Müller-Plathe. Reverse nonequilibrium molecular-dynamics calculation of the Soret coefficient in liquid benzene/cyclohexane mixtures. J. Chem. Phys., 123:124502(1-8), 2005.

[190] M. M. Zhang and F. Müller-Plathe. The Soret effect in dilute polymer solutions: influence of chain length, chain stiffness and solvent quality. J. Chem. Phys., 125, 2006.

[191] X. S. Zhao, B. Chen, S. Karaborni, and J. I. Siepmann. Vapor-liquid and vapor-solid phase equilibria for united-atom benzene models near their triple points: The importance of quadrupolar interactions. J. Phys. Chem. B, 109:5368-5374, 2005.

[192] L.L Zheng, D.J. Larson, and H Zhang. Role of thermotransport (Soret effect) in macrosegregation during eutectic/off-eutectic directional solidification. J. Cryst. Grow., 191:243, 1998. 
